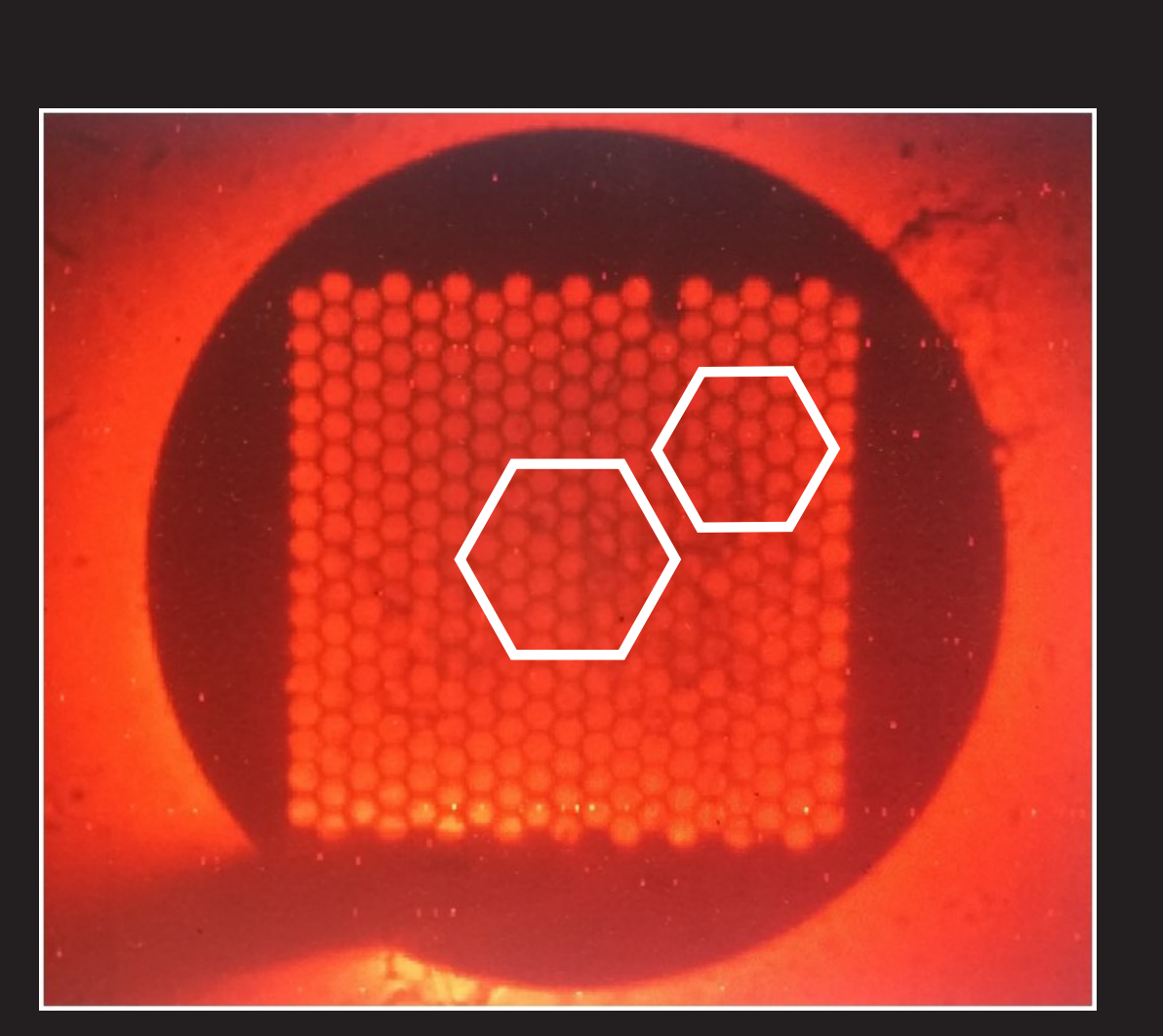

Towards electrostatic doping approaches in ultra-thin body semiconductor materials and devices

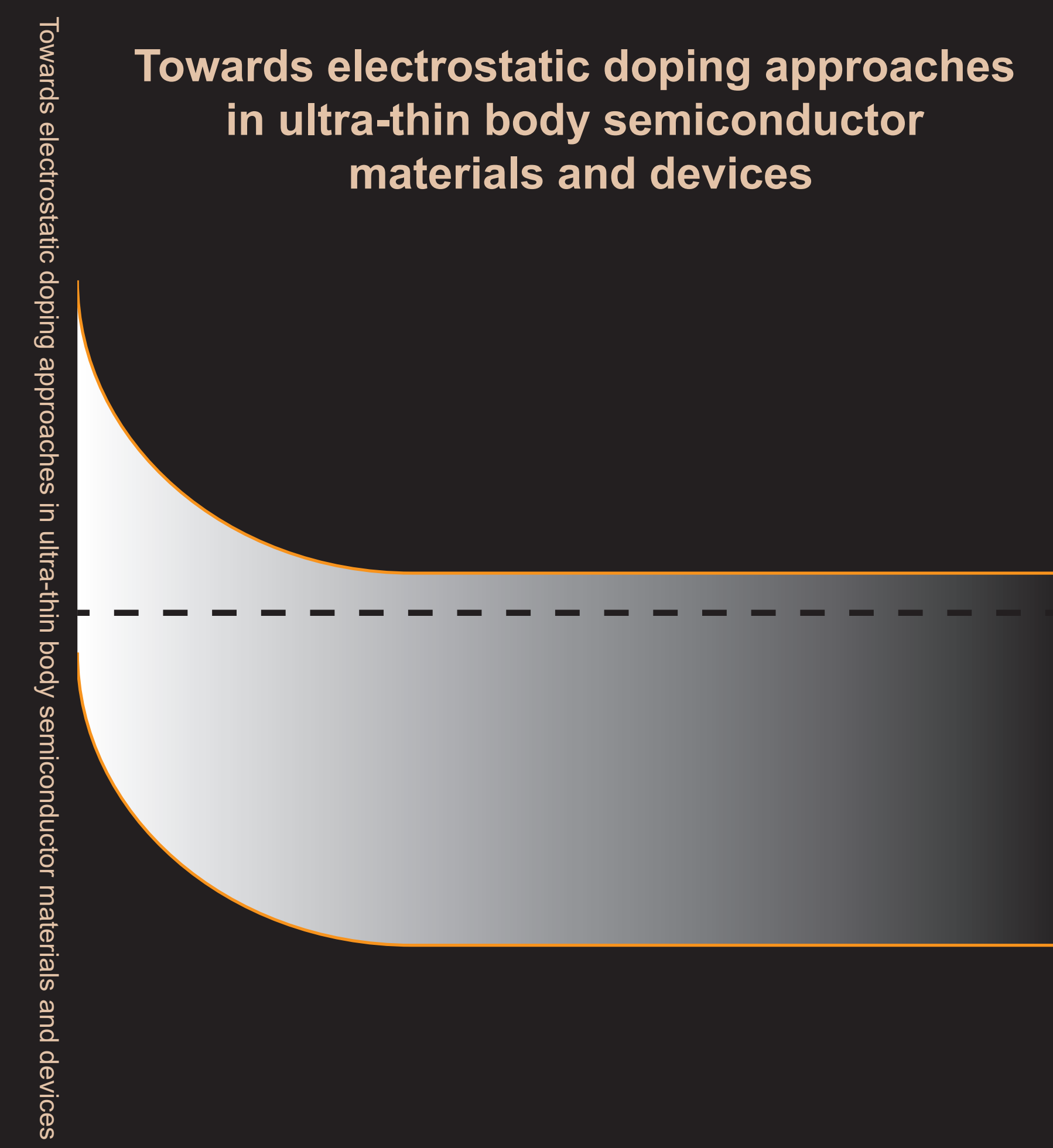

Gaurav Gupta 


\section{Towards electrostatic doping approaches in ultra-thin body semiconductor materials and devices}


Members of the dissertation committee:

$\begin{array}{rll}\text { prof. dr. } & \text { J.N. Kok } & \text { University of Twente (chairman and secretary) } \\ \text { prof. dr. } & \text { L.K. Nanver } & \text { University of Twente (supervisor) } \\ \text { prof. dr. } & \text { J. Schmitz } & \text { University of Twente (supervisor) } \\ \text { dr. ir. } & \text { R.J.E. Hueting } & \text { University of Twente (co-supervisor) } \\ \text { dr. } & \text { A.Y. Kovalgin } & \text { University of Twente } \\ \text { prof. dr. ir. } & \text { H.J.W. Zandvliet } & \text { University of Twente } \\ \text { dr. ir. } & \text { J. Klootwijk } & \text { Philips Research, Eindhoven } \\ \text { prof. dr. } & \text { S. Cristoloveanu } & \text { IMEP-LAHC, Minatec, Grenoble } \\ \text { prof. dr. } & \text { T. Suligoj } & \text { University of Zagreb, Croatia } \\ \text { prof. dr. } & \text { M.J. Kumar } & \text { Indian Institute of Technology (IIT), Delhi }\end{array}$

$\widehat{N \mathcal{W O}} \mid \begin{aligned} & \text { Applied and } \\ & \text { Engineering Sciences }\end{aligned}$

MESA+

INSTITUTE FOR NANOTECHNOLOGY

This work is part of the project "Towards polycrystalline GaN/AlGaN devices in silicon technology" (no. 13145) and is financially supported by the Applied and Engineering Science division (TTW) of the Netherlands Organization for Scientific Research (NWO). This work has been carried out at the MESA+ Institute for Nanotechnology, University of Twente.

(C) 2020 by Gaurav Gupta, The Netherlands. All rights reserved. No parts of this thesis may be reproduced, stored in a retrieval system or transmitted in any form or by any means without permission of the author. Alle rechten voorbehouden. Niets uit deze uitgave mag worden vermenigvuldigd, in enige vorm of op enige wijze, zonder voorafgaande schriftelijke toestemming van de auteur.

Typeset with $\mathrm{HT}_{\mathrm{E}} \mathrm{X}$.

Cover design and layout: PhD candidate

Back cover image: Infrared electroluminescence micrograph of the $\mathrm{Pd} / \mathrm{MoO}_{x} / \mathrm{n}-\mathrm{Si}$ diode at a constant forward current drive.

Printed by: IPSKAMP Printing, Enschede, The Netherlands.

ISBN 978-90-365-5018-5

DOI $\quad 10.3990 / 1.9789036550185$

https:/ / doi.org/10.3990/1.9789036550185 


\title{
TOWARDS ELECTROSTATIC DOPING APPROACHES IN ULTRA-THIN BODY SEMICONDUCTOR MATERIALS AND DEVICES
}

\section{DISSERTATION}

\author{
to obtain \\ the degree of doctor at the University of Twente, \\ on the authority of the rector magnificus, \\ prof. dr. T.T.M. Palstra, \\ on account of the decision of the doctorate board \\ to be publicly defended \\ on Friday 12 June 2020 at 16:45 hrs
}

by

\section{Gaurav Gupta}

born on the $13^{\text {th }}$ of January 1986 in Kota, Rajasthan, India 
This dissertation has been approved by:

prof. dr. L.K. Nanver (supervisor)

prof. dr. J. Schmitz (supervisor)

dr. ir. R.J.E. Hueting (co-supervisor) 
In the loving memory of my father and to the three beautiful ladies in my life: my mother, wife and sister. 


\section{ABSTRACT}

In the last seven decades there has been a revolution in the field of microelectronics which started with the invention of the bipolar transistor in 1948 and led to present day advanced computers and handheld devices such as smart phones. Continuous advancements in silicon (Si) based semiconductor devices (such as diodes, bipolar and field effect transistors) with their ever shrinking dimensions have fueled this technology revolution so far. The ability to dope $\mathrm{Si}$ with chemical impurities such as arsenic (As) or phosphorous $(\mathrm{P})$ to control its conductivity played a key role in the remarkable success of the $\mathrm{Si}$ technology platform till date.

However, with the continuous downscaling of Si device dimensions to the nanometer regime and evolution of complex device architectures such as nanowires, nanofins and fully-depleted silicon-on-insulator (FD-SOI), it is increasingly difficult to match the device requirements by employing conventional chemical doping technologies. In addition, doping is even more challenging in emerging materials such as graphene, carbon nanotubes, 2-D materials, gallium nitride $(\mathrm{GaN})$ and other wide band gap semiconductors. These materials are increasingly being sought for new application areas such as internet of things (IoT), artificial intelligence (AI) and wearable electronics. Among these materials, $\mathrm{GaN}$ is particularly interesting for both electronic and optical device applications. However, not only its doping is challenging, its production is also expensive which currently limits its widespread commercial applications. Monolithic integration of functional GaN based devices on a state-of-the-art Si CMOS platform could open up new applications in the area of high-power devices, RF electronics, lighting, sensing and display technologies and that with a reduced cost.

This thesis broadly deals with two aspects. Firstly, it describes possible alternatives to chemical doping in dimensionally scaled semiconductor devices based on alternative emerging materials and investigates the socalled "electrostatic doping (ED)" approach as a possible solution. In this work, various reported ED approaches have been reviewed and new device concepts have been proposed and investigated via TCAD simulations along with electrical and optical measurements of experimentally realized devices. The conditions for ED, i.e. inducing localized n-type or p-type regions in semiconductor using suitable metal work functions and gate biases, have been established. Among various possible ED based devices, the thesis particularly focuses on exploring the prospects of utilizing high-barrier Schottky contacts for devices such as LEDs, bipolar-transistors without adding any chemical doping. The thesis also explores the use of extreme work function contacts such as molybdenum oxide $\left(\mathrm{MoO}_{\mathrm{x}}\right)$ for realizing 
high-barrier diodes with bipolar conduction capabilities.

Secondly, this thesis investigates the possibility of utilizing atomic layer deposition (ALD) grown polycrystalline (poly-)GaN thin-films for device applications as a potential solution to low-cost GaN-on-Si technology platform. In this direction, various electrical and optical properties of ALD poly-GaN thin-films have been studied and their potential applications are discussed. The thesis also examines the applicability of various ED ideas developed in this work for ultimately realizing the functional ED-based devices in poly-GaN thin films. 


\section{SAMENVATTING}

In de afgelopen zeventig jaar heeft er op het gebied van de micro-elektronica een revolutie plaatsgevonden, die geïnitieerd was door de uitvinding van de bipolaire transistor in 1948 en geleid heeft naar de geavanceerde computers en mobiele toepassingen. De aanhoudende vooruitgang in de realisatie van silicium ( $\mathrm{Si}$ ) gebaseerde halfgeleider componenten (zoals diodes, bipolaire en veldeffect transistoren), die gepaard ging met de constante afname van de afmetingen daarvan, was tot dusver de drijvende kracht voor deze technologische revolutie. De mogelijkheid om Si te doteren met chemische verontreinigingen zoals arsenicum (As) of fosfor $(\mathrm{P})$ om de geleidbaarheid te controleren, heeft ook een sleutelrol gespeeld in het opmerkelijke succes van de huidige $\mathrm{Si}$ technologie.

Echter, met de aanhoudende afname van de transistorafmetingen naar de nanometer regime en de toename van de complexiteit van transistorarchitecturen, zoals gerealiseerd in nanodraden, nanovinnen en volledig gedepleteerde silicium-op-isolatie (FD-SOI), wordt het steeds moeilijker om de traditionele chemische doteringstechnieken toe te passen. Bovendien is het doteren zelfs een grotere uitdaging in alternatieve materialen zoals grafeen, carbon nanobuisjes, 2-D materialen, gallium nitride (GaN) en andere brede "band gap" (verboden band) halfgeleiders. Deze materialen worden met toenemende mate ontwikkeld en onderzocht voor nieuwe toepassingen zoals internet van dingen (IoT), kunstmatige intelligentie en draagbare elektronica. Met name $\mathrm{GaN}$ is interessant, voor zowel elektronische als optische toepassingen. Het doteren van $\mathrm{GaN}$ is echter niet het enige probleem. De productie van $\mathrm{GaN}$ is relatief duur wat uiteindelijk het succes daarvan zou kunnen beperken. Daarom wordt er meer gekeken naar de mogelijkheden van monolitische integratie van functionele GaN gebaseerde componenten in een geavanceerd CMOS proces. Mocht dit succesvol zijn, dan zou dit kunnen leiden tot nieuwe toepassingen op het gebied van hoogvermogenscomponenten, RF elektronica, verlichting, sensoren en beeldschermtechnologieën, en dat met relatief lage kosten.

Dit proefschrift gaat in brede zin in op twee aspecten. Allereerst beschrijft het mogelijke oplossingen ter vervanging van chemische dotering in agressief geschaalde halfgeleider componenten die gerealiseerd zijn in alternatieve materialen, en onderzoekt het de zogeheten "elektrostatische dotering (ED)" methode als één van die mogelijke oplossingen. In dit onderzoekswerk is een recensie geschreven van verscheidende ED methodes die beschreven staan in de literatuur. Daarnaast zijn er nieuwe concepten van componenten bedacht die onderzocht zijn door middel van TCAD simulaties, en enkele componenten daarvan die gerealiseerd zijn, elek- 
trisch en optisch doorgemeten. De randvoorwaarden voor ED, d.i. het induceren van lokale n-type en p-type gebieden in een halfgeleider door middel van een geschikte uittreearbeid van metalen en gate instelspanningen, zijn vastgelegd. Tussen verscheidende mogelijke ED gebaseerde componenten, focusseert dit proefschrift zich vooral op het toepassen van hoge barrière Schottky contacten voor componenten zoals LEDs en bipolaire transistoren zonder daarbij chemische dotering toe te voegen. Het proefschrift bestudeert ook het gebruik van extreme uittreearbeid contacten zoals molybdeen oxide $\left(\mathrm{MoO}_{\mathrm{x}}\right)$ voor het realiseren van hoge barrière diodes met bipolaire geleidingsmogelijkheden.

Ten tweede, onderzoekt dit proefschrift de mogelijkheden om polykristallijne (poly-)GaN dunne films, die gegroeid zijn door middel van atomaire laag depositie (ALD), toe te passen in componenten als een potentiële oplossing voor goedkope GaN-op-Si technologie. Met dit uitgangspunt zijn er diverse elektrische en optische eigenschappen van ALD gegroeide poly-GaN dunne films bestudeerd en de potentiële toepassingen daarvan bediscussieerd. Dit proefschrift onderzoekt ook de toepasbaarheid van verscheidende ED ideeën die in dit werk bedacht zijn om uiteindelijk functionele ED-gebaseerde componenten in poly-GaN dunne films te realiseren. 


\section{CONTENTS}

\section{INTRODUCTION · 1}

1.1 Doping in semiconductor devices . 2

1.2 Polycrystalline GaN: material and devices . 3

1.3 ALD-grown poly-GaN thin films . 4

1.4 Challenges in poly-GaN device technology · 4

1.5 Role of electrostatic doping (ED) · 5

1.6 Thesis outline · 5

2 Electrostatic doping In SEMICONDUCtOR DEVICES * 9

2.1 Introduction $\cdot 10$

2.2 Electrostatic Doping Concept · 10

2.3 Electrostatic Doping Approaches: Discussion · 24

2.4 Conclusion $\cdot 28$

3 High-BARRIER Si-SCHOtTKy Diodes · 29

3.1 Introduction $\cdot 30$

3.2 Device Physics and TCAD Simulation · 32

3.3 Experimental Results · 42

3.4 Discussion · 48

3.5 Conclusion . 50

4 Bipolar CHARACTERISTICS OF HIGH-BARRIER Pd / $\mathrm{MOO}_{x} / \mathrm{N}-\mathrm{SI}$ DIODES · 51

$$
\begin{array}{rrr}
4.1 \quad \text { Introduction } & \cdot 52 \\
4.2 \text { High-barrier Schottky contacts } & \cdot 54 \\
4.3 \quad \text { Experimental procedure } & \cdot 55 \\
4.4 \quad \text { Material analysis } & \cdot 56 \\
4.5 \text { Diode I-V }-\mathrm{V} \text {-T) characteristics } & \cdot 57 \\
4.6 \quad \text { Sheet resistance measurements } & \cdot 59 \\
4.7 \quad \mathrm{C}-\mathrm{V} \text { measurements } & \cdot 60 \\
4.8 \text { Light-emission measurements } & \cdot 62 \\
4.9 \text { Surface barrier transistor (SBT) measurements } & \cdot 63 \\
4.10 \text { Discussion } & \cdot 68 \\
4.11 \text { Conclusion } & \cdot 70
\end{array}
$$

5 Electron-Hole Bilayer LED · 71 
5.1 Introduction $\cdot 72$

5.2 Theory and device operation · 72

5.3 Simulation parameters and models $\cdot 75$

5.4 Device operation: InAs EHB LED · 75

5.5 Efficiency estimation - 80

5.6 Device optimization: Body thickness · 81

5.7 Diode-mode operation $\cdot 83$

5.8 Si EHB LED · 84

5.9 Conclusion $\cdot 86$

6 Polycrystalline GaN/P-Si heterojunction diode $\cdot 87$

6.1 Introduction $\cdot 88$

6.2 Experimental details $\cdot 88$

6.3 Material characterization of the GaN layers · 89

6.4 Electrical Measurements · 90

6.5 Optical Measurements . 97

6.6 Discussion $\cdot 100$

6.7 Conclusions $\cdot 101$

7 ELECTRICAL PROPERTIES OF ALD-GROWN POLY-GAN THIN-FILMS $\cdot 103$

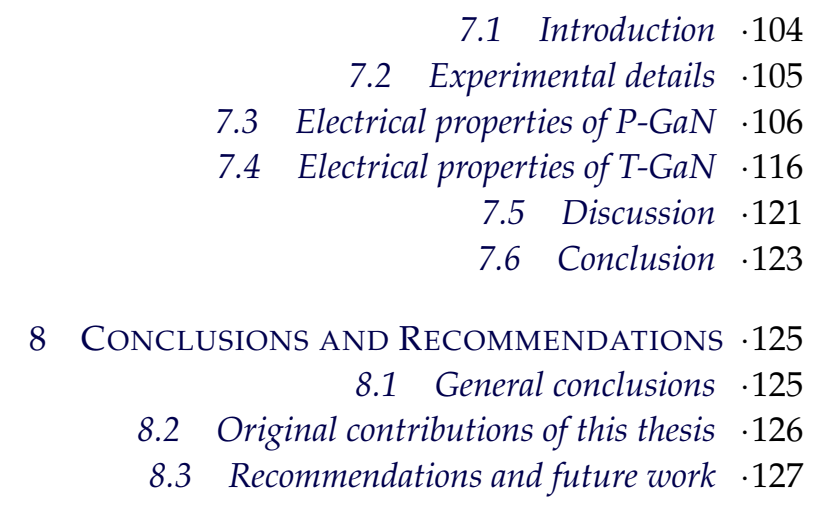

A Electric Field AND CARRIER CONCENTRATION IN THE ELECTRON-HOLE BILAYER SYSTEM $\cdot 129$

B SOlution of Poisson's EQuATION IN THE PRESENCE OF INVERSION LAYER $\cdot 131$

C DERIVATION OF CHARGE CARRIERS THERMAL GENERATION TIME $\cdot 135$

D INVERSION LAYER IN HIGH-BARRIER PD $/ \mathrm{MOO}_{x} / \mathrm{N}-\mathrm{SI}$ DIODES $\cdot 137$ 



\title{
INTRODUCTION
}

\begin{abstract}
In this chapter, the main motivation and objectives of this research are presented. The chapter starts with outlining the limitations of conventional chemical doping approaches in ultra-thin body semiconductor devices and alternative emerging materials other than silicon (Si). Then, a brief introduction to the potentially interesting polycrystalline gallium nitride $(\mathrm{GaN})$ material is given and possible device concepts based on this material are discussed, followed by the challenges involved in realizing such devices. Next the electrostatic doping approach is proposed as one potential solution to overcome the doping challenge in devices based on ultra-thin body semiconductors in general and polycrystalline GaN in particular. Finally, the outline of this thesis is presented.
\end{abstract}




\subsection{Doping in semiconductor devices}

Doping semiconductor materials by incorporating chemical impurities has been key to the development of today's cutting edge device technologies [1]. However with device dimensions of only a few nanometers, the conventional impurity doping faces challenges even in silicon (Si). The formation of junctions with extremely high doping gradients (a few $\mathrm{nm} /$ decade) is practically difficult $[2,3]$. At nanometer scale, a dopant concentration above its solid solubility limit would be required to achieve sufficiently low channel and contact resistances. In addition, random doping fluctuations and the resulting variability has been the key concern for manufacturing nano-scale devices and circuits with high yield [4-6].

The accurate control of doping type, level and spatial distribution in nanostructures such as a nanowire is also challenging because of their complex growth dynamics and geometrical constraints [7, 8]. For any nano-scale device, the requirement of a high carrier density along with unintentional and undesired ionized dopants in the active region points towards impurity-free doping solutions.

In case of alternative emerging material systems such as wide bandgap semiconductors (e.g. $\mathrm{GaN}, \mathrm{SiC}$ and $\mathrm{ZnO}$ ), it is still difficult to obtain either $\mathrm{p}$-type or $\mathrm{n}$-type regions via impurity doping. This is mainly because of deep donor or acceptor levels or dopant passivation via complex formation [9-12]. The chemical doping route is also not straightforward for many other semiconductor materials such as carbon nanotubes (CNTs) and emerging two-dimensional (2-D) materials (graphene, phosphorene, silicene and transition metal dichalcogenides (TMDs)) [13, 14]. For example, tunneling-field-effect-transistors (TFETs) based on ultra-thin channels and 2-D materials are potential contenders for beyond-CMOS (Complementary metal oxide semiconductor) technology as they promise sub-60 $\mathrm{mV} /$ decade sub-threshold slope [15]. However, their full potential has still not been realized experimentally. The reason for their limited performance lies in the difficulty in realizing highly doped junctions with a steep profile and low defect density, which is critical for an efficient tunneling process $[14,16]$.

Consequently, various approaches have been proposed in recent years to influence the electron and hole concentrations by means other than chemical doping. In many of these approaches, electrostatic interaction between the semiconductor and a different material at the interface governs the carrier density. These approaches are therefore referred to as "electrostatic doping (ED)"1 which will be discussed in more detail in the next chapter.

Among many alternative semiconductors discussed above, GaN, more specifically its thin-film polycrystalline counterpart grown on $\mathrm{Si}$, is potentially attractive technology platform for both electronic and optical

\footnotetext{
${ }^{1}$ The term "electrostatic doping" was originally introduced by Antonov and Johnson [17] in 1999 in relation to their CNT diode.
} 
applications and can benefit from ED approaches. Therefore a brief introduction to polycrystalline $\mathrm{GaN}$ is given below. A more detailed discussion on polycrystalline $\mathrm{GaN}$ will follow in the subsequent chapters of this thesis.

\subsection{Polycrystalline GaN: material and devices}

Mono-crystalline gallium nitride $(\mathrm{GaN})$ is currently a widely investigated compound semiconductor material for its interesting properties such as wide and direct bandgap, large breakdown field, suitability for high temperature and high frequency operation [18-20]. It is the material of choice for high (rf-) power transistors as well as optoelectronic devices such as LEDs. Despite this, its expensive production technology currently hinders its widespread commercial adaptability. Monocrystalline GaN is commonly deposited using epitaxial growth methods such as metal organic chemical vapor deposition (MOCVD) and molecular beam epitaxy (MBE) on sapphire substrate at temperatures ranging from $750^{\circ} \mathrm{C}$ to $1200^{\circ} \mathrm{C}$. The high production costs of $\mathrm{GaN}$ based devices can be brought down by employing inexpensive substrates such as silicon (Si). Other than the cost benefit, GaN-on-Si technology is also favorable for monolithic integration of GaN devices with state-of-the-art Si technology [21-23]. However, the requirement of a several-micrometer thick buffer layer [21, 24] for growing monocrystalline $\mathrm{GaN}$ on $\mathrm{Si}$ makes the idea of monolithic integration somewhat difficult and complex. In addition, the high temperature growth of monocrystalline $\mathrm{GaN}$ also limits its compatibility with several processes and applications where a low thermal budget is required.

In this regard, thin-film polycrystalline (poly-) $\mathrm{GaN}$, which has been largely unexploited so far, is an interesting alternative to its thick-film heteroepitaxial mono-crystalline counterpart for its advantages such as lower fabrication cost, choice of large area and flexible substrates and a lower thermal budget. In addition, the fact that GaN-based devices are exceptionally lucrative for many applications, particularly light emission, despite having comparatively high defect densities also raised the curiosity of some research groups to experiment with polycrystalline and even amorphous GaN films. Interestingly, Stumm and Drabold [25] in their early paper entitled "Can Amorphous GaN Serve as a Useful Electronic Material?" theoretically predicted that amorphous $\mathrm{GaN}$ is promising as a electronic material for device applications.

Poly-GaN thin-films deposited using reactive rf sputtering were reported as early as in 1972 [26, 27] by researchers at IBM corporation. Poly$\mathrm{GaN}$ thin-films have also been explored before for applications such as transparent TFTs [28, 29] ("transparent electronics"), LEDs [30] and field electron-emission [31-33] as illustrated in Fig. 1.1. 


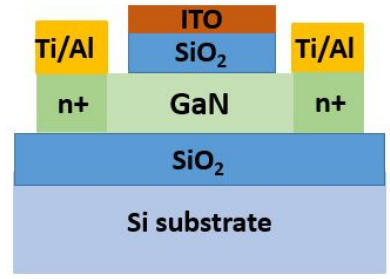

(a) Top gate GaN TFT

Chen et al., APL, 100, 2012

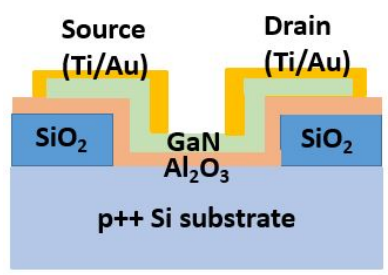

(c) Bottom gate Poly-GaN TFT Bolat et al., JVST-A, 33, 2015

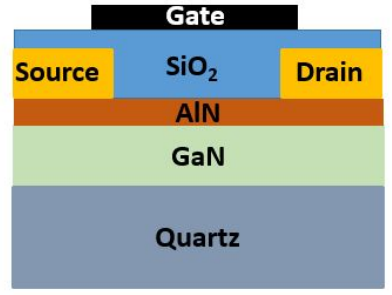

(b) Top gate AIN/GaN TFT

Chen et al., IEEE-EDL, 33, 2012

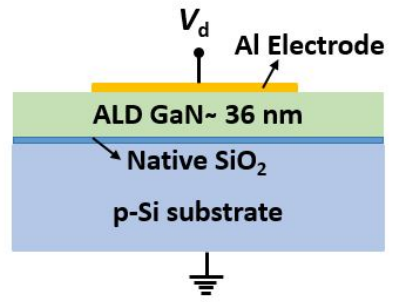

(d) Poly-GaN/Si diode Gupta et al., JAP, 124, 2018

Figure 1.1: Examples of various poly-GaN based devices reported till date.

\subsection{ALD-grown poly-GaN thin films}

Among several other reported techniques to deposit poly-GaN films such as rf-sputtering [26, 28], plasma-assisted molecular beam epitaxy (MBE) $[32,34]$ and pulse laser deposition (PLD) [35], atomic layer deposition (ALD) is an attractive solution [36, 37]. ALD features excellent waferlevel uniformity, conformal deposition and, most importantly, critical film thickness control. In addition, ALD offers the possibility to grow films at relatively low temperatures $\left(<500^{\circ} \mathrm{C}\right)[38,39]$. Several research groups have recently demonstrated devices such as poly-GaN thin-film transistors realized using ALD [39-41] with reasonable performance considering their polycrystalline nature.

\subsection{Challenges in poly-GaN device technology}

Generally, polycrystalline semiconductor devices do not perform up to par with their crystalline counterparts. For device applications in polycrystalline material several challenges should be taken into consideration $[28,35,42]$, such as obtaining large grain size and reducing the amount of grain boundaries and their role in charge transport, good device stability and reliability, obtaining low defect densities and improving the carrier mobility and lifetime, and good interface control with metals and dielectrics.

Both from technology and device functionality viewpoint, doping is also one important aspect that needs to be addressed for both electronic 
and optoelectonic device applications. Doping remains to be challenging in polycrystalline materials mainly because of the requirement of high temperature for dopant activation which forbids its use in films or processes where low temperature processing is important. Moreover, in-situ dopants mixing is not straightforward for certain deposition techniques such as ALD. The situation is even more complex in case of GaN where doping is anyways challenging in crystalline GaN particularly getting ptype material is difficult $[9,10]$. For ultra-thin poly-GaN films as deposited by ALD, forming a high device quality $p-n$ junction and their metal contacts becomes even more challenging. Even the polarization doping approach $[43,44]$, which is normally used in III-nitrides heterostructures to realize two-dimensional electron or hole gases (2DEGs, 2DHGs) is not an attractive solution for poly-GaN material due to their possibly small strain-induced piezoelectric polarization.

\subsection{Role of electrostatic doping (ED)}

One potential solution to doping problem in thin-film poly-GaN films for device applications could be the electrostatic doping (ED) [17, 45-48] approach where charge carriers are induced in the ultra-thin body semiconductor by using a suitable metal work function or by applying a gate bias. ED has already been demonstrated in various material systems and device geometries where conventional doping is otherwise challenging, as discussed in the next chapter. Moreover, the performance of ED-based devices are found to be at par with their chemically-doped counterparts.

Previously, the use of Schottky barrier (SB) contacts which is one possible type of ED was demonstrated to realize SB GaInN/GaN solar cell devices without any p-doped region [49], [50]. GaN based n-channel SBMOSFET has also been reported before [51]. Other possible forms of ED such as work function induced doping has also been utilized before to propose an n-type GaN MOSFET using TCAD simulations [52]. Even biasinduced p-type ED has been reported for a wide band gap material such as $\mathrm{ZnO}$ [53] though the source of holes is not clear from the report.

The formation of the inversion layer using high-barrier Schottky contacts [54-56] is also interesting for creating local n-type or p-type inverted regions. Such contacts could impart bipolar characteristics to the device which is attractive for applications such as light emission or could even be utilized to realize low-resistance ohmic contacts to the device. Therefore such ED approaches hold a lot of promise as a potential doping solution in ultra-thin poly-GaN devices and are the topic of discussion in this thesis.

\subsection{Thesis outline}

This thesis broadly deals with the subject of electrostatic doping as a potential solution to circumvent doping issues in ultra-thin body devices and 
wide bandgap materials such as GaN where conventional doping is otherwise challenging. In this work, various reported ED approaches have been investigated and new device concepts have been proposed via a theoretical study, TCAD simulations, device physics modeling as well as via electrical and optical measurements of experimentally realized devices. A significant part of thesis focuses on the physics of high-barrier Schottky diodes in Si and its applicability and adaptability in ultra-thin body devices. The use of extreme work function contacts such as $\mathrm{MoO}_{\mathrm{x}}$ has also been explored experimentally to realize work function induced ED. The other part of the thesis is dedicated to the study of electrical and optical properties of ALD-grown poly-GaN thin films and their potential applications. Most of the ED ideas explored in this thesis are initially conceptualized and experimented on $\mathrm{Si}$ while the properties of grown poly-GaN films have been investigated in parallel for their suitability for (ED-based) device applications. Specific research questions that this thesis will attempt to answer can be summarized as as follows:

- What are the various possible ED approaches and for which materials and device configurations are they most suited for?

- Is it possible to create local n-type or p-type layers in the semiconductors using extreme metal work functions or applied gate biasing? Can such approaches be utilized for innovative device applications such as light emitters or switches?

- Can high-barrier Schottky diodes be interesting for bipolar device applications? What are potential electrode materials with extreme work functions that can be used for realizing high-barrier diodes?

- What are the electrical properties of ALD-grown poly-GaN thin films and for what applications are they suitable?

- How feasible are the envisaged ED solutions in wide-band gap semiconductors, specifically poly-GaN? What are the challenges and limitations?

Below the outline for the subsequent chapters in this thesis is presented.

- Chapter 2: Electrostatic doping in semiconductor devices, based on work published in the IEEE Transactions on Electron Devices, 2017 [46]. This chapter reviews various reported ED approaches in Si and emerging semiconductor materials including their applicability to future CMOS devices.

- Chapter 3: High-barrier Si Schottky diodes, based on work published in the IEEE Transactions on Electron Devices, 2018 [56]. This chapter deals with the physics of high-barrier Schottky diodes via TCAD simulations and experimental investigation on Si substrates. 
- Chapter 4: Bipolar characteristics of high-barrier $\mathrm{Pd} / \mathrm{MoO}_{\mathrm{x}} / \mathrm{n}-\mathrm{Si}$ diodes. This chapter investigates the bipolar effects in high-barrier $\mathrm{Pd} / \mathrm{MoO}_{\mathrm{x}} / \mathrm{n}$-Si diodes via electrical and optical measurements.

- Chapter 5: The electron-hole bilayer LED, based on work published in Solid-State Electronics, 2020 [57]. This chapter describes a novel light emitting device concept based on the ED approach in an ultrathin-body semiconductor configuration using TCAD simulations.

- Chapter 6: The polycrystalline GaN/p-Si heterojunction diode, based on work published in the Journal of Applied Physics, 2018 [42]. This chapter investigates the charge carrier transport and electroluminescence properties of plasma-enhanced ALD deposited poly-GaN/p-Si diodes.

- Chapter 7: Electrical properties of ALD-grown poly-GaN thin films. This chapters describes and compares the electrical and optical properties of ultra-thin poly-GaN layers grown using plasma-enhanced ALD and thermal ALD processes.

- Chapter 8: Conclusions and Recommendations. This chapter summarizes the general conclusions of the aforementioned chapters and also provides recommendations and suggestions for future work in this direction. Lastly, a list of original key contributions of this thesis is provided. 



\title{
ELECTROSTATIC DOPING IN SEMICONDUCTOR DEVICES
}

\begin{abstract}
To overcome the limitations of chemical doping in nanometerscale semiconductor devices, electrostatic doping (ED) is emerging as a broadly investigated alternative to provide regions with a high electron or hole density in a semiconductor device. In this chapter, various reported ED approaches and related device architectures in different material systems are reviewed. The role of metal and semiconductor work functions, energy bandgap and applied electric field and the interplay between them for the induced ED is highlighted. The effect of interface traps on the induced charge is also addressed. In addition, the merits of electrostatically doped devices are underlined and the major roadblocks of these approaches for potential future CMOS technology are addressed.
\end{abstract}




\subsection{Introduction}

The limitations of conventional chemical doping approaches in ultra-thin body (UTB) devices and emerging semiconductor materials other than Si has been highlighted before in chapter 1 . In this direction, various electrostatic doping (ED) approaches has been reported in recent years where charge carriers are induced in UTB semiconductors using a suitable metal work function or applied local gate bias. These approaches include devices such as Schottky barrier (SB) metal-oxide-semiconductor fieldeffect transistors (MOSFETs) [58, 59], charge plasma based ultra-thin body (UTB) devices [60-63], reconfigurable FETs based on silicon (Si) nanowires [64-66], fully-depleted silicon-on-insulator (FD-SOI) devices [48, 67-69], FETs based on graphene [70-73], CNT [74-76] and 2-D materials [77, 78], electron-hole bilayer based TFETs [79-81] and light-emitting devices [82]. ED potentially offers ultra-sharp junctions with a well controlled carrier concentration profile and a reduced defect density. These features make $\mathrm{ED}$ an attractive alternative to conventional chemical doping for a broad range of electron devices.

This chapter presents an overview of various reported ED concepts, and is organized as follows. In section 2.2 the ED concept is first defined. Thereafter various reported ED concepts are reviewed under three proposed categories based on the nature of the underlying electrostatics. Here the focus will be on the electrostatics for different types of metal-semiconductor systems. Further, in section 2.3, the major challenges and possible drawbacks of the ED techniques are addressed. Section 2.4 summarizes our findings. For more detailed overview on ED based innovative FD-SOI devices, please refer to [48]. Further, for a more technology focused review on ED based TFETs and reconfigurable FETs refer to [47].

\subsection{Electrostatic Doping Concept}

Electrostatic doping (ED) is a technique in which charge carriers (electrons or holes) are induced in an ultra-thin semiconductor material as a result of its band alignment near its interface with another (semi)conducting material. In the ED approach, the relative separation between the Fermi level and the semiconductor energy bands, that governs the active doping concentration, is controlled by the potential and the work function of the electrode adjacent to the semiconductor body rather than by the chemical impurities as in conventional doping. The electrostatic condition at the metal-semiconductor (MS) interface ${ }^{1}$, which influences the band alignment, is a strong function of the metal work function $\left(\phi_{\mathrm{m}}\right)$, the semiconductor's energy bandgap $\left(E_{g}\right)$, electron affinity $\left(\chi_{\mathrm{s}}\right)$ and work function $\left(\phi_{\mathrm{s}}\right)$. In

\footnotetext{
${ }^{1}$ For simplicity sake, in this thesis ideal interfaces are considered unless stated otherwise. Parasitic charge, such as interface or a fixed charge, will also affect the electrostatics at interfaces.
} 
(a)

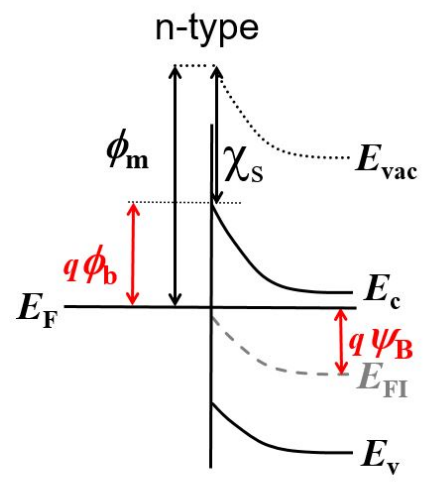

metal semiconductor (b)

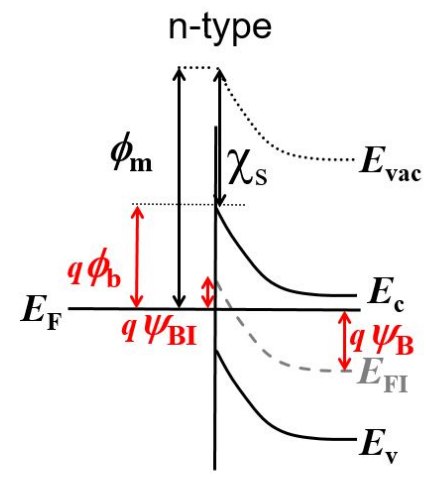

metal semiconductor

Figure 2.1: Schematic energy band diagrams for: (a) traditional n-type Schottky contact. A depletion layer is formed at the MS interface. (b) n-type Schottky contact with a large MS work function difference. The excessive band-bending near the interface results in inversion (p-type) charge in an n-type semiconductor. A similar situation can be obtained with Schottky contacts on a p-type semiconductor.

addition, the applied electric field, if any, also influences the electrostatic properties near the interface. Note that in this chapter, the focus is only on metal (or metallic compound) induced ED; other heterostructure based doping like polarization doping e.g. in III-nitrides [43] is beyond the scope of this work.

The ED approaches are subdivided into three categories: (1) Schottky barrier based devices, (2) work function induced ED, and (3) bias-induced ED. In the upcoming subsections, these ED techniques as applied to different devices and material systems will be reviewed.

\subsubsection{Schottky Barrier Based Devices}

Schottky barrier (SB) based devices are devices in which the current is limited by one or more Schottky contacts. The physics of a Schottky barrier formed at the metal-semiconductor (MS) interface has been well described earlier [83].

An SB with height $\mathrm{q} \phi_{\mathrm{b}}$ is formed when $\phi_{\mathrm{m}}>\phi_{\mathrm{s}}$ for an n-type semiconductor (Fig. 2.1(a)) and $\phi_{\mathrm{m}}<\phi_{\mathrm{s}}$ for a p-type semiconductor, where $\mathrm{q}$ is the elementary charge. The Schottky barrier height $(\mathrm{SBH}) q \phi_{\mathrm{b}}$ is $\left(\phi_{\mathrm{m}}-\chi_{\mathrm{s}}\right)$ for an n-type and $\left(E_{g}-\phi_{m}+\chi_{s}\right)$ for a p-type semiconductor. The presence of this potential barrier $\phi_{\mathrm{b}}$ at the MS interface results in the fundamental difference in the operation of SB devices from p-n junction devices. For the former, the MS interface, hence $\phi_{\mathrm{b}}$, fully controls the majority unipolar current [83]. The electron or hole emission in SB devices is governed by thermionic (-field) emission over (through) the barrier in contrast to p-n junction based devices, where processes such as drift-diffusion and 
band-to-band tunneling (BTBT) of both electrons and holes control the current.

However, for an ideal unipolar Schottky type operation, it is essential to limit the band bending such that for an n-type semiconductor, at the interface, the intrinsic Fermi-level $E_{F I}$ falls below the Fermi level $E_{F}$ which implies that $\phi_{b} \leqslant u_{T} \cdot \ln \left(N_{c} / n_{i}\right)$ where $N_{c}$ is the conduction band effective density of states, $n_{i}$ is the intrinsic carrier concentration, and $u_{T}$ is the thermal voltage $(=k T / q$, where $k$ is the Boltzmann constant and $T$ is the temperature). Therefore, the limiting conditions at the MS interface $(x=0)$ for a unipolar Schottky type operation can be expressed as:

$$
\left.\left(E_{\mathrm{c}}-E_{\mathrm{F}}\right)\right|_{x=0}=q \phi_{\mathrm{b}} \leqslant \mathrm{kT} \cdot \ln \left(\frac{\mathrm{N}_{\mathrm{c}}}{n_{\mathrm{i}}}\right)
$$

for an n-type semiconductor, and

$$
\left.\left(E_{F}-E_{v}\right)\right|_{x=0}=q \phi_{b} \leqslant k T \cdot \ln \left(\frac{N_{v}}{n_{i}}\right)
$$

for a p-type semiconductor. $E_{c}$ and $E_{v}$ represent the conduction band edge and valence band edge respectively and $N_{v}$ is the valence band density of states. The terms $k T \cdot \ln \left(\mathrm{N}_{\mathrm{c}} / \mathrm{n}_{\mathrm{i}}\right)$ and $k T \cdot \ln \left(\mathrm{N}_{\mathrm{v}} / \mathrm{n}_{\mathrm{i}}\right)$ are approximately equal to $E_{g} / 2$ for most semiconductors. Excessive band-bending at the MS interface may result in a bipolar type operation as discussed in the Section 2.2.2 and later investigated in more detail in chapter 3-4 of this thesis.

Next, some specific examples of SB devices which could be interesting for future CMOS, but also for electro-optical devices, are discussed.

\section{Schottky Barrier (SB) MOSFETs}

The idea to replace doped source/drains (S/Ds) in conventional MOSFETs with metal was first proposed by Nishi [84] in 1966. In 1968, Lepselter and Sze reported the first Si p-type MOSFET (PMOS) device where S/Ds were replaced by $\mathrm{PtSi}$ [58]. Thereafter a series of developments resulted in the metal S/Ds SB-MOSFET (Fig. 2.2(a)) technology which basically replaces impurity doped S/Ds in conventional MOSFETs with metal, typically silicide.

In this transistor the charge carriers are injected from the metal into the semiconductor channel via thermionic (-field) emission as in a Schottky diode. However, in the SB-MOSFET, the gate field further influences the effective barrier height and width as illustrated in Figs. 2.3 (a) and (b). The SB-MOSFET technology is claimed to offer several benefits for sub-30-nm scaling such as a low parasitic S/D resistance, sharp junctions, better control over the off-state leakage current, elimination of parasitic bipolar action, and low thermal budget processing [59]. Further, the use of mid-gap silicides allows the realization of SB-PMOS and SB-NMOS devices, as required for CMOS technology. Recently [86], the SB-FINFET was realized featuring a $6 \mathrm{mV} / \mathrm{dec}$ subthreshold swing at room temperature. 


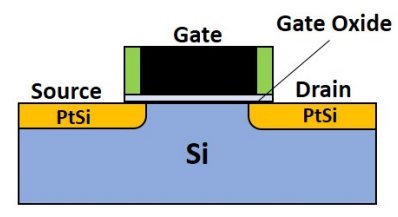

(a)

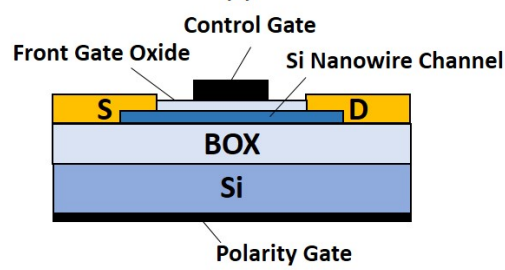

(c)

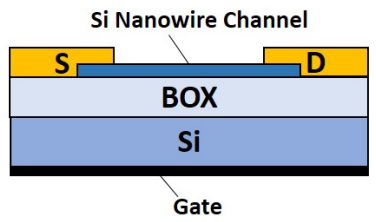

(b)

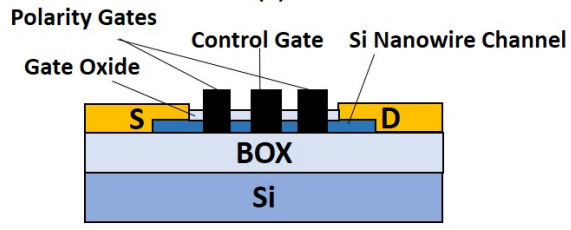

(d)

Figure 2.2: Schematic cross section of an (a) Si based SB-MOSFET [59], (b) Si nanowire based SB-MOSFET with single backgate design [85], Si nanowire based reconfigurable SB-MOSFET, (c) with front and back dual gate design [66], (d) with dual gate-all-around design [64].

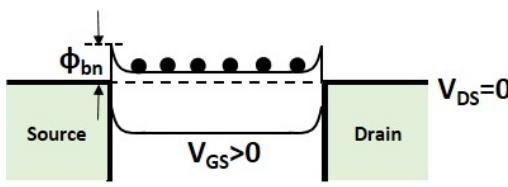

(a)

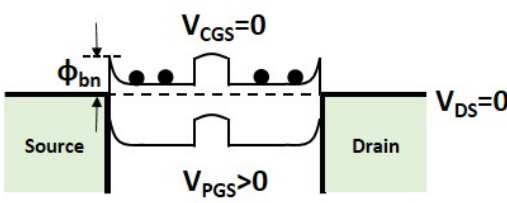

(c)

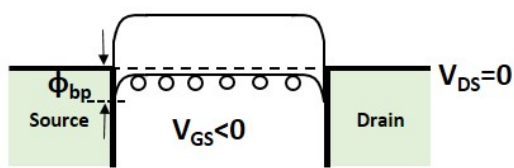

(b)

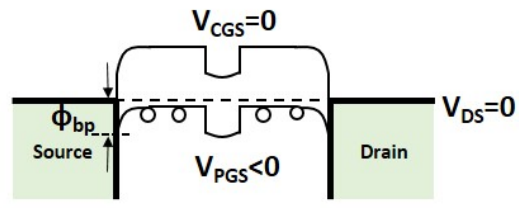

(d)

Figure 2.3: Schematic energy band diagram: Si based SB-MOSFET [59] (Fig 2.2(a)) for (a) n-type operation $\left(\mathrm{V}_{\mathrm{GS}}>0 \mathrm{~V}\right)$, (b) p-type operation $\left(\mathrm{V}_{\mathrm{GS}}<0 \mathrm{~V}\right)$. Si nanowire based reconfigurable SB-MOSFET [66] (Fig 2.2(c)) for (c) n-type operation ( $V_{\text {PGS }}>0$ $\mathrm{V})$, (d) p-type operation $\left(\mathrm{V}_{\mathrm{PGS}}<0 \mathrm{~V}\right)$. $\mathrm{V}_{\mathrm{PGS}}$ and $\mathrm{V}_{\mathrm{CGS}}$ denotes the polarity gate and control gate bias respectively. An electron (hole) channel is formed for a positive (negative) $\mathrm{V}_{(\mathrm{P}) \mathrm{GS}}$. The $\mathrm{V}_{\mathrm{CGS}}$ controls the current in the channel.

The concept of SB-MOSFETs has also been adopted in CNTs [75, 76, 87]. For a thorough review on SB-MOSFET technology refer to [59].

\section{Reconfigurable SB-MOSFETs}

The idea of using SB contacts for ED has also been embraced by the research community for applications other than traditional CMOS. The Si nanowire FET [85] with a SB source and drain were shown to exhibit ambipolar 


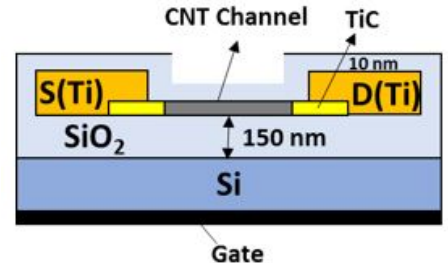

(a)

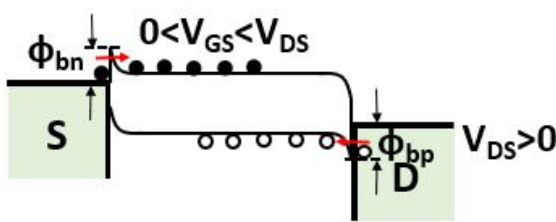

(b)

Figure 2.4: CNT SB light emitting FET, schematic (a) device cross section (b) energy band diagram. Electrons are injected in the CNT channel at the source electrode while holes are injected from the drain electrode into the channel [89].

characteristics (Fig. 2.2(b)). The ambipolarity in this device was successfully suppressed by the introduction of an additional terminal, i.e. polarity gate (Figs. 2.2(c) and (d)). This terminal offers a new degree of freedom in the device: the polarity of conduction can be controlled which was utilized in $[64-66,88]$ to demonstrate a reconfigurable FET operation. In these reconfigurable (or polarity controlled) devices, one gate electrode (control gate) controls the conduction through the channel while the other gate electrode (polarity gate) controls the polarity of conduction (see Figs. 2.3 (c) and (d)).

\section{SB electro-optical devices}

Misewich et al. [89] demonstrated polarized infrared light emission from a CNT SB-MOSFET structure (see Fig. 2.4(a)). The simultaneous injection of electrons and holes at the source and drain Schottky barrier junctions resulted in radiative recombination and thus light emission. The gate electrode was biased such that $\mathrm{V}_{\mathrm{GS}}>0$ but $\mathrm{V}_{\mathrm{GS}}<\mathrm{V}_{\mathrm{DS}}$. In this way band bending was formed in the opposite direction at source and drain electrodes which facilitated simultaneous injection of electrons and holes from those electrodes via narrow tunneling barriers, as shown in Fig. 2.4(b).

SB contacts have also been utilized to collect photo-generated carriers in solar cells [90]. SB based solar cells are interesting as they can be realized using only a single type ( $\mathrm{n}$ or $\mathrm{p}$ type) of chemical doping unlike conventional $\mathrm{p}$-i-n type cells which require both a p-type and n-type region to function. Other than in conventional Si-based material, the SB based solar cell has also been demonstrated in CNTs [91], cadmium sulphide (CdS) [92] and molybdenum disulfide ( $\left.\mathrm{MoS}_{2}\right)$ [93]. In addition, an SB based solar cell has been demonstrated in the GaInN/GaN material system without any p-type doped region [49], [50].

The choice of the electrode metal in these devices determines the resultant SBHs for electrons $\left(\phi_{\mathrm{bn}}\right)$ and for holes $\left(\phi_{\mathrm{bp}}\right)$ at the junction. Unipolar operation $\left(\phi_{\mathrm{bn}} \neq \phi_{\mathrm{bp}}\right)$ is desirable for conventional SB-FETs while ambipolar operation ( $\phi_{\mathrm{bn}} \approx \phi_{\mathrm{bp}}$ ) is interesting for polarity controlled SB-MOSFETs 
and light emitting devices [89]. Importantly, the current in the devices described so far is limited by the SBH.

\subsubsection{Work function induced ED}

Brattain and Bardeen [45] first reported an experimental observation that could be explained as metal induced doping. In their report, a thin layer of p-type conductivity is believed to be induced near the surface of the n-type bulk Ge via a Schottky point-contact which resulted in the bipolar-like amplification. Although noticed by only a few readers or authors, later on many reports actually followed this "scaled point-contact transistor" idea as discussed further in this section.

In this section the general analytical understanding of work function induced ED in one dimensional (1-D) Schottky contacts is first developed. Thereafter the case of two dimensional (2-D) gated Schottky contacts is discussed which is important for future CMOS devices such as ED-TFETs.

\section{1-D Schottky based devices}

Excessive band bending near the MS interface may result in charge carrier inversion (similar to gate-induced inversion in MOSFETs) as illustrated in Fig. 2.1(b). This will induce a bipolar type behavior in an otherwise unipolar 1-D Schottky diode which could be of interest to BTBT devices. As in a MOS capacitor [1], the onset of strong inversion near the interface is defined when the inversion charge carrier density is equal to the background doping concentration ( $\mathrm{N}_{\mathrm{d}}$ for $\mathrm{n}$-type, $\mathrm{N}_{\mathrm{a}}$ for $\mathrm{p}$-type) of the semiconductor. Next the conditions for the occurrence of strong inversion are derived.

For an n-type semiconductor, as shown in Fig. 2.1(b), the condition of strong inversion at the interface would result in $E_{F}$ to lie below the $E_{F I}$ with an energy difference $q \psi_{\mathrm{BI}} \geqslant q \psi_{\mathrm{B}}$, where $q \psi_{\mathrm{B}}=\mathrm{kT} \cdot \ln \left(\mathrm{N}_{\mathrm{d}} / \mathrm{n}_{\mathrm{i}}\right)$. The latter represents the relative position of $E_{F}$ from $E_{F I}$ in the bulk region as defined by the background doping. This leads to the condition that at the interface $\phi_{\mathrm{b}} \geqslant \mathrm{u}_{\mathrm{T}} \cdot \ln \left(\mathrm{N}_{\mathrm{c}} / \mathrm{n}_{\mathrm{i}}\right)+\psi_{\mathrm{B}}$. Therefore for strong inversion and thus bipolar pn junction like behavior, the following conditions hold.

For an n-type semiconductor:

$$
\phi_{\mathrm{b}}=\left(\phi_{\mathrm{m}}-\chi_{\mathrm{s}}\right) / \mathrm{q} \geqslant \mathrm{u}_{\mathrm{T}} \cdot \ln \left(\frac{\mathrm{N}_{\mathrm{d}}}{\mathrm{n}_{\mathrm{i}}}\right)+\mathrm{u}_{\mathrm{T}} \cdot \ln \left(\frac{\mathrm{N}_{\mathrm{c}}}{\mathrm{n}_{\mathrm{i}}}\right),
$$

and for a p-type semiconductor:

$$
\phi_{\mathrm{b}}=\left(\mathrm{E}_{\mathrm{g}}-\phi_{\mathrm{m}}+\chi_{\mathrm{s}}\right) / \mathrm{q} \geqslant \mathrm{u}_{\mathrm{T}} \cdot \ln \left(\frac{\mathrm{N}_{\mathrm{a}}}{\mathrm{n}_{\mathrm{i}}}\right)+\mathrm{u}_{\mathrm{T}} \cdot \ln \left(\frac{\mathrm{N}_{\mathrm{v}}}{\mathrm{n}_{\mathrm{i}}}\right) .
$$

Using Boltzmann's approximation ( $\mathrm{n} \ll \mathrm{N}_{\mathrm{c}}$ or $\mathrm{p} \ll \mathrm{N}_{\mathrm{v}}$ ), the hole (electron) concentration in n-type (p-type) semiconductors can be expressed as:

$$
p=n_{i} \cdot \exp \left(-\frac{\psi_{B I}}{u_{T}}\right)=n_{i} \cdot \exp \left(\frac{\phi_{b}-E_{g} / 2 q}{u_{T}}\right) \sqrt{\frac{N_{v}}{N_{c}}} .
$$




$$
\mathrm{n}=\mathrm{n}_{\mathrm{i}} \cdot \exp \left(\frac{\psi_{\mathrm{BI}}}{\mathrm{u}_{\mathrm{T}}}\right)=\mathrm{n}_{\mathrm{i}} \cdot \exp \left(\frac{\phi_{\mathrm{b}}-\mathrm{E}_{\mathrm{g}} / 2 \mathrm{q}}{\mathrm{u}_{\mathrm{T}}}\right) \sqrt{\frac{\mathrm{N}_{\mathrm{c}}}{\mathrm{N}_{\mathrm{v}}}} .
$$

The above equations indicate that for a suitable $\phi_{\mathrm{m}}$, that governs $\phi_{\mathrm{b}}$, charge carriers of opposite polarity type from the background doping can be induced near the interface of a 1-D Schottky contact. This creates a very shallow p-n junction near the MS interface where drift-diffusion or possibly even BTBT governs the current, which is different from conventional SB based devices where $\phi_{\mathrm{b}}$ at the interface solely controls the emission of charge carriers.

In case of an intrinsic semiconductor where $\psi_{\mathrm{B}}=0$, Eqs. (2.3)-(2.4) can be transformed into the following equations for $\mathrm{p}$-type doping:

$$
\phi_{\mathrm{m}} \geqslant x_{\mathrm{s}}+\frac{\mathrm{E}_{\mathrm{g}}}{2}
$$

and n-type doping:

$$
\phi_{\mathrm{m}} \leqslant \chi_{\mathrm{s}}+\frac{E_{\mathrm{g}}}{2} .
$$

By using metals with different work functions, it is possible to make an intrinsic semiconductor p-type or n-type locally via the ED approach. The term $\chi_{s}+\left(E_{g} / 2\right)$ is essentially the position of Fermi level in the intrinsic semiconductor, i.e. its work function $\phi_{\mathrm{s}}$.

Experimentally, inversion layer effects in high-barrier Schottky diodes have been reported before in $[55,94]$. Recently, such 1-D Schottky based p$\mathrm{n}$ junctions have also been reported using TCAD simulations for a vertical bipolar junction transistor (BJT) structure [95] and for a GaAs tunnel diode [96]. For the tunnel diode however Fermi-Dirac statistics should be applied and consequently Eqs. (2.5)-(2.6) do not hold. Nonetheless, a suitable $\phi_{\mathrm{m}}$ is still required for a $\mathrm{p}-\mathrm{n}$ junction formation. The physics of such high-barrier diodes will be discussed in more detail in chapter 3-4 of this thesis.

\section{Gated Schottky based UTB devices}

In this section, the ED concept is discussed in devices in which a dielectric layer is placed between the metal and semiconductor body. In addition, the semiconductor device makes use of a fully depleted (FD) UTB devoid of any depletion charge [97]. Fig. 2.5 shows a schematic cross-section of the system under discussion which is similar to an FD 2-D MOS system.

The potential drop across the device perpendicular to the gate electrode is given by the following equation:

$$
V_{\mathrm{GB}}=\mathrm{V}_{\mathrm{ox}}+\psi_{\mathrm{s}}+\phi_{\mathrm{m}} / \mathrm{q}-\chi_{\mathrm{s}} / \mathrm{q}-\left(\mathrm{E}_{\mathrm{c}}-\mathrm{E}_{\mathrm{FI}}\right) / \mathrm{q}
$$

where $V_{\mathrm{GB}}$ is the applied gate potential difference between gate and semiconductor body, $V_{o x}$ is the potential drop across the oxide and $\psi_{\mathrm{s}}$ is the surface potential i.e. shift in the Fermi-level from its intrinsic position. For 

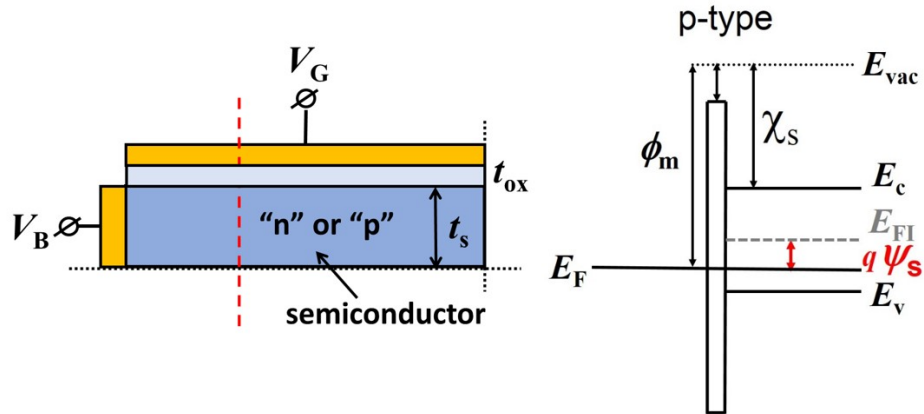

metal insulator semiconductor

Figure 2.5: Schematic cross-section of a UTB device with 2-D gated Schottky contact for work function or bias-induced ED (left). Schematic energy band diagram for a p-type formed region perpendicular to the gate along the red dashed line (right).

not too high carrier concentrations $\left(n, p \ll N_{c}, N_{v}\right), V_{\text {ox }} \approx 0$ for an FD UTB with ideal interfaces, and $\mathrm{V}_{\mathrm{GB}}=0$ for a purely work function induced $\mathrm{ED}$ case.

Then it can be obtained that

$$
\psi_{\mathrm{s}}=\left(E_{\mathrm{FI}}-E_{\mathrm{F}}\right) / \mathrm{q}=\chi_{\mathrm{s}} / \mathrm{q}-\phi_{\mathrm{m}} / \mathrm{q}-\mathrm{u}_{\mathrm{T}} \cdot \ln \left(\frac{\mathrm{n}_{\mathrm{i}}}{\mathrm{N}_{\mathrm{c}}}\right) .
$$

Eq. (2.10) is the condition for ED in a UTB gated Schottky system which is equivalent to Eq. (2.7) for p-type doping $\left(\psi_{\mathrm{s}}<0\right)$ and to Eq. (2.8) for n-type doping $\left(\psi_{\mathrm{s}}>0\right)$. Analogous to the $\mathrm{p}-\mathrm{n}$ junction formation in a 1-D Schottky contact, the concentration of the induced charge can be expressed as:

$$
\begin{aligned}
& p=n_{i} \exp \left(-\frac{\psi_{s}}{u_{T}}\right), \\
& n=n_{i} \exp \left(\frac{\psi_{s}}{u_{T}}\right) .
\end{aligned}
$$

Fig. 2.6(a) shows the induced hole density against the oxide thickness for an $\mathrm{Si} / \mathrm{SiO}_{2}$ gated Schottky structure (Fig. 2.5) with $\phi_{\mathrm{m}}=5.1 \mathrm{eV}$. Eq. (2.10) indicates that the induced charge carrier density is independent of insulator and semiconductor thicknesses for UTB devices as $V_{\mathrm{ox}} \approx 0$ in our simplified model. However TCAD [98 $]^{2}$ produces somewhat different results. This is attributed to the presence of a high amount of mobile charge resulting in a finite $V_{o x}\left(\sim 30 \mathrm{mV}\right.$ at $t_{o x}=6 \mathrm{~nm}$ and $\left.t_{\mathrm{s}}=10 \mathrm{~nm}\right)$. Still, the model is useful for studying trends. For more accurate results, a numerical model can be derived [99], which is beyond the scope of this chapter.

\footnotetext{
${ }^{2}$ TCAD simulations wherever referred throughout this thesis are performed with Sentaurus device simulator software [98] unless stated otherwise.
} 

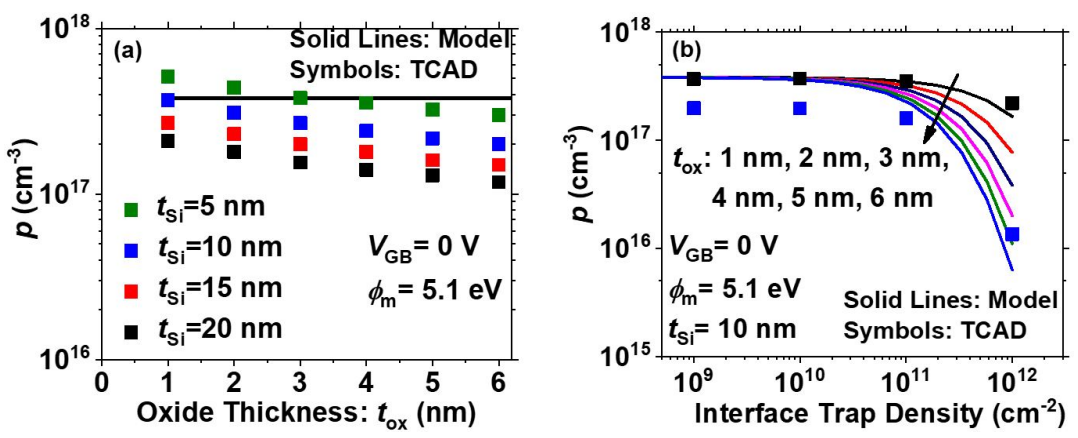

Figure 2.6: Induced hole concentration for an $\mathrm{Si} / \mathrm{SiO}_{2}$ gated Schottky structure (see Fig. 2.5) with $\phi_{\mathrm{m}}=5.1 \mathrm{eV}$ for (a) varying oxide thicknesses with ideal interfaces and (b) varying interface trap densities. In the TCAD simulations, a uniform distribution of both donor and acceptor traps across the bandgap [1] has been assumed. The carrier density was extracted at a lateral distance of $0.5 \mu \mathrm{m}$ from the Schottky side contact.

Now taking into account the effect of the interface trap charge $\left(Q_{i t}\right)$, Eq. (2.10) can be modified as:

$$
\psi_{\mathrm{s}}=\chi_{\mathrm{s}} / \mathrm{q}-\phi_{\mathrm{m}} / \mathrm{q}-\mathrm{u}_{\mathrm{T}} \cdot \ln \left(\frac{\mathrm{n}_{\mathrm{i}}}{\mathrm{N}_{\mathrm{c}}}\right)+\left(\frac{\mathrm{Q}_{\mathrm{it}}}{\mathrm{C}_{\mathrm{ox}}}\right),
$$

with $\mathrm{Q}_{\mathrm{it}}=-\mathrm{C}_{\mathrm{it}} \psi_{\mathrm{s}}$ (assuming a uniform interface trap density) where $\mathrm{C}_{\mathrm{ox}}=\epsilon_{\mathrm{ox}} / \mathrm{t}_{\mathrm{ox}}$ and $\mathrm{C}_{\mathrm{it}}=\mathrm{q}^{2} \mathrm{D}_{\mathrm{it}}$ are the oxide and interface trap capacitance per unit area respectively, and $\mathrm{D}_{\mathrm{it}}$ is the interface trap density [1]. Rearranging the terms in Eq. (2.13) gives the following condition for the work function induced ED including the effect of interface states:

$$
\psi_{\mathrm{s}}=\frac{\left(\chi_{\mathrm{s}} / \mathrm{q}-\phi_{\mathrm{m}} / \mathrm{q}-\mathrm{u}_{\mathrm{T}} \cdot \ln \left(\frac{\mathrm{n}_{\mathrm{i}}}{\mathrm{N}_{\mathrm{c}}}\right)\right) \cdot \mathrm{C}_{\mathrm{ox}}}{\left(\mathrm{C}_{\mathrm{it}}+\mathrm{C}_{\mathrm{ox}}\right)} .
$$

Eq. (2.14) along with Eqs. (2.11)-(2.12) expresses the essential condition for induced ED in a gated Schottky device with interface trap charge. It shows that the ED reduces with an increase in interface trap density. From Fig 2.6(b), where the induced charge concentration is plotted against the interface trap density using Eq. (2.14), it can be seen that the effect of interface traps is more pronounced for a larger oxide thickness. The charge concentration is reduced by a maximum of two orders in magnitude when the trap density is increased to $10^{12} / \mathrm{cm}^{2}$ for an oxide thickness of $6 \mathrm{~nm}$.

The physics of gated Schottky contacts discussed above lays the foundation for the charge plasma concept as applied to FD UTB devices such as the TFET. This is discussed in the next paragraph.

\section{Charge Plasma Devices}

Hueting et al. [60,61] proposed to adopt two different work function metals to induce different polarity of charge carriers in the semiconductor. The 

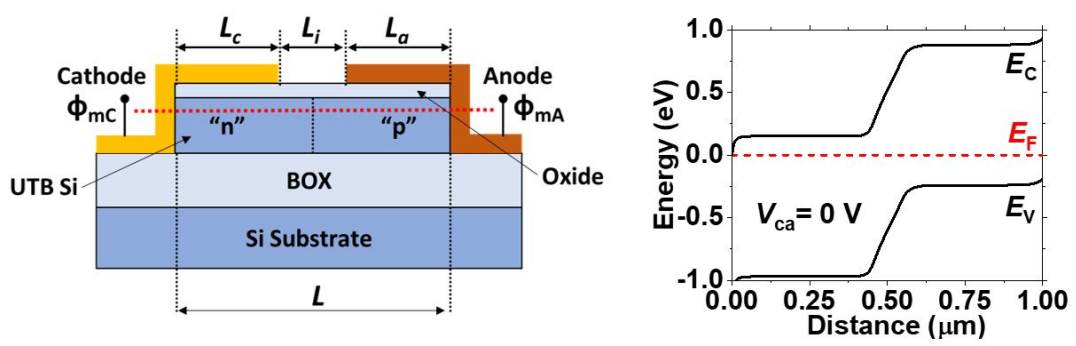

Figure 2.7: Schematic cross section of the CP p-n diode (left) and its simulated energy band diagram along the dotted red line at equilibrium (right). $\phi_{\mathrm{mC}}=4.17 \mathrm{eV}$ and $\phi_{\mathrm{mA}}=5.1 \mathrm{eV}$ are cathode and anode metal work functions respectively. $\mathrm{L}_{\mathrm{c}}$ and $\mathrm{L}_{\mathrm{a}}$ denote cathode and anode gate lengths respectively. $\mathrm{L}_{\mathrm{i}}$ denotes the length of the intrinsic region i.e. the gap between the two electrodes. The thickness of UTB Si $\left(t_{S i}\right)$ is $20 \mathrm{~nm}$ and gate oxide thickness $\left(t_{\mathrm{ox}}\right)$ is $5 \mathrm{~nm}$. [60].

schematic cross section of the so-called charge plasma (CP) p-n junction diode is shown in Fig. 2.7. The CP diode comprises a gated anode and a gated cathode with metals of different $\phi_{\mathrm{m}}$. From Eqs. (2.10)-(2.12) it can be concluded that for the anode region a high $\phi_{\mathrm{m}}$ is required, while the opposite holds for the cathode region.

Fig. 2.7 also shows the energy band diagram of a CP diode along the lateral direction as obtained from 2-D TCAD simulations indicating that a $p-n$ junction has formed. The band diagram is similar to a typical chemically doped p-n junction with some key differences which were also highlighted in [74]. The quasi-neutral regions of this diode are formed by the ED gates. A typical feature of the lateral ED configuration is that the potential distribution is linear in the intrinsic gap region because of the absence of impurities, unlike a conventional p-n junction which has a parabolic potential profile in the depletion region. The built-in electric field in an impurity doped p-n junction is formed by fixed donor or acceptor ions near the junction to balance drift and diffusion components at equilibrium. This built-in field also exists in the $\mathrm{CP}$ p-n diode but is primarily formed by the work function difference at the edges of the gated regions. Further, the band bending near the electrodes is attributed to the 2-D fringe field effect. Analytical drift-diffusion models for the I - V curves in the CP p-n diode [100] also showed good agreement with TCAD simulations.

In the first experimental realization [61], Pd was employed for the anode contact and Er for the cathode. The fabricated device showed good rectifying behavior with a low constant leakage current of $1 \mathrm{fA} / \mu \mathrm{m}$ and an on/off-current ratio of around $10^{7}$ at $V_{D}=1 \mathrm{~V}$ forward bias and room temperature. Fig. 2.8(a) shows measured ${ }^{3} \mathrm{I}-\mathrm{V}$ characteristics of various fabricated $\mathrm{CP}$ diodes with different combinations of anode and cathode metals. The experimental results indicate that both the hole and electron current are important for the $\mathrm{CP}$ diode. The current increases as the anode

${ }^{3}$ The CP diode fabrication and measurements were performed by B. Rajasekharan [61]. 


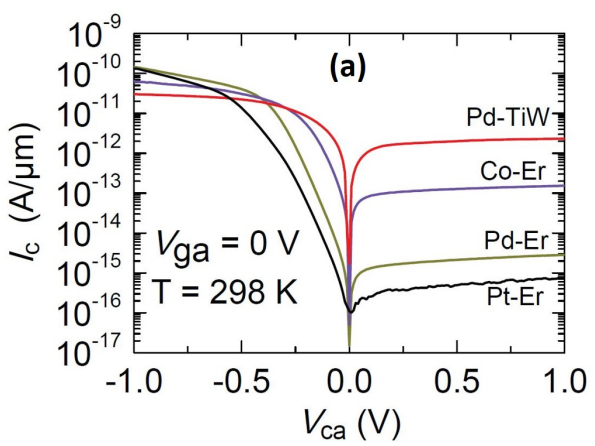

(b)

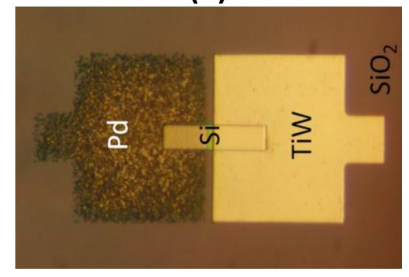

Figure 2.8: (a) $\mathrm{I}_{\mathrm{c}}-\mathrm{V}_{\mathrm{ca}}$ characteristics for various valence and conduction level metal combinations $\mathrm{Pt}-\mathrm{Er}, \mathrm{Pd}-\mathrm{Er}, \mathrm{Co}-\mathrm{Er}$ and $\mathrm{Pd}-\mathrm{TiW}$ on either side of silicon. $\mathrm{L}_{\mathrm{a}}=\mathrm{L}_{\mathrm{c}}=0.9 \mu \mathrm{m}, \mathrm{L}_{\mathrm{i}}=3 \mu \mathrm{m}$ (see Fig.2.7). The substrate potential $\left(\mathrm{V}_{\mathrm{ga}}\right)$ was kept at $0 \mathrm{~V}$. (b) Top view of a realized CP diode showing the effect of placing a wafer with high work function (Pd) and low work function (TiW) metal islands in a chemical etching solution. The combination of the metals with the solution formed an electrolyte cell which resulted in the corrosion of highest work function (Pd) metal [61]. Image courtesy: B. Rajasekharan.

work function is reduced by replacing Pt with Pd or Co, or conversely, by increasing the cathode work function by replacing Er with TiW. By using the same metal for anode and cathode contact and the substrate as back gate, MOSFET characteristics were obtained [61].

The CP based ED concept has been extended to various other devices. Kumar and Nadda [62] proposed and investigated the CP based lateral BJT by replacing the doped emitter, base and collector regions of a conventional bipolar device with ED regions using metals with different work functions as shown in Fig. 2.9(a). The authors proposed $\mathrm{Hf}\left(\phi_{\mathrm{mE}}=3.9 \mathrm{eV}\right)$ for the emitter, $\mathrm{Pt}\left(\phi_{\mathrm{mB}}=5.65 \mathrm{eV}\right)$ for the base, and $\mathrm{Al}\left(\phi_{\mathrm{mC}}=4.28 \mathrm{eV}\right)$ for the collector electrode, thereby achieving an $\mathrm{n}^{+}-\mathrm{p}-\mathrm{n}$ configuration. The results obtained from 2-D TCAD simulations show similar device characteristics but a higher current gain compared to the conventional impurity doped counterpart with the same dimensions.

Another device based on the CP concept is the doping-less TFET, proposed by Kumar and Janardhanan [63], shown in Fig. 2.9(b). Similar to the CP-BJT, again the impurity doped regions of the conventional TFET were replaced with ED regions by employing metals with a different work function: $\mathrm{Hf}$ for the n-type drain and $\mathrm{Pt}$ for the p-type source. The performance of the $\mathrm{CP}$ based doping-less TFET was found to be similar to the conventionally doped TFET with the same device geometry.

Other proposed CP-devices are summarized in Table 2.1. So far most of these architectures have not been experimentally realized. Similar ideas for graphene doping via metal contacts have also been reported [70-73, 101103]. 


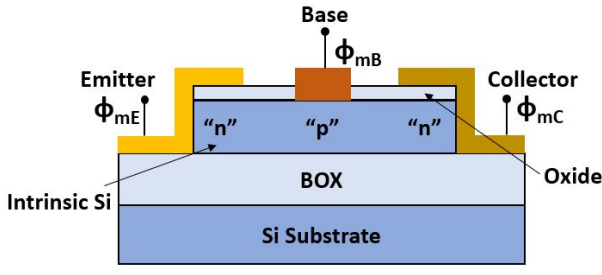

(a)

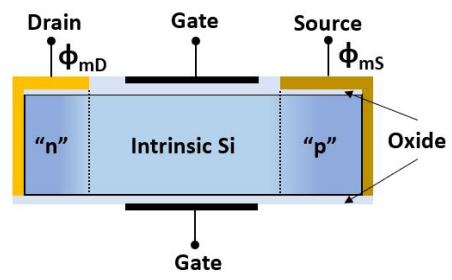

(b)

Figure 2.9: Schematic cross section of the CP based (a) Lateral BJT [62], (b) TFET [63]. The " $n$ " and "p" indicate electrostatically doped regions.

\subsubsection{Bias-induced ED}

The applied electric field at the MS interface also plays a decisive role in governing the electrostatics and thereby charge induction in the UTB semiconductor. It can be argued whether the principle of bias-induced ED is not simply based on the conventional field-effect. However, in conventional FETs, the charge carriers originate from doped semiconductor regions in close vicinity (like doped S/D regions), whereas in the ED concept the charge carriers originate from a metal electrode which is in direct contact with the semiconductor body ${ }^{4}$. Hereby, Fig. 2.5 is again referred. A similar expression as derived previously for the work function induced ED (Eq. (2.10)), however with an additional term to account for non-zero $\mathrm{V}_{\mathrm{GB}}$, is obtained for bias-induced $\mathrm{ED}$ case:

$$
\psi_{\mathrm{s}}=\mathrm{V}_{\mathrm{GB}}+\chi_{\mathrm{s}} / \mathrm{q}-\phi_{\mathrm{m}} / \mathrm{q}-\mathrm{u}_{\mathrm{T}} \cdot \ln \left(\frac{\mathrm{n}_{\mathrm{i}}}{\mathrm{N}_{\mathrm{c}}}\right) .
$$

For a bulk or partially depleted channel, similar relations can be obtained using a non-zero $V_{o x}$ term in Eq. (2.9). The term $\left(\chi_{s} / q-\phi_{m} / q-u_{T}\right.$. $\left.\ln \left(n_{i} / N_{c}\right)\right)$ is actually the work function difference $\left(\phi_{m}-\phi_{s}\right) / q$, which is zero in case of a purely bias-induced ED. In this case it is easy to understand that a positive $V_{G B}$ will induce $n$-doping while a negative $V_{G B}$ will induce p-doping. Further, the effect of interface traps can be accounted for as

$$
\psi_{\mathrm{s}}=\mathrm{V}_{\mathrm{GB}}+\chi_{\mathrm{s}} / \mathrm{q}-\phi_{\mathrm{m}} / \mathrm{q}-\mathrm{u}_{\mathrm{T}} \cdot \ln \left(\frac{\mathrm{n}_{\mathrm{i}}}{\mathrm{N}_{\mathrm{c}}}\right)+\left(\frac{\mathrm{Q}_{\mathrm{it}}+\mathrm{Q}_{\mathrm{inv}}}{\mathrm{C}_{\mathrm{ox}}}\right),
$$

with $\mathrm{Q}_{\text {inv }}$ is the mobile inversion charge. Note that earlier modeling work on I-V curves of asymmetric dual-gate (DG) devices [99] could be used to derive a model for bias-induced ED including traps.

The simultaneous induction of p-type and n-type charged regions in a semiconductor body via an applied field can be realized using a dual (or multiple) gate structure [48]. By biasing two gates with opposite polarities, electrons and holes can be simultaneously induced in a semiconductor

\footnotetext{
${ }^{4}$ In the absence of any direct metal contact with a suitable work function, the supply of charge carriers is limited by thermal generation rate (refer to chapter 5). Alternatively, doped regions can be employed for carrier supply [48].
} 


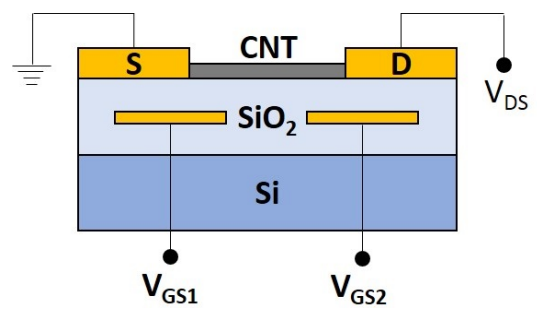

Figure 2.10: Schematic cross section of the CNT p-n diode [74]. The split gate configuration was used to electrostatically induce a lateral p-n junction. Biasing $\mathrm{V}_{\mathrm{GS} 1}<0$ would give a p-type region in the adjacent $\mathrm{CNT}$ channel and $\mathrm{V}_{\mathrm{GS} 2}>0$ would result in an n-type region.

body as analytically explained by Eqs. (2.15) and (2.16). Depending on the relative position of such gates, electrostatically induced local bipolar regions have been reported both in lateral [74] and in vertical structures [79] as discussed in the next paragraph.

\section{Lateral p-n Junction}

Bias-induced ED was first experimentally demonstrated in a CNT system [74] where a lateral p-n junction diode was formed adopting a split gate configuration as shown in Fig. 2.10. Biasing a first gate electrode with a positive voltage with respect to the CNT body resulted in an n-type doping of a CNT channel region adjacent to it. Similarly a negative voltage with respect to the CNT body at a second gate resulted in p-type doping. This led to the formation of a lateral p-n junction diode in the CNT channel. The experimentally fabricated device showed rectifying characteristics of a p-n junction diode with an ideality factor close to one. In addition, the same polarity biases at both gate electrodes resulted in an n-channel or p-channel FET. BTBT was also observed in CNTs [104] demonstrating an ED p-n junction which could be interesting for the TFET.

Recently, the concept of bias-induced ED was also experimentally applied in an advanced and mature FD-SOI process to demonstrate the reconfigurable device [68] which can be configured into nine types of devices with different functionalities such as virtual diodes, TFETs, p-i-n diodes and band-modulation FETs. For more detailed overview on this and related work, please refer to [48].

\section{Vertical p-n Junction: Electron-Hole Bilayer (EHB)}

An opposite polarity gate bias configuration in the vertical direction can be utilized to form a bias-induced electron-hole bilayer (EHB) as illustrated in Fig. 2.11. This was demonstrated in $[105,106]$ using a dual-gate $\mathrm{SiO}_{2} / \mathrm{Si} / \mathrm{SiO}_{2}$ system.

In case of a relatively thick semiconductor with a partially depleted channel, the two metal gates do not have any electrostatic coupling and 

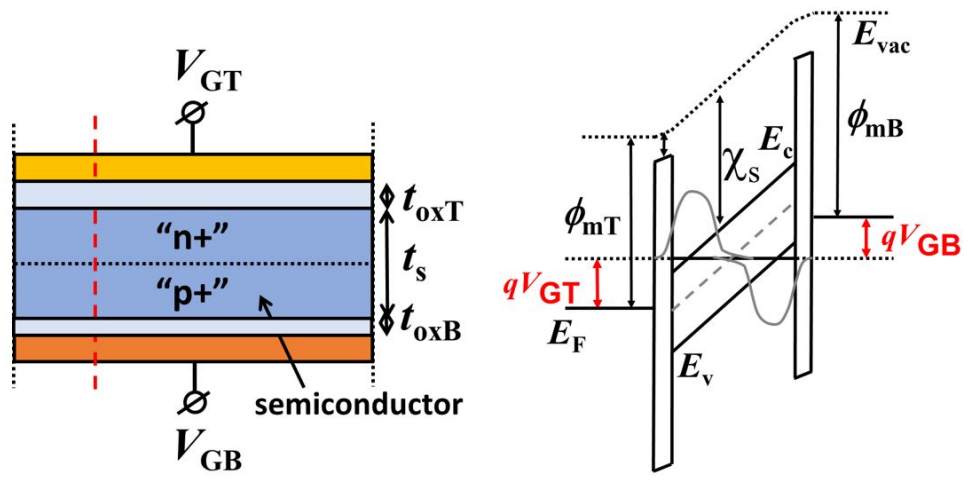

Figure 2.11: Schematic cross-section of the electron-hole bilayer concept (left). Schematic energy band diagram perpendicular to the gates along the red dashed line (right). By enforcing an opposite polarity bias between the top and bottom gate a tunnel junction can be formed. The wavefunction curves represent the electron (left) and hole (right) distribution in the EHB structure for illustration purposes.

therefore the bias-induced charge near each gate can be independently described using Eq. (2.15) (for $n, p \ll N_{c}, N_{v}$ ). In case of a dual asymmetric gate structure with an FD UTB channel however, the electrostatic interaction between the two gates needs to be taken into account. Analogous to the previous work of Lim and Fossum [107] and assuming a not too high charge carrier concentration, the following equation is derived for the vertical electric field in the semiconductor body for the EHB concept in the coupled dual gate system (see appendix A):

$$
E_{\mathrm{s}}=\frac{\left(\mathrm{V}_{\mathrm{GT}}-\mathrm{V}_{\mathrm{GB}}\right)-\left(\phi_{\mathrm{mT}}-\phi_{\mathrm{mB}}\right)}{\frac{\varepsilon_{\mathrm{s}}}{\varepsilon_{\mathrm{ox}}}\left(\mathrm{t}_{\mathrm{oxT}}+\mathrm{t}_{\mathrm{oxB}}\right)+\mathrm{t}_{\mathrm{s}}},
$$

where the subscripts $\mathrm{T}$ and $\mathrm{B}$ refer to the top and bottom of the structure, respectively. Fig. 2.12(a) plots $E_{s}$ against the semiconductor body thickness $\mathrm{t}_{\mathrm{s}}$ for an $\mathrm{HfO}_{2} / \mathrm{Si} / \mathrm{HfO}_{2} \mathrm{EHB}$ structure. The figure shows that TCAD simulations are in good agreement with Eq. (2.17). Semi-classically, there will be an induced electron density near the top gate electrode if $\psi_{\mathrm{ST}}>0$ (refer to Eq. (A.6)) with an electron density at the interface $n=n_{i} \exp \left(\psi_{\mathrm{sT}} / \mathrm{u}_{\mathrm{T}}\right)$. Similarly if $\psi_{\mathrm{sB}}<0$ (refer to Eq. (A.7)) there will be an induced hole density near the bottom gate electrode at the interface $p=n_{i} \exp \left(-\psi_{\mathrm{sB}} / \mathfrak{u}_{\mathrm{T}}\right)$. Obtaining closed form solutions for $\psi_{\mathrm{sT}}$ and $\psi_{\mathrm{sB}}$ is however rather difficult. Moreover, quantum effects can change this picture [108] as qualitatively shown in Fig. 2.12(b) using TCAD simulation. The effect of fixed or interface charge can also be accounted for as described by Eq. (A.9) (see appendix $\mathrm{A}$ ) by assuming non-zero $\mathrm{Q}_{\mathrm{fT}}$ and $\mathrm{Q}_{\mathrm{fB}}$.

Lattanzio et al. [79, 80] have applied the EHB concept to a DG-TFET geometry. This transistor was conventionally symmetrically biased for better electrostatic control over the channel. Under asymmetric bias, a biasinduced EHB forms in the channel. For a certain top-bottom bias difference 

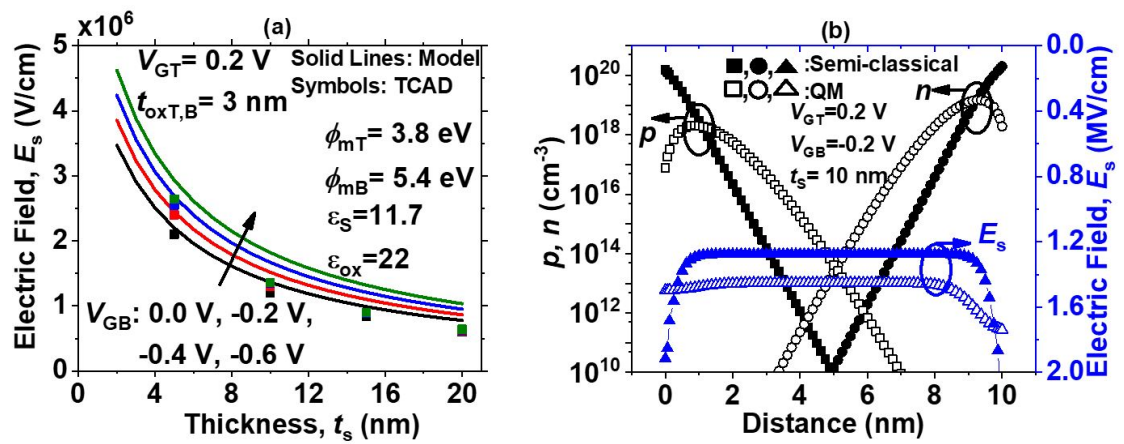

Figure 2.12: (a) The vertical electric field in the $\mathrm{HfO}_{2} / \mathrm{Si} / \mathrm{HfO}_{2} \mathrm{EHB}$ structure (refer to Fig. 2.11) for various semiconductor body thicknesses. Quantum Mechanical (QM) effects have not been taken into account both in the model (Eq. (2.17)) and TCAD simulation [98]. (b) TCAD simulation of charge carrier distribution and electric field profile in the vertical direction along the red dashed line of Fig. 2.11 with $t_{\mathrm{s}}=10 \mathrm{~nm}$ and $\mathrm{V}_{\mathrm{GT}}=0.2 \mathrm{~V}$ and $\mathrm{V}_{\mathrm{GB}}=-0.2 \mathrm{~V}$ shown using both semi-classical and QM (Density-gradient model [109]) approaches. The detailed quantitative calibration of the QM model in TCAD is not the focus of this work.

( 0.1-0.5 V), BTBT of charge carriers in the EHB p-n junction occurs which results in an increase in the drain current [108]. In this case the direction of tunneling is parallel to the gate field. This results in more on-state tunneling current compared to e.g. the doping-less TFET [63] because of the large tunneling surface area and a stronger electrostatic control over both n-type and p-type regions in the subthreshold region. However, there has been some debate on the effectiveness of the EHB formation [81, 110]. In [81] experimentally realized EHB-TFET structures were reported and it was argued that conditions to meet efficient BTBT and the formation of the EHB cannot simultaneously be satisfied: there is a trade-off between the induced field and quantum-confinement.

The use of EHB concept for undoped light-emitting device application $[57,82]$ will be discussed in chapter 5 .

\subsection{Electrostatic Doping Approaches: Discussion}

Table 2.1 provides a summary and classification of the various ED device concepts. From the literature, it can be deduced that a high carrier density of both $\mathrm{p}$ and n-type $\left(10^{18}-10^{20} \mathrm{~cm}^{-3}\right)$ can be electrostatically induced in UTB devices, particularly for narrow bandgap semiconductors. Fig 2.13 shows the calculated induced charge carrier concentration using Eq. (2.10)(2.12) for gated Schottky regions of the UTB CP device shown in Fig. 2.7.

For electrons, ED is mainly governed by the electron affinity of the semiconductor and the metal work function, $\phi_{\mathrm{m}}$. Therefore, obtaining a high electron density is relatively easy by adopting a suitable elemental metal with low $\phi_{\mathrm{m}}$ such that Eq. (2.8) is satisfied. The $\phi_{\mathrm{m}}$ of available 
Table 2.1: Summary of various reported electrostatically doped materials and device concepts till date. The notation (exp.) denotes experimentally demonstrated concept while others have been investigated via modeling and TCAD simulation only.

\begin{tabular}{|c|c|c|c|}
\hline Material & SB based devices & $\begin{array}{l}\text { Work function in- } \\
\text { duced ED }\end{array}$ & $\begin{array}{l}\text { Bias- } \\
\text { induced } \\
\text { ED }\end{array}$ \\
\hline 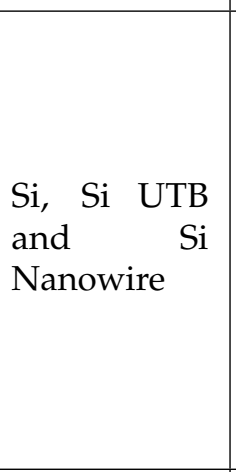 & 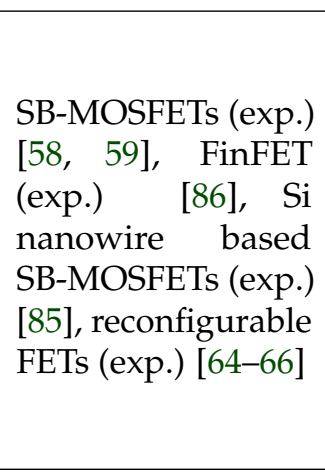 & $\begin{array}{l}\text { CP based p-n diode } \\
\text { (exp.) [60, 61], BJTs } \\
\text { [62, 95, 111, 112], } \\
\text { TFET [63], IMOS } \\
\text { [113], Junctionless } \\
\text { transistor [114], } \\
\text { Biristor [115], } \\
\text { SiGe-on-insulator } \\
\text { MOSFET [116], } \\
\text { graded channel } \\
\text { MOSFET [117] }\end{array}$ & $\begin{array}{l}\text { EH bi- } \\
\text { layer TFET } \\
\text { [79, 80], EH } \\
\text { bilayer TFET } \\
\text { (exp.) [81], } \\
\text { virtual diode } \\
\text { (exp.) [67], } \\
\text { Esaki diode } \\
\text { (exp.) [69] }\end{array}$ \\
\hline CNT & $\begin{array}{l}\text { SB-MOSFETs } \\
{[75,76,87], \text { Light }} \\
\text { emitting SB-FET } \\
\text { (exp.) [89] }\end{array}$ & & $\begin{array}{lr}\begin{array}{l}\text { p-n } \\
\text { (exp.) }\end{array} & \text { diode } \\
{[17,} & 74], \\
\text { FET } & \text { (exp.) } \\
{[104]} & \\
\end{array}$ \\
\hline Graphene & & Graphene ED [72] & $\begin{array}{l}\text { Graphene } \\
\text { ED [101], } \\
\text { Graphene } \\
\text { FET (exp.) } \\
{[70,71,73,} \\
102,103]\end{array}$ \\
\hline $\begin{array}{l}\text { III-V materi- } \\
\text { als }\end{array}$ & $\begin{array}{l}\text { GaN n-type SB- } \\
\text { MOSFET (exp.) } \\
{[51]}\end{array}$ & $\begin{array}{lr}\text { GaAs } & \text { Tunnel } \\
\text { diode [96], GaN } \\
\text { n-MOSFET } \\
\text { InAs TFET [118] }\end{array}$ & $\begin{array}{l}\text { GaAs EH } \\
\text { Bilayer (exp.) } \\
{[119] \text { InAs }} \\
\text { EH bilayer } \\
\text { LED }[57,82]\end{array}$ \\
\hline $\begin{array}{l}\text { Polycrystalline } \\
\text { materials }\end{array}$ & & $\begin{array}{l}\text { CP based poly-Si } \\
\text { TFT [120], IGZO } \\
\text { TFT (exp.) [121] }\end{array}$ & \\
\hline $\begin{array}{l}\text { 2-D materi- } \\
\text { als }\end{array}$ & $\begin{array}{l}\text { n-type, p-type } \\
\text { SB-MOSFET (exp.) } \\
{[122,123]}\end{array}$ & & $\begin{array}{l}2-\mathrm{D} \text { transis- } \\
\text { tors }[77,78]\end{array}$ \\
\hline
\end{tabular}

elemental metals varies roughly between $2.14 \mathrm{eV}$ for Cesium (Cs) and 5.65 $\mathrm{eV}$ for Platinum $(\mathrm{Pt})[1,125]$.

On the other hand, obtaining a high hole density is rather difficult, particularly for wide bandgap semiconductors, as large bandgap raises the demand for high $\phi_{\mathrm{m}}$ metal (see Eq. (2.7)). For example, in case of $\mathrm{GaN}$ with $\mathrm{E}_{\mathrm{g}}=3.4 \mathrm{eV}$ and electron affinity $\chi_{\mathrm{s}}=4.1 \mathrm{eV}$, the condition for the work function induced n-type ED i.e. $\phi_{m}<\left(\chi_{s}+E_{g} / 2\right)$ can be satisfied 


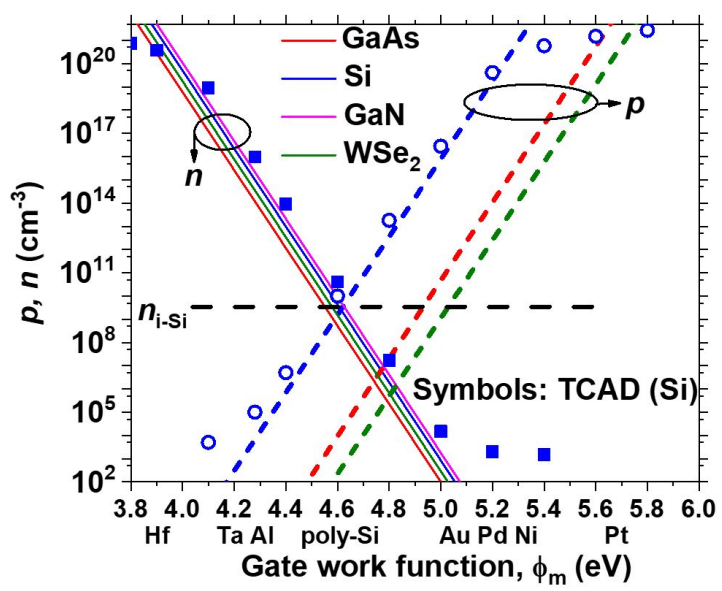

Figure 2.13: Calculated induced carrier concentration using Eq. (2.10)-(2.12) in "ntype" and "p-type" gated Schottky regions of the CP diode structure (refer to Fig. 2.7) with a varying $\phi_{\mathrm{m}}$ of the gate electrode. The calculation is performed for different semiconductor channel materials $\left(\mathrm{Si}\left(\mathrm{E}_{\mathrm{g}}=1.12 \mathrm{eV}, \chi_{s}=4.07 \mathrm{eV}\right), \mathrm{GaAs}\left(\mathrm{E}_{\mathrm{g}}=1.42 \mathrm{eV}\right.\right.$, $\left.\left.\chi_{s}=4.07 \mathrm{eV}\right), \mathrm{GaN}\left(E_{g}=3.39 \mathrm{eV}, \chi_{s}=4.1 \mathrm{eV}\right), W_{S e}\left(E_{g}=1.56 \mathrm{eV}, \chi_{s}=4.03 \mathrm{eV}\right)[124]\right)$ in the UTB device. TCAD simulation (symbols) for Si shows good agreement with the model.

with a lower $\phi_{\mathrm{m}}$ metal like Al. However similar p-type ED in GaN would require a $\phi_{\mathrm{m}}$ higher than $5.8 \mathrm{eV}$ (refer to Eq. (2.7)), which is not available from any elemental metal. A possible solution to this problem in wide bandgap semiconductors could be the use of extreme $\phi_{\mathrm{m}}$ materials other than traditional metal such as $\mathrm{MoO}_{x}[123,126]$ with reported $\phi_{m}$ as high as $6.6 \mathrm{eV} . \mathrm{MoO}_{\mathrm{x}}$ based contacts on $\mathrm{Si}$ will the subject of our investigation in chapter 4.

Among various reported ED devices discussed so far, the SB-MOSFET, the reconfigurable FET and the TFET based on FD semiconductors and 2-D materials appear to be the most promising for future CMOS ${ }^{5}$. The performance of ED devices with well-optimized interface electrostatics could be at par with impurity doped devices. However more extensive experimental investigation is required to confirm whether ED could replace conventional doping in mainstream Si-CMOS technology. Nevertheless, ED could be the potential solution for alternative materials systems like Graphene, CNTs, TMDs, nanowires where conventional impurity doping is a big challenge. The ED is also interesting for innovative device concepts such as EHB-TFETs and RFETs which could not be realized using conventional doping.

Although ED based approaches look very promising, there are still some limitations and drawbacks which need to be addressed from a technology perspective.

\footnotetext{
${ }^{5}$ For detail discussion on performance, technological aspects and merits of various potential ED-based devices for future CMOS, please refer to [46-48].
} 
First, the ED concept is usually designed assuming ideal MS contacts. However, there has been much experimental evidence that the observed SBHs $\left(\phi_{b}\right)$ and thus "effective" work functions on various MS systems deviate from their bulk values and the linear relationship between $\phi_{\mathrm{b}}$ and $\phi_{\mathrm{m}}$ may not hold in certain cases [83]. The assumption of an ideal, abrupt and non-reactive MS interface does not hold in practice. The chemical reactivity between a metal and a semiconductor, interdiffusion and the presence of interface dipoles make the observed $\phi_{\mathrm{b}}$ to deviate from the ideal Schottky-Mott relation [127] where $\phi_{\mathrm{b}}$ has a linear relationship with $\phi_{\mathrm{m}}$.

Recently, Tung [128] has reported a detailed review on physics and chemistry of Schottky barriers at MS interfaces. The author reviewed various strategies of modifying $\phi_{\mathrm{b}}$ at the MS interface by an insertion of a thin $(\approx 2 \mathrm{~nm}$ ) interfacial layer. This avoids chemical interdiffusion and also decouples the electron states in the semiconductor from the influence of the metal [83]. $\phi_{b}$ obtained from such an metal-insulator-semiconductor (MIS) system with a thin interfacial layer shows a much stronger dependence on the metal work function than an MS interface without any intentionally grown interfacial layer [128, 129]. Such a thin interfacial layer could be a solution for the realization of work function induced ED devices with a better control over $\phi_{\mathrm{b}}$ [130]. Moreover, the presence of additional dipoles from the interfacial layer opens up the possibility to further tune $\phi_{\mathrm{b}}$. Since, most ED devices can be realized via low temperature processing, the metastability of the interfacial layer should also not be a big concern.

More recently van der Waals MS junctions [131] have been demonstrated where atomically flat metal thin film were laminated onto 2-D semiconductors without any direct chemical bonding thereby resulting in the MS interface essentially free from defects and Fermi-level pinning. Such contacts could be a way forward to realize MS junction where the Schottky-Mott limit can be closely followed.

Second, metal work function variation could also be a concern [132, 133], particularly for metal nitride gates like TiN [134] due to their polycrystalline nature. This could have serious implications on the effectiveness and uniformity of work function induced ED and device variability.

Third, implementing several metals in galvanic contact may lead to corrosion during wet processing (see Fig. 2.8(b)). This effect was observed for several combinations of electrode metals [61]. Rather than wet etching, other metal patterning approaches are recommended, such as dry etching, damascene, or lift-off.

Fourth, the scaling approach for ED devices may differs from chemically doped ones as reported in the recent study by Ilatikhameneh et al. [77]. The atomistic simulations of such ED 2-D material transistor showed a different behavior from devices with same EOT (equivalent oxide thickness) but with varied physical oxide thicknesses. The authors therefore proposed a new scaling theory and design guidelines for such ED devices 
where the actual physical oxide/dielectric thickness is more critical unlike conventional transistors where scaling is regulated by the EOT. The authors proposed to employ ultra-thin wide bandgap oxides as gate dielectric for both optimized ED and suppressed gate leakage.

Fifth, ED in general is more effective, for inducing both electrons and holes, in narrow bandgap semiconductors as discussed before.

Further, in general for any material, the smallest lateral spacing between the gates is desired for optimized performance of ED devices [77] which is largely limited by the lithographic resolution. This becomes more critical in case of wide bandgap materials as the highly resistive intrinsic gap between the two ED regions may lead to a reduced on-current and very high turn-on voltages. Recently, Pan and Chui [118] proposed to overcome this limitation in their new ED-TFET design. Such ideas can be explored for other ED devices as well.

Finally, the presence of interface traps could adversely affect the effectiveness of ED as discussed before. The use of a thin interface layer is a potential solution to minimize the effect of interface traps on induced charge $[128,130]$.

\subsection{Conclusion}

In this chapter, various reported ED approaches were reviewed. Using basic 1-D electrostatic relations, the conditions for ED were established and simple expressions for induced charge carrier densities as applied to different ED approaches were derived. The derived expressions highlight the role of metal and semiconductor work functions, energy bandgap, electron affinity and applied electric field, and the interplay between them for the induced ED. From various reported results and the developed analytical understanding, it can be concluded that ED techniques can be utilized to induce high charge concentrations in UTB active devices. In general, ED is more effective for narrow bandgap semiconductors.

ED can be obtained by low temperature processing with a fewer lithography steps. The performance of ED devices is also found competitive with that of conventional counterparts especially for SB-MOSFETs, RFETs and TFETs. However, many of these device architectures, in particular TFETs, have only been studied via TCAD simulations. Experimental realization of such structures will assist in evaluating the true merit of these device ideas for alternative material systems and innovative device concepts for future CMOS.

The effective work function variation, presence of fixed charge, interface traps, processing with multiple metals and applicability to wide bandgap materials are identified as the current major limitations to ED approaches. These need to be extensively addressed for developing future CMOS technology. 


\title{
HIGH-BARRIER SI-SCHOTTKY \\ DIODES
}

\begin{abstract}
In this chapter, the presence of minority carriers and their role in charge carrier transport in silicon (Si) Schottky diodes with a high potential barrier at a metal-semiconductor (MS) interface is investigated. Using TCAD simulations along with an analytical model, it is shown that in a high-barrier diode with an induced inversion charge, the minority diffusion current becomes comparable (or even higher) than the majority carrier thermionic emission current which imparts bipolar characteristics to otherwise conventionally known unipolar Schottky diode. It is also shown, via TCAD simulations, that the diffusion component and therefore minority carrier injection efficiency becomes more pronounced in case of a reduced substrate Gummel number, downsized Schottky contact area and at elevated temperatures. Further, the role of minority carriers at low injection in a moderately high barrier $\mathrm{Al} / \mathrm{p}$-Si Schottky diode has been experimentally verified via a prior-reported 2-diode electrical test method and by measuring infrared electroluminescence. The improved minority carrier injection efficiency in high-barrier Schottky contacts could be utilized for bipolar device application as an alternative to conventional chemically doped counterparts.
\end{abstract}

This chapter is an extension of the publication in IEEE Transactions on Electron Devices [56]. 


\subsection{Introduction}

Conventional chemical doping of semiconductor devices via impurities has become more challenging for nanometer scale devices with an extremely high doping gradient $[2,3]$. Moreover, chemical doping is also not yet technologically matured for emerging material systems other than silicon (Si) such as wide bandgap semiconductors (e.g. GaN, $\mathrm{SiC}$ and $\mathrm{ZnO}$ ), carbon nanotubes (CNTs) [9-12] and emerging two-dimensional (2-D) materials (graphene, phosphorene, silicene and transition metal dichalcogenides (TMDs)) $[13,14]$ which are increasingly relevant for future electronic as well as optoelectronic devices. From this viewpoint, electrostatic doping (ED) as discussed in chapter-2 ([46], and references therein) is being extensively investigated to overcome the challenges of conventional doping approaches in dimensionally-scaled devices.

In this direction, the case of a shallow p-n like junction induced by a large metal-semiconductor (MS) work function difference in a one dimensional (1-D) Schottky diode was previously discussed [46, 95, 96]. In conventional Schottky barrier (SB) based devices, the current is governed by one or more Schottky contacts. The physics of an SB formed at the MS interface has been well described earlier [83]. There is a prevalent perception that at low injection the SB diode is a majority carrier unipolar device in which the current is solely governed by thermionic emission (TE) (e.g., [135]) which can be expressed as:

$$
\mathrm{J}_{\mathrm{TE}}=A^{*} \mathrm{~T}^{2} \exp \left(-\frac{\mathrm{q} \phi_{\mathrm{b}}}{\mathrm{kT}}\right)\left[\exp \left(\frac{\mathrm{q} \mathrm{V}}{\mathrm{kT}}\right)-1\right],
$$

where $\mathrm{J}_{\mathrm{TE}}$ is TE current density for majority carriers, $A^{*}$ is Richardson's constant, $\phi_{\mathrm{b}}$ is the Schottky barrier height, $\mathrm{k}$ is Boltzmann's constant, $\mathrm{T}$ is the temperature, $q$ is the elementary charge and $V$ is the applied voltage. The contribution of minority carrier current which is governed by diffusion at low injection is generally considered negligible in conventional Schottky diodes. For p-type Schottky diodes with uniformly doped substrate, the minority (electron) diffusion current density can be expressed as [1]:

$$
\mathrm{J}_{\mathrm{D}}=\frac{\mathrm{q} \mathrm{D}_{\mathrm{n}} n_{\mathrm{i}}^{2}}{\mathrm{~L}_{\mathrm{n}} \mathrm{N}_{\mathrm{a}}}\left[\exp \left(\frac{\mathrm{qV}}{\mathrm{kT}}\right)-1\right],
$$

where $D_{n}$ and $L_{n}$ is the diffusion constant and diffusion length of the electron in substrate bulk, $\mathrm{N}_{\mathrm{a}}$ is the active acceptor doping concentration of the substrate and $n_{i}$ is the intrinsic carrier concentration of the semiconductor. For situations where $\phi_{b} \ll E_{g}$, where $E_{g}$ is the semiconductor bandgap, the diffusion current $\left(\propto \exp \left(-E_{g}\right)\right)$ is generally much lower than the TE current $\left(\propto \exp \left(-\phi_{\mathrm{b}}\right)\right)$ and therefore can be safely ignored as done conventionally.

However, as $\phi_{\mathrm{b}}$ is raised to higher values, TE is suppressed while the diffusion current remains unaffected. For a sufficiently high $\phi_{\mathrm{b}}$, the diffusion current can be comparable (or even higher) to the TE current. In 
addition, in a high- $\phi_{\mathrm{b}}$ diode, excessive band-bending near the MS interface will result in charge carrier inversion. The induced inversion charge will then act as a source of minority carriers for sustaining the diffusion current which otherwise could reach injection limitation in case of a low- $\phi_{\mathrm{b}}$ diode. These two important phenomena which occur together as a result of a high- $\phi_{\mathrm{b}}$ at the MS interface i.e. suppression of TE current and the sufficient supply of minority carriers transform an otherwise unipolar Schottky device into a (bipolar) p-n junction type device even at low injection.

An analytical model and the conditions for obtaining a sufficiently high$\phi_{\mathrm{b}}$ leading to strong inversion (thus possible bipolar behavior) [55] at a 1-D Schottky electrode were derived before in the chapter 2 [46]. The model indicates that for a suitable metal work function $\left(\phi_{\mathrm{m}}\right)$, that governs $\phi_{\mathrm{b}}$, a sheet of charge carrier of polarity opposite to that of the substrate doping is induced near the MS interface. For a p-type semiconductor, the inversion layer is induced or its effects becomes important provided that [46, 55],

$$
q \phi_{b p}>E_{g}-k T \ln \frac{N_{c}}{N_{a}},
$$

where $\phi_{\mathrm{bp}}$ is the barrier height for holes, $\mathrm{N}_{\mathrm{c}}$ is conduction band effective density of states and $\mathrm{N}_{\mathrm{a}}$ is the acceptor doping density. Eq. (4.1) is equivalent of stating that the induced peak inversion charge (electrons) density $\left(n_{0}=N_{c} \exp \left(-q\left(E_{g}-\phi_{b p}\right) / k T\right)\right.$ is more than the background doping density $\left(\mathrm{N}_{\mathrm{a}}\right)$ of the substrate.

In the past, the two-carrier approach for MS junctions was adopted before by Schwarz and Walsh [136] (refer to appendix B) in connection with their Ge based surface barrier transistor (SBT) device as early as in 1953. The SBT [54] was an interesting demonstration of potential bipolar device technology solely based on MS junctions without any chemical doping. The concept however did not attract much attention after the advent of planar processes and advancement of chemical doping technology ${ }^{1}$.

Scharfetter [137] also investigated the minority carrier injection in Schottky diodes. He concluded that under moderate to high forward bias, Schottky diodes show bipolar behavior where the injection ratio, $\gamma$ (i.e. the ratio of minority carrier current to total current) rises linearly with the applied bias. This was attributed to the drift current of minority carriers which becomes important at higher biases. In this work, Scharfetter [137] did not specifically consider the case of a high- $\phi_{\mathrm{b}}$ diode where the diffusion current could have been important resulting in a high $\gamma$ even at low biases. Moreover, the contact limitations i.e. its ability to supply the required minority carriers was not taken into account in his formulations of current equations which would have otherwise restricted the continuous linear rise of $\gamma$ with increasing forward bias [55] to some maximum value for a given $\phi_{\mathrm{b}}$. Other reports in the literature [138-140] pertaining to minority carrier injection in Schottky diodes have also been more focused on the

\footnotetext{
${ }^{1}$ The SBT will be discussed in more detail in chapter 4 .
} 
high injection regime where the minority drift component becomes important, while the diffusion component at low injection was found to be negligible in conventional Schottky diodes.

A more detailed theoretical analysis on the minority carrier injection in Schottky diodes was reported by Green and Shewchun [55] by applying a combined TE-diffusion theory [141] to account for both majority and minority carrier current flows. They predicted that minority carrier effects could be of considerable importance in case of high- $\phi_{\mathrm{b}}$ diodes even at low injection. Also the effect of high injection and the contact limitations on $\gamma$ was discussed. The work was further extended by Elfsten and Tove [142].

High- $\phi_{\mathrm{b}}$ MS contacts with a considerable minority injection, also referred to as bipolar-mode Schottky contacts [143] could be interesting for power devices to balance the trade-off between the forward voltage drop and breakdown voltage in epitaxial Schottky diodes. Recently, TCAD simulation studies on such 1-D Schottky junctions have been reported for a bipolar transistor [95] and a tunnel diode [96]. Such junctions can also be used for realizing light emitting devices without the need for chemically doped p-n junctions [144]. Moreover this approach of electrostatically forming a localized p-type or n-type region could also be relevant for future CMOS ultra-thin body (UTB) devices where conventional doping is a challenge.

In this chapter, the work of Green and Shewchun [55] on minority carrier injection in Schottky diodes at low injection is further extended with the viewpoint of utilizing this effect for bipolar device applications. The extensive TCAD device simulations are reported to better understand the charge transport mechanisms in different $\phi_{\mathrm{b}} \mathrm{Si}$ diodes, taking into account the effect of the device geometry on current spreading and series resistance. In addition, minority carrier effects in moderately high- $\phi_{\mathrm{b}} \mathrm{Al} / \mathrm{p}-$ Si Schottky diodes are experimentally investigated and compared with TCAD simulations. The experimental findings on an extremely high- $\phi_{\mathrm{b}}$ $\mathrm{Pd} / \mathrm{MoO}_{\mathrm{x}} / \mathrm{n}-\mathrm{Si}$ diodes will be discussed in the next chapter. Furthermore, the technological applications of high- $\phi_{\mathrm{b}}$ Schottky junctions are discussed along with some practical considerations before concluding the findings of this chapter.

\subsection{Device Physics and TCAD Simulation}

\subsubsection{Simulation parameters and models}

Fig. 3.1(a) shows the test structure that was investigated via TCAD simulations [98]. A p-type Si substrate with thickness $t_{S i}$ of $525 \mu \mathrm{m}$ and a doping level $\mathrm{N}_{\mathrm{a}}$ of $10^{15} \mathrm{~cm}^{-3}$ was used. For such doping, a $\phi_{\mathrm{m}}<4.34 \mathrm{eV}$ will result in surface inversion as derived in chapter 2 [46] and possibly bipolar type operation, while $\phi_{\mathrm{m}} \geqslant 4.6 \mathrm{eV}$ will result in a typical Schottky type operation (i.e. unipolar majority carrier transport). Therefore metal work functions 


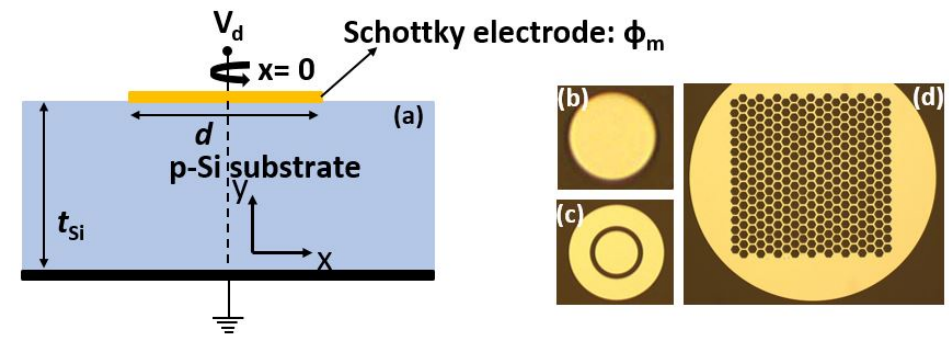

Figure 3.1: (a) Schematic cross-section of the experimental and TCAD simulated device. Optical micrographs (not to the same scale) of fabricated devices: (b) circular electrode with diameter $d=100 \mu \mathrm{m}$, (c) ring geometry as used for the 2-diode test method and (d) hexagonal openings (side $a=10 \mu \mathrm{m}$ ) in the circular electrode $(d=550 \mu \mathrm{m})$ used for infrared light emission measurements.

between $4.2 \mathrm{eV}$ (bipolar operation) and $4.6 \mathrm{eV}$ (Schottky) were adopted for our simulation study. For computation efficiency, a 2-D Delaunay mesh with triangular elements was used. The minimum mesh spacing near the MS interface in the center of the device was $\sim 7 \mu \mathrm{m}$ in the $x$-direction and $\sim 7 \mathrm{~nm}$ in the $\mathrm{y}$-direction. Also, for comparing with experiments and taking minority current spreading into account, a cylindrical symmetry was incorporated around the $y$-axis to solve the Poisson, current and continuity equations. Fermi-Dirac statistics along with the Philips unified mobility model [145] were used. For recombination, doping and temperature dependent Shockley-Read-Hall (SRH: $\tau_{\mathrm{nmax}}=10^{-5} \mathrm{~s}, \tau_{\mathrm{pmax}}=3 \times 10^{-6} \mathrm{~s}$ ) and Auger models [98] were included. Schottky contacts with varying $\phi_{\mathrm{m}}$, however with a fixed (default) surface recombination velocity $\left(S_{n}=2.5 \times 10^{6} \mathrm{~cm} / \mathrm{s}\right.$ and $S_{p}=1.9 \times 10^{6} \mathrm{~cm} / \mathrm{s}$ [98]), were used as a top electrode for all simulations unless otherwise specified. For the bottom electrode, neutral contact, i.e. a one having space charge neutrality and thermal equilibrium at the MS interface, was assumed. The effect of boundary conditions at the bottom electrode on the minority carrier injection is discussed separately in the section 3.2.4. Moreover, ideal interfaces (following the Schottky-Mott relation [127]) were assumed initially for the conceptual understanding of the device operation. Then, the effect of interface traps is discussed.

\subsubsection{Charge transport mechanisms}

Fig 3.2 shows the simulated band diagram and charge carrier profile, both in equilibrium condition, for two different $\phi_{\mathrm{m}}$. The use of $\phi_{\mathrm{m}}=4.6 \mathrm{eV}$ results in a typical Schottky condition with a depletion region at the interface and $\phi_{\mathrm{b}}$ of $0.58 \mathrm{~V}$. However $\phi_{\mathrm{m}}=4.2 \mathrm{eV}$ results in excessive band-bending at the interface with $\phi_{\mathrm{b}}$ of $0.98 \mathrm{~V}$. In this case, the Fermi level at the interface is close to the conduction band, implying surface inversion with a peak induced electron concentration, $\mathrm{n}_{0}$, of $\sim 10^{17} \mathrm{~cm}^{-3}$.

Fig. 3.3 shows simulated I $-V$ characteristics of such Si p-type Schottky diodes for three different $\phi_{\mathrm{b}}\left(\phi_{\mathrm{m}}\right)$. The conduction for the low $\phi_{\mathrm{b}}=0.58$ 
(a)

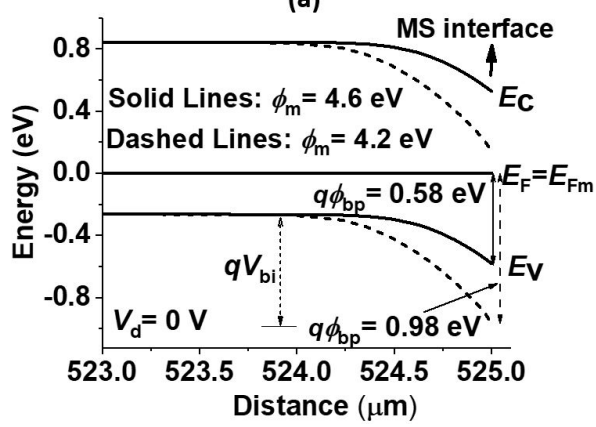

(b)

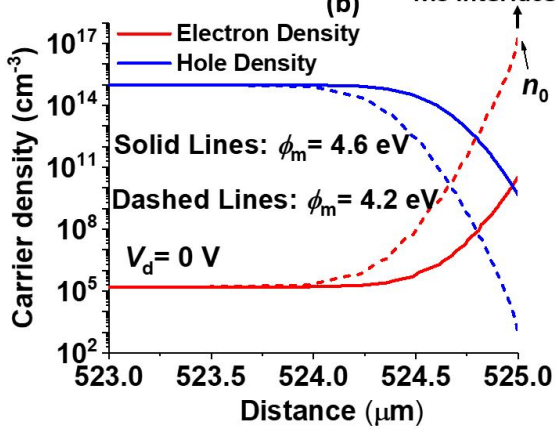

Figure 3.2: Simulated band-diagrams (left) and charge carrier profiles (right) for a Schottky diode with a metal work function $\phi_{\mathrm{m}}=4.6 \mathrm{eV}\left(\phi_{\mathrm{b}}=0.58 \mathrm{~V}\right)$ and $\phi_{\mathrm{m}}=4.2$ $\mathrm{eV}\left(\phi_{\mathrm{b}}=0.98 \mathrm{~V}\right)$ at thermal equilibrium. The origin of the $\mathrm{x}$-axis (Distance) is located at the bottom of the substrate. $V_{b i}$ is the built-in voltage of the diode.

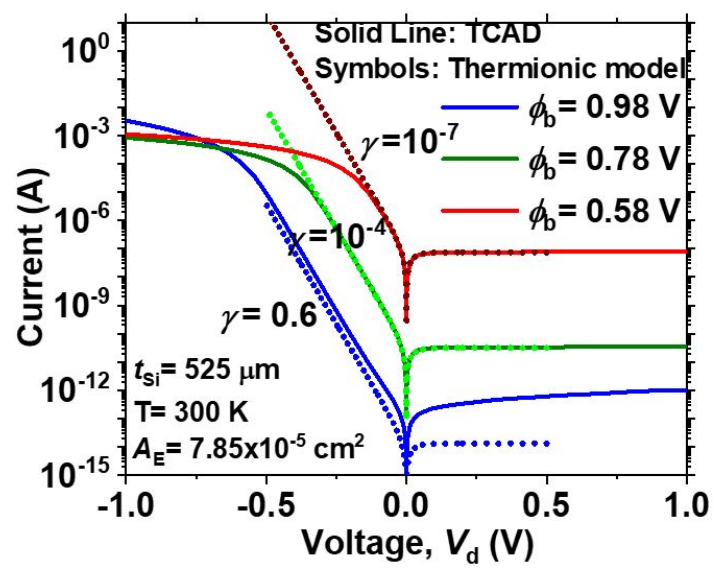

Figure 3.3: Simulated I - V characteristics of Schottky diodes for different $\phi_{\mathrm{b}}\left(\phi_{\mathrm{m}}\right)$ $\gamma$ is calculated at $-0.2 \mathrm{~V}$ (forward bias). For the TE model, $A^{*}=45 \mathrm{Acm}^{-2} \mathrm{~K}^{-2}[98]$ was used for p-type Si.

$\mathrm{V}\left(\phi_{\mathrm{m}}=4.6 \mathrm{eV}\right)$ is solely governed by TE of majority carriers which is much higher than the minority diffusion current as evident from the excellent agreement of TCAD (both forward and reverse current), with the analytical TE I - V model (Eq. (3.1)) [1]. For moderate $\phi_{\mathrm{b}}=0.78 \mathrm{~V}\left(\phi_{\mathrm{m}}=4.4 \mathrm{eV}\right)$, the majority hole current via TE is still dominant. However, a clear deviation from the TE theory is observed for the high $\phi_{\mathrm{b}}=0.98 \mathrm{~V}\left(\phi_{\mathrm{m}}=4.2 \mathrm{eV}\right)$. The TE theory only accounts for the hole current component. The extra current component is attributed to the contribution of electron (minority carrier) diffusion. In this case, with $\phi_{\mathrm{b}}$ as high as $0.98 \mathrm{~V}$, the TE hole current $\left(\mathrm{I}_{\mathrm{h}}\right)$ is sufficiently suppressed that it become comparable to the electron diffusion current $\left(\mathrm{I}_{\mathrm{e}}\right)$. The significant contribution of minority current, $\mathrm{I}_{\mathrm{e}}$, to the total current $\left(\mathrm{I}=\mathrm{I}_{\mathrm{e}}+\mathrm{I}_{\mathrm{h}}\right)$ can be noted from a high minority carrier injection ratio $\gamma\left(\mathrm{I}_{\mathrm{e}} / \mathrm{I}\right)[1]$ in Fig. 3.3. The higher reverse leakage current 


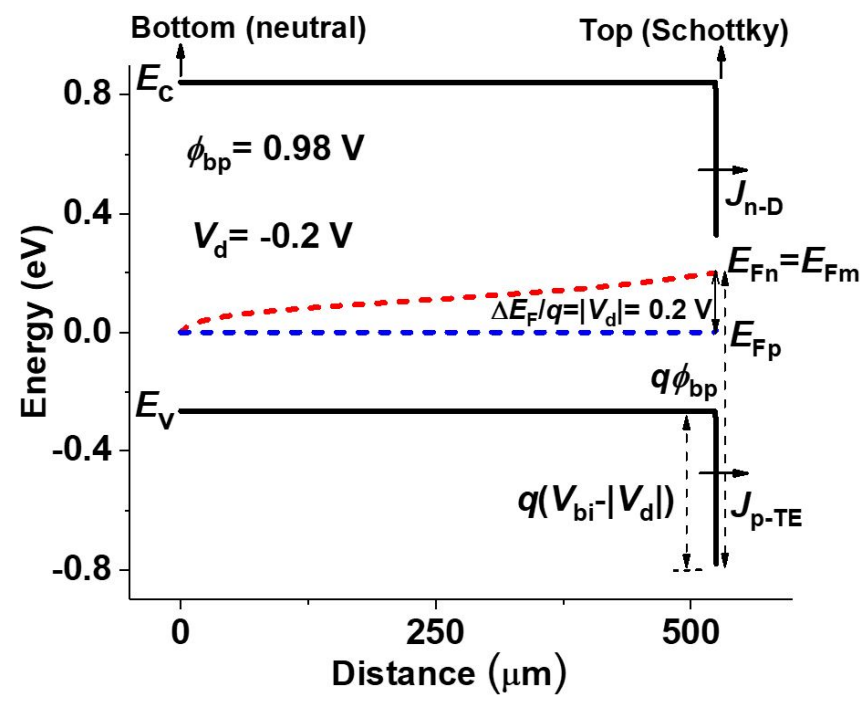

Figure 3.4: Simulated forward bias (at $\mathrm{V}_{\mathrm{d}}=-0.2 \mathrm{~V}$ ) band-diagram of the Schottky diode with $\phi_{\mathrm{b}}=0.98 \mathrm{~V}$.

than predicted by the TE model in case of the high- $\phi_{\mathrm{b}}$ diode is attributed to generation current (see also Fig. 3.5(a)). The observed bipolar conduction in the high- $\phi_{\mathrm{b}}$ diode with a significant $\gamma$ at low injection is counterintuitive to the prevalent perception where Schottky barrier diodes are considered to be majority carrier unipolar devices $[1,146]$.

The forward bias band diagram of a Schottky diode (see Fig. 3.4) can further be used to gain more insight into the prevalent charge transport mechanism at low injection. The presence of minority diffusion current in a Schottky diode can be ascertain from the slope of the electron quasiFermi level $\left(\mathrm{E}_{\mathrm{Fn}}\right)$ in Fig. 3.4. The hole quasi-Fermi level $\left(\mathrm{E}_{\mathrm{Fp}}\right)$ in turn remains flat across the whole device confirming TE as the dominant transport mechanism for the majority carriers [1]. Further, the peak induced electron concentration, $n_{0}$, which is determined by the position of $E_{F n}$ with respect to the $E_{c}$ at the MS Schottky interface, remains fixed to its thermal equilibrium value and doesn't change with the applied bias.

Fig. 3.5(a) shows the electron current $\left(\mathrm{I}_{\mathrm{e}}\right)$ components of the simulated $\mathrm{I}-\mathrm{V}$ curves (Fig. 3.3) for varying $\phi_{\mathrm{b}}$. The diffusion component $\mathrm{I}_{\mathrm{e}}$, being governed by the Gummel number of the substrate $\left(G_{b}=\int_{0}^{t_{5 i}} N_{a}(x) d x\right)$ [147], remains unchanged with varying $\phi_{\mathrm{b}}$ in the exponential region of the $\mathrm{I}-\mathrm{V}$ curves. However, $\gamma$ (see inset Fig. 3.5(a)) varies with $\phi_{\mathrm{b}}$ because the latter affects $I_{h}$. Therefore, $I_{e}$ can be neglected for a low- $\phi_{b}$ (low $\gamma$ ) diode but cannot be ignored for a high- $\phi_{\mathrm{b}}$ (high $\gamma$ ). It is also commonly known that the series resistance of a Schottky diode is higher than that of a p-n juction diode [146] because of the poor supply of minority carriers, (see Fig. 3.5(b)). For $\phi_{\mathrm{b}}=0.58 \mathrm{~V}$ and $0.78 \mathrm{~V}$, the minority carrier concentration is still below $\mathrm{N}_{\mathrm{a}}$ and therefore doesn't affect the overall series resistance 
(a) electrode. current increases with $\phi_{\mathrm{b}}$. (b)

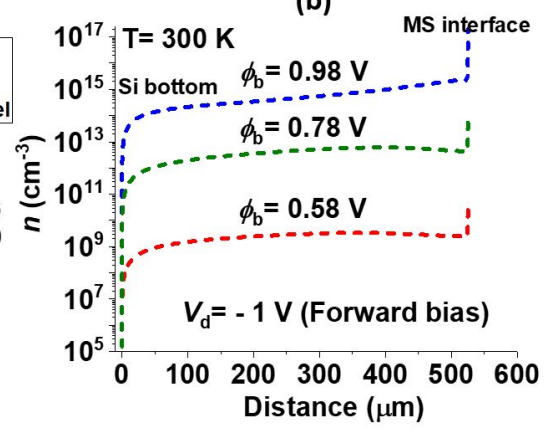

Figure 3.5: (a) Electron current components $\left(\mathrm{I}_{\mathrm{e}}\right)$ of the simulated $\mathrm{I}-\mathrm{V}$ characteristics of Fig. 3.3 at different $\phi_{b}$ (inset: $I_{e}$ and $I_{h}$ for varying $\phi_{b}$ at a fixed forward bias $V_{d}=-0.2 \mathrm{~V}$ ), (b) Simulated electron (minority carrier) profile across the device at $V_{d}=-1 \mathrm{~V}$ forward bias for different $\phi_{\mathrm{b}}$. Note that the peak electron concentration at the MS interface is not affected by the bias. $I_{e}$ and $I_{h}$ are probed at the top Schottky

much. However in case of an extreme $\phi_{\mathrm{b}}=0.98 \mathrm{~V}$, the minority carrier concentration is comparable to $\mathrm{N}_{\mathrm{a}}$ which lowers the series resistance. The effect of $\phi_{\mathrm{b}}$ on the series resistance can be observed from the I $-\mathrm{V}$ plots in Fig. 3.3 and Fig. 3.5(a) at higher biases $\left(V_{d}=-1 V\right.$, forward $)$ where the

Interestingly, such high- $\phi_{\mathrm{b}}$ diodes $\left(\phi_{\mathrm{b}}=0.982 \mathrm{~V}\right.$ for $\left.\mathrm{IrSi} / \mathrm{Si}\right)$ can be realized experimentally, as reported by Wittmer [148, 149], using silicided contacts on bulk Si. However, contrary to our simulation, the author concluded that the measured current fully obeys a single carrier TE theory in the range $200 \mathrm{~K}-300 \mathrm{~K}$ and did not consider the possibility of (minority) diffusion. A possible reason for this could have been the use of a Si substrate with relatively higher Gummel number which actually diminishes the contribution of the diffusion component as discussed further in this chapter. Also, measurements beyond $300 \mathrm{~K}$ were not reported where diffusion would have become relatively more noticeable. Moreover, a possibly high surface recombination velocity at the Schottky contact could also enhance the TE current and thereby degrading the $\gamma$, which otherwise would have been expected to be relatively high for such $\phi_{\mathrm{b}}$.

\subsubsection{Role of inversion layer: Injection limited regime}

The contact limitations upon injecting minority carriers at high injection has been highlighted before by Green and Shewchun [55]. In order to understand the role of inversion layer at the contact in limiting the minority current at moderate to high forward biases, its important to first minimize the effect of series resistance while keeping the minority (electron) current the same. For this purpose, a Si Schottky diode structure with a thin, $5 \mu \mathrm{m}$, p-type $\left(10^{15} \mathrm{~cm}^{-3}\right)$ layer on top of a $\mathrm{p}^{+}\left(10^{19} \mathrm{~cm}^{-3}\right)$ substrate is considered. 


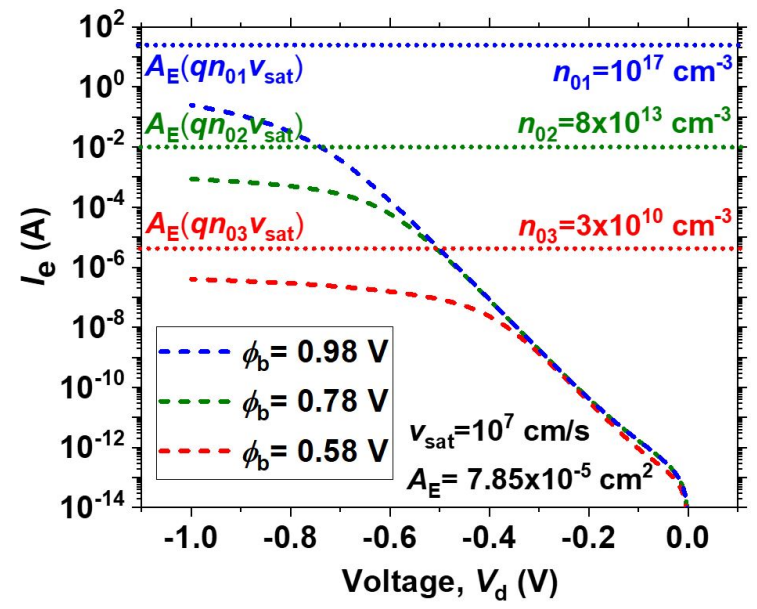

Figure 3.6: (a) Minority (electron) current components $\left(\mathrm{I}_{\mathrm{e}}\right)$ of the simulated $\mathrm{I}-\mathrm{V}$ characteristics of the $\mathrm{pp}^{+}$Schottky diode with the same effective Gummel number as that of bulk p-type substrate in Fig. 3.3 but with a lower series resistance. The dotted horizontal lines indicate the upper limit of the minority current that the Schottky contact with the given $\phi_{\mathrm{b}}$ can supply.

The thickness of the $\mathrm{p}^{+}$substrate was adjusted such that the (effective) Gummel number of this structure is the same as that of the earlier used bulk, $525 \mu \mathrm{m}$ thick, p-type $\left(10^{15} \mathrm{~cm}^{-3}\right)$ substrate. From hereafter, this type of device will be referred as a $\mathrm{pp}^{+}$Schottky diode.

Fig. 3.6 shows the electron current $\left(\mathrm{I}_{\mathrm{e}}\right)$ against the voltage for varying $\phi_{\mathrm{b}}$ in case of the $\mathrm{pp}^{+}$Schottky diode with a lower series resistance. Clearly, the minority current which is governed by diffusion at lower biases becomes injection limited at higher biases. The upper limit of the minority (electron) current that the contact with given $\phi_{\mathrm{b}}$ can supply is approximated as $\mathrm{I}_{\mathrm{e}} \sim A_{\mathrm{E}} q n_{0} v_{\text {sat }}$. Here $n_{0}$ refers to the peak induced electron concentration at the MS interface for given $\phi_{\mathrm{b}}, A_{\mathrm{E}}$ is the area of top electrode and $v_{\mathrm{sat}}$ is the saturation velocity for electrons, $\sim 10^{7} \mathrm{~cm} / \mathrm{s}$. Therefore, it can be concluded that the inversion layer and consequently a high- $\phi_{\mathrm{b}}$ is important in sustaining a minority diffusion current at moderate to high biases for any bipolar device application.

A similar argument holds true even for majority carriers. For a neutral type back contact, the maximum value of majority current is then limited by the doping density of the substrate and is given by $I_{e}=A_{E} q N_{a} v_{\text {sat }}$. Beyond this limit, the majority current will then become bulk limited and will be no longer governed by TE. In practice, for bulk substrates, such effects are masked by the series resistance effect which comes into play before the injection limited regime for minority carriers or bulk limited regime for majority carriers sets in. However, this could be important for thinner substrates or high mobility semiconductors where the series resistance is lower. 

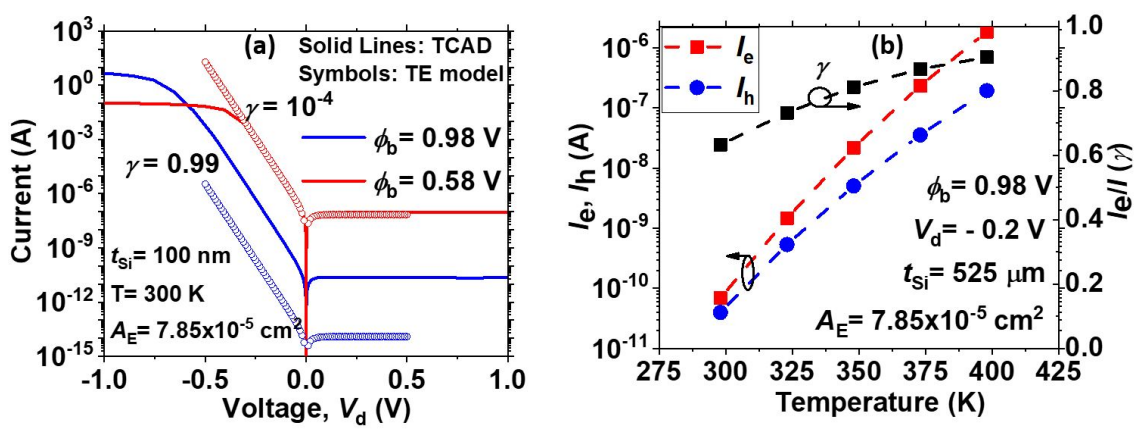

Figure 3.7: Simulated $I-V$ characteristics of a Schottky diode (a) with $t_{\mathrm{Si}}=100 \mathrm{~nm}$ for both high- $\phi_{\mathrm{b}}$ and low- $\phi_{\mathrm{b}}$ diodes. $\gamma$ is calculated at $-0.2 \mathrm{~V}$ (forward bias) (b) $\mathrm{I}_{\mathrm{e}}$ and $I_{h}$ against the temperature for the high- $\phi_{b}$ diode $\left(V_{d}=-0.2 V, t_{S i}=525 \mu \mathrm{m}\right)$.

\subsubsection{Tuning the minority diffusion component}

\section{Substrate thickness reduction}

The diffusion current can be further increased by reducing the Gummel number of the substrate. By reducing $\mathrm{t}_{\mathrm{Si}}$ from $525 \mu \mathrm{m}$ to $100 \mathrm{~nm}, \gamma$ increases from 0.6 to 0.99 for $\phi_{b}=0.98 \mathrm{~V}$ as shown in Fig. 3.7(a). Here a larger deviation from the TE model is observed implying that the current is largely diffusion dominated. However varying $t_{S i}$ doesn't affect the TE majority current and therefore the current for a device with $\phi_{\mathrm{b}}=0.58 \mathrm{~V}$ is still following the TE model. The pronounced effect of the diffusion current for smaller substrate thicknesses in high- $\phi_{\mathrm{b}}$ diodes could be relevant for bipolar applications in advanced UTB devices.

\section{Elevated temperatures}

Also the temperature dependence of diffusion is larger than that of TE because of the relatively high potential barrier in case of the former [1]. Therefore, for the high- $\phi_{\mathrm{b}}$ diode, $\gamma$ increases with the temperature as shown in Fig. 3.7(b). Such a rise in the diffusion current at higher temperatures was also observed experimentally in our Schottky diodes as discussed in section 3.3.3.

\section{Role of bottom contact}

For the simulation study performed so far in this chapter, the substrate bottom contact was assumed to be neutral in nature. However, it has been shown before $[137,150]$ that the boundary conditions at the bottom contact also affect the minority injection at the top contact. The effect of the bottom contact becomes important when the (minority) diffusion length is larger than or comparable to the substrate thickness. In order to illustrate this, TCAD simulations were performed using a thin Si substrate $\left(t_{\mathrm{Si}}=5\right.$ 

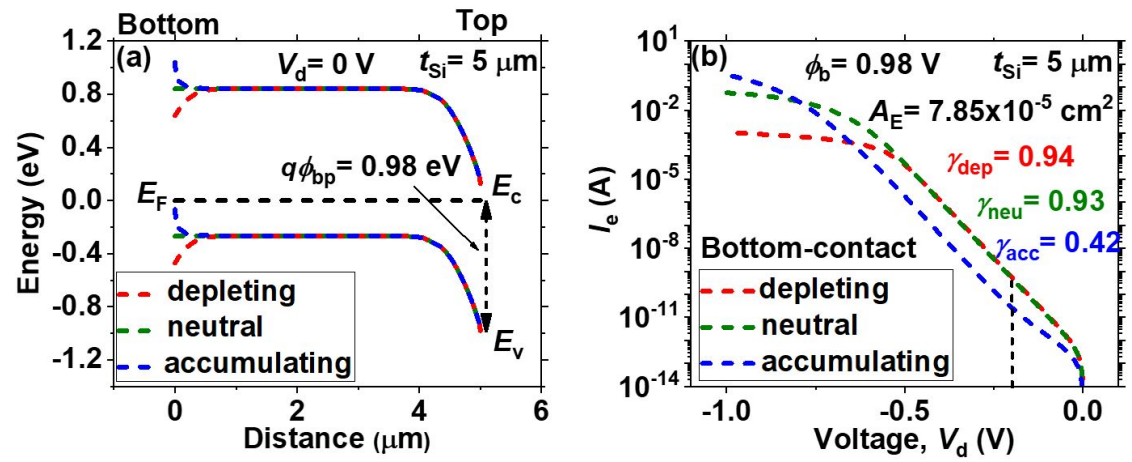

Figure 3.8: Simulated (a) energy band diagram at thermal equilibrium and (b) minority (electron) current at top Schottky contact (with fixed $\phi_{\mathrm{b}}=0.98 \mathrm{~V}$ ) for varying boundary conditions at the bottom substrate contact from depleting to accumulating. $t_{\mathrm{Si}_{\mathrm{i}}}=5 \mu \mathrm{m}$ was used for this simulation.

$\mu \mathrm{m})$ for three different types of bottom contact, i.e. depleting, neutral and accumulating. The $\phi_{\mathrm{b}}$ at the top Schottky contact was fixed to $0.98 \mathrm{~V}$.

Fig. 3.8(a) shows the energy band diagram for the three different conditions at the bottom contact. In case of the depleting contact, downwards band bending at the bottom contact favors the electron extraction and, as a result, the electron current is enhanced at the top contact as can be seen in Fig. 3.8(b). For the neutral contact, the electron current is the same as that of the depleting contact but with a lower series resistance. However, in case of the accumulating contact, the upward band bending act as a barrier for the electrons extraction at the bottom contact and, as a result, the electron current is suppressed at the top contact. The accumulating type of contact, though preferred for their lower resistance, adversely affects the minority injection which could be a concern if a high $\gamma$ is desired. The majority (hole) current (not shown) at the top contact remains the same for all three cases except for the series resistance part which shows the same trend as depicted in Fig. 3.8(b) at higher bias voltages.

\subsubsection{Small-size Schottky contacts}

Other than tuning the minority diffusion component alone by varying the substrate Gummel number, the injection ratio $\gamma$ can be increased further by suppressing the majority current component. One approach of achieving this is by reducing the area of the Schottky contact to which the majority carriers TE current is directly proportional. The minority current on the other hand spreads away from the contact into the substrate (discussed further in section 3.3.3) and therefore depends both on area as well as the perimeter of the contact. For a very small-size contact (sub $100 \mathrm{~nm}$ ) with a large minority current spreading, a high $\gamma$ is therefore expected.

To study this effect, the area of the top Schottky contact was greatly reduced by decreasing its radius (r) from $50 \mu \mathrm{m}$ to $50 \mathrm{~nm}$ and the relative 

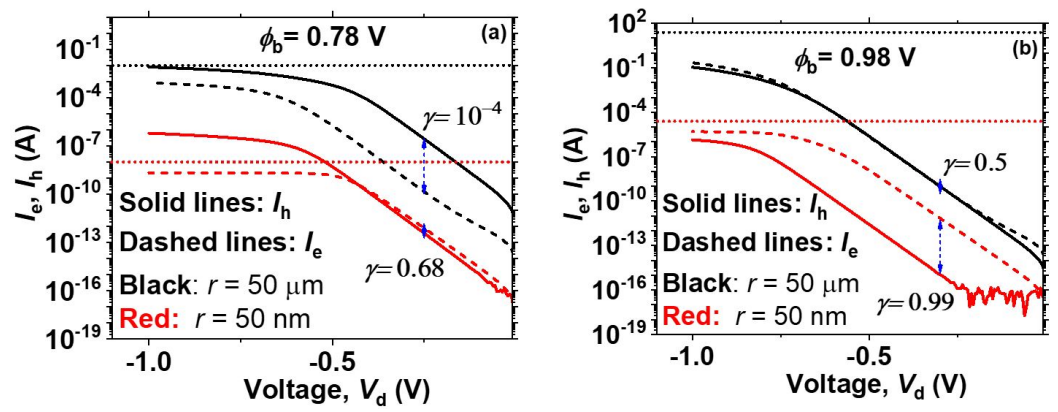

Figure 3.9: Simulated electron $\left(\mathrm{I}_{\mathrm{e}}\right)$ and hole $\left(\mathrm{I}_{\mathrm{h}}\right)$ current components for two different radii $(r)$ of the top Schottky contact with (a) $\phi_{b}=0.78 \mathrm{~V}$ and (b) $\phi_{b}=0.98 \mathrm{~V}$. The dotted horizontal lines indicate the upper limit of the minority current that the Schottky contact with a given $\phi_{\mathrm{b}}$ can supply.

magnitudes of $I_{e}$ and $I_{h}$ were then compared in both cases. Note that for this simulation, in order to minimize the series resistance effect, the $\mathrm{pp}^{+}$ Schottky diode structure was used as introduced before with the same Gummel number as that of the bulk substrate.

For $\phi_{\mathrm{b}}=0.78 \mathrm{~V}$, see Fig. 3.9(a), the $\gamma$ in the low bias region is significantly higher for the $50 \mathrm{~nm}$ contact as compared to that of $50 \mu \mathrm{m}$ where it is negligible. For $\phi_{\mathrm{b}}=0.98 \mathrm{~V}, \gamma$ which is already high even for the $50 \mu \mathrm{m}$ contact, is further improved when the contact radius is reduced to $50 \mathrm{~nm}$. The injection ratio however gradually drops at higher forward biases as the minority current reaches its injection limitation for a given barrier height. The effect is more pronounced for a lower $\phi_{\mathrm{b}}$ due to the poor minority carriers supply to sustain the diffusion current level at higher biases. The situation at higher biases is relatively better for a larger $\phi_{b}$ with its improved minority carrier supply.

This idea of achieving a high injection ratio even with a nominally high $\phi_{\mathrm{b}}$ using a sub $100 \mathrm{~nm}$ size contact could be interesting for realizing bipolar devices. The upper limit of the operating voltage (current) is however restricted by $\phi_{\mathrm{b}}$ of the Schottky contact.

Further, the small-size Schottky contact is also a better light emitter than its big-size counterpart. This can be analyzed from Fig. 3.10(a) where at low biases the effective minority (electron) current density, $\mathrm{Je}_{\mathrm{e}}$, for the $50 \mathrm{~nm}$ contact is shown to be several orders $(\sim 3)$ in magnitude higher than that of the $50 \mu \mathrm{m}$ contact due to a higher perimeter contribution in the former than the latter. Now in Schottky diodes, the light emission (or radiative recombination) is governed by the injected minority current. Therefore, it is expected that the light emission per unit area from the 50 $\mathrm{nm}$ contact is also higher than that of $50 \mu \mathrm{m}$ contact by similar orders in magnitude. This is manifested by Fig. 3.10(b) where indeed a significantly higher (also by $\sim 3$ orders in magnitude) per unit area light output from the former at low biases can be deduced from the plot of integrated radiative recombination rate $\left(\mathrm{R}_{\mathrm{i}-\mathrm{rad}}\right)$ per unit area against the applied bias. This 

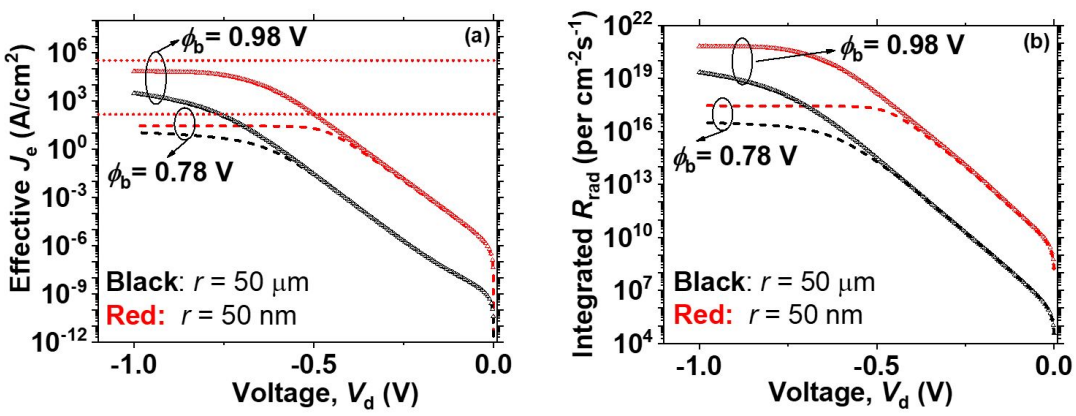

Figure 3.10: Simulated (a) effective minority (electron) current density $\left(\mathrm{J}_{\mathrm{e}}\right)$ and $(\mathrm{b})$ integrated radiative recombination rate $\left(\mathrm{R}_{\mathrm{i}-\mathrm{rad}}\right)$ per unit area for two different radii ( $r$ ) of the top Schottky contact shown for $\phi_{b}=0.78 \mathrm{~V}$ and $\phi_{b}=0.98 \mathrm{~V}$. The dotted horizontal lines indicate the upper limit of the minority current density that the Schottky contact with a given $\phi_{\mathrm{b}}$ can supply.

observation can have important consequences for designing light emitters based on Schottky contacts. Basically, it implies that for a given metal $\left(\phi_{\mathrm{b}}\right)$, the array of $\mathrm{nm}$ size contacts when placed sufficiently apart so as not to affect their individual current spreading into the substrate, will as a whole emit more light compared to a $\mu \mathrm{m}$ size single contact with an effective area equal to that of the whole nm size contact array.

However the difference in $\mathrm{J}_{\mathrm{e}}$ and therefore light emission among different size contacts fades out at higher biases where the current density is then ultimately capped by its maximum injection limit which in turn is governed by $\phi_{\mathrm{b}}$. Again the effect is more pronounced for lower- $\phi_{\mathrm{b}}$ diodes than higher $\phi_{b}$ counterparts as can be noted from Fig. 3.10(a)-(b). Table 3.1 summarizes the TCAD simulation results for various $\phi_{b}$ and device geometries.

Table 3.1: Summary of TCAD simulation results for various $\phi_{\mathrm{b}}$, substrate thicknesses $\left(t_{S i}\right)$, Schottky contact areas $\left(A_{E}\right)$ and temperatures $(T)$ at $V_{d} \sim-0.2 V$. The term "pp ${ }^{+}$here refers to the Schottky diode on $\mathrm{pp}^{+}$substrate with the same Gummel number as the bulk $525 \mu \mathrm{m}$ p-type substrate.

\begin{tabular}{|c|c|c|c|c|c|}
\hline$\phi_{\mathrm{b}}(\mathrm{V})$ & $\mathrm{T}(\mathrm{K})$ & $\mathrm{t}_{\mathrm{Si}}(\mu \mathrm{m})$ & $A_{\mathrm{E}}\left(\mathrm{cm}^{2}\right)$ & $\gamma$ & Dominant mechanism \\
\hline 0.58 & 298 & 525 & $7.85 \times 10^{-5}$ & $10^{-7}$ & $\mathrm{TE}$ \\
\hline 0.58 & 298 & 0.1 & $7.85 \times 10^{-5}$ & $10^{-4}$ & $\mathrm{TE}$ \\
\hline 0.78 & 298 & 525 & $7.85 \times 10^{-5}$ & $10^{-4}$ & $\mathrm{TE}$ \\
\hline 0.98 & 298 & 525 & $7.85 \times 10^{-5}$ & 0.6 & minority diffusion, TE \\
\hline 0.98 & 298 & 0.1 & $7.85 \times 10^{-5}$ & 0.99 & minority diffusion \\
\hline 0.98 & 398 & 525 & $7.85 \times 10^{-5}$ & 0.9 & minority diffusion \\
\hline 0.78 & 298 & $525\left(\mathrm{pp}^{+}\right)$ & $7.85 \times 10^{-11}$ & 0.68 & minority diffusion, TE \\
\hline 0.98 & 298 & $525\left(\mathrm{pp}^{+}\right)$ & $7.85 \times 10^{-11}$ & 0.99 & minority diffusion \\
\hline
\end{tabular}




\subsection{Experimental Results}

\subsubsection{Device Fabrication}

p-Si Schottky diodes were fabricated ${ }^{2}$ using $\mathrm{Al}$ as a top electrode (Fig. 3.1). The choice of $\mathrm{Al}$ is based on its reported low vacuum work function, $\phi_{\mathrm{m}}$, of $4.28 \mathrm{eV}$ which ideally should result in a $\phi_{\mathrm{b}}$ of $0.89 \mathrm{~V}\left(\mathrm{q} \phi_{\mathrm{b}}=\mathrm{E}_{\mathrm{g}}-\phi_{\mathrm{m}}+\chi_{\mathrm{s}}\right)$, where $E_{g}$ is the $S i$ bandgap $(1.12 \mathrm{eV})$ and $\chi_{s}$ is the Si electron affinity (4.05 $\mathrm{eV})$. The substrate back was utilized as the bottom electrode. A (111) p-type $\mathrm{Si}$ substrate with $\mathrm{N}_{\mathrm{a}}$ of $10^{15} \mathrm{~cm}^{-3}$ and with a native oxide was used. $200 \mathrm{~nm} \mathrm{Al}$ was sputtered on the polished surface. The electrodes were then patterned using a standard photolithography process using a positive photoresist. The presence of the thin native oxide between $\mathrm{Al}$ and Si surface avoids chemical inter-diffusion and also decouples the electron states in the Si from the metal $[83,151]$, which might lower the effective $\phi_{\mathrm{b}}$. Annealing the wafer was avoided to prevent alloy formation and to keep the as-deposited metallurgical nature of the junction intact. Note that, from view point of ruggedness and reproducibility this annealing step should not be avoided. As a reference, to obtain a low- $\phi_{\mathrm{b}}$ diode, an $\mathrm{n}-\mathrm{Si} / \mathrm{Al}$ wafer was also fabricated following a similar process.

\subsubsection{Electrical characterization: Barrier height extraction}

Temperature dependent I $-\mathrm{V}$ measurements of the fabricated $\mathrm{Al} / \mathrm{p}-\mathrm{Si}$ Schottky diodes were performed using a Keithley 4200 Semiconductor Characterization System (SCS) with a preamplifier enabling DC measurements with a combined noise level of $\sim 10 \mathrm{fA}$ for an integration time of 100 $\mathrm{ms}$. The measurements were performed under dark conditions and on a temperature controlled chuck.

A moderately high $\phi_{\mathrm{b}}$ of $0.77 \mathrm{~V}$ with an ideality factor $\mathrm{n}$ of 1.09 was extracted from the Richardson plot (see Fig. 3.11(a)) in the exponential region $\left(V_{d}=-0.2 \mathrm{~V}\right)$ of the $I-V$ curves. The extracted $\phi_{b}$ from I $\mathrm{V}(-\mathrm{T})$ measurements is also in agreement with that obtained from $\mathrm{C}-\mathrm{V}$ measurements as shown in Fig. 3.11(c). The relatively low value of the extracted $A^{*}\left(4.06 \mathrm{Acm}^{-2} \mathrm{~K}^{-2}\right)$ as compared to the reported value of 32 $\pm 2 \mathrm{Acm}^{-2} \mathrm{~K}^{-2}[1,152]$ for a $\mathrm{p}$-Si substrate can be partly caused by the inhomogeneity at the interface [153] as a result of the unannealed electrical contacts. Further, the non-ideality of $n \sim 1.09$ could be attributed to the presence of the interfacial layer (native oxide) and image force effect [1]. However, since the effective $\phi_{\mathrm{b}}>0.5 \mathrm{E}_{\mathrm{g}} / \mathrm{q}$, some level of surface inversion is expected in this diode. Similar measurements on the n-Si/Al diode results in a low $\phi_{\mathrm{b}}$ of $0.53 \mathrm{~V}$ as desired for comparison. Note that the sum of $q \phi_{b}$ on p-type and n-type substrate is higher than $E_{g}(1.12 \mathrm{eV})$. This could be attributed to the presence of native oxide and/or Fermi-level pinning due to interface states.

\footnotetext{
${ }^{2}$ The device fabrication part as reported in this chapter was performed by S. Banerjee.
} 

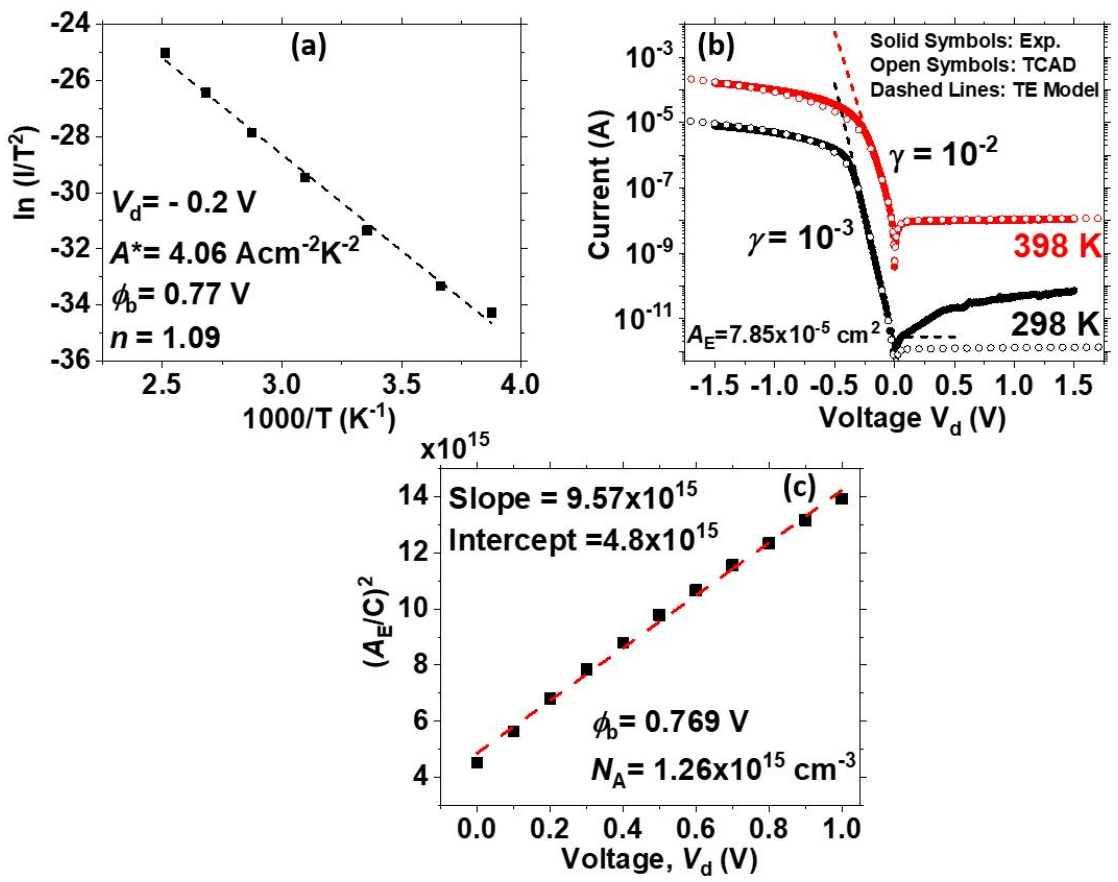

Figure 3.11: (a) Richardson plot of the fabricated $\mathrm{Al} / \mathrm{p}$-Si diode $\left(A_{\mathrm{E}}=7.85 \times 10^{-5} \mathrm{~cm}^{2}\right)$ at $V_{d}=-0.2 \mathrm{~V}$ forward bias. A $\phi_{\mathrm{b}}$ of $0.77 \mathrm{~V}$ was extracted using this measurement. (b) Measured I $-V$ curves at $T=298 \mathrm{~K}$ and $\mathrm{T}=398 \mathrm{~K}$ and their fitting using TCAD simulation and the analytical TE model (Eq. (3.1)) with experimentally extracted values of $\phi_{b}$, Richardson's constant $A^{*}$ and ideality factor $n$. (c) $\phi_{b}$ extraction from $\mathrm{C}-\mathrm{V}$ analysis in reverse bias.

The extracted $\phi_{\mathrm{b}}$ of $0.77 \mathrm{~V}$ was used as input for the TCAD simulation where excellent agreement with the experimental data was obtained in forward bias operation, as shown in Fig. 3.11(b). $\phi_{\mathrm{b}}$ of $0.77 \mathrm{~V}$ results in a downward band bending near the MS interface with a peak induced electron concentration, $\mathrm{n}_{0}$ of $3 \times 10^{13} \mathrm{~cm}^{-3}$. Note that, this induced electron concentration for the moderately high experimental $\phi_{\mathrm{b}}$ of $0.77 \mathrm{~V}$ is much lower than that for the extremely high $\phi_{\mathrm{b}}$ of $0.98 \mathrm{~V}\left(\phi_{\mathrm{m}}=4.2 \mathrm{eV}\right)$ adapted for simulations in Section 3.2. Therefore, less pronounced effects on the conduction are expected. Consequently, in our experimental devices the current is largely dominated by TE majority carriers, i.e. holes, with $\gamma=10^{-3}$. This is further evident as experimental and TCAD I $-V$ characteristics are in agreement with the TE analytical model (Eq. (3.1)). The relative contribution of the electron diffusion though increases at higher temperatures to $\gamma=10^{-2}$. However, $\mathrm{J}$ is still governed by TE. Therefore, in this case it is not straightforward to establish the presence of minority carriers experimentally. 

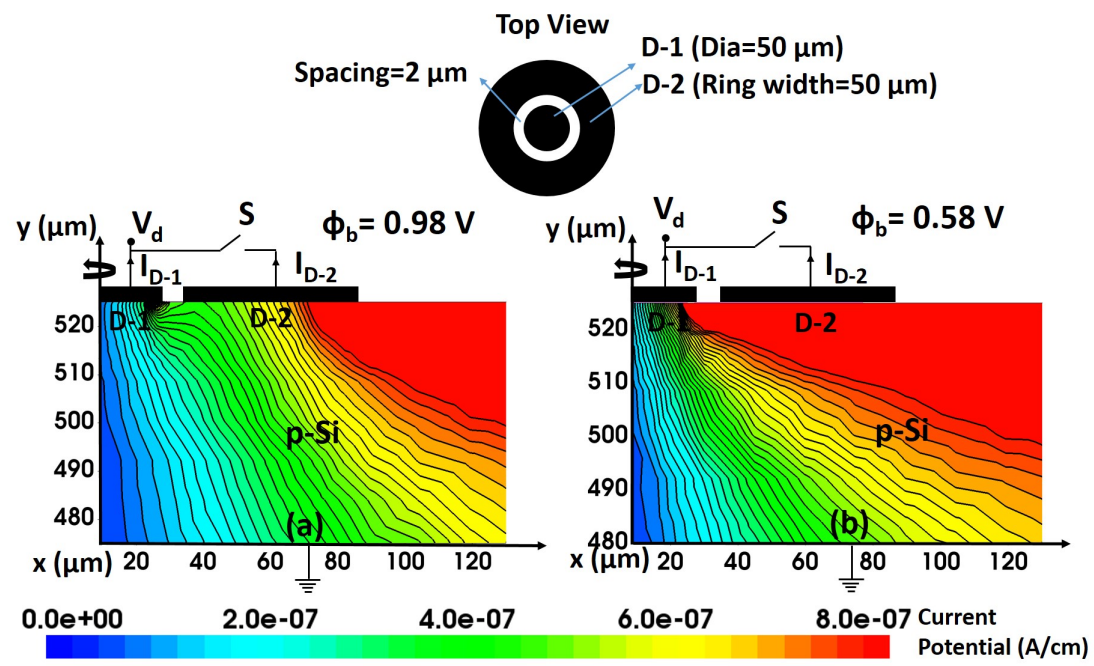

Figure 3.12: Schematic of the 2-diode test method and measurement configuration with TCAD simulated current flow lines. The flow lines indicate the current spreading in the device when only $\mathrm{D}-1$ is biased (forward current bias $=1 \mu \mathrm{A}$ ) for case (a) $\phi_{\mathrm{b}}=0.98 \mathrm{~V}$ and (b) $\phi_{\mathrm{b}}=0.58 \mathrm{~V}$. The current spreading due to minority carrier injection is observed near the contact for the high- $\phi_{b}$ diode, while less current spreading was observed for the low- $\phi_{\mathrm{b}}$ diode. The switch $S$ is in closed position when both $\mathrm{D}-1$ and $\mathrm{D}-2$ are biased.

\subsubsection{2-diode measurements}

To study the influence of minority carrier injection in a high- $\phi_{\mathrm{b}}$ diode, a prior reported 2-diode method $[154,155]$ was adopted. With this technique, it is possible to distinguish the operation of a (Schottky based) shallow p-n like junction diode from that of a conventional Schottky diode at low injection.

The schematic of the 2-diode test method is illustrated in Fig. 3.12 where two similar diodes, $\mathrm{D}-1$ and $\mathrm{D}-2$, are closely spaced $(\sim 2 \mu \mathrm{m})$. Here the current of $D-1$ is measured when it is operated in single mode, and in parallel mode along with $\mathrm{D}-2$. In case of a $\mathrm{p}-\mathrm{n}$ type diode, there will be a significant contribution of minority carrier injection from the contacts (high $\gamma$ ), and as a result the current will spread outwards from the contacts. When $D-1$ is operated in parallel with $D-2$, then in case of $p-n$ type diodes, the current of each diode will be less compared to that in single mode operation due to influence of current spreading from the neighboring diode. This influence can be measured from the differential current level in the exponential part of the I $-\mathrm{V}$ curve.

While in case of a conventional Schottky diode, the minority carrier injection will be insignificant and therefore there will be hardly any current spreading near the contacts. Hence, for conventional Schottky diodes, the current for both modes (i.e. single mode and parallel mode operation) 


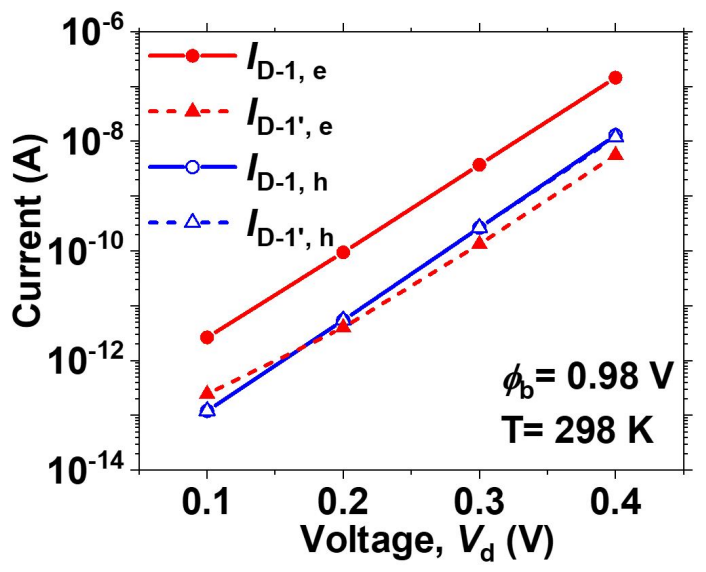

Figure 3.13: Simulated electron $\left(\mathrm{I}_{\mathrm{e}}\right)$ and hole $\left(\mathrm{I}_{\mathrm{h}}\right)$ currents of diode $\mathrm{D}-1$ when operated in single mode $\left(\mathrm{I}_{\mathrm{D}-1}\right)$ and in parallel mode $\left(\mathrm{I}_{\mathrm{D}-1^{\prime}}\right)$ for high $\phi_{\mathrm{b}}(=0.98 \mathrm{~V})$ Schottky diodes. $\mathrm{t}_{\mathrm{Si}}=525 \mu \mathrm{m}$ and $\mathrm{N}_{\mathrm{A}}=10^{15} \mathrm{~cm}^{-3}$.

will be the same as it is governed by majority carrier injection from the substrate and depends only on the area of the diode. Therefore then hardly any influence of the neighboring diode will be noticeable in the exponential part of the I $-\mathrm{V}$ curve.

Fig. 3.13 shows the TCAD simulated electron $\left(\mathrm{I}_{\mathrm{e}}\right)$ and hole $\left(\mathrm{I}_{\mathrm{h}}\right)$ currents in case of high- $\phi_{\mathrm{b}}$ Schottky diodes when diode $\mathrm{D}-1$ is operated in single mode $\left(I_{D-1}\right)$ and in parallel mode $\left(I_{D-1}\right)$ with diode $D-2$. As expected, only the minority (electron) current is affected in parallel mode operation while the majority current remains unaffected. Since in case of a high$\phi_{\mathrm{b}}$ diode, $\gamma$ is high, this influence can be measured in the 2-diode test configuration.

Fig. 3.14(a)-(b) shows the experimental 2-diode characteristics for two different $\phi_{\mathrm{b}}$ diodes. For the $\mathrm{p}-\mathrm{Si} / \mathrm{Al}$ diode with $\phi_{\mathrm{b}}=0.77 \mathrm{~V}$, a slight decrease
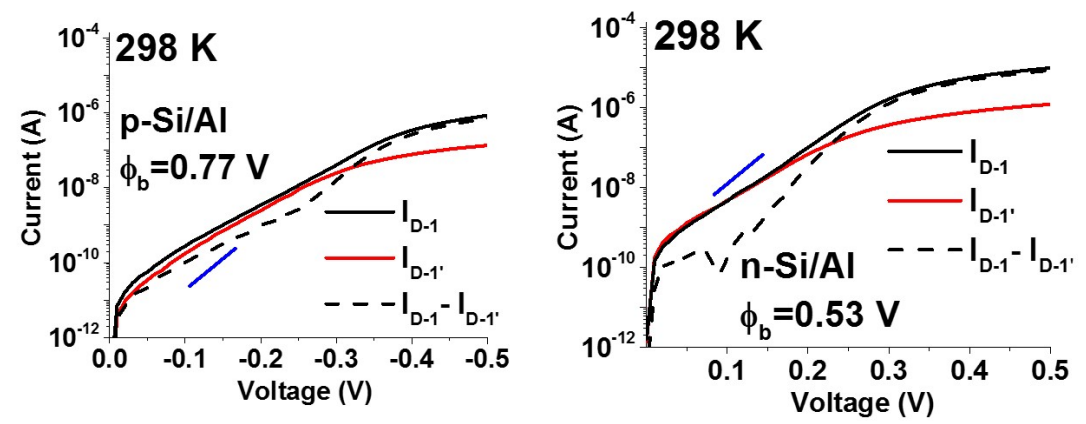

Figure 3.14: I - V curves showing the measured D - 1 current when operated in single mode and parallel mode at $298 \mathrm{~K}$ for (a) $\phi_{\mathrm{b}}=0.77 \mathrm{~V}$ and (b) $\phi_{\mathrm{b}}=0.53 \mathrm{~V}$. The blue solid line indicates an ideality factor $n=1$ for reference. 
in the diode $\mathrm{D}-1$ current $\left(\Delta \mathrm{I}=\mathrm{I}_{\mathrm{D}-1}-\mathrm{I}_{\mathrm{D}-1^{\prime}}\right)$ in the exponential part of the plot when operated in parallel with diode $\mathrm{D}-2$ was observed as shown in Fig. 3.14(a). Here, a current discrepancy $(\Delta \mathrm{I} / \mathrm{I})$ of $37 \%$ was observed in the forward bias exponential region at $V_{d}=-0.1 \mathrm{~V}$. This indicates the influence of minority carrier (electron) injection in the p-type Schottky diode. However, in case of the $\mathrm{n}-\mathrm{Si} / \mathrm{Al}$ diode with a near mid-gap $\phi_{\mathrm{b}}=$ $0.53 \mathrm{~V}$, the influence of the minority carrier injection from the metal side into the semiconductor is negligible and thus the $\mathrm{D}-1$ current remains unaffected as shown in Fig. 3.14(b). Here a current discrepancy $(\Delta \mathrm{I} / \mathrm{I})$ of less than $7 \%$ was observed at $V_{d}=0.1 \mathrm{~V}$. Further, the influence of current spreading increases at higher temperatures where a higher $\Delta \mathrm{I} / \mathrm{I}=51 \%$ was noted at $398 \mathrm{~K}$ for diodes with $\phi_{\mathrm{b}}=0.77 \mathrm{~V}$. This is expected as an increase in temperature increases the diffusion component more than the TE current and will raise $\gamma$, in this case, by one order of magnitude, as observed in our TCAD simulations (Fig. 3.7(b)). The observed lower $\mathrm{I}_{\mathrm{D}-1^{\prime}}$ in the series resistance regime for both diodes in Fig. 3.14(a)-(b) is due to the high resistance encountered during parallel mode operation [154, 155].

\subsubsection{Electroluminescence (EL) measurements}

The light emission in the Schottky diode during its forward bias operation is determined by its injected minority current as discussed in section 3.2.5 (Fig. 3.10) using TCAD simulations. It can be stated that at low bias operation, when the minority current densities in two different $\phi_{\mathrm{b}}$ diodes are the same then the light emission or radiative recombination rates will also identical in two diodes. However, in the high bias regime where the series resistance or injection limitation becomes important, the higher$\phi_{\mathrm{b}}$ diode shows a higher minority current density and hence more light emission compared to that of a lower $\phi_{b}$ diode. Further, for a constant current drive at low bias, the voltage drop across the junction increases with $\phi_{\mathrm{b}}$ and consequently the diffusion current and $p \cdot n$ product (thus light emission) also increases with $\phi_{\mathrm{b}}$ in that regime.

In order to support our TCAD findings, the electroluminescence (EL) from our fabricated Si-Schottky diodes was measured. A cooled InGaAs detector based camera (XEVA-320 from Xenics) with a spectral detection range of $950 \mathrm{~nm}-1700 \mathrm{~nm}$ was used for capturing the EL micrograph using an integration time of $30 \mathrm{~s}$. Low-intensity light emission in the infrared (IR) range was observed from our $\mathrm{p}-\mathrm{Si} / \mathrm{Al}$ diodes $\left(\phi_{\mathrm{b}}=0.77 \mathrm{~V}\right)$ at a constant current drive of $20 \mathrm{~mA}\left(\mathrm{~J} \sim 25 \mathrm{~A} / \mathrm{cm}^{2}\right)$ at room temperature, as shown in Fig. 3.15(a). The light emission occurs via band-to-band recombination of injected minorities (electrons) with background majorities (holes) in the p-type Si substrate. In addition, the emitted light was measured via the short-circuit current ( $\mathrm{I}_{\mathrm{PD}}$ ) of an off-chip Si-photodiode [156] as shown in Fig. 3.15(b). The $\mathrm{I}_{\mathrm{PD}}$ is proportional to the emission intensity which rises steadily with the injected current $\mathrm{I}_{\mathrm{LED}}$ of the high- $\phi_{\mathrm{b}}$ Schottky diode. 


\section{Circular electrode with hexagonal openings}
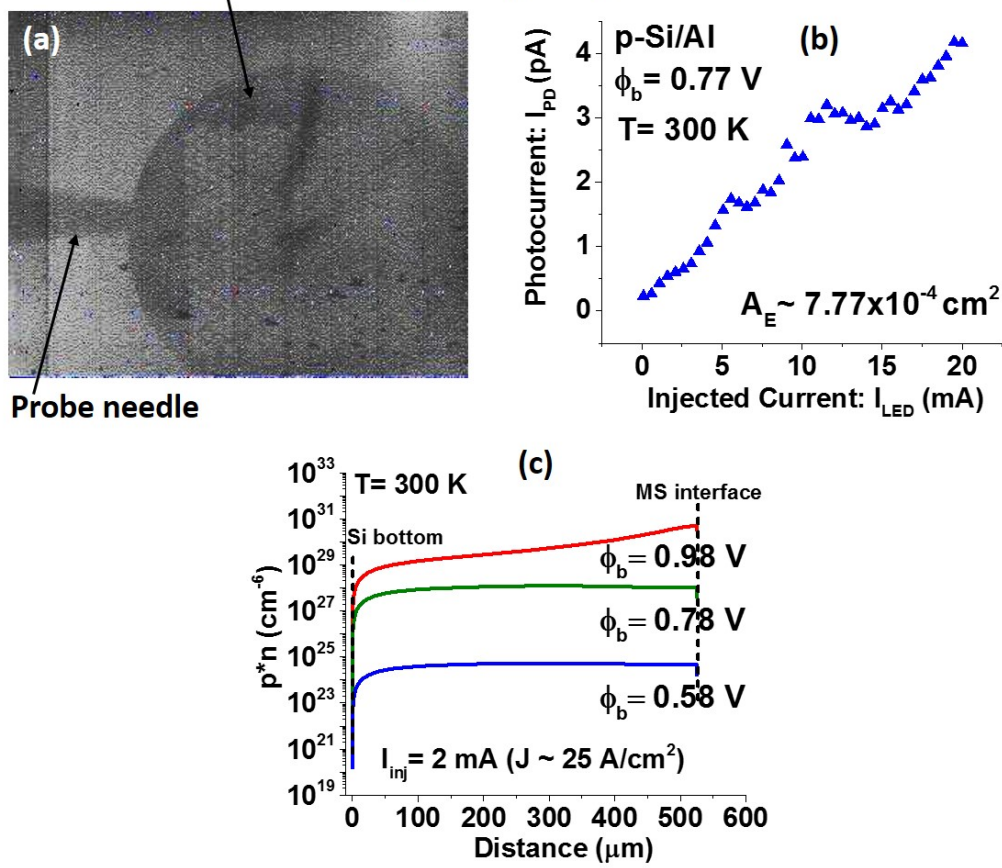

Figure 3.15: (a) EL IR micrograph (in greyscale) of our fabricated p-Si/Al circular diode $\left(\phi_{\mathrm{b}}=0.77 \mathrm{~V}\right)$ with hexagonal openings (see Fig. 3.1(d)). By using an IR camera a faint glow (white regions) was observed from the hexagonal openings, and from the periphery of the circular electrode, (b) Measured short-circuit current $\left(\mathrm{I}_{\mathrm{PD}}\right)$ in the external photodiode $(\mathrm{PD})$ versus injected forward current $\left(\mathrm{I}_{\mathrm{LED}}\right)$ for the same $\mathrm{p}-\mathrm{Si} / \mathrm{Al}$ diode, (c) Simulated $\mathrm{p} \cdot \mathrm{n}$ product at injected current density $\mathrm{J} \sim 25$ $\mathrm{A} / \mathrm{cm}^{2}$ for different $\phi_{\mathrm{b}}$. The $\mathrm{p} \cdot \mathrm{n}$ product increases with $\phi_{\mathrm{b}}$ as expected in high bias or at constant current drive.

However, no measurable light emission ( $\left.\mathrm{I}_{\mathrm{PD}}\right)$ was observed from the lower $\phi_{\mathrm{b}}(0.53 \mathrm{~V}) \mathrm{n}-\mathrm{Si} / \mathrm{Al}$ diode due to the relatively low minority carrier current.

It should be noted that the maximum injected current density, $\mathrm{J}$ $25 \mathrm{~A} / \mathrm{cm}^{2}$ though in high bias range where series resistance or injection limitation becomes important, is still in low injection regime $\left(\mathrm{J} \ll \mathrm{qN} v_{\text {sat }}=\right.$ $1.6 \times 10^{3} \mathrm{~A} / \mathrm{cm}^{2}$, where $\mathrm{N}$ is the active doping concentration and $v_{\text {sat }}$ is the saturation velocity). It is commonly known that at high injection minority carriers do play a role even in a conventional Schottky diode $[1,137,139]$ due to their enhanced drift component as also discussed in section 3.1 before. However, the observation of light emission from the higher- $\phi_{\mathrm{b}}$ Schottky diode at low injection indicates the role of minority carriers in lowering down its series resistance (thus higher injected minority current) as compared to lower- $\phi_{\mathrm{b}}$ diode. This is also in agreement with our simulations where the emitted light intensity which is proportional to the $p \cdot n$ product $[157,158]$ increases for a higher- $\phi_{b}$ at constant injected forward current drive (at high forward biases) as shown in Fig. 3.15(c). The 
Table 3.2: Summary of experimental results.

\begin{tabular}{|c|c|c|c|}
\hline Diode & $\phi_{\mathrm{b}}(\mathrm{V})$ & 2-diode test & EL \\
\hline $\mathrm{p}-\mathrm{Si} / \mathrm{Al}$ & 0.77 & $\Delta \mathrm{I} / \mathrm{I}=37 \%$ & Yes \\
\hline $\mathrm{n}-\mathrm{Si} / \mathrm{Al}$ & 0.53 & $\Delta \mathrm{I} / \mathrm{I}=7 \%$ & No \\
\hline
\end{tabular}

$p \cdot n$ product hence recombination rate is highest near the MS interface.

The results of our experimental investigations from this chapter are summarized in Table 3.2, all indicating the more prominent role of minority carriers in the higher- $\phi_{\mathrm{b}}$ diode as compared to lower- $\phi_{\mathrm{b}}$ diode.

\subsection{Discussion}

Fig. 3.16 summarizes the $\phi_{b}$ dependence of the relative contribution of the diffusion component. As discussed before, for a high- $\phi_{\mathrm{b}}$ Schottky diode, minority carrier diffusion is important and therefore cannot be neglected particularly for reduced substrate thicknesses or a reduced Gummel number in general, and higher temperatures.

The pronounced effect of the minority carrier transport in high- $\phi_{\mathrm{b}}$ Schottky diodes at reduced substrate thicknesses could be attractive for next generation dimensionally-scaled CMOS devices where conventional doping is a challenge. There such Schottky based junctions can be used to realize for instance tunnel devices [96], transistors based on 2-D materials [123] or even low-intensity light emitters for applications such as on-chip communication [159]. In addition, a Schottky based p-n type junction

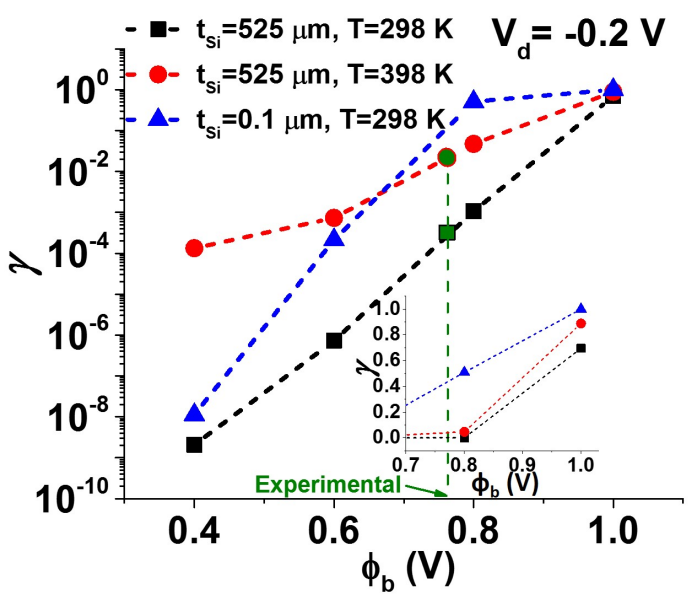

Figure 3.16: (a) Simulated minority carrier injection ratio $\gamma$ as a function of the barrier height $\left(\phi_{\mathrm{b}}\right)$ of the p-Si Schottky diode for different substrate thicknesses and temperatures (Inset: same plot in linear scale at higher $\left.\phi_{b}\right) . \gamma$ has been calculated at $\mathrm{V}_{\mathrm{d}}=-0.2 \mathrm{~V}$ forward bias. The green dashed line indicates our experimentally measured $\phi_{\mathrm{b}}$ for a $525 \mu \mathrm{m}$ thick substrate. 
could be relevant for optoelectronic applications (for example Schottky based UV LEDs [160]) in direct bandgap materials such as GaN, where conventional doping is also not well matured. Moreover, small (sub $100 \mathrm{~nm}$ ) size Schottky contact could further be exploited to enhance the minority carrier effects in undoped bipolar transistor or light emitting devices based on MS junctions.

However from a technological viewpoint, there are several limitations to the pronounced minority carriers effects in practical Schottky diodes. The first one is the availability of required high/low metal work functions to realize high- $\phi_{\mathrm{b}}$ diodes to $\mathrm{n}$-type and $\mathrm{p}$-type semiconductors respectively. This is much of a concern for wider bandgap materials (particularly ntype) as compared to smaller bandgap materials. In this regard, recently popular "carrier selective contacts", such as molybdenum oxide $\left(\mathrm{MoO}_{\mathrm{x}}\right)$ [126], vanadium oxide $\left(\mathrm{V}_{2} \mathrm{O}_{\mathrm{x}}\right)$ or tungsten oxide $\left(\mathrm{WO}_{\mathrm{x}}\right)$ [161, 162] for a "hole selective" and lithium fluoride $\left(\mathrm{LiF}_{\mathrm{x}}\right)$ [163] or $\mathrm{TiO}_{\mathrm{x}}$ [164, 165], for an "electron selective" contact should be explored for realizing extreme$\phi_{\mathrm{b}}$ diodes to n-type and p-type semiconductors respectively, which is otherwise difficult with existing elemental metals. The use of a high work function $\mathrm{MoO}_{\mathrm{x}}$ material to realize high- $\phi_{\mathrm{b}}$ diodes will be discussed in the next chapter.

The second limitation are interface traps the effect of which cannot be ignored for realizing a high- $\phi_{\mathrm{b}}$ Schottky diode. Interface traps influence $\phi_{\mathrm{b}}[1,83]$ and particularly in covalent semiconductors, it is only weakly dependent on $\phi_{\mathrm{m}}$ as the Fermi level is "pinned" at the interface by a large density of surface states. The effective $\phi_{\mathrm{b}}$ will reduce from its ideal value in presence of interface traps, which in turn will decrease the induced inversion charge concentration near the MS interface [46]. Consequently, minority carrier transport would become less important. Interestingly, for ionic semiconductors such as $\mathrm{GaN}, \mathrm{CdS}$ and $\mathrm{ZnS}$, the Fermi-level pinning effect is less pronounced and $\phi_{\mathrm{b}}$ should strongly depend on $\phi_{\mathrm{m}}$ [1].

The use of a thin interfacial tunneling layer has also been proposed earlier to gain better control over a resultant $\phi_{\mathrm{b}}$ and thus induced inversion charge $[46,128-130,151]$. The interfacial layers can further be utilized to suppress the majority current as well and thereby offering a high minority carrier injection efficiency at a given applied bias as reported for metalinsulator-semiconductor (MIS) tunnel diodes [166, 167].

Further, in our simulation study, the effect of Schottky barrier lowering [1] has not been considered which may disturb the injection ratio from the ideal scenario where $\phi_{\mathrm{b}}$ is treated as voltage independent. The barrier lowering is however more pronounced at reverse bias than at forward bias. Moreover, at extreme $\phi_{b}$, the presence of inversion charge results in a sharp drop of the potential near the MS interface and consequently a high surface electric field (see Fig. 3.17). In such scenario, transport mechanisms such as tunneling [1] could be more important than ideally considered TE and diffusion. Previously, Chi et al. [168] reported thermionic-field emission as 

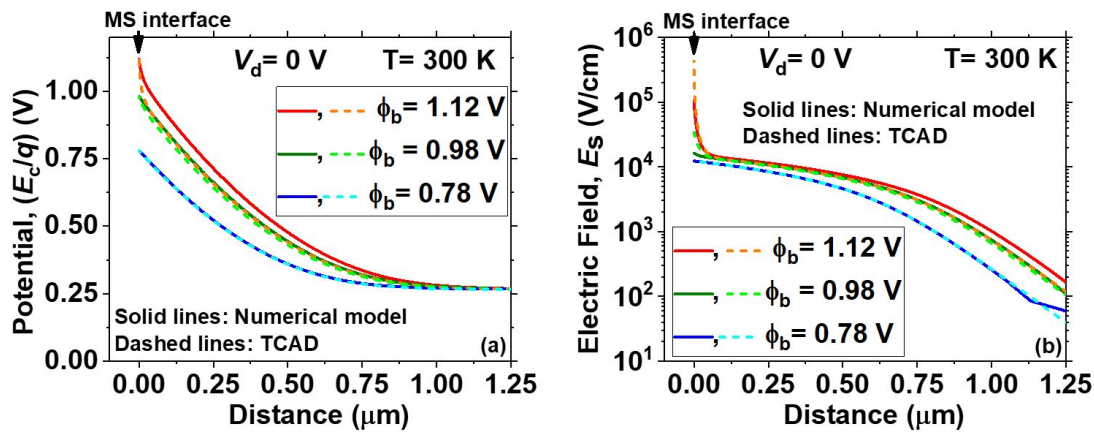

Figure 3.17: TCAD simulated along with numerically calculated, (a) potential and (b) electric field profile in case of n-type Si Schottky diode with varying $\phi_{\mathrm{b}}$ at the MS interface. The numerical model is based on the formulations described in [136] (refer to appendix B) for the solution of Poisson's equation taking the inversion charge into account.

the dominant transport mechanism in NiGe/n-(001) Ge Schottky diodes with $q \phi_{b}$ more than the bandgap. Such formulations should therefore be adapted for more accurate analysis of bipolar charge transport in case of extreme $\phi_{\mathrm{b}}\left(\sim \mathrm{E}_{\mathrm{g}} / \mathrm{q}\right)$ diodes.

\subsection{Conclusion}

In this chapter, the role of the otherwise negligible minority carrier diffusion in high-barrier $\left(\mathrm{q} \phi_{\mathrm{b}}>0.5 \mathrm{E}_{\mathrm{g}}\right)$ Schottky diodes at low injection was discussed using TCAD simulations and experiments with the viewpoint of utilizing such MS contacts for bipolar device applications as an alternative to conventionally doped counterparts. TCAD simulations indicate that in a high- $\phi_{\mathrm{b}}$ Schottky diode with an induced inversion charge, the diffusion current is comparable (or even higher) to the TE current which imparts bipolar characteristics to otherwise unipolar Schottky diode. The diffusion component and therefore injection efficiency can further be improved by reducing the substrate Gummel number (and increasing temperature) making such concepts more suitable for realizing bipolar devices based on UTB semiconductors. In addition, the geometrical effects in Schottky contacts were discussed where the minority carrier injection is enhanced via current spreading. Small-size (sub $100 \mathrm{~nm}$ ) contacts could be exploited for bipolar devices even with nominal $\phi_{\mathrm{b}}$ at lower bias voltages. Moreover, such small-size contacts are more efficient light emitters than their big-size counterparts.

The experimentally observed infrared emission in low injection condition and detected current spreading near the Schottky contact using the 2-diode test methodology all indicate the influence of minority carrier diffusion in a high-barrier Schottky diode, which can be otherwise ignored in a conventional Schottky counterpart. 


\title{
BIPOLAR CHARACTERISTICS OF HIGH-BARRIER PD/ $\mathrm{MOO}_{\chi} / \mathrm{N}-\mathrm{SI}$ DIODES
}

\begin{abstract}
Sub-stoichiometric molybdenum oxide $\left(\mathrm{MoO}_{x}\right)$ has recently been investigated for its application in high efficiency Si solar cells as a "hole selective contact". In this work, the electrical and light-emitting properties of $\mathrm{MoO}_{x}$-based contacts on $\mathrm{Si}$ are investigated from the viewpoint of realizing functional bipolar devices such as LEDs and transistors without any impurity doping of the Si surface. The diodes were realized on n-type Si substrates using e-beam physical vapor deposition of $\mathrm{Pd} / \mathrm{MoO}_{x}$ contacts and their behavior was compared to that of implanted $\mathrm{p}^{+} / \mathrm{n}$-Si diodes. In contrast to a majority-carrier dominated conduction as in conventional Schottky diodes, $\mathrm{Pd} / \mathrm{MoO}_{x} / \mathrm{n}$-Si diodes displayed minority-carrier dominated charge transport with $\mathrm{I}-\mathrm{V}$, $\mathrm{C}-\mathrm{V}$ and light-emitting characteristics comparable to the implanted $\mathrm{p}^{+} / \mathrm{n}$-Si counterparts. Utilizing such $\mathrm{MoO}_{x}$-based contacts, a lateral bipolar transistor concept without employing any doped junctions is also demonstrated. Finally, the limitations of commonly used characterization methods, such as temperature dependent $\mathrm{I}-\mathrm{V}$ and $\mathrm{C}-\mathrm{V}$ measurements, are highlighted for determining the barrier height in case of Schottky diodes with an induced inversion layer at the metalsemiconductor interface.
\end{abstract}

\footnotetext{
This chapter is based on the work submitted in Journal of Applied Physics and is built upon the work presented at the $32^{\text {nd }}$ IEEE International Conference on Microelectronic Test Structures (ICMTS), 2019 [169].
} 


\subsection{Introduction}

The general trend in advanced Si devices is towards lower processing temperatures and more and more shallow junctions. This has shifted the research focus to the development of pn junctions that do not rely on the formation of a heavily-doped surface region on the Si. Ultimately, it would be attractive to form junctions without any chemical doping at all. Schottky diodes fall in this category but conventional Schottky's are unattractive in many situations due to their relatively high saturation currents and poor minority carrier injection. Specifically, for high gain bipolar transistors and in photodiodes where low dark currents are required, other possible solutions should be explored. One solution that has been proposed $[55,56]$ and discussed in the previous chapter is the use of high-barrier Schottky diodes that significantly suppress the majority carrier injection from the substrate and provide a more favorable minority carrier injection ratio in the diode over a wide voltage range. However, experimentally the search for high-barrier Schottky diodes on Si has not been very successful so far.

Other methods of creating $\mathrm{p}^{+} / \mathrm{n}$-like junctions without doping the $\mathrm{n}-\mathrm{Si}$ have been found. Besides high-barrier Schottky diodes, the hole injection into the substrate can also be effectuated by forming a hole inversion layer at an insulator-Si interface that is contacted via doped regions, an example of which are photodiodes that need to be sensitive up to the Si surface [170]. In this case, the holes gather at the surface due to the presence of negative charge in an insulating $\mathrm{Al}_{2} \mathrm{O}_{3}$ layer. Alternatively, an inversion layer at the interface can also be formed by employing a non-insulating layer such as pure boron (PureB). In these "PureB" diodes, the potential responsible for holding the holes at the interface, has been proposed to originate from a high concentration of fixed negative charge created by the B-to-Si bonds [171]. Due to the overall attractive characteristics of this type of junction, it is now widely applied in high performance photodiodes [172].

In this chapter, sub-stoichiometric molybdenum-oxide, $\mathrm{MoO}_{x}$, with $x<3$, based contacts on $\mathrm{n}-\mathrm{Si}$ are studied. $\mathrm{MoO}_{x}$ has been reported before as a high work function $\left(\phi_{\mathrm{m}}\right)$ material $[123,126,173,174]$ that forms a diode to $\mathrm{Si}$ with a low electron injection. Due to these properties, it has recently received attention in Si solar-cells research, being termed as a "hole selective" contact $[126,174]$. More correctly, $\mathrm{MoO}_{x}$ is a wide bandgap transition metal oxide (TMO) [161] with a bandgap $\mathrm{E}_{\mathrm{g}} \sim 3.3 \mathrm{eV}$, and a relatively high electron affinity $>6 \mathrm{eV}$, where an inherent oxygen deficiency results in a defect-band formation near the conduction band [126] (see Fig. 4.1(a)). Thin $\mathrm{MoO}_{x}$ layers were therefore treated as (semi-) metals with a high $\phi_{\mathrm{m}}$. If chemical reactions at the interface with the Si do not play a decisive role [176], such a high $\phi_{\mathrm{m}}$ could be instrumental in the formation of a hole inversion (accumulation) layer on n-type (p-type) Si substrates. Fig. 4.1(b) depicts a schematic energy band diagram of a $\mathrm{MoO}_{x}$ layer on top of n-type $\mathrm{Si}$ with an induced hole inversion layer in $\mathrm{Si}$ at $\mathrm{MoO}_{x} / \mathrm{n}-\mathrm{Si}$ interface. 
(a)

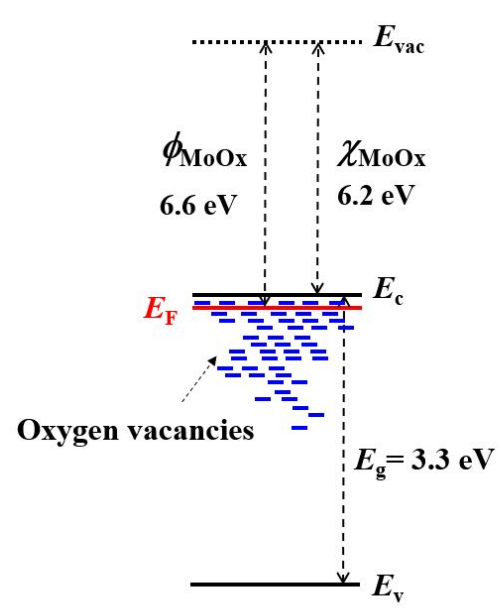

$\mathbf{M o O}_{\mathbf{x}}$

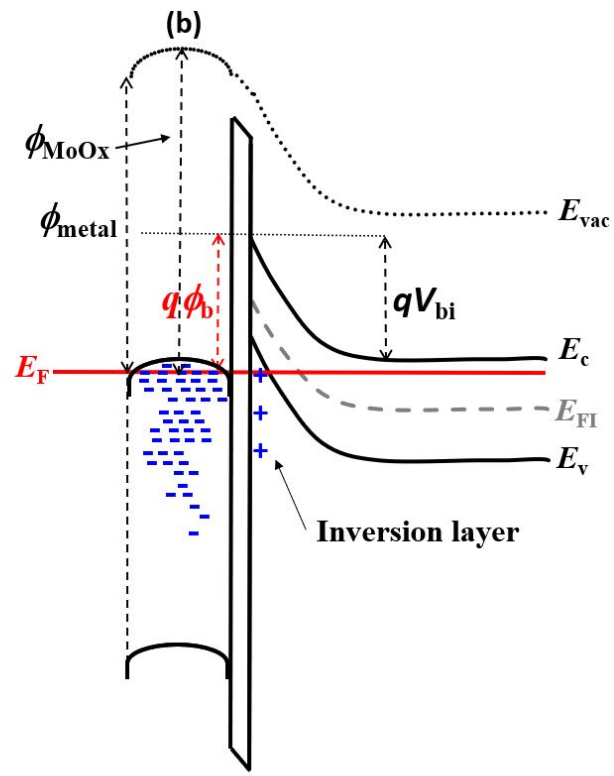

Metal $\mathrm{MoO}_{\mathbf{x}} \mathrm{SiO}_{\mathbf{x}}(\mathbf{M o}) \quad \mathrm{n}-\mathrm{Si}$

Figure 4.1: Schematic energy band diagram at thermal equilibrium of, (a) a vacuum evaporated $\mathrm{MoO}_{x}$ layer with oxygen vacancies [126, 161], (b) metal/ $\mathrm{MoO}_{x} / \mathrm{n}-\mathrm{Si}$ interfaces $[174,175]$. As revealed by TEM analysis, an $\mathrm{SiO}_{x}(\mathrm{Mo})$ interlayer $(\sim 2$ $\mathrm{nm}$ ) is formed as a result of a chemical reaction between $\mathrm{Si}$ and $\mathrm{MoO}_{x}$ during the deposition process.

The presence of a hole inversion layer at the $\mathrm{MoO}_{x} / \mathrm{n}$-Si interface was previously investigated using material characterization approaches such as photoelectron spectroscopy, in conjugation with capacitance-voltage $(\mathrm{C}-\mathrm{V})$ measurements [175, 177]. Also, I- $\mathrm{V}$ measurements along the Si interface of $\mathrm{Pd} / \mathrm{MoO}_{x} / \mathrm{n}$-Si devices were recently performed using dedicated electrical test structures [169] to directly monitor any inversion, but there it was only concluded that if an inversion layer was present it had a sheet resistance higher than $\sim 50 \mathrm{k} \Omega / \square$. However, the diode $\mathrm{I}-\mathrm{V}$ characteristics were found to be similar to that of implanted $\mathrm{p}^{+} / \mathrm{n}$ reference diodes, indicating a strong suppression of the electron injection into the $\mathrm{MoO}_{x}$. In addition, although the $\mathrm{MoO}_{x}$ was conductive, the resistivity was very high with values greater than about $10^{4} \Omega$-cm.

In this chapter the presence of an inversion layer at the $\mathrm{Pd} / \mathrm{MoO}_{x}$ interface to $\mathrm{n}-\mathrm{Si}$ is confirmed. The origin of the hole inversion layer in the $\mathrm{Pd} / \mathrm{MoO}_{x} / \mathrm{n}$-Si and PureB (metal/B/n-Si) diodes may be quite different, but a common property is that the layer on the $\mathrm{Si}$ is conductive albeit with a very high resistivity. Therefore, these diodes will be referred here as metal/non-insulator/semiconductor (MnIS) diodes. The bipolar capabilities of experimental $\mathrm{MoO}_{x}$ based MnIS diodes are examined here, among others, by comparing electrical and optical characteristics to that of implanted $\mathrm{p}^{+} / \mathrm{n}$ reference diodes realized on the same wafer. This includes 
measurement of proof-of-concept surface barrier transistors [54]. In addition, using TCAD simulations and numerical calculations, it is examined whether or not such MnIS diodes can effectively be modeled as high-barrier Schottky diodes.

\subsection{High-barrier Schottky contacts}

The high- $\phi_{\mathrm{b}}$ contact, also referred to as a bipolar-mode Schottky contact [143], can have a significant minority carrier current to total current ratio, $\gamma$, in the whole forward voltage range (refer to chapter 3 ) and therefore may enable applications that cannot be served by conventional Schottky counterparts $[55,56]$. Early reports on the application of such a concept can be traced back to 1953 when Bradley proposed the concept of the surface barrier transistor (SBT) in Ge [54]. The SBT utilizes metal-induced inversion charge to obtain bipolar currents suitable for transistor operation without creating any chemically doped regions.

For an n-type semiconductor, the inversion layer is induced and/or minority carrier effects become important provided that $[46,55]$ :

$$
q \phi_{b n}>E_{g}-k T \ln \frac{N_{v}}{N_{d}}
$$

where $\phi_{b n}$ is the barrier height for electrons, $E_{g}$ is the semiconductor bandgap, $k$ is the Boltzmann constant, $T$ is the temperature, $N_{v}$ is valence band effective density of states, $N_{d}$ is the donor doping density and $q$ is the elementary charge. Eq. (4.1) basically states that the induced inversion charge (hole) density should be higher than the background doping density of the substrate.

Despite the early interest, after the invention of the planar silicon bipolar junction technology, inversion-layer-based device concepts have not attracted much attention. Only recently, due to the strong technological advancement in nanoscale semiconductor devices, alternatives to conventional doping are being sought. In this context, high- $\phi_{\mathrm{b}}$ MS contacts could be relevant for electrostatic doping (ED) $[46,48]$. In ultra-thin body (UTB) devices such 2-D surface inversion could effectively result in volume inversion [178]. Both in bulk and UTB semiconductors, such Schottky contacts could be interesting for realizing dopant-free electrical and optical devices. In previous chapter, minority carrier effects were discussed in an $\mathrm{Al} / \mathrm{p}-\mathrm{Si}$ diode where $\phi_{\mathrm{b}}, \sim 0.78 \mathrm{~V}$, was moderately high [56]. It is therefore worthwhile to investigate in this chapter if an extreme work function property of $\mathrm{MoO}_{x}$ can be exploited to realize high-barrier Si diode with bipolar conduction properties. 


\subsection{Experimental procedure}

The basic device structure is shown in Fig. 4.2 for the ring-shaped test structures used for both diode and sheet resistance measurements [169] ${ }^{1}$. For $\mathrm{C}-\mathrm{V}$ measurements, large square-shaped structures with an area of $A_{E}=3.1 \times 10^{-3} \mathrm{~cm}^{2}$ were used. Large structures suitable for measuring lateral bipolar transistors and light-emission were also included. The substrates were n-type (100) Si wafers with a resistivity of 1-10 ohm-cm covered with a $235 \mathrm{~nm}$ thick thermal oxide. Implantations of $\mathrm{B}^{+}$annealed in argon at $950^{\circ} \mathrm{C}$, were used to form $\mathrm{p}^{+}$regions with a junction depth of $0.5 \mu \mathrm{m}$ and a surface doping of $10^{19} \mathrm{~cm}^{-3}$. Windows were then wet-etched in the oxide to give access to both implanted and non-implanted regions. A resist coating suitable for lift-off was used to pattern a layer stack of $\mathrm{Pd} / \mathrm{MoO}_{x}$ deposited in an e-beam physical vapor deposition (EBPVD) system. For the $\mathrm{MoO}_{x}$, a $99.95 \%$ pure material from Kurt J. Lesker in 3-6 mm large pieces was placed in a Fabmate crucible of $99.95 \%$ elemental carbon. $\mathrm{MoO}_{x}$ layer with a thickness of $\sim 7-8 \mathrm{~nm}$ was grown at an evaporation rate of about $0.05 \mathrm{~nm} / \mathrm{s}$. Before deposition, the evaporation chamber was pumped down to the base pressure of about $1.5 \times 10^{-7}$ mbar. The $\mathrm{MoO}_{x}$ layer was then capped with a Pd layer of about $120 \mathrm{~nm}$ thick in the same deposition chamber without breaking the vacuum. A lift-off process was used to remove the $\mathrm{Pd} / \mathrm{MoO}_{x}$ layer stack from the $\mathrm{Si}$ at places other than oxide windows. As a last step, the backside of the substrate was coated with Al.

The Pd has a relatively low resistivity and this prevents the use of the structures described above to measure the sheet resistance of the inversion layer along the surface of the $\mathrm{Si}$. Therefore, another sample was prepared where the Pd was replaced by a $10 \mathrm{~nm}$ thick B (boron) layer. The B is known to be chemically very inert in many situations [172] and is expected to protect the $\mathrm{MoO}_{x}$ from oxidation in air. Moreover, the resistivity of $\mathrm{B}$ is so high, $>500 \Omega-\mathrm{cm}[179]$, that it is not likely to influence the sheet resistance measurement. For the B deposition, a $99.5 \%$ pure material from Kurt J. Lesker in the form of cylindrical ingots was utilized. No thermal annealing was performed on any of the prepared samples. The structure of the fabricated layer stacks was examined using transmission electron microscopy (TEM, Philips CM300ST-FEG).

All measurements were carried out in the dark and on a temperaturecontrolled chuck. The electrical measurements were performed using a Keithley 4200 semiconductor characterization system with a preamplifier. For the optical spectrum measurements, an Avaspec UV-Vis/NIR spectrometer from Avantes was used. IR micrographs were captured using a cooled InGaAs detector based camera (XEVA-320 from Xenics).

\footnotetext{
${ }^{1}$ The device fabrication part reported in this chapter was performed by D.T. Shivakumar.
} 

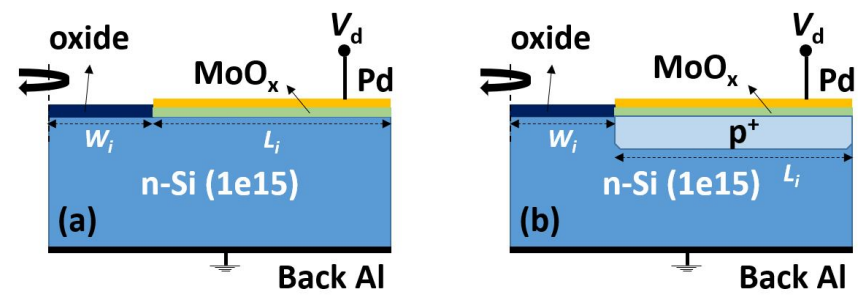

Figure 4.2: (a) Schematic cross-sections of the fabricated ring-shaped devices, (a) $\mathrm{Pd} / \mathrm{MoO}_{x} / \mathrm{n}$-Si diode, and (b) an implanted $\mathrm{p}^{+} / \mathrm{n}$-Si diode both with a the ring length $\mathrm{L}_{\mathrm{i}}=200 \mu \mathrm{m}$, width $W_{\mathrm{i}}=64 \mu \mathrm{m}$ and area $A_{E}=2 \times 10^{-3} \mathrm{~cm}^{2}$. The vertical axis of symmetry is at the left hand side indicated by a dashed line.

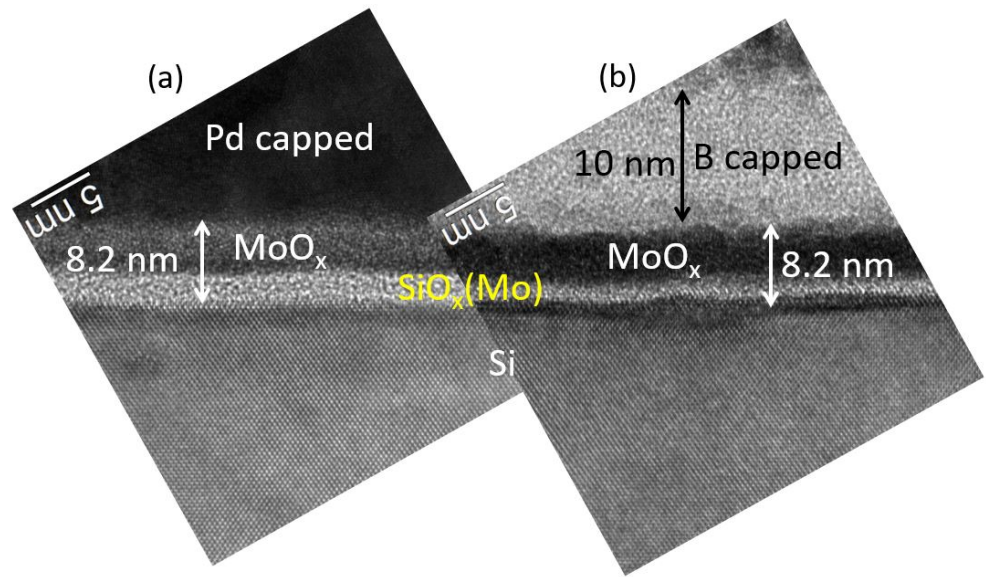

Figure 4.3: TEM cross-sectional images of the fabricated samples, (a) $\mathrm{Pd} / \mathrm{MoO}_{x} / \mathrm{n}-$ $\mathrm{Si}$ and (b) $\mathrm{B} / \mathrm{MoO}_{x} / \mathrm{n}-\mathrm{Si}$.

\subsection{Material analysis}

TEM images of the $\mathrm{Pd} / \mathrm{MoO}_{x} / \mathrm{n}-\mathrm{Si}$ and $\mathrm{B} / \mathrm{MoO}_{x} / \mathrm{n}$-Si layer stacks are shown in Fig. 4.3. At the interface with the $\mathrm{Si}$, an amorphous interlayer of about $2 \mathrm{~nm}$ in thickness is observed in both cases. Such a layer was also reported in other studies [175] where energy-filtered TEM (EF-TEM) analysis revealed a composition of $\mathrm{Si}, \mathrm{O}$ and Mo atoms, referred to as a hybrid a-SiO${ }_{x}(\mathrm{Mo})$ layer. The accompanying $\mathrm{SiO}_{x}(\mathrm{Mo})$ interlayer, which is possibly formed during the deposition of $\mathrm{MoO}_{x}$ on $\mathrm{Si}$, could have multiple roles in the charge carrier transport in our devices such as passivating the $\mathrm{Si}$ surface, hence suppressing the electron current and inducing a hole inversion layer. Further, the sub-stiochiometric nature of the e-beam deposited $\mathrm{MoO}_{x}$ thin films was confirmed by X-ray photoelectron spectroscopy (XPS) analysis (not shown) which indicates $x=2.42 \pm 0.24$ that is in close agreement with other reports $[162,175]$. The bipolar-mode diode characteristics of the $\mathrm{MoO}_{x}$ based MnIS diodes as studied by electrical and light emission measurements will be treated in the following sections. 


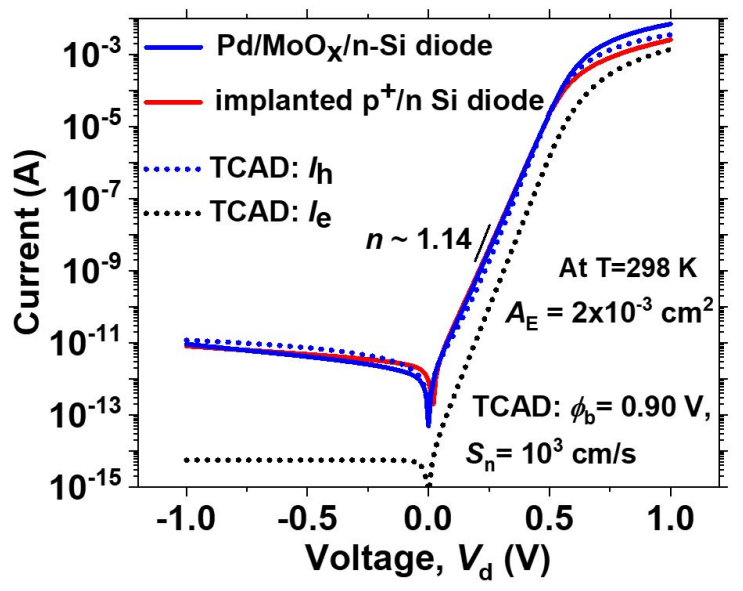

Figure 4.4: $\mathrm{I}-\mathrm{V}$ characteristics of a fabricated $\mathrm{Pd} / \mathrm{MoO}_{x} / \mathrm{n}-\mathrm{Si}$ diode and implanted $\mathrm{p}^{+} / \mathrm{n}$ Si reference diode at room temperature. For comparison TCAD simulation data of Si Schottky diode with an identical geometry modeled using $\phi_{\mathrm{b}}=0.90 \mathrm{~V}$ and $S_{n}=10^{3} \mathrm{~cm} / \mathrm{s}$ is shown. Other fitting parameters used for simulations: SRH lifetimes, $\tau_{\mathrm{n}}=10 \mu \mathrm{s}, \tau_{\mathrm{p}}=20 \mu \mathrm{s}$ and $\mathrm{N}_{\mathrm{D}}=5 \times 10^{14} \mathrm{~cm}^{-3}$.

\subsection{Diode I $-V(-T)$ characteristics}

Typical I - V characteristics of the fabricated $\mathrm{Pd} / \mathrm{MoO}_{x} / \mathrm{n}$-Si MnIS diodes are shown in Fig. 4.4. The diode displays a high rectification of $\sim 10^{8}$ at $V_{d}= \pm 1 \mathrm{~V}$, a very low leakage current density of about $5 \mathrm{nA} / \mathrm{cm}^{2}$ and an ideality factor $n \sim 1.14$. The $n$ value close to unity indicates that charge transport in the diode is mainly governed by processes such as drift-diffusion or thermionic emission. The small non-ideality could be attributed to process related defects or contamination.

Also shown in Fig. 4.4 are the I - V characteristics of an implanted $\mathrm{p}^{+} / \mathrm{n}$ reference diode. The current levels of the two diodes are almost identical. In the latter, the current is diffusion limited and the hole injection is dominant because the Gummel number of the $\mathrm{p}^{+}$region is about 10 to 100 times higher than the substrate Gummel number. Moreover, the hole diffusion current of the two diodes should be the same as both are determined by the same substrate Gummel number. This implies that the hole current is dominant even in the $\mathrm{Pd} / \mathrm{MoO}_{x} / \mathrm{n}$-Si diode and the electron current is of the same order or lower. The observed high minority carrier injection ratio $(\gamma)$ in the $\mathrm{Pd} / \mathrm{MoO}_{x} / \mathrm{Si}$ Si diodes indicates the role of the high-barrier and associated hole inversion layer at the $\mathrm{MoO}_{x} / \mathrm{Si}$ interface. In addition, the passivating nature of $\mathrm{MoO}_{x}$ based contact on $\mathrm{Si}[175,180]$, possibly originating from the observed $\mathrm{SiO}_{x}(\mathrm{Mo})$ interlayer, with a low surface recombination velocity, could also play a role in suppressing the TE further as confirmed by TCAD simulations.

TCAD simulation of the $\mathrm{Pd} / \mathrm{MoO}_{\mathrm{x}} / \mathrm{n}$-Si diode as a Si Schottky diode shows good agreement with the experimental results as indicated in Fig. 

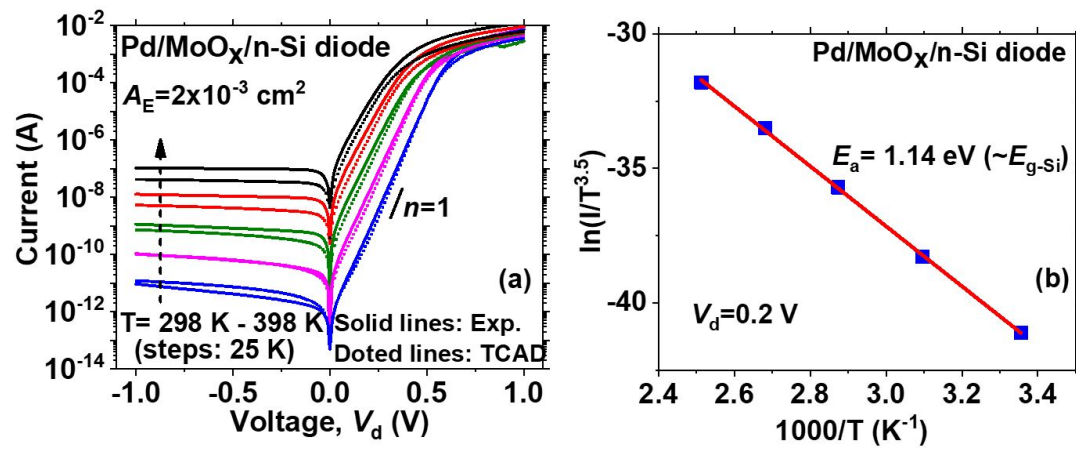

Figure 4.5: (a) Temperature dependent I $-\mathrm{V}$ characteristics of the fabricated $\mathrm{Pd} / \mathrm{MoO}_{x} / \mathrm{n}$-Si diode and their comparison with those of the simulated Si Schottky diode. (b) Arrhenius plot of the experimental device at $V_{d}=0.2 \mathrm{~V}$ forward bias to estimate the activation energy $E_{a}$.

4.4 and Fig. 4.5(a). For this purpose, the Schottky electrode and its interface to $\mathrm{Si}$ was modeled using the lower limit of the barrier height, $\phi_{\mathrm{b}}=0.90 \mathrm{~V}$ as extracted from the $\mathrm{C}-\mathrm{V}$ analysis (section 4.7 ), and an effective surface recombination velocity for electrons, $S_{n}=10^{3} \mathrm{~cm} / \mathrm{s}$. Such relatively low value of $S_{n}$ is typical for passivated $\mathrm{SiO}_{x} / \mathrm{Si}$ interfaces [181] and is incorporated in our simulations to mimic more complex $\mathrm{MoO}_{x} / \mathrm{Si}$ junctions [182]. For a given value of $\phi_{b}$ and $S_{n}, I_{h}$ determines the I $-V$ characteristics as also observed experimentally, while $I_{e}$ appears to be one order of magnitude lower than $I_{h}$. The $I_{h}$ is mainly determined by the substrate Gummel number while $\phi_{\mathrm{b}}$ and $S_{\mathrm{n}}$ affect only $\mathrm{I}_{\mathrm{e}}$. It is possible to model the hole dominated experimental I $-V$ characteristics by other suitable combinations of $\phi_{\mathrm{b}}$ $(>0.90 \mathrm{~V})$ and $S_{n}$ such that $I_{e}$ falls sufficiently below $I_{h}$. Nevertheless, the simulations confirmed the important role of a sufficiently high $\phi_{\mathrm{b}}$ and well passivated $\mathrm{MoO}_{x} / \mathrm{Si}$ interface in the observed I $-\mathrm{V}$ characteristics of $\mathrm{Pd} / \mathrm{MoO}_{x} / \mathrm{n}-\mathrm{Si}$ diodes.

As shown in Fig. 4.5(a), temperature dependent I - V measurements were also performed in order to analyze the dominant charge transport mechanism and to extract the activation energy, $E_{a}$. In the case of thermionicemission dominated transport $\left(\mathrm{I} \propto \exp \left(-\mathrm{q} \phi_{\mathrm{b}} / \mathrm{kT}\right)\right)$, as in conventional Schottky diodes, the $E_{a}$ obtained from Arrhenius plots would give the effective $\phi_{\mathrm{b}}$ at the MS junction. As opposed to this, in case of diffusiondominated transport $\left(I \propto \exp \left(-E_{g} / k T\right)\right)$, an $E_{a}$ corresponding to the semiconductor bandgap, $\mathrm{E}_{\mathrm{g}}$, is expected. The Arrhenius plot in Fig. 4.5(b) for the $\mathrm{Pd} / \mathrm{MoO}_{x} / \mathrm{n}$-Si diode at forward bias $\left(V_{d}=0.2 \mathrm{~V}\right)$ displays an $E_{a}$ corresponding to the $\mathrm{Si} \mathrm{E}_{\mathrm{g}}$. Similar results were obtained for the implanted $\mathrm{p}^{+} / \mathrm{n}$ Si diode. This confirms that hole injection is indeed dominant in our $\mathrm{Pd} / \mathrm{MoO}_{x} / \mathrm{n}$-Si diodes at forward bias. These results also underline that it is not possible to determine the actual value of $\phi_{b}$ using I $-V-T$ measurements in such high-barrier diodes where diffusion dominates the current rather than TE. 


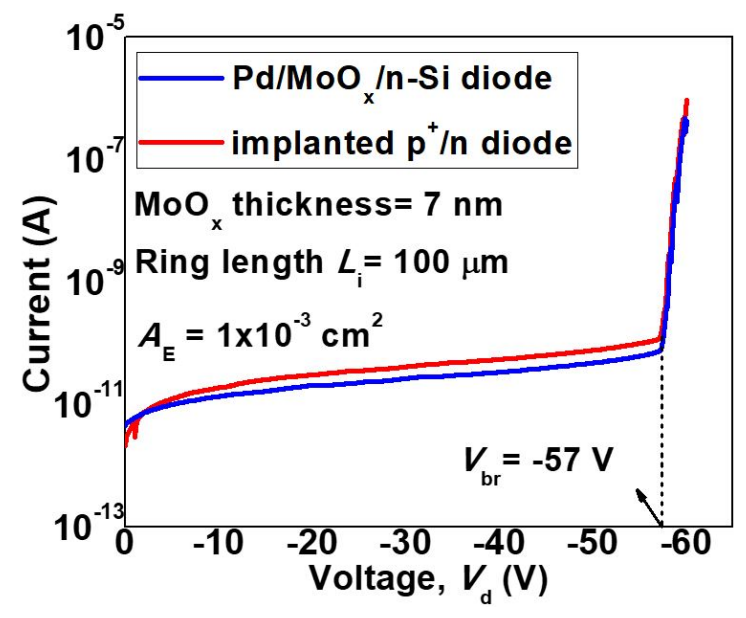

Figure 4.6: Reverse bias I - V characteristics of a fabricated $\mathrm{Pd} / \mathrm{MoO}_{x} / \mathrm{n}$-Si diode at room temperature compared to the implanted $\mathrm{p}^{+} / \mathrm{n}-\mathrm{Si}$ reference diode.

Furthermore, as shown in Fig. 4.6, the reverse I-V characteristics of the $\mathrm{Pd} / \mathrm{MoO}_{x} / \mathrm{n}$-Si diode were also found to be similar to that of implanted $\mathrm{p}^{+} / \mathrm{n}$ Si diodes with identical breakdown voltages, $\mathrm{V}_{\mathrm{br}}=-57 \mathrm{~V}$. This strongly indicates the presence of a hole inversion layer in the $\mathrm{Pd} / \mathrm{MoO}_{x} / \mathrm{n}$-Si diode that prevents the expansion of the depletion layer into the $\mathrm{MoO}_{x}$ layer. Moreover, the breakdown characteristics are independent of the $\mathrm{MoO}_{x}$ layer thickness when increased from $5 \mathrm{~nm}$ to $33 \mathrm{~nm}$, which further suggests that the depletion width is solely determined by the substrate doping. By calculating the depletion width at breakdown, the charge displaced in the substrate was estimated to be about $8.6 \times 10^{11} \mathrm{~cm}^{-2}$ which is equivalent to the corresponding charge in the p-type region. This value represents a lower limit of the hole concentration in the inversion layer at the $\mathrm{MoO}_{x} / \mathrm{n}-$ Si interface.

\subsection{Sheet resistance measurements}

By using the $\mathrm{MoO}_{x}$ samples prepared with the high-resistivity B capping layer, it was possible to measure the sheet resistance along Si surface under the deposited $\mathrm{MoO}_{x}$. Following the same methodology as outlined before [169], $R_{\text {sh }}$ values in the range of $60-100 \mathrm{k} \Omega / \square$ were extracted (refer to appendix D). This is much lower than the sheet resistance of the thin $B$ layer that is at least $1 \mathrm{M} \Omega / \square$ and therefore does not contribute in any significant way. Using simulations to translate the $R_{\mathrm{sh}}$ into a value for the hole concentration in the inversion layer is not directly possible because the effective mobility at the $\mathrm{MoO}_{x} / \mathrm{Si}$ interface is difficult to predict. Nevertheless, the measured $R_{\mathrm{sh}}$ does not conflict with the lower limit of $8.6 \times 10^{11} \mathrm{~cm}^{-2}$ found in the previous section. 

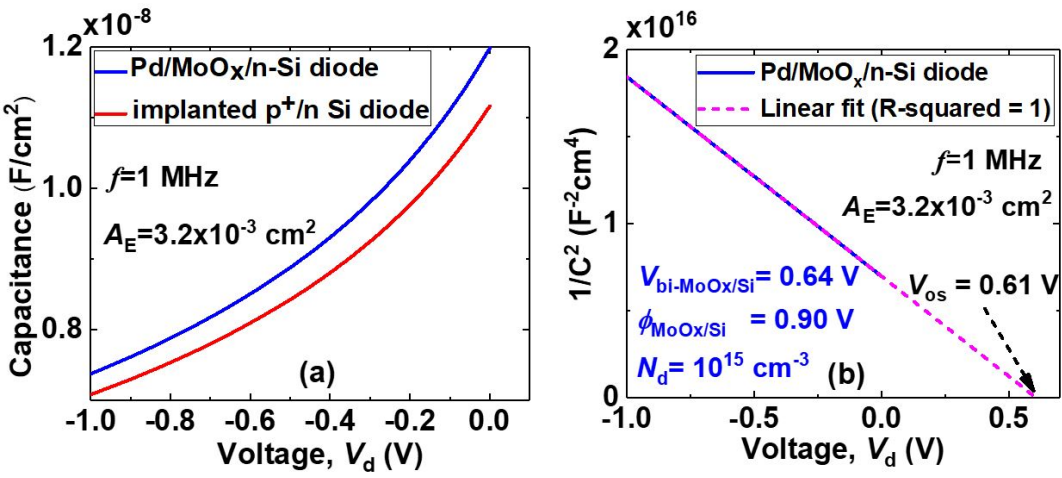

Figure 4.7: (a) Reverse bias $\mathrm{C}-\mathrm{V}$ characteristics of the fabricated $\mathrm{Pd} / \mathrm{MoO}_{x} / \mathrm{n}-\mathrm{Si}$ and implanted $\mathrm{p}^{+} / \mathrm{n}$-Si diodes at room temperature, (b) $(1 / \mathrm{C})^{2}$ as a function of applied voltage indicating the estimated built-in potential, $\mathrm{V}_{\mathrm{bi}}$ for the $\mathrm{Pd} / \mathrm{MoO}_{x} / \mathrm{n}$ Si diode extracted by linear-fitting.

\section{7 $\quad \mathrm{C}-\mathrm{V}$ measurements}

In addition to temperature dependent $\mathrm{I}-\mathrm{V}$ measurements, $\mathrm{C}-\mathrm{V}$ characteristics measured with reverse biasing are commonly used to extract the $\phi_{\mathrm{b}}$ of Schottky diodes. The measured $\mathrm{C}-\mathrm{V}$ profile for a $\mathrm{Pd} / \mathrm{MoO}_{x} / \mathrm{n}-\mathrm{Si}$ diode are shown in Fig. 4.7(a) along with those of the implanted $\mathrm{p}^{+} / \mathrm{n}$-Si diode. Notably, the two diodes show similar expansion of the depletion region with increasing reverse voltage, with only a small difference that could be attributed to differences in the $\mathrm{p}$ to $\mathrm{n}$ transition profile of the implanted and $\mathrm{MoO}_{x} / \mathrm{n}-\mathrm{Si}$ junctions.

Following a conventional approach [1], the built-in potential $\left(\mathrm{V}_{\mathrm{bi}}\right)$ and in turn $\phi_{\mathrm{b}}$ of the diode can be estimated from the offset voltage, $V_{\mathrm{os}}$, of the $\mathrm{C}^{-2}-\mathrm{V}$ plot. From the measurements shown in Fig. 4.7(b), $\mathrm{V}_{\mathrm{bi}}$ of about $0.64 \mathrm{~V}$ was extracted for our $\mathrm{Pd} / \mathrm{MoO}_{x} / \mathrm{n}$-Si diode with a resulting barrier height $\left(\phi_{\mathrm{MoOx} / \mathrm{Si}}\right)$ of about $0.90 \mathrm{~V}$ at the $\mathrm{MoO}_{x} / \mathrm{n}-\mathrm{Si}$ interface. However, this formulation is only valid when there is only depletion charge near an MS interface. Here, the extracted $\phi_{b}$ indicates that the interface may be inverted as predicted by Eq. (4.1) which sets the strong inversion condition at $\phi_{\mathrm{b}}>0.85 \mathrm{~V}$ when $\mathrm{N}_{\mathrm{d}}=10^{15} \mathrm{~cm}^{-3}$.

When an inversion layer is present, another formulation should be adopted based on the solution of Poisson's equation with the inclusion of the inversion charge [136, 183]. Schwarz and Walsh [136] have previously shown that for a sufficiently high barrier, the measured capacitance is no longer sensitive to the barrier height and is largely determined by the resistivity of the substrate. The inversion charge basically "screens" the depletion charge. Gummel and Scharfetter [184] also reached a similar conclusion for an abrupt (single-sided) $\mathrm{p}^{+} / \mathrm{n}$ junction where the $\mathrm{V}_{\mathrm{os}}$ was found to be nearly independent of $N_{a}$ for large $N_{a} / N_{d}$ ratios. Therefore, in the presence of inversion charge in a Schottky junction or even in case of a 


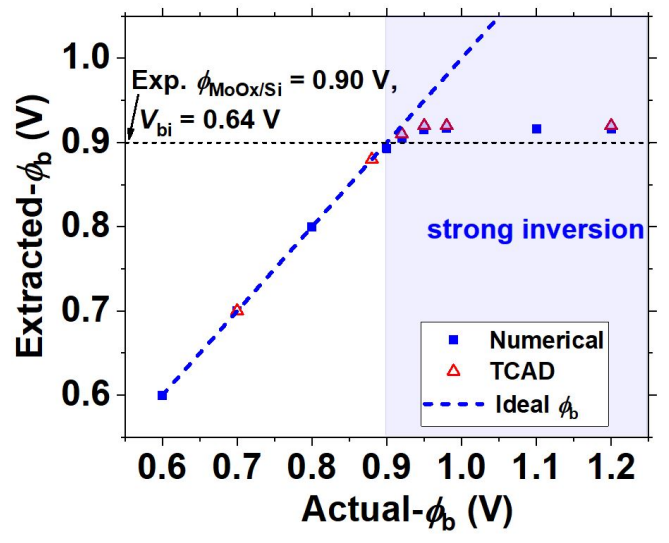

Figure 4.8: The extracted $\phi_{\mathrm{b}}$ as a function of the actual $\phi_{\mathrm{b}}$, obtained from a numerical solution of Poisson's equation in the presence of a hole inversion layer and compared with TCAD simulations. The black dashed line indicates the position of our experimentally extracted $\phi_{\mathrm{MoOx} / \mathrm{Si}}$ value.

single sided $\mathrm{p}^{+} / \mathrm{n}$ junction, the $\mathrm{V}_{\mathrm{os}}$ from the $C^{-2}-V$ plot can no longer be used to accurately determine the $V_{\mathrm{bi}}$ or $\phi_{\mathrm{b}}$ of the junction.

In order to investigate the influence of inversion charge on the extracted $V_{\mathrm{bi}}$ for our device, the methodology as outlined in [136] was adapted and the solution of Poisson's equation in presence of a hole inversion layer was re-visited (refer to appendix $B$ ), for a more generalized case where $N_{c} \neq N_{v}$. From a numerical solution, $\mathrm{C}^{-2}-\mathrm{V}$ plots were first calculated for various $\phi_{\mathrm{b}}$ in the reverse bias range of $0-1 \mathrm{~V}$. The $\mathrm{V}_{\mathrm{os}}$ extracted from linear fitting was then used to determine $\phi_{\mathrm{b}}$.

Fig. 4.8 shows the extracted $\phi_{\mathrm{b}}$ using this numerical approach as a function of the actual $\phi_{\mathrm{b}}$. The extracted $\phi_{\mathrm{b}}$ tends to saturate beyond a certain value $(\sim 0.90 \mathrm{~V})$ where the influence of inversion charge starts to become important. The numerical solution is also in good agreement with TCAD simulations [98]. In a more strict sense, which is practically masked by the experimental and computational inaccuracies, the $C^{-2}-V$ plot in the presence of inversion charge becomes non-linear and $V_{o s}$ is then a function of the applied voltage [136, 184]. Therefore, it can be concluded that for a sufficiently high barrier, the actual $\phi_{\mathrm{b}}$ cannot be obtained using the $\mathrm{C}-\mathrm{V}$ analysis. In such a scenario, $\mathrm{V}_{\mathrm{os}}$ at reverse voltages close to zero, could only give an estimate of the lower limit of $\phi_{\mathrm{b}}$ [184].

Hence, our experimentally calculated $\phi_{\mathrm{MoOx} / \mathrm{Si}}$ value of $0.90 \mathrm{~V}$ may not correspond to the actual $\phi_{\mathrm{b}}$ but is an indicator of the lower limit. For $\phi_{\mathrm{b}}=0.90 \mathrm{~V}$, a peak hole concentration at the interface was estimated to be about $5 \times 10^{15} \mathrm{~cm}^{-3}$ as given by $\left.p_{0}=N_{\mathrm{v}} \exp \left(-\left(\mathrm{E}_{\mathrm{g}}-\phi_{\mathrm{b}}\right) / \mathrm{kT}\right)\right)$ which leads to an effective sheet carrier density, $p_{\mathrm{sh}} \sim 10^{10} \mathrm{~cm}^{-2}$. The obtained value of $p_{\text {sh }}$ here is, however, about two orders of magnitude lower than the one estimated from reverse breakdown characteristics which further indicates that the actual $\phi_{\mathrm{b}}$ at the $\mathrm{MoO}_{x} / \mathrm{n}-\mathrm{Si}$ interface is indeed higher than $0.90 \mathrm{~V}$. 

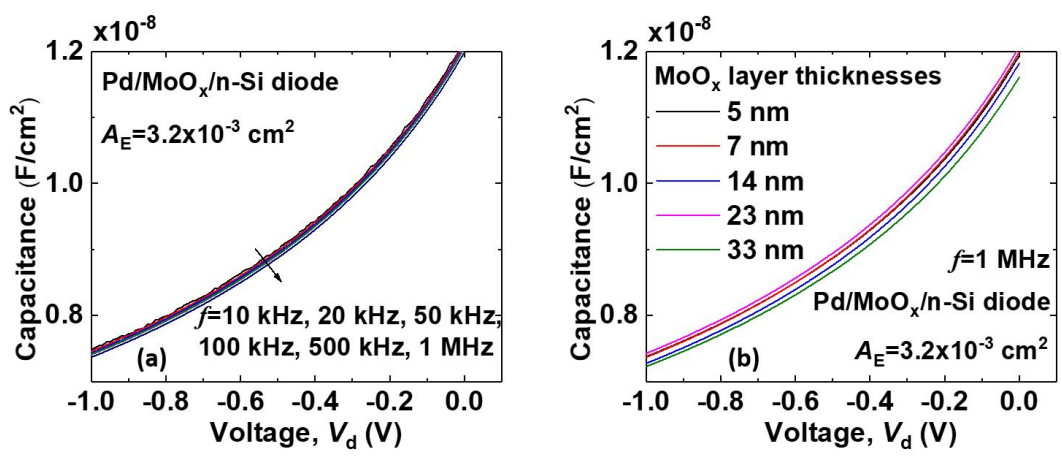

Figure 4.9: Reverse bias $\mathrm{C}-\mathrm{V}$ characteristics of a fabricated $\mathrm{Pd} / \mathrm{MoO}_{x} / \mathrm{n}-\mathrm{Si}$ diode, (a) at various frequencies, and (b) for various $\mathrm{MoO}_{x}$ layer thicknesses at fixed frequency of $1 \mathrm{MHz}$.

In addition, $\mathrm{C}-\mathrm{V}$ measurements at multiple frequencies were performed as shown in the Fig. 4.9(a). The capacitance remains practically constant for varying frequencies which rules out the possibility of any trapped charge in the $\mathrm{MoO}_{x}$ layer influencing the $\mathrm{C}-\mathrm{V}$ measurements. Furthermore, the $\mathrm{C}-\mathrm{V}$ measurements in Fig. 4.9(b) of our samples, show no systematic change in the capacitance for various $\mathrm{MoO}_{x}$ thicknesses. This suggests that the depletion is occurring mainly in the $\mathrm{Si}$, and that the $\mathrm{MoO}_{x}$ layer is behaving more like a metal or degenerate semiconductor with some finite series resistance as was reported earlier [123, 126]. A similar conclusion was drawn before [169], where the diode I - V characteristics hardly changed with the $\mathrm{MoO}_{x}$ layer thickness except at high forward voltages where the series resistance through the $\mathrm{MoO}_{x}$ layer attenuated the current. These experimental observations indicate that only the $\mathrm{MoO}_{x} / \mathrm{Si}$ interface is determining the device characteristics and the bulk $\mathrm{MoO}_{x}$ layer is providing the contact to the metal capping layer.

\subsection{Light-emission measurements}

Forward-bias light emission in Schottky diodes occurs as a result of radiative recombination of injected minority carriers with background majority carriers. The minority current therefore determines the radiative recombination rate in the device. In conventional Schottky diodes, the minority current gets suppressed before it reaches values high enough for any measurable light output due to its poor supply from the contact or series resistance. Therefore Schottky diodes are generally disregarded for light emitting applications. However, a high-barrier diode with an induced inversion layer can sustain a high minority current that, as observed in the previous sections, can become comparable to that of doped junction counterparts.

Light-emission measurements on our fabricated diodes were performed. The $\mathrm{Pd} / \mathrm{MoO}_{x} / \mathrm{n}-\mathrm{Si}$ diodes show clear electroluminescence (EL) during 

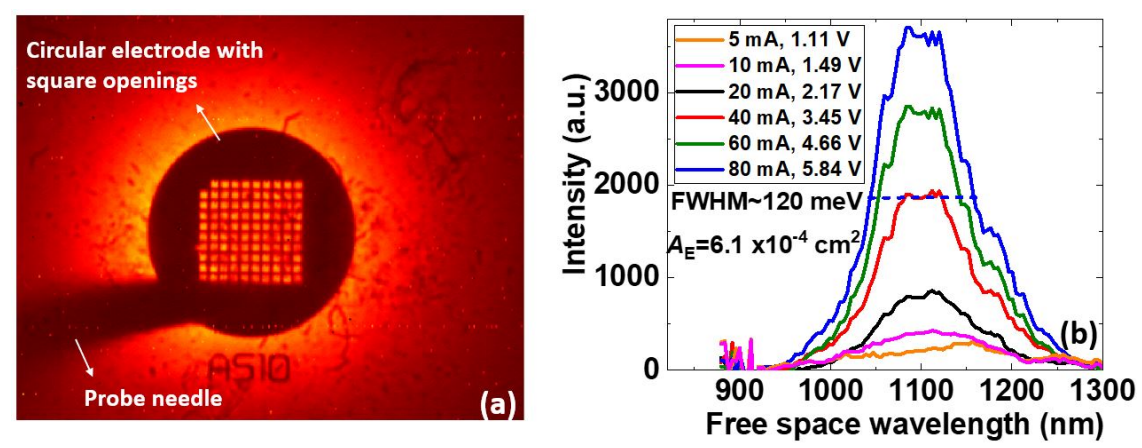

Figure 4.10: (a) Bright field EL IR micrograph of the fabricated $\mathrm{Pd} / \mathrm{MoO}_{x} / \mathrm{n}-\mathrm{Si}$ diode at a constant forward current drive of $100 \mathrm{~mA}\left(\mathrm{~J}=165 \mathrm{~A} / \mathrm{cm}^{2}\right)$, (b) Optical spectrum of the emitted light from the same diode at various forward current levels.

forward bias operation as is evident from the IR micrograph in Fig. 4.10(a). The IR emission is centered around the wavelength corresponding to the $\mathrm{Si}$ bandgap, i.e. $1.12 \mu \mathrm{m}$ as shown in Fig. 4.10(b). As expected, the emitted EL intensity increases with the injected current level. The observed full-widthhalf-maximum (FWHM) in excess of $\sim 1.8 \mathrm{kT}$ (Ref. [157]) is attributed to the indirect bandgap in $\mathrm{Si}$ where involvement of phonons in the radiative recombination process leads to the EL-broadening.

Moreover, the maximum injected current density $\left(\mathrm{J}=165 \mathrm{~A} / \mathrm{cm}^{2}\right)$ here falls in the low injection regime $\left(\mathrm{J} \ll \mathrm{qN} v_{\text {sat }}=1.6 \times 10^{3} \mathrm{~A} / \mathrm{cm}^{2}\right.$ where $\mathrm{N}$ is the active doping concentration and $v_{\text {sat }}$ is the saturation velocity). Compared to the $\mathrm{p}^{+} / \mathrm{n}$ reference diodes, the light emission in the $\mathrm{Pd} / \mathrm{MoO}_{x} / \mathrm{n}-\mathrm{Si}$ diode is about the same, being about 1.5 times less bright. Some discrepancy in the observed brightness between the two diodes could be related to a possible difference in their extraction efficiencies caused by their different junction depths, since the $p \cdot n$ product and thus the light emission is highest at the junction. The junction depth in case of the implanted $\mathrm{p}^{+} / \mathrm{n}$ diode is about $0.5 \mu \mathrm{m}$ below the surface and would therefore experience relatively low optical losses at the top electrode compared to the $\mathrm{Pd} / \mathrm{MoO}_{x} / \mathrm{n}-\mathrm{Si}$ diode, where light is emitted very close to the electrode. Nevertheless, such a high-barrier Si diode which shows EL comparable to that of the implanted $\mathrm{p}^{+} / \mathrm{n}$-Si diode could potentially be utilized to realize low-intensity Si based light emitters for applications such as on-chip communication [159].

\subsection{Surface barrier transistor (SBT) measurements}

A lateral bipolar transistor, referred to as a surface barrier transistor (SBT) [54], was realized using the high-barrier $\mathrm{MoO}_{x}$ contacts. Fig. 4.11(a) shows a schematic cross section of the experimental device where two closely spaced top contact electrodes were utilized as emitter (E) and collector (C), while the metalized substrate back surface served as the base contact (B). 

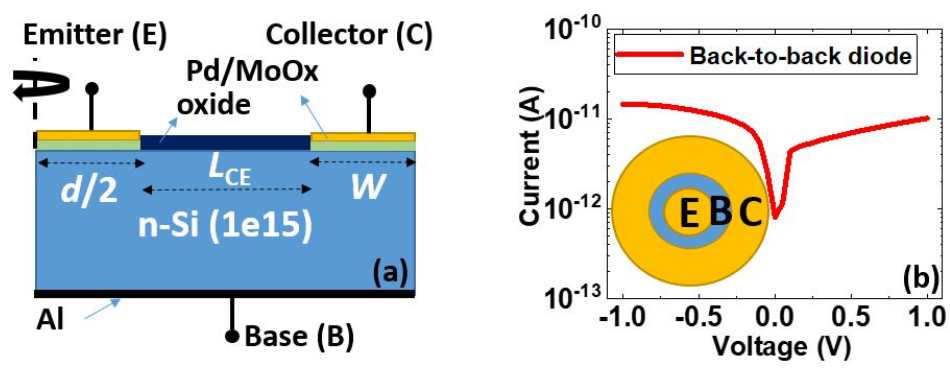

Figure 4.11: (a) Schematic cross-section of the experimental and TCAD simulated device used for bipolar transistor measurements with an inner circle of diameter $\mathrm{d}=100 \mu \mathrm{m}$ as the emitter (E) electrode and outer ring with width $W=50 \mu \mathrm{m}$ as the collector $(\mathrm{C})$ electrode for two different spacings $\mathrm{L}_{\mathrm{CE}}$ of $1 \mu \mathrm{m}$ and $2 \mu \mathrm{m}$, respectively. (b) I - V characteristics of back-to-back diodes measured between the top emitter and collector electrodes. Inset: Schematic top view of the device used for bipolar transistor measurements.

First the two-terminal back-to-back diode characteristics between the collector and emitter electrode was checked as shown in Fig. 4.11(b). The observed low leakage current between the two electrodes rules out the possibility of any unwanted surface conduction path between the emitter and collector.

The bipolar transistor measurements were performed on devices with two different spacings $\left(\mathrm{L}_{\mathrm{CE}}\right)$ between the emitter and collector electrodes i.e. $\mathrm{L}_{\mathrm{CE}}=1 \mu \mathrm{m}$ and $\mathrm{L}_{\mathrm{CE}}=2 \mu \mathrm{m}$. Fig. 4.12(a) shows the Gummel plot for the device with $\mathrm{L}_{\mathrm{CE}}=1 \mu \mathrm{m}$ where a significant collector current $\left(\mathrm{I}_{\mathrm{C}}\right)$ comparable to the base current level, $\mathrm{I}_{\mathrm{B}}$, is obtained at $\mathrm{V}_{\mathrm{CB}}=0 \mathrm{~V}$. The measured $I_{C} \sim I_{B}$ confirms that the improved emitter efficiency of the EB junction as compared to that of conventional Schottky counterparts. The control of the EB junction over the $\mathrm{I}_{C}$ as expected in the bipolar transistor is also clearly visible in the output $\left(\mathrm{I}_{\mathrm{C}}-\mathrm{V}_{\mathrm{CE}}\right)$ characteristics of the same device as shown in Fig. 4.12(b).

The $\mathrm{I}_{\mathrm{C}}$ further increases while $\mathrm{I}_{\mathrm{B}}$ remains unchanged when the reverse bias at the $C B$ junction was raised to $-1 \mathrm{~V}$ which results in the current gain, $\beta=I_{C} / I_{B}>1$. The increased $I_{C}$ and its non-ideal $(n>1)$ behavior for $V_{C B}=$ $-1 \mathrm{~V}$ is attributed to the widening of the depletion width at the $\mathrm{CB}$ junction which eventually results in the punch-through effect, i.e. the depletion regions formed at the EB and CB Schottky junctions gradually start to merge in the gap region between the collector and emitter electrodes. The punch-through effect is also visible in the output characteristics where a low output resistance can be seen. However, for the specified spacing $\mathrm{L}_{\mathrm{CE}}$ and barrier height $\left(\phi_{\mathrm{b}}\right)$, the punch-through effect is not enough to fully eliminate the potential barrier for holes as there is control of $I_{B}$ over $I_{C}$ as expected for the bipolar transistor.

The $\beta$ becomes less than 1 and the observed punch-through effect is practically reduced when the $\mathrm{L}_{C E}$ is increased to $2 \mu \mathrm{m}$ as can be seen in the 

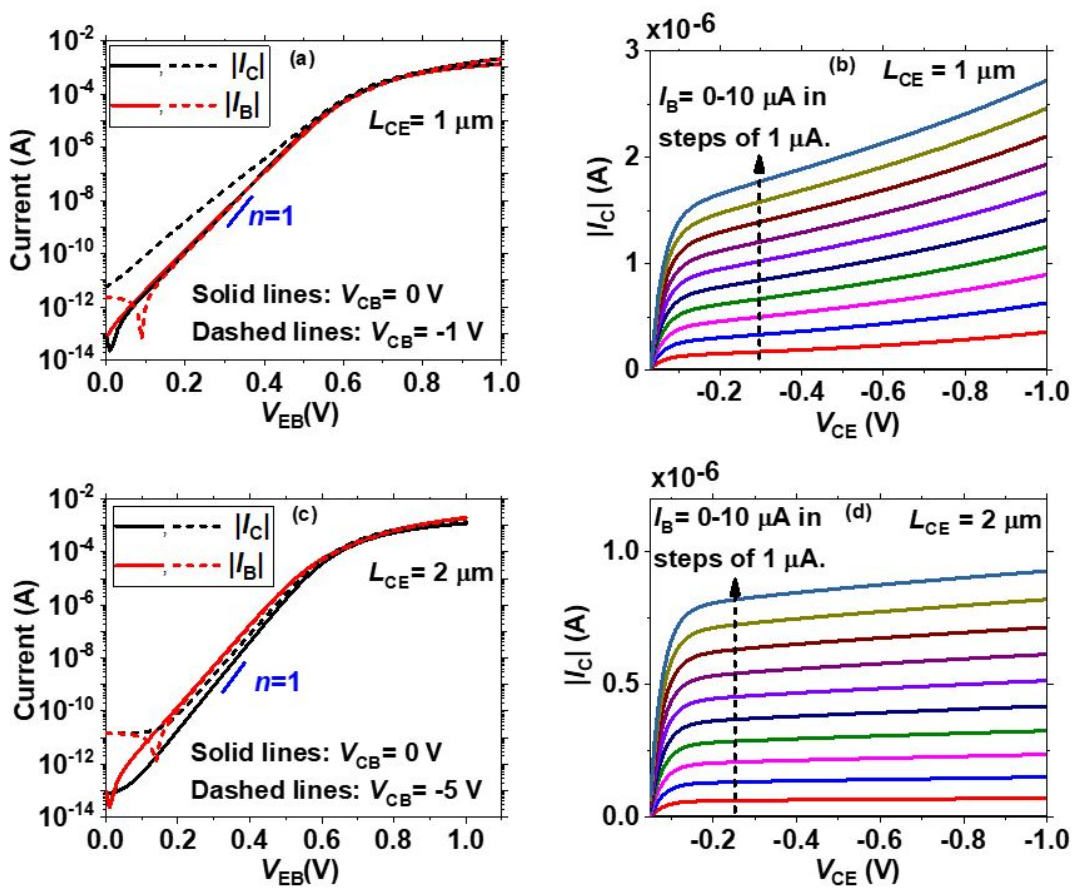

Figure 4.12: Bipolar transistor measurements of the fabricated device. Gummel plot (left) and output characteristics (right) for (a) and (b) $\mathrm{L}_{\mathrm{CE}}=1 \mu \mathrm{m}$, (c) and (d) $\mathrm{L}_{\mathrm{CE}}=2$ $\mu \mathrm{m}$. Blue solid line: ideality factor $\mathrm{n}=1$ for reference.

Gummel plot in Fig. 4.12(c) and the output characteristics in Fig. 4.12(d).

To further illustrate the working of the SBT, TCAD simulations on the same geometry as shown in Fig. 4.11(a) were performed. The top CE electrodes were modeled as Schottky contacts with the identical $\phi_{\mathrm{b}}$ and a fixed (default) [98] surface recombination velocity $\left(S_{\mathrm{n}}=2.5 \times 10^{6} \mathrm{~cm} / \mathrm{s}\right)$. The base electrode was assumed to be an ohmic (neutral) contact. The $\phi_{\mathrm{b}}$ of the top electrodes was then systematically varied. The hole inversion charge density below the collector and emitter electrodes increases with $\phi_{\mathrm{b}}$ as can be observed from the relative position of the Fermi level from the valence band in the energy band diagram in Fig. 4.13(a). Fig. 4.13(a) also shows that the substrate depletion or punch-through effect in the CE gap region increases with $\phi_{\mathrm{b}}$ at the electrodes. However, for $\phi_{\mathrm{b}}$ beyond which the inversion layer effect becomes important, as shown in Fig. 4.8, the effective barrier for holes and depletion width does not change anymore with $\phi_{\mathrm{b}}$. Further, the punch-through effect even at maximum depletion width is not severe and therefore will not affect the bipolar operation, since the barrier to the hole injection is still sufficiently high as required for the base-controlled collector current. Fig. 4.13(b) re-iterates this point by illustrating the operation of the SBT where the minority (hole) injection into the reverse biased $\mathrm{CB}$ junction can be increased by applying a small forward bias at the EB junction which lowers the barrier there. 

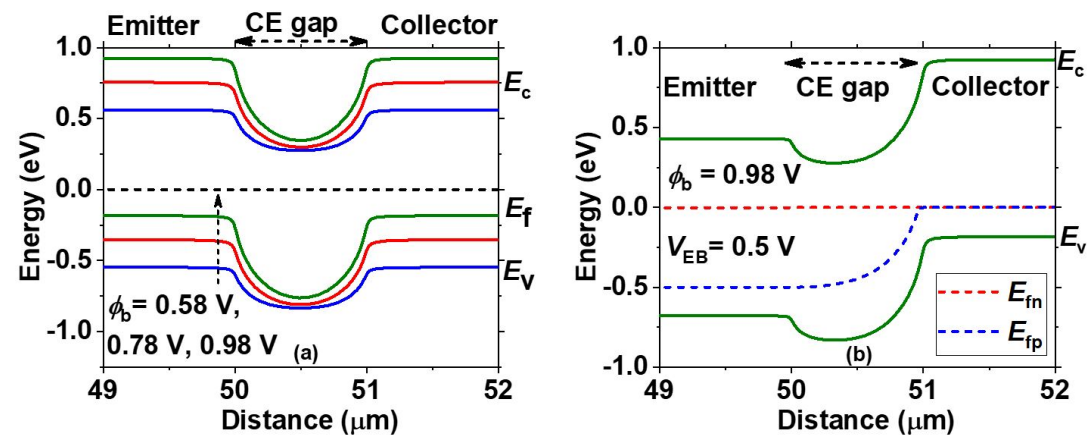

Figure 4.13: Simulated energy band diagram for the bipolar device shown in Fig. 4.11(a), with $\mathrm{L}_{\mathrm{CE}}=1 \mu \mathrm{m}$ in the lateral direction $20 \mathrm{~nm}$ below the top electrodes; (a) at thermal equilibrium for 3 different $\phi_{\mathrm{b}}: 0.58 \mathrm{~V}, 0.78 \mathrm{~V}$ and $0.98 \mathrm{~V}$, and (b) for $\phi_{\mathrm{b}}=0.98 \mathrm{~V}$ at $\mathrm{V}_{\mathrm{EB}}=0.5 \mathrm{~V}$ and $\mathrm{V}_{\mathrm{CB}}=0 \mathrm{~V}$.

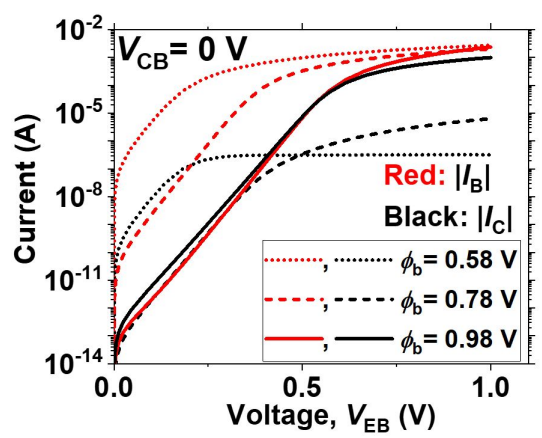

Figure 4.14: Simulated Gummel plots for varying $\phi_{\mathrm{b}}$ at the CE electrodes with $\mathrm{L}_{\mathrm{CE}}=1 \mu \mathrm{m}$, shown for $\phi_{\mathrm{b}}=0.58 \mathrm{~V}, \phi_{\mathrm{b}}=0.78 \mathrm{~V}$ and $\phi_{\mathrm{b}}=0.98 \mathrm{~V}$.

The simulated Gummel plots in Fig. 4.14 further elucidate the role of $\phi_{\mathrm{b}}$ in determining the emitter efficiency of the contacts. The $\mathrm{I}_{\mathrm{B}}$ (shown in red) is determined by the TE of majority carriers (electrons) and therefore decreases exponentially with $\phi_{\mathrm{b}}$ at the emitter electrode. The $\mathrm{I}_{\mathrm{C}}$ on the other hand, for a sufficiently high $\phi_{\mathrm{b}}$, is determined by the minority carrier (hole) injection at the EB junction which is governed by the Gummel number of the base region. Therefore $\mathrm{I}_{\mathrm{C}}$ remains practically unaffected with varying $\phi_{\mathrm{b}}$ as shown in Fig. 4.14 for $\phi_{\mathrm{b}}=0.78 \mathrm{~V}$ and $\phi_{\mathrm{b}}=0.98 \mathrm{~V}$ except at high forward biases where the series resistance becomes important. The relatively small discrepancy in the $\mathrm{I}_{C}$ in the exponential region is attributed to the substrate depletion effect where the effective Gummel number of the base decreases with $\phi_{\mathrm{b}}$ as also illustrated in Fig. 4.13(a). For low $\phi_{\mathrm{b}}=0.58$ $\mathrm{V}, \mathrm{I}_{\mathrm{C}}$ is determined by the reverse bias current of the CB junction as it is higher than the corresponding hole injection level of the forward biased EB junction. The $\beta$ increased to 15 when $\phi_{\mathrm{b}}$ was further raised to an order of $\mathrm{Si}$ bandgap i.e. $1.12 \mathrm{~V}$. In general, the improved emitter efficiency, and therefore higher $\beta$, with increasing $\phi_{\mathrm{b}}$ for the SBT device is a result of the 

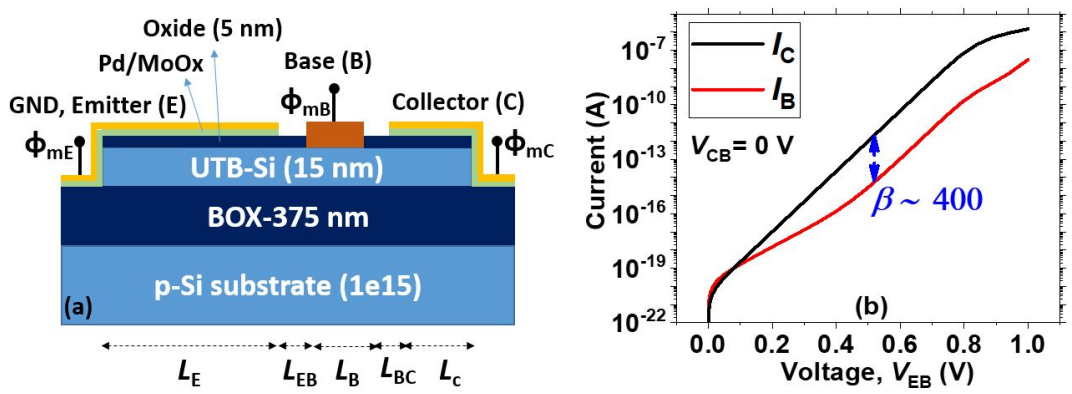

Figure 4.15: (a) Schematic cross-section of the proposed SOI based lateral pnp bipolar transistor [62]. (b) TCAD simulated Gummel plot of the same device. The device dimensions used: $\mathrm{L}_{E}=10 \mu \mathrm{m}, \mathrm{L}_{B}=0.1 \mu \mathrm{m}, \mathrm{L}_{C}=0.4 \mu \mathrm{m}$ and $\mathrm{L}_{E B}=\mathrm{L}_{B C}=0.1 \mu \mathrm{m}$. $\phi_{\mathrm{mE}}=\phi_{\mathrm{mC}}=5.59 \mathrm{eV}$ and $\phi_{\mathrm{mB}}=4.05 \mathrm{eV}$. Mobility: $\mu_{\mathrm{e}}=1417 \mathrm{~cm}^{2} \mathrm{~V}^{-1} \mathrm{~s}^{-1}, \mu_{\mathrm{h}}=470$ $\mathrm{cm}^{2} \mathrm{~V}^{-1} \mathrm{~s}^{-1}$ [98] and SOI layer doping: n-type, $10^{13} \mathrm{~cm}^{-3}$.

efficient suppression of $I_{B}$. Further, the high $\phi_{b}$ and resulting inversion layer ensures an efficient supply of minority carriers for sustaining the $\mathrm{I}_{C}$ till high forward biases and also lowers down the series resistance.

However, it is clear that the chosen device geometry, though interesting for the simple proof-of-concept understanding of the SBT, should be optimized. Alternative device architectures based on UTB SOI, such as the one previously reported by Kumar and Nadda [62] using TCAD simulations, will have more advantages.

To further highlight the potential of such high- $\phi_{\mathrm{b}}$ contacts in UTB SOI, TCAD simulations were performed to show the working of a scaled-down version of the experimentally realized $\mathrm{MoO}_{x}$ based lateral pnp transistor in SOI. A schematic cross-section of the proposed device architecture is shown in Fig. 4.15(a). Here, the experimentally reported interface work function $\left(\phi_{\mathrm{m}}\right)$ of about $5.59 \mathrm{eV}$ [185] was adopted for the $\mathrm{MoO}_{x}$ based contact (on oxide) for the p-type emitter and collector regions with an induced hole concentration of about $10^{18} \mathrm{~cm}^{-3}$. For the n-type base region, an electrode with $\phi_{\mathrm{m}}$ of about $4.05 \mathrm{eV}$ was utilized which results in an induced electron concentration of $4 \times 10^{19} \mathrm{~cm}^{-3}$. A relatively low surface recombination velocity for holes $\left(S_{p}\right)$ of $5 \times 10^{4} \mathrm{~cm} / \mathrm{s}$ was used for the base contact. Note that n-type poly-Si for instance could offer such values for $\phi_{m}$ and $S_{p}$ [186]. The proposed device shows bipolar characteristics with a $\beta$ of about 400, see Fig. 4.15(b). This result indicates that $\mathrm{MoO}_{x}$ based contacts can potentially replace implanted $\mathrm{p}^{+}$regions in the UTB semiconductor for bipolar transistor applications where conventional doping is otherwise challenging. Moreover, such contacts could also be utilized for p-type MOS transistors in undoped UTB semiconductors, specifically narrow bandgap materials. For instance, previously Chuang et al. adopted $\mathrm{MoO}_{x}$ based contacts to realize p-type $\mathrm{MoS}_{2}$ transistors [123]. 


\subsection{Discussion}

In this investigation, the behavior of the $\mathrm{Pd} / \mathrm{MoO}_{x} / \mathrm{n}-\mathrm{Si}$ MnIS diodes, with respect to diode $\mathrm{I}-\mathrm{V}, \mathrm{C}-\mathrm{V}$ and bipolar transistor characteristics, was found to be practically identical to that of the deep-implanted $\mathrm{p}^{+} / \mathrm{n}$ junctions. This is a result of the high minority carrier injection ratio $(\gamma)$ of both types of diodes with a low hole diffusion current and an electron current that is even lower. The presence of a significant inversion layer was confirmed and can to some degree explain low electron injection into the p-type region. In the research on PureB MnIS diodes fabricated at B deposition temperatures below $400^{\circ} \mathrm{C}$, and on the same type of substrates as used in the present study, the inversion layer sheet resistance was lower, $\sim 35 \mathrm{k} \Omega / \square$, and uniform over the wafer when optimal conditions were applied [187]. Both experiments and simulations supported the conjecture that a layer of fixed negative charge at the interface is responsible for the inversion layer in this case.

For the $\mathrm{MoO}_{x}$ diodes, there can be several reasons why they display such low electron current levels. First, the $\mathrm{MoO}_{x}$ is a high work function material, and the simulations performed here substantiate that for barrier heights higher than about $0.9 \mathrm{eV}$, a significant inversion layer will be formed. Second, the passivating nature of $\mathrm{MoO}_{x}$ based contact on $\mathrm{Si}$ $[175,180]$, possibly originating from the observed $\mathrm{SiO}_{x}(\mathrm{Mo})$ interlayer that may be partly insulating and is represented by a low surface recombination velocity, could also play a role in suppressing the electron injection even further. Thirdly, a significant amount of negative fixed charge may also be present in the deposited layer. Unlike thermally grown $\mathrm{SiO}_{2}$ layers that most often contain positive fixed charge near the Si interface, it has been shown before [188] that negative fixed charge may appear in plasmaoxidized Si surfaces. The surplus of oxygen in such layers was proposed to accumulate the negative charge. Similar mechanism may also hold true for the $\mathrm{SiO}_{x}$ interlayer which formed during the $\mathrm{MoO}_{x}$ deposition process.

The partial $\mathrm{SiO}_{x}$ character of the interfacial layer may also be the important feature providing a well-passivated $\mathrm{Si}$ surface. A similar mechanism has been developed and well-documented for $\mathrm{Al}_{2} \mathrm{O}_{3}$ layers that have become popular as passivation layers on n-type c-Si solar cells [189]. As opposed to the $\mathrm{Al}_{2} \mathrm{O}_{3}$, the $\mathrm{MoOx}$ layers are not insulating and therefore they have generated interest as contact layers. The use of $\mathrm{Al}_{2} \mathrm{O}_{3}$ for creating an inversion-layer diode requires contacting the negative-charge-induced inversion layer via implanted regions [170].

From electrical analysis on samples with varying $\mathrm{MoO}_{x}$ thicknesses it can be concluded that only the $\mathrm{MoO}_{x} / \mathrm{Si}$ interface is playing a dominant role in the charge carrier transport of our devices and the bulk properties of $\mathrm{MoO}_{x}$ only contributes to the series resistance. In other work [190], the $\mathrm{MoO}_{x}$ layer was shown to limit the current only at lower voltages while at higher voltages the current was limited by the barrier. Therefore, for 
the situations where the $\mathrm{MoO}_{x}$ layer is not found to limit the current, the charge transport across the $\mathrm{MoO}_{x} / \mathrm{Si}$ interface can be effectively modeled assuming a Schottky electrode with a suitable barrier height and surface recombination velocity [123]. For a more generic treatment of charge transport through a transition metal oxide (TMO) layer, taking into the account the density and the energetic distribution of traps inside its bandgap, the methodology as described by Messmer et al. [182] should be considered.

The bipolar nature of the $\mathrm{MoO}_{x}$ based diode was clearly established from the measured electrical and optical characteristics. Such $\mathrm{MoO}_{\chi}$-based inversion type contacts could also be considered as a possible replacement of doped $\mathrm{p}^{+}$regions for applications where doping is challenging or low temperature processing is required. $\mathrm{MoO}_{x}$-based contacts could offer a novel dopant-free route for realizing (opto-) electronic devices such as field effect transistors (FETs) [123], bipolar transistors, light emitters, photodetectors [191] or even as Schottky gate contacts [192]. Moreover, for creating an n-type inversion region, a lower work function material such as lithium fluoride $\left(\mathrm{LiF}_{\mathrm{x}}\right)$ [163] which has been recently reported as an "electron selective contact" should be further explored. In this way, both p-type and n-type regions can be locally created inside a semiconductor body without using any dopants. However, creating a p-type region is more difficult in case of wide bandgap materials such as $\mathrm{GaN}$ where even the highest reported vacuum work function of $\mathrm{MoO}_{x}$ i.e. $\sim 6.6 \mathrm{eV}$, is not sufficiently high to reach its valence band.

For many of these potential device applications, the high resistivity of the $\mathrm{MoO}_{x}[169,174]$ could be a concern. The contact resistivity, $\rho_{c}$, of the $\mathrm{Pd} / \mathrm{MoO}_{x} / \mathrm{p}-\mathrm{Si}$ stack, with a $\sim 7 \mathrm{~nm}$ thick $\mathrm{MoO}_{x}$ layer, is measured to be around $1.2 \mathrm{~m} \Omega-\mathrm{cm}^{2}$ [169], while this could be an order of magnitude higher on the n-Si interface [174]. In addition to the high $\rho_{\mathrm{c}}$, the key properties of $\mathrm{MoO}_{x}$, such as the work function and conductivity, are unstable upon exposure to ambient and high temperatures [193-196]. These concerns need to be addressed before practical applications become feasible.

With the present quality of $\mathrm{MoO}_{x}$ layers, it is important to cap them in-situ with a suitable metal to prevent exposure to air which otherwise may degrade the work function. The choice of the top electrode metal is also important for efficient carrier transport [182] and for a low contact resistivity. In our work, Pd was used for its reported high work function and relative inert nature at room temperature [123]. A few experiments with Au capping layers were also performed and the results were comparable to those with $\mathrm{Pd}$. Reactive metals such as $\mathrm{Al}$ are less preferred for capping $\mathrm{MoO}_{x}$ as they may reduce the functional $\mathrm{MoO}_{x}$ layer by forming oxides, thereby creating more oxygen vacancies in the film, and consequently lowering the effective work function [196, 197]. 


\subsection{Conclusion}

In this work, MnIS diodes on n-type $\mathrm{Si}$ using $\mathrm{MoO}_{x}$-based contacts were realized. The electrical and light-emitting diode characteristics bore strong functional resemblance to implanted $\mathrm{p}^{+} \mathrm{n}$-Si diodes rather than to conventional Schottky diodes. The presence of a high potential barrier $(>0.90$ $\mathrm{V}$ ) and an hole inversion layer at the $\mathrm{MoO}_{x} / \mathrm{n}$-Si interface was verified with sheet carrier density greater than $\sim 8.6 \times 10^{11} \mathrm{~cm}^{-2}$. The bipolar-mode diode behavior of the $\mathrm{MoO}_{x}$-based MnIS diodes was further underlined by demonstrating a proof-of-concept $\mathrm{Si}$ surface barrier bipolar transistor without any impurity doping of the Si surface.

Theoretically, an inversion layer and efficient suppression of electron injection also appears in very high-barrier Schottky diodes. Simulations that approached the $\mathrm{MoO}_{x} / \mathrm{n}$-Si structure as being a high-barrier Schottky diode could reproduce the experimental findings provided that the surface recombination velocity was set at a relatively low value of $10^{3} \mathrm{~cm} / \mathrm{s}$. This suggests that the surface was passivated which was supported by the presence of $\mathrm{SiO}_{x}(\mathrm{Mo})$ interlayer.

The very high resistivity of the bulk $\mathrm{MoO}_{x}$ material has not deterred possibly attractive implementations for use as solar cell emitter. In contrast, for nanoscale device applications, the associated series resistance could form a serious bottleneck. Therefore, for such applications, thin layers comprising essentially only the interfacial (tunneling) layer should be investigated. Efforts to significantly improve the reproducibility and robustness of the material would also be necessary. If successful, such an MnIS dopant-free hetero-contact processed at a low temperature would be very interesting for fabricating bipolar devices (BJTs, SBTs and LEDs) in Si and other material systems, as well as UTB devices where actual doping is otherwise challenging. 


\title{
ELECTRON-HOLE BILAYER LED
}

\begin{abstract}
In this chapter, a novel switched-mode light-emitting device (LED) based on the electrostatically-induced electron-hole bilayer (EHB) concept has been proposed and investigated using TCAD simulations. The proposed ultra-thin-body (UTB) device works on the principle of formation of EHB channels by applying suitable gate biases during the charging-cycle, and their recombination during a discharging-cycle. TCAD simulations show that continuous switching of the gates in an indium arsenide (InAs) based EHB LED with a $~ 12 \mu$ s time period leads to radiative recombination of the induced charge carriers with a peak internal quantum efficiency (IQE) as high as 92\% and a timeaveraged IQE of $\sim 29 \%$. The proposed concept obviates the need for chemically doped p-n junctions in the UTB device for light-emitting applications. However, when relying on the thermal generation alone as a source of charge carriers in a small undoped semiconductor volume, a narrow bandgap semiconductor (such as InAs) is required for the proposed LED which ultimately limits the switching speed. For wider bandgap materials, highly doped regions on either side of the intrinsic UTB layer in the form of a lateral PIN structure could be employed where switching speed is then not limited by thermal generation. TCAD simulations of a silicon (Si) EHB LED based on such a gated PIN structure shows switching capability in the GHz frequency range making it attractive for SOI based optocoupling applications.
\end{abstract}

This chapter is based on the publication in Solid-State Electronics [57] and EUROSOI-ULIS conference, 2019 [82]. The work was partly pursued as an undergraduate thesis project, undertaken by F. Mema under the author's supervision. 


\subsection{Introduction}

Light-emitting devices based on ultra-thin-body (UTB) semiconductor-oninsulator technology are interesting candidates for inter-chip and intra-chip optical interconnect [198, 199] as well as for sensing applications [200]. In addition to their attractiveness for integration with SOI technology, the UTB based light sources also benefit from their improved efficiency due to quantum confinement, and weak reabsorption of emitted photons inside their ultra-thin active region [201]. To realize such devices, a sharp junction in the UTB semiconductor is one essential requirement which is technologically challenging [2] in conventional chemical doping approaches. In this direction, electrostatic doping (ED) ([46] and references therein) has been widely explored as a potential alternative to chemical doping for UTB devices in Si and emerging semiconductors. Particularly, the electrostatically induced electron-hole bilayer (EHB) [79] is an interesting concept where charge carriers of opposite polarity are simultaneously induced in a dual gate UTB device. Such a concept has been extensively investigated for tunnel field effect transistors (TFETs) applications in various material systems and geometries [79, 202, 203]. A similar concept has also been reported for capacitor-less DRAM memory [204]. However, so far the EHB concept has not been reported for light emission via electroluminescence. In this work [57, 82], a novel light-emitting device (LED) based on the EHB concept in an undoped UTB channel is proposed.

\subsection{Theory and device operation}

The schematic cross-section of the proposed device is shown in Fig. 5.1. The proposed device is a dual gate structure with an UTB semiconductor as a channel material. It comprises two different (low-high) work function metals as top and bottom gate electrodes which are oppositely biased during the device operation. In the simulations the semiconductor body is kept floating. In practice the body can be grounded further away from the active gated device region via a side body contact as discussed in Section 5.4 .

In the dual gate structure as shown in Fig. 5.1 with different top and bottom gate work functions and opposite biasing, electron and hole channels can be simultaneously induced [79]. To describe the work function and bias-induced charge carriers near each gate in such a fully-depleted UTB channel, the following equations (Eqs. (5.1)-(5.2)) for the vertical electric field $\left(E_{s}\right)$ and surface potential $\left(\psi_{\mathrm{sT}, \mathrm{B}}\right)$ at both gate interfaces were derived before $[46,107]$ (refer to appendix A) assuming negligible charge carrier concentration:

$$
E_{\mathrm{s}}=\frac{\left(V_{\mathrm{GT}}-\mathrm{V}_{\mathrm{GB}}\right)-\left(\phi_{\mathrm{mT}}-\phi_{\mathrm{mB}}\right)}{\frac{\varepsilon_{\mathrm{s}}}{\varepsilon_{\mathrm{ox}}}\left(t_{\mathrm{oxT}}+t_{\mathrm{oxB}}\right)+t_{\mathrm{s}}},
$$




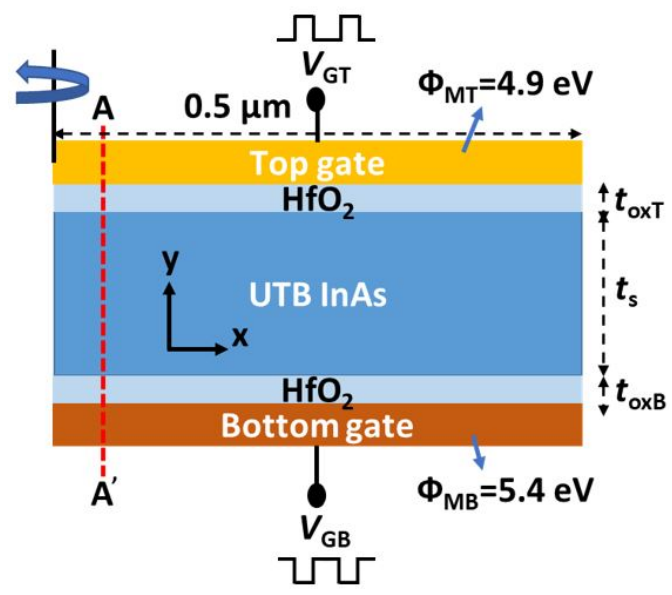

Figure 5.1: Schematic cross-section of the proposed electron-hole bilayer InAs LED.

$$
\psi_{\mathrm{sT}, \mathrm{B}}=\mathrm{V}_{\mathrm{GT}, \mathrm{B}} \mp \frac{\varepsilon_{\mathrm{s}} \mathrm{E}_{\mathrm{s}}}{\mathrm{C}_{\mathrm{oxT}, \mathrm{B}}}-\Delta \phi_{\mathrm{mT}, \mathrm{B}},
$$

where the subscripts $\mathrm{T}$ and $\mathrm{B}$ refer to the top and bottom gate, respectively. $V_{\mathrm{GT}, \mathrm{B}}$ is the gate voltage, $\phi_{\mathrm{mT}, \mathrm{B}}$ is the gate metal work function, $\Delta \phi_{\mathrm{mT}, \mathrm{B}}$ is gate-body work function difference, $\mathrm{C}_{\mathrm{oxT}, \mathrm{B}}$ is the areal oxide capacitance, $t_{\mathrm{ox}}$ and $t_{\mathrm{s}}$ are the oxide and semiconductor thickness, respectively, and $\varepsilon_{\mathrm{ox}}$ and $\varepsilon_{\mathrm{s}}$ are the oxide and semiconductor dielectric constant, respectively.

Eq. (5.1) expresses the field for the EHB formation in the coupled dual gate system. $E_{s}$ also determines the field in the gate oxides, and as a result the maximum bias settings in the EHB device can be determined from this equation. Semi-classically, electrons will be induced near the top gate electrode if $\psi_{\mathrm{sT}}>0$ with a density at the interface $n=n_{i} \exp \left(\psi_{\mathrm{sT}} / u_{T}\right)$ where $n_{i}$ is the intrinsic concentration and $u_{T}$ is the thermal voltage $(=k T / q$, where $k$ is Boltzmann's constant, $T$ is the temperature and $q$ is the elementary charge). Similarly, if $\psi_{\mathrm{SB}}<0$ holes will be induced near the bottom gate electrode with a density at the interface $p=n_{i} \exp \left(-\psi_{\mathrm{sB}} / u_{\mathrm{T}}\right)$. However, since the influence of the mobile charge was neglected for the derivation of Eqs. (5.1)-(5.2), these are therefore not valid for a strong inversion situation. Moreover, quantum effects can modify the carrier densities and their distribution [108]. Further, the effect of interface trap charges can be accounted for as described in [46].

In the proposed EHB structure, which is similar to a parallel plate capacitor, the induced charge carriers remain fixed near their respective gate interfaces in steady state and do not recombine. However, the EHB device becomes electrically active as soon as (one or) both gates are switched between their charged- and discharged-state. During the discharging-cycle, the electron and hole distribution will gradually decay with time via recombination processes. Carrier recombination in a direct bandgap material has 
a high chance to be radiative. The result will be then light emission during a switching transient. Steady light output could therefore be obtained by continuously switching the gates.

InAs is an interesting material in this context because of its narrow and direct bandgap $\left(E_{\mathrm{g}}=0.354 \mathrm{eV}\right.$ at $\left.300 \mathrm{~K}\right)$ which first makes it more suitable for ED [46]. Second, the small $E_{g}$ also results in a higher thermal generation rate of charge carriers which along with Shockley-Read-Hall $(\mathrm{SRH})$ lifetime $\left(\tau_{\mathrm{SRH}}\right)$ determines the switching speed in the proposed device. A simplified expression for time $t_{t h}$ required to thermally generate $n_{0}$ level of carriers from its intrinsic concentration $n_{i}$ can be derived from the continuity equations as (see appendix C):

$$
t_{\text {th }}=\frac{n_{0}}{\frac{n_{i}}{2 \cdot \tau_{\text {SRH }}}+B_{\text {rad }} \cdot n_{i}^{2}}
$$

where $B_{\text {rad }}$ is the radiative recombination coefficient.

Fig. 5.2 shows $t_{t h}$ against $E_{g}$, required to thermally generate $n_{0}=10^{18}$ $\mathrm{cm}^{-3}$ carriers for various direct bandgap semiconductors and $\mathrm{Si}$, calculated using Eq. (5.3), from their respective intrinsic carrier level. For narrow bandgap semiconductors such as $\operatorname{InAs}\left(\tau_{\mathrm{SRH}} \sim 1 \mathrm{~ns}[205]\right), t_{\text {th }}$ is in a few $\mu$ s range while for a wide bandgap material such as $\mathrm{GaN}$, it is in order of $10^{17} \mathrm{~s}$, indicating that the proposed device concept will only work for the former case.

Previously, both an electron [206] and a hole [207] inversion layer was observed in InAs MOS capacitors even at frequencies as high as $1 \mathrm{MHz}$ suggesting a $t_{t h}$ in the $\mu$ s range, which agrees well with Eq. (5.3). Further, an UTB InAs-on-insulator (XOI) technology suitable for realizing the proposed device has been experimentally demonstrated [208, 209].

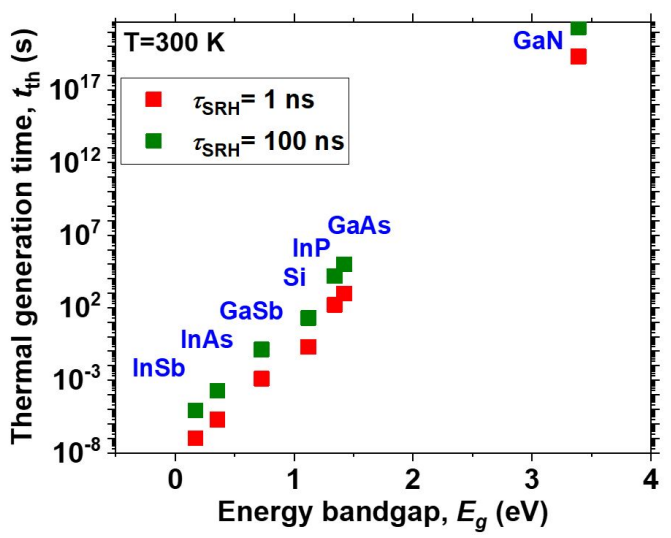

Figure 5.2: Calculated carrier thermal generation time using Eq. (5.3) for various direct bandgap semiconductors (and Si) to reach a carrier concentration of $\sim 10^{18}$ $\mathrm{cm}^{-3}$ from their intrinsic level shown for two different $\tau_{\mathrm{SRH}}$. 
Table 5.1: TCAD simulation parameters for InAs EHB LED at $300 \mathrm{~K}$ [98].

\begin{tabular}{|c|c|}
\hline Parameter & Value \\
\hline InAs bandgap, $\mathrm{E}_{\mathrm{g}}$, electron affinity, $\chi$ & $0.354 \mathrm{eV}, 5.06 \mathrm{eV}$ \\
\hline $\mathrm{HfO}_{2}$ bandgap, $\mathrm{E}_{\mathrm{g}}$, electron affinity, $\chi$ & $5.9 \mathrm{eV}, 2.05 \mathrm{eV}$ \\
\hline Electron effective density of states mass, $\mathrm{m}_{\mathrm{c}}$ & $0.023 \mathrm{~m}_{0}$ \\
\hline Hole effective density of states mass, $\mathrm{m}_{\mathrm{v}}$ & $0.48 \mathrm{~m}_{0}$ \\
\hline Conduction band effective density of states, $\mathrm{N}_{\mathrm{c}}$ & $9.3 \times 10^{16} \mathrm{~cm}^{-3}$ \\
\hline Valence band effective density of states, $\mathrm{N}_{\mathrm{v}}$ & $8.4 \times 10^{18} \mathrm{~cm}^{-3}$ \\
\hline Radiative recombination coefficient, $\mathrm{B}_{\mathrm{rad}}$ & $1.1 \times 10^{-10} \mathrm{~cm}^{3} \mathrm{~s}^{-1}$ \\
\hline SRH lifetime, $\tau_{\mathrm{SRH}}$ & $1 \mathrm{~ns}^{6}$ \\
\hline Auger recombination coefficient & $10^{-30} \mathrm{~cm}^{6} / \mathrm{s}$ \\
\hline Dielectric constant, $\epsilon_{\mathrm{s}}, \epsilon_{\mathrm{ox}}$ & $14 \epsilon_{0}, 22 \epsilon_{0}$ \\
\hline Thickness, $\mathrm{t}_{\mathrm{s}}, \mathrm{t}_{\mathrm{ox}}$ & $25 \mathrm{~nm}, 4 \mathrm{~nm}$ \\
\hline Gate work function, $\phi_{\mathrm{mT}}, \phi_{\mathrm{mB}}$ & $4.9 \mathrm{eV}, 5.4 \mathrm{eV}$ \\
\hline
\end{tabular}

\subsection{Simulation parameters and models}

The proposed device (Fig. 5.1) was investigated via 2-D TCAD simulations with cylindrical symmetry around y-axis using Sentaurus [98], applying Fermi-Dirac statistics with doping [210] and field dependent mobility models [211]. For recombination, standard SRH, Auger and radiative models [98] were included without taking into account any surface recombination at semiconductor-oxide interfaces. Also, the density gradient model [109, 212] was used to account for quantum confinement effects. A low $/$ high work function $\left(\phi_{\mathrm{mT}}=4.9 \mathrm{eV}, \phi_{\mathrm{mB}}=5.4 \mathrm{eV}\right)$ was used for the top/bottom gate for inducing electrons/holes. The chosen gate metal work functions are reasonably symmetric with respect to the mid-gap work function $\left(\phi_{\text {midgap }}=5.18 \mathrm{eV}\right)$ of intrinsic InAs. The InAs body thickness $\left(t_{\mathrm{s}}\right)$ of 25 $\mathrm{nm}$ was chosen to balance the trade-off between the induced charge carrier concentration and their radiative recombination rate. Moreover the chosen thickness is well-outside the range of supercoupling effect $[213,214]$, which otherwise becomes dominant at smaller thicknesses and prevent the co-existence of electron and hole channels. Hafnium dioxide $\left(\mathrm{HfO}_{2}\right)$ with $\mathrm{t}_{\mathrm{oxT} / \mathrm{oxB}}=4 \mathrm{~nm}$ was used for both gate dielectrics which provides a sufficiently high conduction band $\left(\Delta \mathrm{E}_{\mathrm{c}}\right)$ and valence band $\left(\Delta \mathrm{E}_{v}\right)$ discontinuity as to prevent gate leakage (see Fig. 5.3(a)). Ideal interfaces without any traps or fixed charges were assumed. All simulations were performed at 300 K. Table 5.1 summarizes the parameter values used in this work.

\subsection{Device operation: InAs EHB LED}

In UTB devices, the electric field inside the semiconductor body is practically constant (see e.g. Fig. 5.3(a)). The electric field inside the InAs body decreases for a larger thickness and increases as the gate biases are raised to higher voltages as shown in Fig. 5.4. The calculated electric field (Eq. 

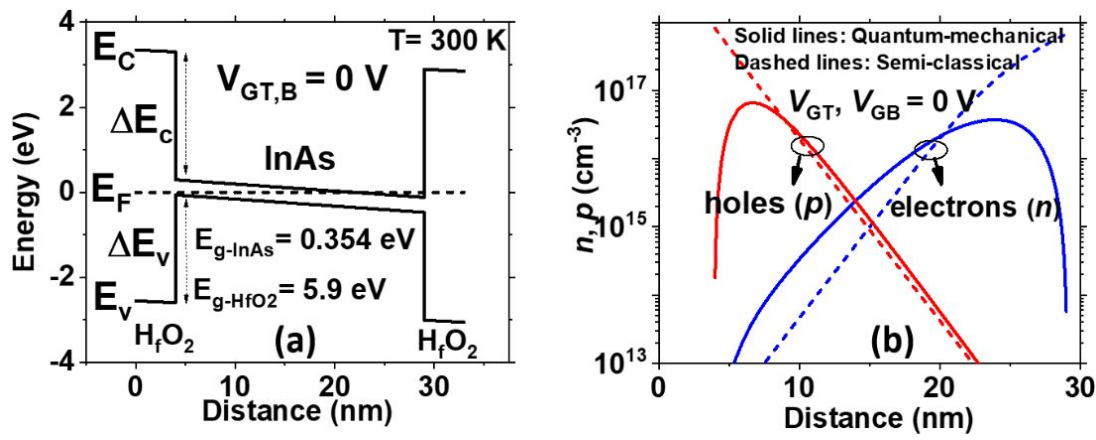

Figure 5.3: TCAD simulations at thermal equilibrium, (a) energy band diagram taken along $\mathrm{AA}^{\prime}$ (see Fig. 5.1). (b) charge carrier profile taken along $\mathrm{AA}^{\prime}$.

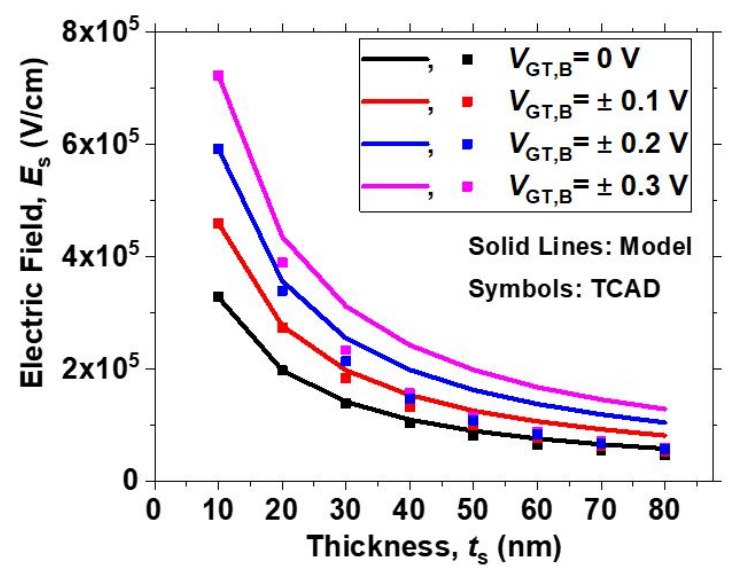

Figure 5.4: Vertical electric field $E_{s}$ in the InAs layer against the thickness $t_{s}$ for a different gate bias. The field has been probed at the middle of the InAs body thickness.

(5.1)) for weak inversion condition, i.e. either small body thicknesses or low gate voltages, is in good agreement with the TCAD simulation results. Some deviation between the model and TCAD is observed for strong inversion condition, i.e., when large body thicknesses $\left(t_{\mathrm{s}} \gtrsim 20 \mathrm{~nm}\right)$ or high gate voltages $\left(\left|\mathrm{V}_{\mathrm{GT}, \mathrm{B}}\right| \gtrsim 0.25 \mathrm{~V}\right)$ are used. This can be explained by the influence of the high charge carrier concentration on the electrostatic potential which is not taken into account in Eq. (5.1).

The charge carrier profiles formed by work function induced ED at thermal equilibrium are shown in Fig. 5.3(b). The induced electron layer is relatively thick compared to the hole layer, as also observed experimentally [206], and is attributed to the relatively small electron effective (density of states) mass, $m_{c}$, which results in a small conduction band effective density of states, $\mathrm{N}_{\mathrm{c}}$. The carrier concentrations can be further increased by applying a small $(\sim 0.75 \mathrm{~V})$ positive (negative) bias at the top (bottom) gate as shown in Fig. 5.5(a)-(b). The situation where a high concentration 

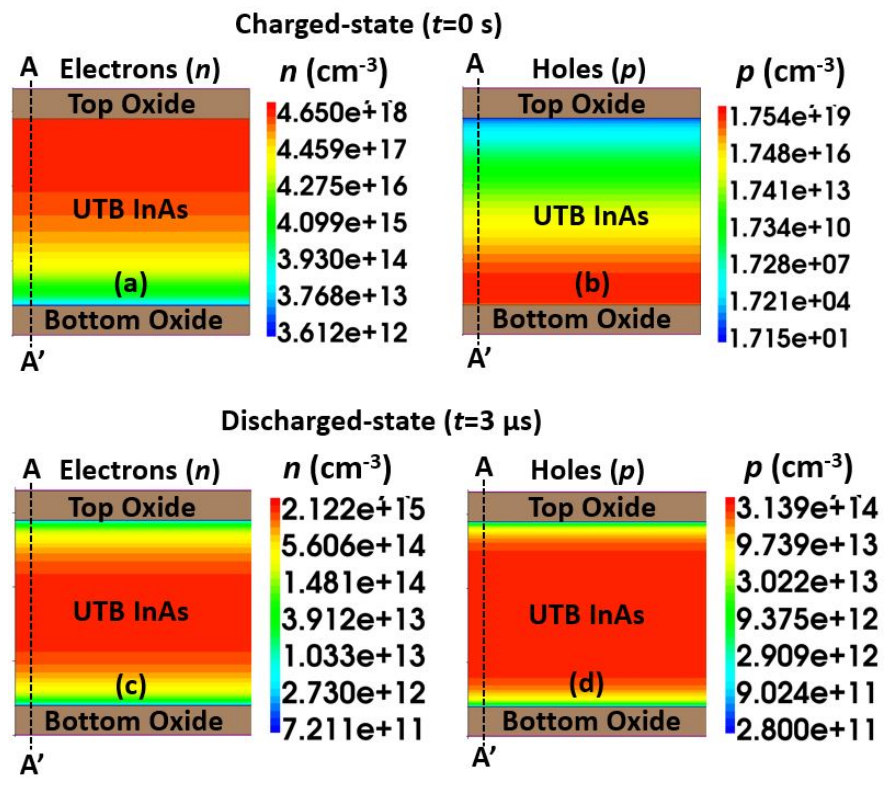

Figure 5.5: Simulated 2-D cross section of induced charge carriers, (a)-(b) at chargedstate $(t=0 \mathrm{~s}),(\mathrm{c})-(\mathrm{d})$ at discharged-state $(\mathrm{t}=3 \mu \mathrm{s})$.

$\left(\sim 10^{18} \mathrm{~cm}^{-3}\right)$ of both electrons and holes are simultaneously induced at opposite gate interfaces is hereafter referred to as charged-state. Since even at thermal equilibrium an EHB has been formed because of the gate-body work function difference, some opposite biasing $\left(\sim\left|\phi_{\mathrm{mT} / \mathrm{B}}-\phi_{\text {midgap }}\right|\right)$ is required to make the body intrinsic. By applying a small $(\sim 0.25 \mathrm{~V})$ negative (positive) bias at the top (bottom) gate, the body becomes intrinsic $\left(\sim 10^{15}\right.$ $\mathrm{cm}^{-3}$ ) as shown in the Fig. 5.5(c)-(d). This situation is hereafter referred to as the discharged-state.

When the EHB is suddenly switched from its charged- to dischargedstate, the charge carriers will decay via recombination processes (both radiative and non-radiative) to reach their final steady state value, see Fig. 5.6. The diffusion and recombination of holes can be observed from their transient density profiles in Fig. 5.6 where a spread in their vertical distribution along with a drop in their peak concentration are visible during the discharging-cycle, even when there is still an opposing electric field. However, electrons are mainly recombining and diffusion is less important, possibly because of a smaller slope ( $\partial \mathrm{n} / \partial \mathrm{y})$ of their vertical distribution profile. Fig. 5.7 shows a radiative recombination rate, $R_{\text {rad, }}$, thus light emission) as high as $\sim 10^{24} \mathrm{~cm}^{-3} \mathrm{~s}^{-1}$ at $0.5 \mu \mathrm{s}$ after the on-set of dischargingcycle.

By continuously switching the gates, as shown in Fig. 5.8, the charge carriers are first thermally generated to form an EHB during the chargingcycle and later recombine during the discharging-cycle. In this way, steady light output via radiative recombination in the form of short $(\sim 0.5 \mu \mathrm{s})$ 


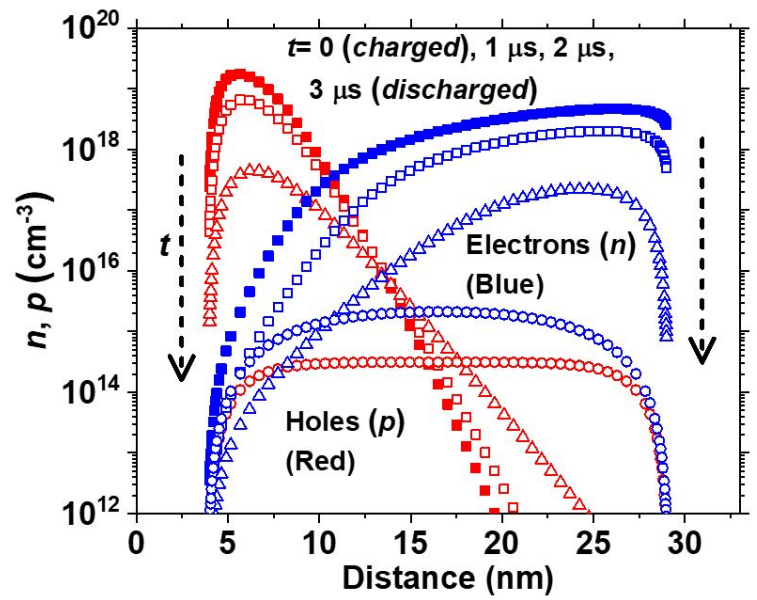

Figure 5.6: Transient electron and hole density (along line $\mathrm{AA}^{\prime}$ ) at various time instances $(t)$ during the discharging-cycle.
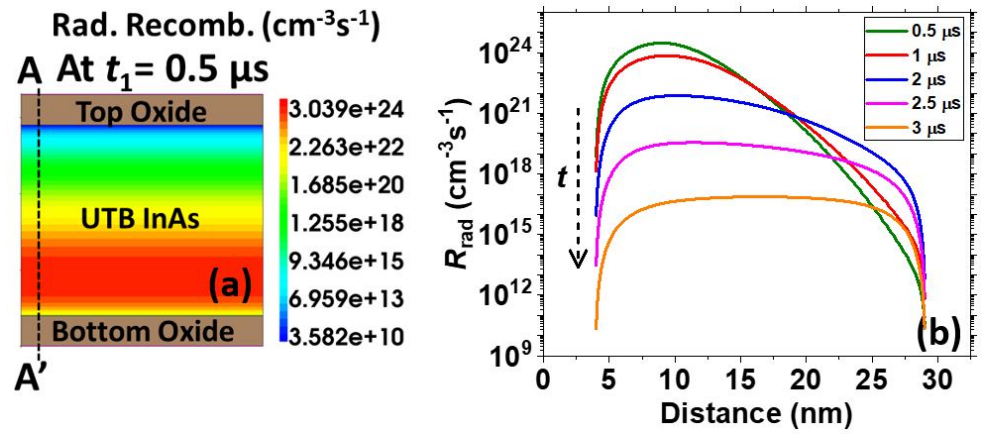

Figure 5.7: (a) 2-D profile of radiative recombination rate at $t_{1}=0.5 \mu$ s after the on-set of the discharging-cycle. (b) Transient radiative recombination rate (along line $\mathrm{AA}^{\prime}$ ) at various time instances $(\mathrm{t})$ during the discharging-cycle.

pulses can be maintained. The pulse width here is taken as the time in which the radiative recombination rate falls by about one order in magnitude. In these transient simulations, the voltage pulse was defined as a piecewise linear function with a rise $/$ fall time $\left(t_{\text {rise } / \text { fall }}=3 \mu \mathrm{s}\right)$, an on-time $\left(t_{\text {on }}=5 \mu \mathrm{s}\right)$ and off time ( $\left.t_{\text {off }}=1 \mu \mathrm{s}\right)$. The chosen $t_{\text {on }}(5 \mu \mathrm{s})$ is sufficiently long for generating charge carriers from their intrinsic value to their high charged-state value.

Moreover, the EHB concentration at the charged-state and consequently $\mathrm{R}_{\text {rad }}$ can be further increased by applying a bias higher than $0.75 \mathrm{~V}$, but that may degrade the dielectric layer over time. Note that the probed electric field in the $\mathrm{HfO}_{2}$ layer at the charged-state $\left(\left|\mathrm{V}_{\mathrm{GT} / \mathrm{B}}\right|=0.75 \mathrm{~V}\right)$ is $\sim 6.5 \times 10^{5}$ $\mathrm{V} / \mathrm{cm}$ which is well below its breakdown value [215]. However, the chosen biasing scheme is not the optimized one as it depends on the type of application. Other biasing schemes can also be explored, such as one involving 


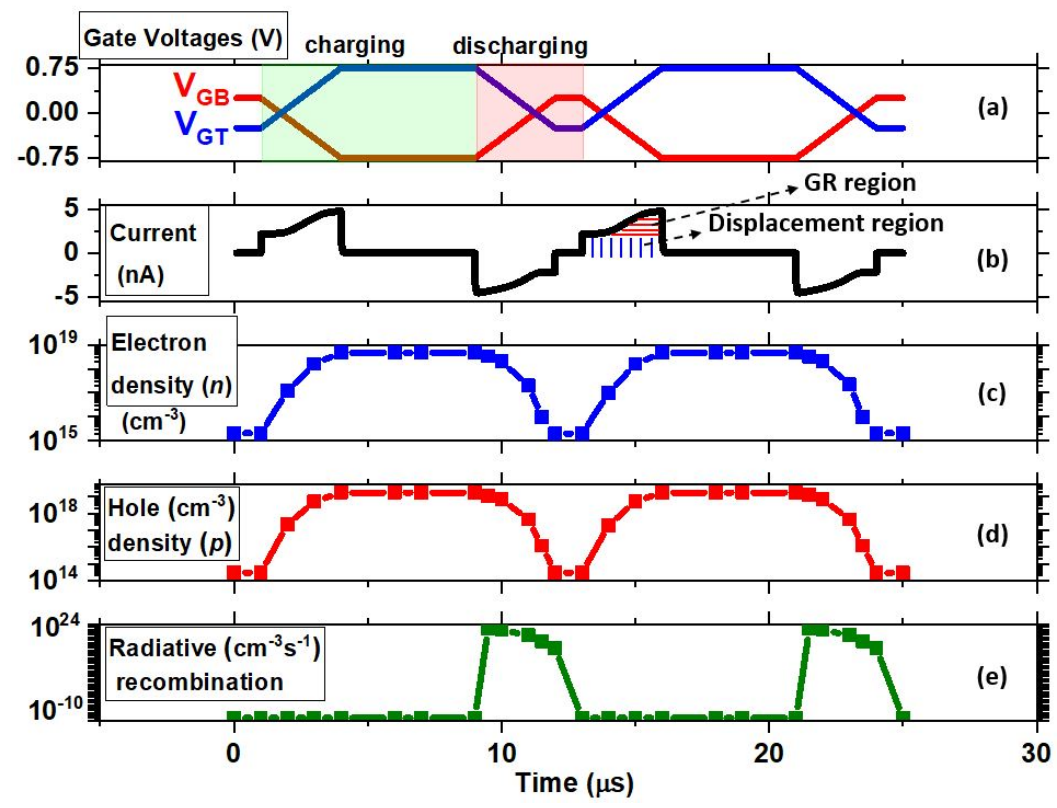

Figure 5.8: Transient simulations of InAs EHB LED operation showing, (a) the gate voltage switching cycles, (b) the device current, (c) the peak electron density near the top gate interface, (d) the peak hole density near the bottom gate interface, (e) the maximum radiative recombination rate in the device. (charged-state $\mathrm{V}_{\mathrm{GT}}=0.75$ $\mathrm{V}$ and $\mathrm{V}_{\mathrm{GB}}=-0.75 \mathrm{~V}$, discharged-state $\mathrm{V}_{\mathrm{GT}}=-0.25 \mathrm{~V}$ and $\mathrm{V}_{\mathrm{GB}}=0.25 \mathrm{~V}$, electrode area, $A_{\mathrm{E}}=7.85 \times 10^{-9} \mathrm{~cm}^{2}$.)

the continuous switching of electron and hole channel positions between the top and bottom gate interfaces by applying higher positive/negative biases. This scheme will be more suitable for a device where both the top and bottom gate consist of the same metal with $\phi_{\mathrm{mT}}=\phi_{\mathrm{mB}} \sim \phi_{\text {midgap }}$.

Further, the role of thermal generation in the proposed device can be analyzed from the current vs time $(\mathrm{I}-\mathrm{t})$ curve shown in Fig. 5.8(b). The switching current here is composed of two parts: the generation/recombination (GR) current and the displacement current. The displacement current is actually a result of the applied voltage switching in the capacitor and is equal to $C \cdot d V / d t$ where $d V / d t$ is constant for the linear voltage ramp and $C$ is the effective capacitance of the dual gate MOS stack which is also constant in the absence of any generation process. The GR current is the measure of carrier generation which in our case is thermal and is affected by parameters such as $n_{\mathrm{i}}, \tau_{\mathrm{SRH}}$ and $B_{\text {rad }}$. The integral of the GR current over time in the $\mathrm{I}-\mathrm{t}$ plot (indicated by the red horizontal lines) in Fig. $5.8(b)$ is equal to the total generated charge in that given time.

In addition, the possible influence of a body side contact on the device operation when placed near the gated region for a ground reference was investigated, and compared that to the previous situation where no such side contact was used. While the device operation remains unaffected, 
the peak $R_{\text {rad }}$ is however reduced ( by one order of magnitude) when a side metal contact with $\phi_{\mathrm{m}} \sim \phi_{\text {midgap }}$ was used. This reduction in $R_{\text {rad }}$ is attributed to the carrier leakage via this side contact. The carrier leakage can be suppressed by using a side contact with either a high or low $\phi_{\mathrm{m}}$ (in reference to $\phi_{\text {midgap }}$ ). Moreover in practice, such contact can be placed further away from the gated region of the device which would further minimize the leakage.

\subsection{Efficiency estimation}

\subsubsection{Internal quantum efficiency (IQE)}

During the discharging-cycle, both radiative and non-radiative recombination processes such as Auger and SRH occur in the active region of the device. The left vertical-axis in Fig. 5.9 shows the contribution of SRH, radiative and Auger processes to the total recombination across the body thickness. The internal quantum efficiency (IQE) is defined as the ratio of the radiative recombination rate $\left(\mathrm{R}_{\mathrm{rad}}\right)$ to the total recombination rate [216]. The right vertical-axis in Fig. 5.9 shows the variation of IQE across the body thickness at different time instances during the discharging-cycle. As can be seen, a peak IQE as high as $\sim 92 \%$ is obtained at $t_{1}=0.5 \mu$ shich gradually drops to $\sim 60 \%$ at $t_{2}=1 \mu$ s after the on-set of discharging-cycle. The integrated time-averaged IQE over one period across the whole device volume is $\sim 29 \%$. Note that both the peak radiative recombination rate and IQE are shifted towards the bottom gate. This is attributed to the asymmetric distribution of electrons and holes, and thus the $p \cdot n$ product (see Fig. 5.6) in the UTB, due to their unequal effective masses.

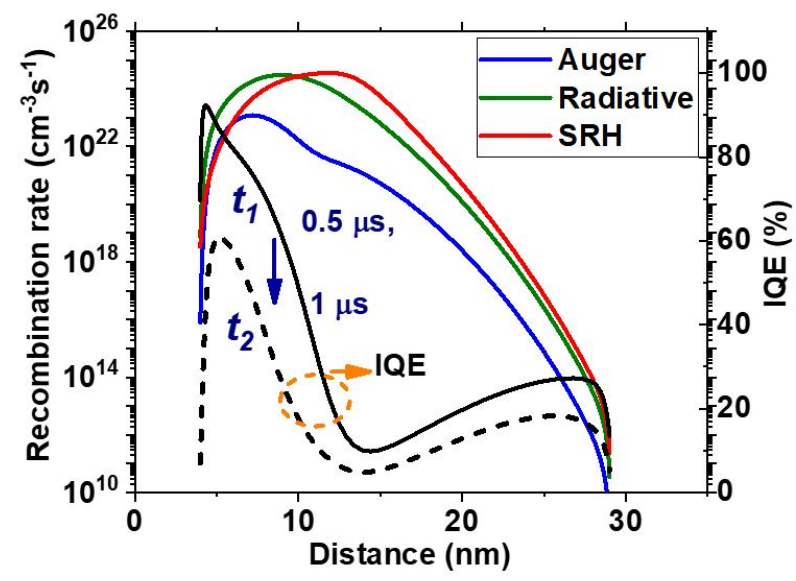

Figure 5.9: Simulated recombination processes across $\mathrm{AA}^{\prime}$ at $t_{1}=0.5 \mu$ s after the on-set of discharging-cycle (left vertical-axis). The IQE profile across $\mathrm{AA}^{\prime}$ (right vertical-axis) at $t_{1}=0.5 \mu$ s and $t_{2}=1 \mu \mathrm{s}$. 


\subsubsection{Power efficiency}

From Fig. 5.8, the power efficiency ( $\eta_{\text {power }}$ ) of the proposed device, which is the ratio of optical power (energy) output to total electrical power (energy) input, was also estimated. The dynamic energy dissipation during the charging and discharging of a parallel plate capacitor can be expressed as [217]:

$$
\mathrm{E}_{\text {elec }}=\mathrm{QV}=\mathrm{V} \int_{0}^{\mathrm{T}}|\mathrm{I}| \mathrm{dt},
$$

where $\mathrm{Q}$ is the total charge generated in one cycle, $\mathrm{V}$ is the total voltage swing, I is the current and T is the cycle time. Similarly, the optical energy output during the switching cycle can be expressed as:

$$
E_{\text {opt }}=V \int_{0}^{T} I_{o p t} d t=q V \int_{0}^{T} R_{i-r a d} d t,
$$

where $R_{i-\text { rad }}=\iiint R_{\text {rad }} d v$ is the integrated radiative recombination rate across the whole device volume.

Therefore,

$$
\eta_{\text {power }}=\frac{q \int_{0}^{\mathrm{T}} R_{\mathrm{i}-\mathrm{rad}} d t}{\int_{0}^{\mathrm{T}}|\mathrm{I}| \mathrm{dt}} \times 100 \% .
$$

Using Eq. (5.6), the maximum $\eta_{\text {power }} \sim 15 \%$ for our device was estimated. For this calculation the light extraction efficiency of our LED was assumed to be $100 \%$ which in practice is difficult to achieve in such a dual gate UTB structure. Measures such as the use of transparent electrodes or electrodes with regular openings should be considered for efficient light extraction.

\subsection{Device optimization: Body thickness}

The effect of the InAs thickness $t_{s}$ on the induced charge carrier concentrations and the radiative recombination rate was also checked. As shown in Fig. 5.10(a), the induced electron and hole densities initially increase with the thickness as described by Eqs. (5.1)-(5.2), valid for a fully depleted semiconductor body where the two opposite gate interfaces are electrostatically coupled. The electrostatic coupling gradually reduces for larger thicknesses, and as a result, the carrier concentration at their respective gate interfaces increases. For even larger body thicknesses, the charge carrier concentrations gradually saturate to the value which is mainly determined by the potential of their respective gate only. 

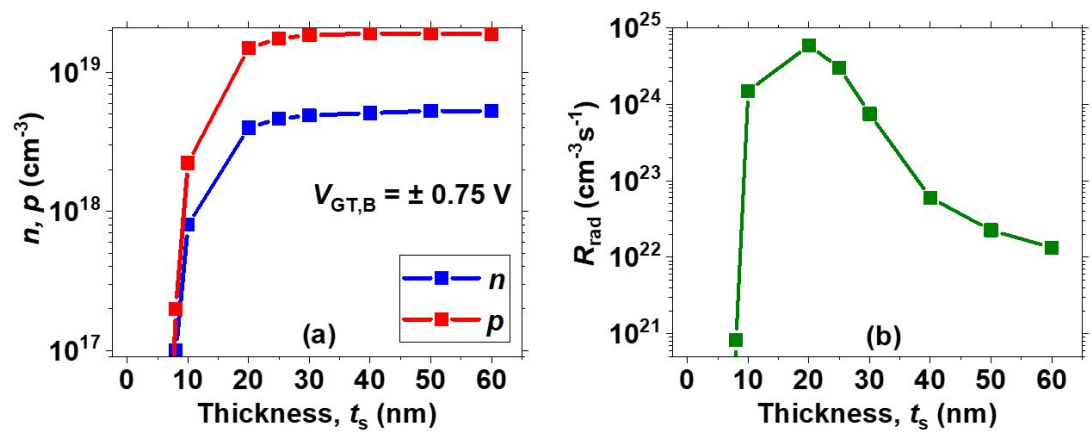

Figure 5.10: TCAD simulation for a varying InAs thickness $t_{s}$, (a) induced peak electron $(n)$ and hole $(p)$ concentrations at the charged-state condition at their respective gate interfaces, (b) peak radiative recombination rate $\left(R_{\mathrm{rad}}\right)$ where $R_{\mathrm{rad}}$ is noted at $t_{1}=0.5 \mu$ s after the on-set of discharging-cycle.

For thicknesses below $10 \mathrm{~nm}$, the sharp drop in carrier density is attributed to a very high electric field and resulting supercoupling effect [213] Note that in our simulated dual gate structure, which features symmetric gate work functions and equal gate oxide thicknesses, the two gates are of equal strength and consequently the possibility of one gate winning over the other is rather small in the supercoupling regime. This however may not occur in e.g. SOI based devices where generally the top gate with a smaller gate oxide thickness controls the channel in that regime.

The radiative recombination rate on the other hand first increases with the thickness as the induced carrier concentrations increase and thereafter decreases as the spatial separation between the induced electron and hole layer increases as shown in Fig. 5.10(b). The maximum radiative recombination rate is at around $t_{\mathrm{s}}=20 \mathrm{~nm}$ which also justifies our initial choice for the thickness value used for the device simulations.

Moreover, as discussed, the supercoupling effect can be important in such dual gate UTB devices. Therefore, it is worthwhile to further estimate the critical thickness, $t_{\mathrm{s}}^{*}[213,214]$ below which such effect becomes important. The simplified expression for $t_{\mathrm{s}}^{*}$ as derived before in [213], assuming a strong inversion situation at the top gate $\left(\mathrm{V}_{\mathrm{GT}}>\mathrm{V}_{\mathrm{TT}}\right)$, can be expressed as:

$$
t_{\mathrm{s}}^{*}=u_{\mathrm{T}} \frac{\epsilon_{\mathrm{s}}}{\mathrm{C}_{\mathrm{oxB}}\left(\mathrm{V}_{\mathrm{FBB}}-\mathrm{V}_{\mathrm{GB}}\right)} \ln \left[\frac{\mathrm{N}_{\mathrm{a}} \mathrm{C}_{\mathrm{oxT}} \mathrm{C}_{\mathrm{oxB}}\left(\mathrm{V}_{\mathrm{FBB}}-\mathrm{V}_{\mathrm{GB}}\right)}{\mathrm{q} \epsilon_{\mathrm{s}} \mathrm{n}_{\mathrm{i}}^{2}}\right]
$$

where $\mathrm{C}_{\mathrm{oxB}}, \mathrm{V}_{\mathrm{GB}}$ and $\mathrm{V}_{\mathrm{FBB}}$ are the areal oxide capacitance, applied voltage and flatband voltage at the bottom gate respectively, $C_{\text {oxT }}, V_{\mathrm{GT}}$ and $V_{\mathrm{TT}}$ are the areal oxide capacitance, applied voltage and threshold voltage at the top gate respectively and $\mathrm{N}_{\mathrm{a}}$ is the body doping density. To estimate $t_{\mathrm{s}}^{*}$ for our proposed device structure, Eq. (5.7) was used, which is applicable to long channel devices as in this work but ignores quantum effects. For this estimation in case of our unintenionally doped device, $\mathrm{N}_{\mathrm{a}}$ was replaced by the intrinsic carrier concentration $\left(n_{\mathrm{i}} \sim 10^{15} \mathrm{~cm}^{-3}\right)$. At $\mathrm{V}_{\mathrm{GB}}=-0.75 \mathrm{~V}$, 
the calculated $t_{\mathrm{s}}^{*}=0.8 \mathrm{~nm}$, which is in fair agreement with our TCAD simulations (including quantum effects) where the supercoupling effect was evident for $t_{s}$ below $5 \mathrm{~nm}$. Therefore, supercoupling is not much of a concern for our proposed dual gate structure with a symmetric top and bottom gate. It could however be an issue if a thicker bottom gate oxide is used. For example, the calculated $t_{\mathrm{s}}^{*}$ is about $26 \mathrm{~nm}$ for a $200 \mathrm{~nm}$ thick back oxide for the same $\mathrm{V}_{\mathrm{GB}}$.

As an application note, the proposed In(Ga)As based EHB LED can be monolithically integrated with an InAs photodiode, which could be attractive for a wide range of applications in the mid-infrared spectral region $(2-10 \mu \mathrm{m})$ such as gas sensing and environmental monitoring [218, 219].

\subsection{Diode-mode operation}

In addition to the proposed dual gate EHB device shown in Fig 5.1, an alternative device structure, as shown in Fig. 5.11(a), was investigated where the InAs body is now directly sandwiched between two metal electrodes (with $\phi_{\mathrm{mT}}=4.9 \mathrm{eV}$ and $\phi_{\mathrm{mB}}=5.4 \mathrm{eV}$ ) without any gate oxide in between. For these metal-semiconductor interfaces, a surface recombination velocity of $S_{n}=2.5 \times 10^{6} \mathrm{~cm} / \mathrm{s}$ and $S_{p}=1.9 \times 10^{6} \mathrm{~cm} / \mathrm{s}$ [98] were used in the simulations. All other simulation parameters were kept the same as summarized in Table 5.1 .

The simulated energy band diagram for such a device at thermal equilibrium is shown in Fig. 5.11(b). Two Schotttky barriers have been formed with different barrier heights $\left(\phi_{\mathrm{b}}\right)$ for electrons and holes at the opposite interfaces. Moreover, the work function induced electron and hole layers are also present at the top and bottom interface respectively. The charge transport in such a device can be considered to be equivalent to a series connection of an n-type and p-type Schottky diode with each having a different $\phi_{\mathrm{b}}$. The diode with a lower $\phi_{\mathrm{b}}$ would then determine the current. The top interface determines the hole current while the bottom interface determines the electron current in the device, both via thermionic emission. The thermionic emission of charge carriers is also evident from the forward bias band diagram (see e.g. Fig. 5.11(c)) where both electron and hole quasi Fermi levels remain flat throughout the whole body [1]. The simulated $\mathrm{I}-\mathrm{V}$ characteristics of the device is shown in Fig. 5.11(d). Since $\phi_{\mathrm{bn}}<$ $\phi_{\mathrm{bp}}$, the electron component will determine the total device current at low injection with an ideality factor, $n$, of unity. At high injection, where $n=2$, the electron and hole current are comparable.

In such diode-mode operation, a lower steady-state IQE of $\sim 16 \%$ at $V_{d}=0.5 \mathrm{~V}$ was obtained which is expected to deteriorate further in the presence of tunneling processes, which were not accounted for. The observed lower IQE in the diode-mode operation is attributed to the enhanced $\mathrm{SRH}$ recombination rate in the presence of a more uniform distribution 

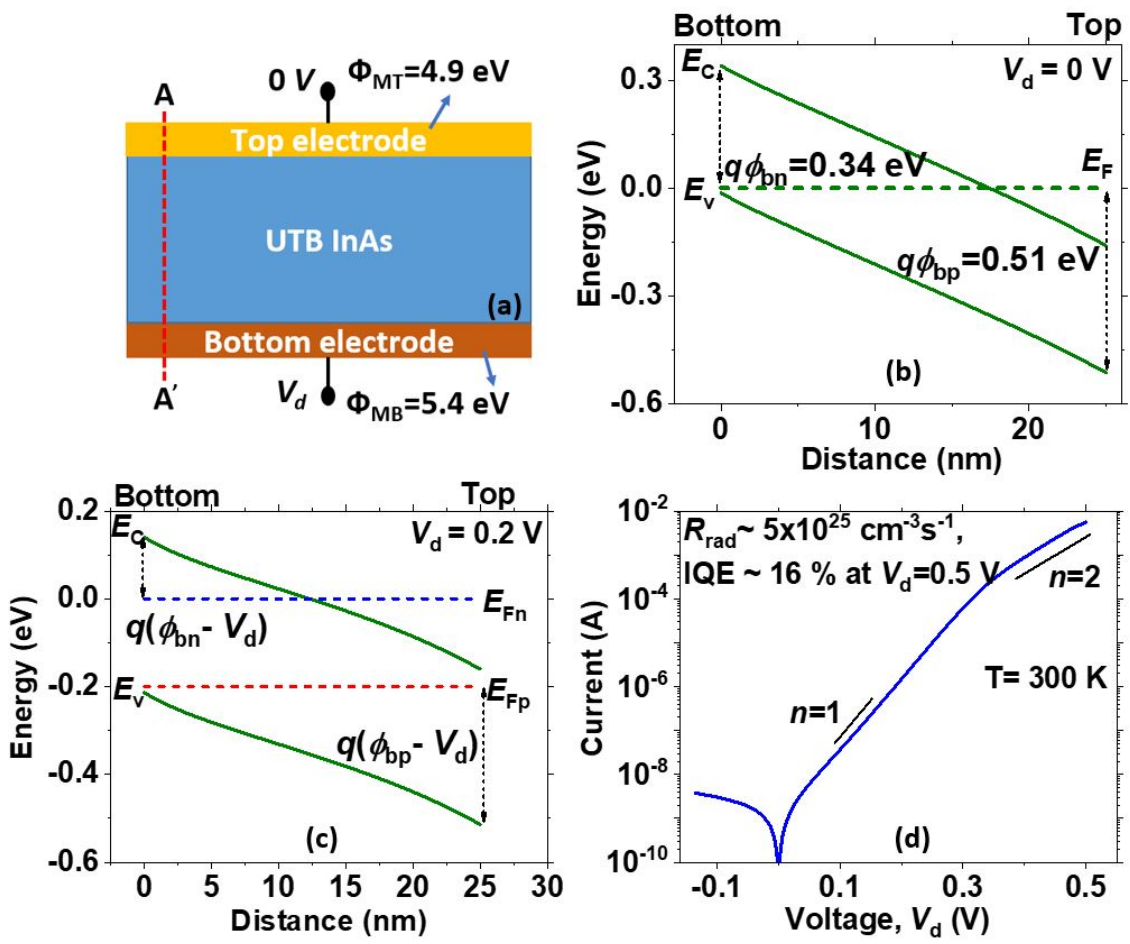

Figure 5.11: InAs EHB device without gate oxides, (a) schematic cross-section, (b) simulated energy band diagram across $\mathrm{AA}^{\prime}$ at thermal equilibrium and (c) at forward bias, $V_{d}=0.2 \mathrm{~V}$ and $(\mathrm{d})$ simulated I-V characteristics of the same device. Electrode area, $A_{E}=7.85 \times 10^{-9} \mathrm{~cm}^{2}$.

( $p \sim n \sim$ constant) of charge carriers across the body thickness [158]. This is not the case for the switched-mode operation in the dual gate EHB device where the carrier concentrations gradually drop from their respective gate interfaces leading to $p \neq n$ and therefore a lower SRH rate (for the same SRH lifetime).

Furthermore, in practice, direct metal-semiconductor junctions are prone to the Fermi-level pinning effect [176] and therefore obtaining such Schottky barriers and resulting work function induced EHB is rather difficult. Particularly, in InAs, it has been reported that the Fermi level is normally pinned above the conduction band minimum [220]. In that respect, the proposed dual gate InAs EHB device based on the switchedmode operation is more practical as it is less prone to Fermi level pinning. Moreover it even shows higher IQE.

\subsection{Si EHB LED}

The EHB LED concept for a wider bandgap material than InAs, such as $\mathrm{Si}$, with a relatively low thermal generation rate, was also investigated. For such a material, the source of charge carriers in the form of highly 


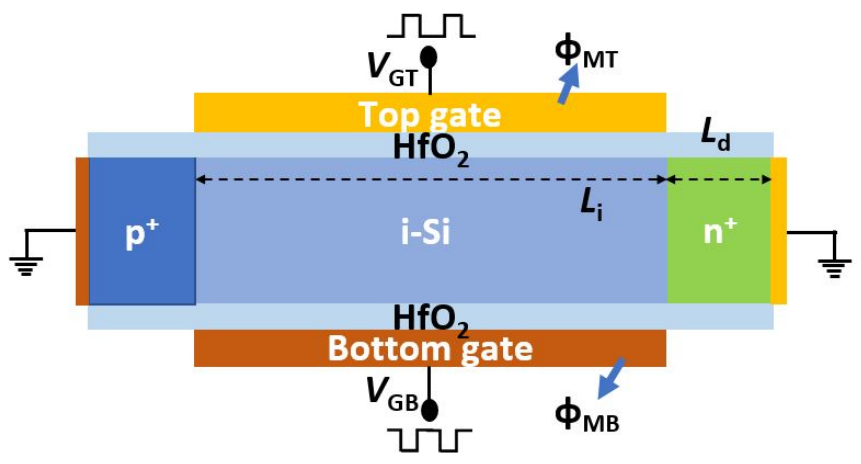

Figure 5.12: Schematic cross section of the Si EHB LED with additional highly doped $\left(\sim 10^{20} \mathrm{~cm}^{-3}\right)$ regions on either side of the UTB intrinsic-Si body. Here, the silicon thickness $t_{s}=12 \mathrm{~nm}, \mathrm{~L}_{\mathrm{i}}=0.5 \mu \mathrm{m}, \mathrm{L}_{\mathrm{d}}=0.1 \mu \mathrm{m}, \mathrm{t}_{\mathrm{oxT} / \mathrm{B}}=4 \mathrm{~nm}, \phi_{\mathrm{MT}}=4.1 \mathrm{eV}$ and $\phi_{\mathrm{MB}}=5.1 \mathrm{eV}$. For Si, SRH lifetimes, $\tau_{\mathrm{n}}=10^{-5} \mathrm{~s}$ and $\tau_{\mathrm{p}}=3 \times 10^{-6} \mathrm{~s}[98]$ and $\mathrm{B}_{\mathrm{rad}}=10^{-14}$ $\mathrm{cm}^{3} \mathrm{~s}^{-1}[221]$ were used.

doped p-type and n-type region on either sides of the intrinsic body, as also originally proposed for the EHB TFET [79], or side metal contacts with a high surface generation velocity should be adopted.

Fig. 5.12 shows the conceptual device architecture for the Si EHB LED with an additional doped region on either side. First, the switched-mode operation of this gated Si EHB device is discussed. During its operation, both gate interfaces are switched between their charged- and dischargedstate by applying the voltage pulses while keeping the side contacts to $\mathrm{p}^{+}$ and $\mathrm{n}^{+}$regions grounded all the time, as shown in Fig. 5.12. The proposed device is capable of switching in the GHz frequency range, see Fig. 5.13

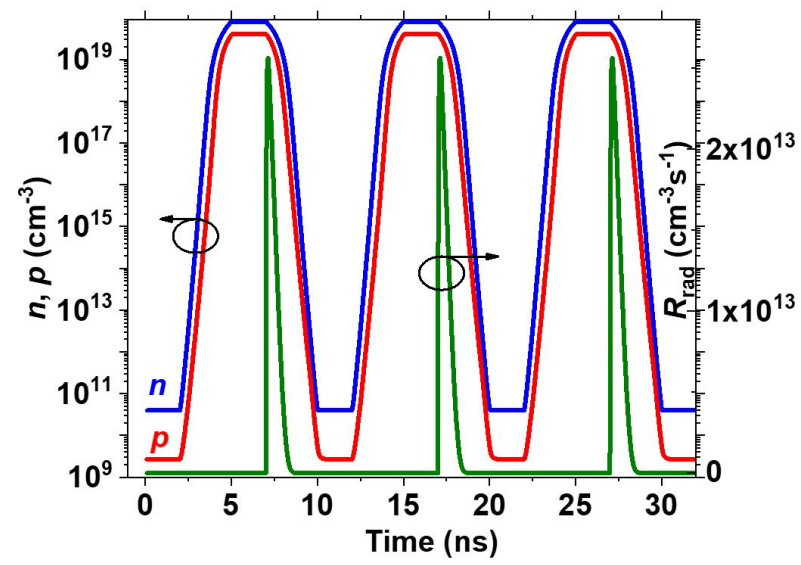

Figure 5.13: Simulated switched-mode operation of the Si EHB LED in ns time scale showing the peak electron and hole density at the opposite gate interfaces (left vertical-axis) and the maximum radiative recombination rate in the device (right vertical-axis). (charged-state $\mathrm{V}_{\mathrm{GT}}=1 \mathrm{~V}$ and $\mathrm{V}_{\mathrm{GB}}=-1 \mathrm{~V}$, discharged-state $\mathrm{V}_{\mathrm{GT}}=-0.5 \mathrm{~V}$ and $V_{G B}=0.5 V, t_{\text {on } / \text { off }}=2 \mathrm{~ns}, t_{\text {rise } / \text { fall }}=3 \mathrm{~ns}$, gate electrode area, $A_{E}=5 \times 10^{-9} \mathrm{~cm}^{2}$.) 
where the formation and recombination of EHB occurs in ns time period. The Si EHB emits low intensity $\left(R_{\mathrm{rad}} \sim 2.5 \times 10^{13} \mathrm{~cm}^{-3} \mathrm{~s}^{-1}\right) \mathrm{ns}$ light pulses with an IQE of about 10 $-3 \%$.

Next, the performance of this Si EHB device is compared with a conventional Si PIN diode of the same geometry as shown in Fig. 5.12 but without any provision of the top and bottom gate. The forward biased Si PIN diode shows a much higher light intensity compared to its EHB counterpart, with an IQE $\sim 10^{-2} \%$. However, GHz switching in SOI PIN LEDs is challenging as discussed before in [222]. In this respect, the proposed Si EHB LED could be more attractive for an SOI based opto-coupling application platform for its high speed switching capabilities.

Furthermore, the device efficiency of the Si EHB LED can be improved if the recombination at the $\mathrm{p}^{+}$and $\mathrm{n}^{+}$regions are effectively suppressed [201]. One way of achieving this is by physically separating the gated EHB regions from the heavily doped regions with an i-Si spacer region. However, the i-Si spacer region would then limit the device speed. So, again there is a trade-off between device speed and its efficiency [222] Moreover, the carrier transit time through the intrinsic region also affects the device operation when it becomes comparable to the carrier lifetime for a longer diode length $\left(\mathrm{L}_{\mathrm{i}}\right)$ and therefore the diode length also needs to be optimized for efficient device operation.

\subsection{Conclusion}

A novel switched-mode light emitting device based on the electrostatically induced EHB concept has been proposed. TCAD simulations show that the InAs based device is capable of switching in the $\mu$ s range to emit light pulses of wavelengths corresponding to its effective bandgap. Monolithic integration of such an optical device along with (opto-)electronic devices in a UTB III-V on insulator (XOI) technology could be interesting for applications such as on-chip communication and sensing. The proposed device is more suitable for narrow bandgap materials since switching speed is limited by the bandgap through the thermal generation rate. In addition, the EHB LED concept is interesting for wider bandgap materials such as $\mathrm{Si}$ in a gated PIN configuration, where high speed switching in the GHz range is possible, which could be explored for SOI based optocoupling applications. 


\title{
POLYCRYSTALLINE GAN/P-SI HETEROJUNCTION DIODE
}

\begin{abstract}
In this chapter, the charge carrier transport and electroluminescence (EL) in thin-film polycrystalline (poly-) GaN/p-Si heterojunction diodes realized using a plasma enhanced atomic layer deposition (PEALD) process are studied. The fabricated poly-GaN/p-Si diode with a native oxide at the interface showed a rectifying behavior $\left(\mathrm{I}_{\text {on }} / \mathrm{I}_{\text {off }}\right.$ ratio $\sim 10^{3}$ at $\pm 3 \mathrm{~V}$ ) with current-voltage characteristics reaching an ideality factor $n$ of $\sim 5.17$. The areal $\left(J_{a}\right)$ and peripheral $\left(J_{p}\right)$ components of the current density were extracted and their temperature dependencies were studied. The space charge limited current (SCLC) in the presence of traps is identified as the dominant carrier transport mechanism for $\mathrm{J}_{\mathrm{a}}$ in forward bias. An effective trap density of $4.6 \times 10^{17} / \mathrm{cm}^{3}$ at a trap energy level of $0.13 \mathrm{eV}$ below the $\mathrm{GaN}$ conduction band minimum was estimated by analyzing $\mathrm{J}_{\mathrm{a}}$. Other basic electrical properties of the material such as free carrier concentration, density of states in the conduction band, electronic mobility and dielectric relaxation time were also determined from the current-voltage analysis in the SCLC regime. Further, infrared EL corresponding to the Si bandgap was observed from the fabricated diodes. The observed EL intensity from the $\mathrm{GaN} / \mathrm{p}$-Si heterojunction diode is $\sim 3$ orders of magnitude higher compared to that of the conventional Si only counterpart. The enhanced infrared light emission is attributed to the improved injector efficiency of the GaN/Si diode because of the wide bandgap of the poly-GaN layer and resulting band discontinuity at the $\mathrm{GaN} / \mathrm{Si}$ interface.
\end{abstract}

This chapter is based on the publication in Journal of Applied Physics [42]. 


\subsection{Introduction}

Gallium Nitride $(\mathrm{GaN})$ is an attractive semiconductor for both electronic and optoelectronic applications because of its wide and direct bandgap, high breakdown field and stability at high temperature [18-20]. The relatively high substrate cost for GaN based devices may be tackled with $\mathrm{GaN}$-on-Si substrates. These additionally allow the monolithic integration of $\mathrm{GaN}$ devices with Si platform technology [21-23]. However, when monocrystalline $\mathrm{GaN}$ is to be grown on silicon, a several-micrometer thick buffer layer is required [21, 24] which makes it difficult to form a platform technology combining silicon devices with $\mathrm{GaN}$ devices on the same chip. A potential alternative approach is to grow thin-film polycrystalline $\mathrm{GaN}$ on silicon. Compared to thick film heteroepitaxy, polycrystalline films bring important fabrication advantages such as lower cost, a lower bill-of-materials and a lower thermal budget. Still, polycrystalline materials generally do not reach the same quality in electrical performance as their monocrystalline counterparts. It is therefore the purpose of this chapter to investigate electrical properties of polycrystalline GaN grown on monocrystalline silicon substrates.

Among the several approaches to grow thin polycrystalline GaN films, atomic layer deposition (ALD) is an attractive solution for applications where excellent wafer-level uniformity, a critical layer thickness control is required, and complex device topologies are involved [36, 37]. An additional advantage of ALD is that it allows to reduce the deposition temperature of many processes [38]. Recently, ALD grown GaN devices such as thin-film transistors have been successfully demonstrated [39-41] with a reasonable performance considering their polycrystalline nature. However so far there have been no reports on vertical devices based on ALD-GaN-on-Si heterojunctions. With this motivation, ALD grown thinfilm polycrystalline GaN/Si heterojunction diodes were realized and their charge carrier transport and optical properties have been investigated in this chapter.

\subsection{Experimental details}

A (111) oriented p-type Si wafer with a background doping concentration of $10^{15} \mathrm{~cm}^{-3}$ was used to fabricate the GaN/p-Si heterojunction diodes ${ }^{1}$. The native oxide present on the surface of the Si wafer was not removed for this experiment. A thin layer of polycrystalline $\mathrm{GaN}$ on $\mathrm{Si}$ substrate was deposited using a plasma-enhanced ALD process, in a remote-plasma Picosun R-200 reactor. The precursors were trimethylgallium (TMG) for Ga and ammonia $\left(\mathrm{NH}_{3}\right)$ for $\mathrm{N}$. The depositions were done at $400{ }^{\circ} \mathrm{C}$ tempera-

\footnotetext{
${ }^{1}$ The poly-GaN thin-film ALD growth, device fabrication and material characterization reported in this chapter were performed by S. Banerjee [223] as part of the joint research project. The chapter though is mainly focused on electrical aspects.
} 

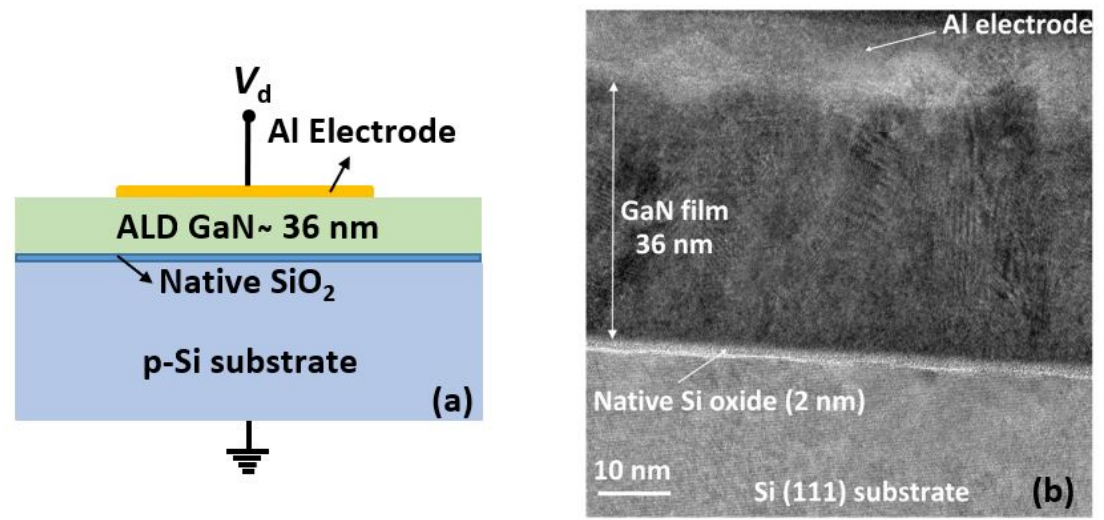

Figure 6.1: (a) Schematic cross-section of the experimental Al/GaN/p-Si diode with circular electrode (area $A_{E}$ ) of diameter $d=100 \mu \mathrm{m}$, (b) Cross-section TEM image of the fabricated device $[42,223]$.

ture. The growth of the layers were monitored in real-time using an in-situ spectroscopic ellipsometer (J.A. Woollam Co. M-2000, operating between 245.8 - $1688.5 \mathrm{~nm}$ wavelength). All polycrystalline GaN samples studied in this chapter were of $36 \pm 3 \mathrm{~nm}$ thickness. For comprehensive account of poly-GaN thin film ALD process, please refer to [223].

Post deposition, the GaN layers were characterized by X-ray photoelectron spectroscopy (XPS, Phi Quantera SXM) for determining the layer composition and by $\theta-2 \theta$-and grazing incidence angle $X$-ray diffraction (GIXRD, Panalytical X'Pert Powder) for determining the layer crystallinity. The layer thicknesses that were obtained from the ellipsometer in-situ monitoring were additionally verified by a transmission electron microscope (TEM, Philips CM300ST-FEG).

$\mathrm{GaN} / \mathrm{Si}$ heterojunction diodes with $\mathrm{Al}$ metal electrodes (i.e., $\mathrm{Al}$ as a top electrode) were then fabricated using a standard photolithography process. The schematic of experimentally realized device is shown in Fig. 6.1(a). All electrical measurements were performed using a Keithley 4200 semiconductor parameter analyser that was equipped with a temperaturecontrolled chuck. The bottom of the Si substrate was utilized as the back contact. Optical measurements were performed using a cooled InGaAs detector based camera (XEVA-320 from Xenics) for capturing the IR micrographs. For measuring the emission spectra, an Avaspec UV-Vis/NIR spectrometer from Avantes was used. Further, an off-chip Si photodiode [156] was utilized for photocurrent measurements.

\subsection{Material characterization of the $\mathrm{GaN}$ layers}

The deposition of polycrystalline (poly-) GaN layers was monitored in real time using in-situ spectroscopic ellipsometry (SE). The layers had a growth 
rate of $\sim 0.095 \mathrm{~nm} /$ cycle. Since the layer thicknesses were extracted using an optical model [224], it was necessary to validate the obtained thickness values. This was done with the help of TEM imaging (Fig. 6.1(b)). Both SE and TEM revealed similar thickness values, i.e., $\sim 36 \mathrm{~nm}$ within an accuracy of $\pm 3 \mathrm{~nm}$.

The TEM image of Fig. 6.1(b) shows the cross-section of the fabricated device (i.e. Al/GaN/native oxide/Si). The image reveals vertical (columnar) growth of $\mathrm{GaN}$ layer at several regions. By performing the fast Fourier transform (FFT) at multiple regions in the GaN layer, d-spacing values typical to (002) planes of hexagonal (wurtzitic) GaN were obtained. Other wurtzitic crystal planes such as (100) and (101) were also observed in small amounts, indicating the polycrystalline nature of the material.

The polycrystalline structure was also reconfirmed with the help of a GIXRD scan (not shown) taken at an incidence angle of $1^{\circ}$. The diffractogram confirmed the wurtzitic structure of the layer and also shows a strong (002) peak, reflecting the abundance of this crystal plane in the polycrystalline layer. The average grain size of the (002) crystal plane from the diffractogram was estimated to be $9.8 \mathrm{~nm}$. This value is slightly higher compared to that observed from TEM images, which showed grain sizes between $\sim 5-9 \mathrm{~nm}$.

The composition and the chemical bonding information in the grown film was determined from XPS, which was equipped with an Al-k $\alpha$ monochromatic X-ray source. The presence of N-Ga bond was confirmed by XPS analysis of the grown poly-GaN films. Further, impurity levels (i.e. C and O) were found to be low inside the film with oxygen content of about $7 \%$ in bulk of the film and $\sim 15 \%$ at the surface as revealed from depth profiled XPS analysis. Practically, no carbon was detected inside the film. The XPS depth profile analysis also found the film to be N-rich. For more detailed description of poly-GaN material characterization, please refer to [223].

\subsection{Electrical Measurements}

The I - V characteristics of the GaN/p-Si diode with native oxide at 298 $\mathrm{K}$ (Fig. 6.2) show a rectifying behavior with an $\mathrm{I}_{\text {on }} / \mathrm{I}_{\text {off }}$ ratio $\sim 10^{3}$ at \pm 3 V. However the semi-logarithmic I $-\mathrm{V}$ plot shows a high ideality factor $n \sim 5.17$. An ideality factor of much higher than two indicates a physical transport mechanism other than thermionic emission or diffusion $(n=1)$ and space charge region recombination $(n \leqslant 2)$. The GaN layer could limit the conduction by bulk controlled processes such as space charge limited current (SCLC) or Poole-Frenkel mechanism. Alternatively, the conduction could be limited by interface controlled processes at the GaN/Si heterojunction such as thermionic emission or tunneling.

Fig. 6.3 shows a simulated energy band diagram of the GaN/p-Si heterojunction in which Anderson's model or the electron affinity rule was used [98]. Here, the electron-affinity of GaN was assumed to be $3.3 \mathrm{eV}$ 


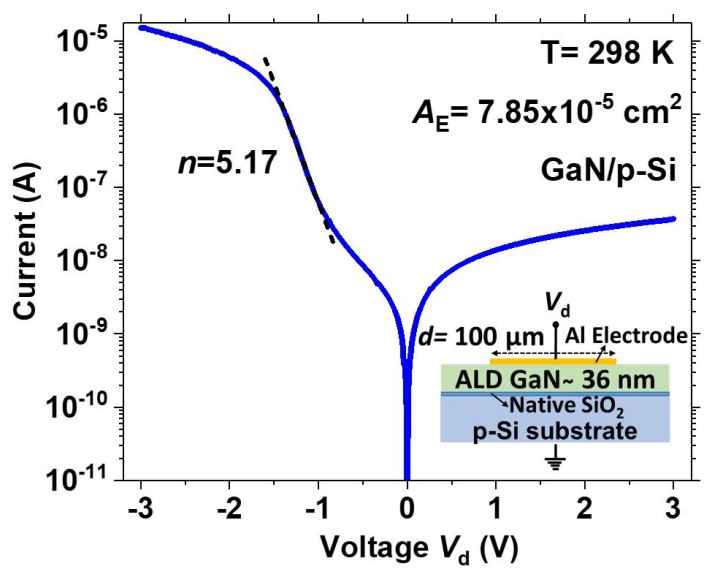

Figure 6.2: I - V characteristics of fabricated GaN/p-Si vertical diode with native oxide at $\mathrm{T}=298 \mathrm{~K}$. Inset: schematic cross-section of the experimental device.

which has been reported before both for crystalline GaN $[225,226]$ and for polycrystalline $\mathrm{GaN}[31,33]$. The large discontinuity in the valence band $\left(\Delta \mathrm{E}_{\mathrm{V}}=1.58 \mathrm{eV}\right)$ as compared to conduction band discontinuity $\left(\Delta \mathrm{E}_{\mathrm{c}}=0.7\right.$ $\mathrm{eV})$ makes it less likely for holes to participate in the conduction process, at least at lower voltages $(<1.58 \mathrm{~V})$. Therefore it is very likely that only electrons will determine the current flow in this device.

To further analyze the charge carriers transport, the areal $\left(\mathrm{J}_{\mathrm{a}}\right)$ from the peripheral $\left(J_{p}\right)$ current density components were first separated via $I-V$ measurement on devices with different perimeter-to-area $(P / A)$ ratio electrodes. The slope of J vs $\mathrm{P} / \mathrm{A}$ curve yields $\mathrm{J}_{\mathrm{p}}$ while the intercept results

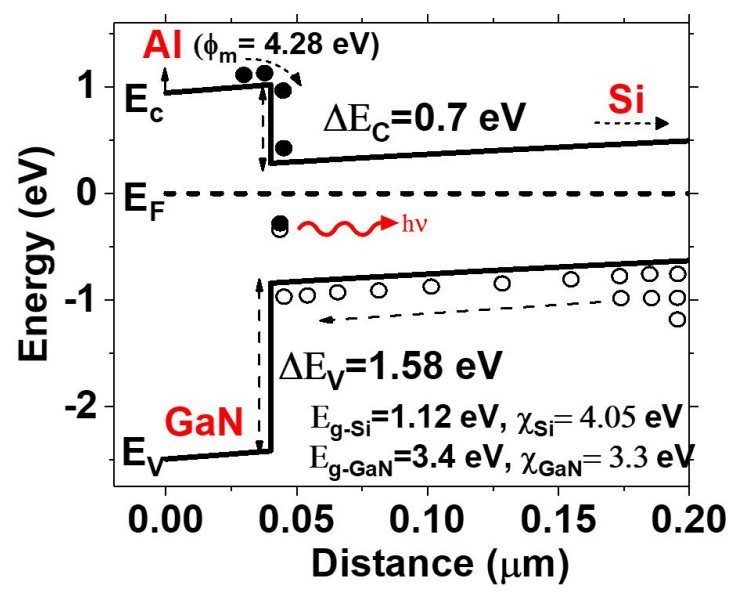

Figure 6.3: TCAD [98] simulated energy band diagram of the GaN/p-Si heterojunction diode at thermal equilibrium. The distance is relative to the top Al electrode. The GaN layer thickness is $40 \mathrm{~nm}$ and thickness of Si substrate is $525 \mu \mathrm{m}$. Filled circles: electrons. Open circles: holes. 

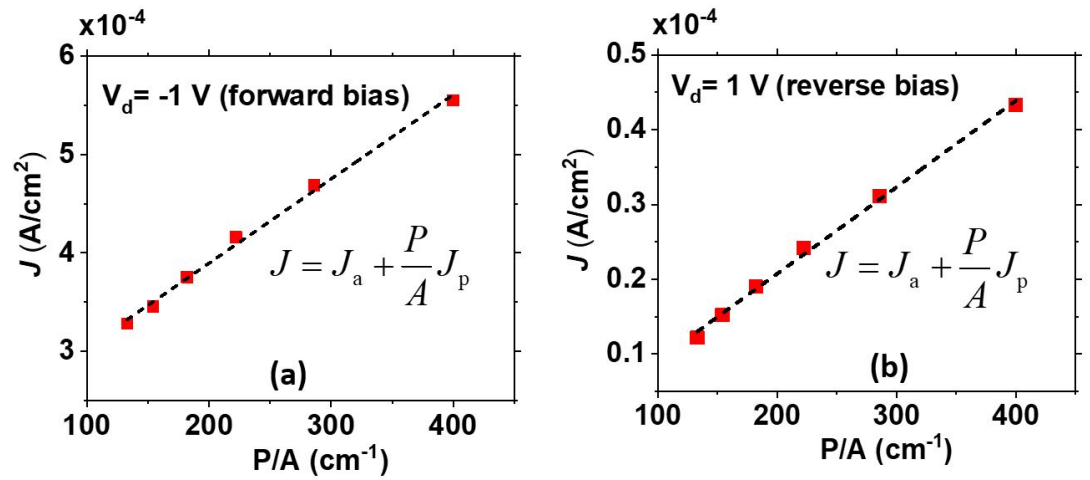

Figure 6.4: Current scaling measurement of devices with a varying perimeter-toarea $(\mathrm{P} / \mathrm{A})$ ratio of the electrode: (a) at forward bias, (b) at reverse bias. J is the total current density, $J_{a}$ and $J_{p}$ are the areal and peripheral current density respectively, $P$ and $A$ are the device perimeter and area respectively.

in $J_{a}$ as shown in Fig. 6.4 for two different voltage levels. Following this approach, $\mathrm{J}_{\mathrm{a}}$ and $\mathrm{J}_{\mathrm{p}}$ were separated for the whole measured voltage range as shown in Fig. 6.5. Both $\mathrm{J}_{\mathrm{a}}$ and $\mathrm{J}_{\mathrm{p}}$ are rectifying in nature.

The extracted $\mathrm{J}_{\mathrm{a}}$ and its high ideality factor could be explained in terms of space charge limited current (SCLC) $[227,228]$ which is a typical conduction mechanism for thin insulating films or lowly doped wide bandgap semiconductors where only a single type of charge carrier participate in the conduction process. This is very likely the case in our devices where no doping was introduced in the GaN layer and conduction is possibly via

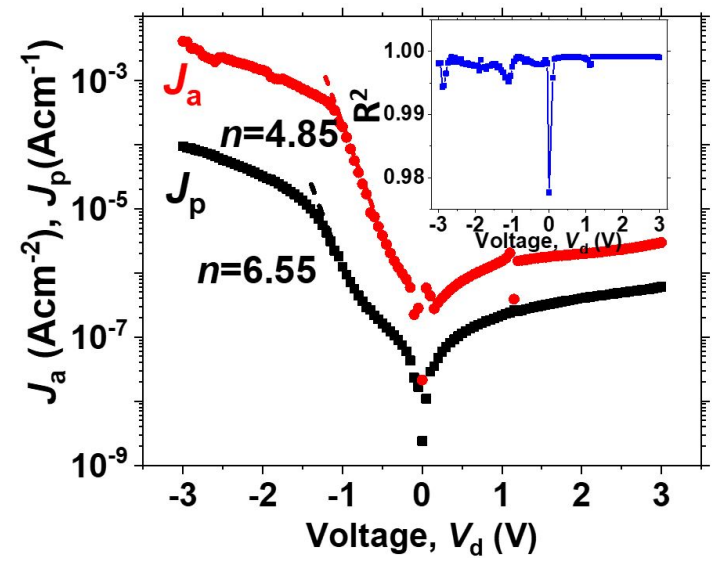

Figure 6.5: Extracted $J_{a}$ and $J_{p}$ via $J-V$ measurement on devices with different perimeter-to-area $(P / A)$ ratio electrodes using the relationship $J=J_{a}+(P / A) J_{p}$. Inset: $R^{2}$ residual from the least-square fitting method. The $R^{2}$ value of $\sim 1$ throughout the measured voltage range indicates good linear fitting and therefore uniform current scaling. 


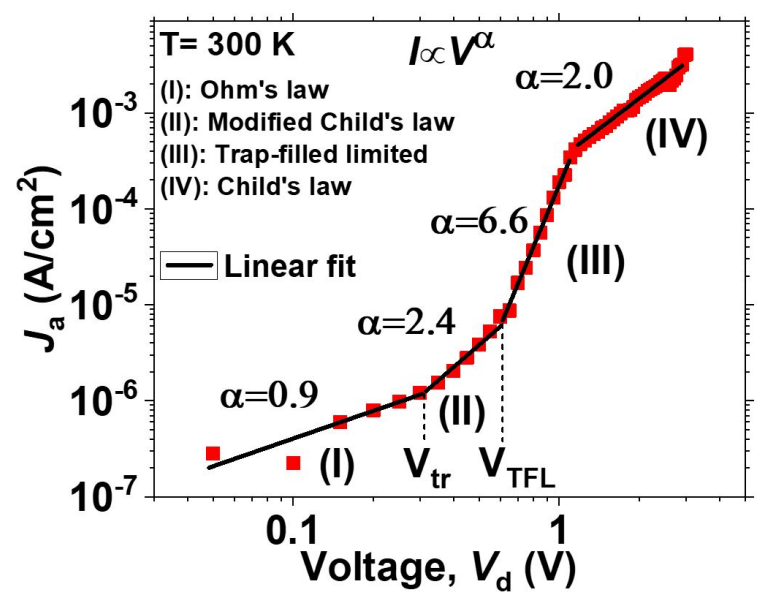

Figure 6.6: $\log (J)-\log (V)$ plot of the extracted $\mathrm{J}_{\mathrm{a}}$ in the forward bias region.

electrons at lower voltages ${ }^{2}$. The trap-mediated SCLC has been reported before for GaN based films and nanostructures [27, 229-231], GaN/Si heterojunctions [232-234] as well as for other wide bandgap semiconductor based heterojunctions such as $\mathrm{ZnO} / \mathrm{Si}[235,236]$ and $\mathrm{ZnO} / \mathrm{GaN}[237]$.

The observed $\mathrm{J}_{\mathrm{a}}-\mathrm{V}$ behavior was analyzed in terms of a single type of carrier (electrons) SCLC model $[227,228]$ in the presence of a single discrete trap level at energy $E_{t}$ below the conduction band edge. The $\log \left(J_{a}\right)-\log (V)$ plot of the GaN/p-Si diode, see Fig. 6.6, shows four linear regimes with different slopes $(\alpha)$ which are separated by a transition voltage, $V_{\text {tr }}$ and a trap-filled limit (TFL) voltage, $V_{\text {TFL }}$. The $J-V$ equations for SCLC in the presence of a single shallow trap level are described as follows [228]:

$$
\begin{gathered}
J_{\text {ohm }}=q n_{0} \mu \frac{V}{d}, \\
J_{\text {Child (trap) }}=\frac{9 \mu \epsilon_{\mathrm{r}} \epsilon_{0} \theta V^{2}}{8 d^{3}}, \\
J_{\text {Child }}=\frac{9 \mu \epsilon_{\mathrm{r}} \epsilon_{0} V^{2}}{8 d^{3}}, \\
V_{\text {tr }}=\frac{8 q n_{0} d^{2}}{9 \epsilon_{\mathrm{r}} \epsilon_{0} \theta}, \\
V_{\mathrm{TFL}}=\frac{q N_{\mathrm{t}} \mathrm{d}^{2}}{2 \epsilon_{\mathrm{r}} \epsilon_{0}}, \\
\theta=\frac{\mathrm{N}_{\mathrm{C}}}{g_{\mathrm{n}} N_{\mathrm{t}}} \exp \left(\frac{E_{\mathrm{C}}-E_{\mathrm{t}}}{k T}\right),
\end{gathered}
$$

${ }^{2}$ The role of device electrical contacts in supplying the required charge carriers for SCLC transport is discussed in chapter 7 . 


$$
\tau_{\mathrm{c}}=\frac{\mathrm{d}^{2}}{\mu \mathrm{V}_{\mathrm{tr}}},
$$

where $q$ is the elementary charge, $k$ is the Boltzmann constant, $\epsilon_{0}$ is the permittivity of vacuum, $d$ is the thickness of the insulator or space charge region, $\epsilon_{\mathrm{r}}$ is the relative permittivity of the medium, $\mu$ is the electron mobility, $\mathrm{n}_{0}$ is the free carrier concentration, $\theta$ is the ratio of free carriers to total carriers, $E_{C}$ is the position of conduction band edge, $E_{t}$ is the trap energy level with respect to $E_{C}, g_{n}$ is the degeneracy of the states in the conduction band $(\sim 2)$ [238], $\mathrm{N}_{C}$ is the conduction band effective density of states, $N_{t}$ is the effective trap density of the medium and $\tau_{c}$ is the carrier transit time.

At low bias $\left(0<\mathrm{V}<\mathrm{V}_{\mathrm{tr}}\right.$, region I), there is ohmic conduction (Eq.(6.1)) with $\alpha \sim 1$ where the thermally generated free carrier density $\left(n_{0}\right)$ is larger than the injected carrier density $(n)$. In this region, the carrier transit time $\left(\tau_{c}\right)$ is greater than the material dielectric relaxation time $\left(\tau_{d}\right)$ and material remains in quasi-neutral state [238]. At $V=V_{t r}$, a transition from ohmic conduction to SCLC occurs as $n$ becomes larger than $n_{0}$ and $\tau_{c}$ just becomes equal to $\tau_{\mathrm{d}}$.

In region II ( $\left.V_{\mathrm{tr}}<\mathrm{V}<\mathrm{V}_{\mathrm{TFL}}\right)$ the electron quasi-Fermi level $\left(\mathrm{E}_{\mathrm{Fn}}\right)$, which is a function of injected charge in case of extrinsic type conduction, is still below the shallow trap energy level $E_{t}$. In this regime, most of the injected charge does not contribute to the current and a part of it occupies the traps. Further, the fraction $(\theta)$ of the free charge to the total charge (i.e. free and trapped charge) remains constant and doesn't vary with the applied voltage as long as $E_{F n}$ is below $E_{t}$ [228]. The conduction (in region II) follows Child's law however with a current density reduced by a factor $\theta$ (Eq.(6.2)).

Further increasing the injected free carrier density by biasing a higher voltage, moves $E_{f n}$ further up and eventually at $V=V_{T F L}, E_{F n}$ just passes over $E_{t}$. Thus, $V_{T F L}$ is defined as the threshold voltage required to fill the traps. Shortly beyond $\mathrm{V}_{\mathrm{TFL}}$, i.e. region III, a steep rise in the current ( $\alpha=6.6$ ) occurs as it rapidly recovers from its low trap-limited value to a high trap-free SCL value. Therefore region III is the transition from a trapped I - V behavior to a trap-free behavior. Finally beyond this transition region, when all traps are completely filled, they no longer affect the charge injection. Hereafter, any injected charge fully contributes to the current (region IV) and an ideal trap-free square law $(\alpha=2.06)$ is followed (Eq.(6.3)).

Using Eqs.(6.1)-(6.7) [228] and following the methodology as previously described by Chiu et al. [238], the basic electrical properties of the polyGaN material were extracted and are summarized in Table 6.1. For the calculations, $d=36 \mathrm{~nm}$ (GaN thickness) and the literature reported value of $\epsilon_{\mathrm{r}}=8.9$ for $\mathrm{GaN}$ [239] were used. $\mathrm{V}_{\mathrm{tr}}=0.31 \mathrm{~V}$ and $\mathrm{V}_{\mathrm{TFL}}=0.61 \mathrm{~V}$ were estimated from Fig. 6.6. Further, temperature dependent measurements 
Table 6.1: Extracted electrical properties of poly-GaN material at $300 \mathrm{~K}$ from the $\mathrm{J}_{\mathrm{a}}-\mathrm{V}$ analysis.

\begin{tabular}{|l|c|}
\hline Properties & Values \\
\hline $\begin{array}{l}\text { Transition voltage from ohmic to SCLC } \\
\text { regime, } \mathrm{V}_{\mathrm{tr}}\end{array}$ & $0.31 \mathrm{~V}$ \\
\hline Trap-filled-limited voltage, $\mathrm{V}_{\mathrm{TFL}}$ & $0.61 \mathrm{~V}$ \\
\hline Effective trap density, $\mathrm{N}_{\mathrm{t}}$ & $4.6 \times 10^{17} / \mathrm{cm}^{3}$ \\
\hline Trap energy level, $\mid \mathrm{E}_{\mathrm{C}}-\mathrm{E}_{\mathrm{t}} \mathrm{I}$ & $0.13 \mathrm{eV}$ \\
\hline Free carrier concentration, $\mathrm{n}_{0}$ & $4.4 \times 10^{15} / \mathrm{cm}^{3}$ \\
\hline Fermi level, I $\mathrm{E}_{\mathrm{C}}-\mathrm{E}_{\mathrm{Fn}} \mathrm{I}$ & $0.18 \mathrm{eV}$ \\
\hline Conduction band density of states, $\mathrm{N}_{\mathrm{C}}$ & $4.6 \times 10^{18} / \mathrm{cm}^{3}$ \\
\hline Ratio of free carriers to total carries, $\theta$ & 0.03 \\
\hline Electronic mobility, $\mu$ & $1.7 \times 10^{-8} \mathrm{~cm}^{2} / \mathrm{V}-\mathrm{s}$ \\
\hline Dielectric relaxation time, $\tau_{\mathrm{d}}$ & $2.4 \times 10^{-3} \mathrm{~s}$ \\
\hline
\end{tabular}

(see Fig. 6.7(a)) were used to estimate the effective $E_{t}$ from the Arrhenius plot. The energy levels $E_{F n}$ and $E_{t}$ are relative to $E_{C}$.

The Arrhenius plot of $J_{a}$ at a range of voltages in the forward bias is shown in Fig. 6.7(b) indicating a rate-limited thermally activated process $\left(J_{a} \propto \exp \left(-E_{a} / k T\right)\right)$ where $E_{a}$ is the thermal activation energy. The estimated $E_{a}$ is constant throughout the measured temperature range. This also indicates that a single trap level is dominant over the statistics of majority carriers throughout the measured temperature range [240]. Further, no significant variation $(\sim \mathrm{kT})$ in $\mathrm{E}_{\mathrm{a}}$ from ohmic conduction to SCLC regime at different voltage ranges was observed. According to Roberts and Schmidlin [240], this suggests that the conduction is largely extrinsic in nature, as also expected in wide bandgap materials. The estimated $E_{a}$ from Arrhenius plot can then be interpreted as the dominant trap energy level $E_{t}$ as long as $\theta \ll 1$. Hence, $E_{t}=0.13 \mathrm{eV} \pm 0.0259 \mathrm{eV}$ below $E_{c}$ was estimated from the extracted $E_{a}$ in the trap filling region (II) of the $\log \left(J_{a}\right)-\log (V)$ curve (Fig. 6.6). In this region, $E_{F n}$ lies below the $E_{t}$ and therefore $\theta$ which
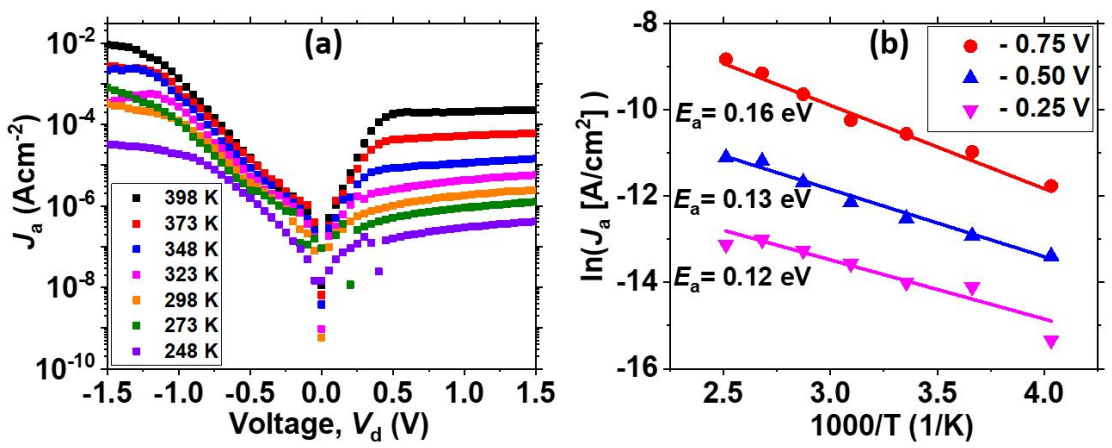

Figure 6.7: (a) Temperature dependent $J_{a}-V$ characteristics of the fabricated $\mathrm{Al} / \mathrm{GaN} / \mathrm{p}-\mathrm{Si}$ diode. (b) Arrhenius plot of $\mathrm{J}_{\mathrm{a}}$ for various forward bias levels. 

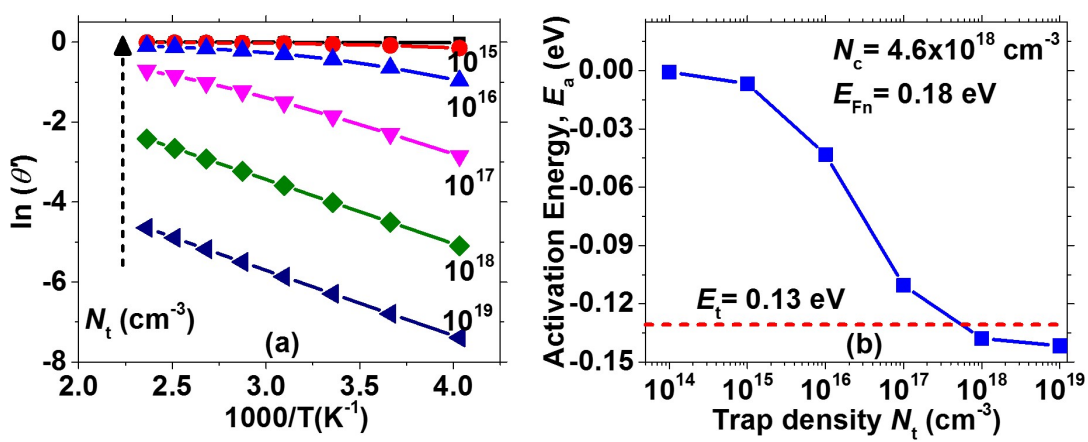

Figure 6.8: (a) Modeled Arrhenius plot of $\theta^{\prime}$ using Eq. (6.8) for various $N_{t}$. (b) Estimated $E_{a}$ against $N_{t}$ for a fixed $E_{t}=0.13 \mathrm{eV}$.

contains the $E_{a}$ for J, can be described by Eq. (6.6).

It should however be noted that Eq. (6.6) is only accurate when $\theta \ll 1$ and therefore the interpretation of $E_{a}$ from the Arrhenius plot as $E_{t}$ may be erroneous otherwise. A more generalized expression for $\theta$ as given by Eq. (6.8) [228] offers better insight into the interpretation of Arrhenius plot.

$$
\theta^{\prime}=\frac{N_{C} \cdot \exp \left[\frac{\left(E_{C}-E_{F_{n}}\right)}{k T}\right]}{N_{C} \cdot \exp \left[\frac{\left(E_{C}-E_{F_{n}}\right)}{k T}\right]+\left(\frac{N_{t}}{1+\left(1 / g_{n}\right) \cdot \exp \left[\frac{-\left(E_{t}-E_{F n}\right)}{k T}\right]}\right)},
$$

From Eq. (6.8) various cases depending on the relative positions of $E_{F n}$ and $E_{t}$ within the bandgap can be analyzed. The impact of Eq. (6.8) on our results was investigated further by inserting the estimated $E_{t}, N_{C}$ and $E_{F n}$ values from Table 6.1 there and varying the $\mathrm{N}_{\mathrm{t}}$ from $10^{14} \mathrm{till} 10^{19} / \mathrm{cm}^{3}$. The modeled Arrhenius plot along with extracted $E_{a}$ for different $N_{t}$ is shown in Fig. 6.8. It was noted that the extracted $E_{a}$ values agree with given $E_{t}$ only in a region where $N_{t} \sim N_{C}$. Since in our case, $N_{t}$ is expected to be very high because of the polycrystalline nature of the film, as also reported before [27], the measured $E_{a}$ corresponds to $E_{t}$.

Further, the role of any other possible conduction mechanism in our diodes were also investigated. It is possible that the steep slope region in the mid-voltage range $(-1<\mathrm{V}<-0.5 \mathrm{~V})$ in Fig. 6.6 could also be partly originating from the recombination tunneling mechanism [241]. This type of conduction mechanism has been reported before for wide bandgap heterojunctions $[235,236]$ in the presence of deep level traps. For a single step tunneling recombination pathway where the electrons from the conduction band of an n-type wide band gap material falls into the empty deep level trap, and subsequently tunnel into the valence band of the p-type material, the forward current density is described as $J=C_{1} N_{t} \exp (B \cdot V)$ where $C_{1}$ is a constant and $N_{t}$ is the trap density [241]. The temperature 
independent exponential pre-factor B is given as [241]:

$$
\mathrm{B}=(8 \pi / 3 \mathrm{~h})\left(\mathrm{m}_{\mathrm{h}}^{*} \epsilon_{\mathrm{r}} \epsilon_{0}\right)^{1 / 2} \mathrm{~N}_{\mathrm{D}} /\left[\mathrm{N}_{\mathrm{A}}^{1 / 2}\left(\mathrm{~N}_{\mathrm{A}}+\mathrm{N}_{\mathrm{D}}\right)\right],
$$

where $m_{h}^{*}$ is the hole effective mass, $\epsilon_{\mathrm{r}}$ is the relative permittivity of the wide band gap material, $\mathrm{N}_{\mathrm{D}}$ and $\mathrm{N}_{\mathrm{A}}$ are donor and acceptor concentration respectively and $h$ is Planck's constant. The estimated B value $(\sim 7.3$ $\mathrm{V}^{-1}$ ) from Fig. 6.7(a) via curve fitting is temperature independent in the said voltage range. However $\mathrm{J}_{\mathrm{a}}$ does show a temperature dependency in the same range unlike expected for the tunneling mechanism [241]. This suggests that recombination tunneling is not the dominant mechanism there.

Other related trap assisted tunneling (TAT) processes such as two-step TAT [242, 243] or even multi-step TAT [244] are also shown to have a weak temperature dependency. However our $\mathrm{J}_{\mathrm{a}}-\mathrm{V}-\mathrm{T}$ characteristics clearly suggest a rate-limited thermally activated process (Fig. 6.7(b)). Therefore the TAT current contribution to the forward $\mathrm{J}_{\mathrm{a}}$, though possibly present, is not significant and consequently does not affect our SCLC analysis.

The possibility of Poole-Frenkel type of conduction in our diodes [245, 246] was also considered. The $\ln \left(\mathrm{J}_{\mathrm{a}} / \mathrm{V}\right)$ vs. $\sqrt{\mathrm{V}}$ plot showed a straight line in the mid-forward voltage range as expected for Poole-Frenkel type conduction. However, the decrease in its slope with increase in temperature as also expected for Poole-Frenkel conduction was not manifest in our data. In addition, $E_{a}$ also did not decrease with the applied bias unlike in case of Poole-Frenkel conduction where the applied field reduces the effective trap ionization level. Therefore it appears that Poole-Frenkel conduction is not the dominant transport mechanism in our diodes as well.

Our $\mathrm{J}_{\mathrm{a}}-\mathrm{V}$ analysis along with its temperature dependency strongly suggest the single level shallow trap mediated SCLC as the dominant mechanism. As a sanity check on the proposed SCLC model, $\mathrm{N}_{t}$ value was also independently estimated from the intercept of the Arrhenius plot (Fig. 6.7(b)) using Eq. (6.2) and assuming $\mathrm{N}_{\mathrm{c}} \sim 1.2 \times 10^{18} / \mathrm{cm}^{3}$ for $\mathrm{GaN}$ [239]. This was found to be consistent with the previously calculated value from the $\mathrm{J}_{\mathrm{a}}-\mathrm{V}$ analysis using Eq. (6.5). Moreover, the conduction was analytically modeled using Eqs.(6.1)-(6.7) along with the extracted parameters from Table 6.1 and a good fit with the $\mathrm{J}_{\mathrm{a}}-\mathrm{V}$ plot was obtained.

\subsection{Optical Measurements}

Infrared (IR) electroluminescence (EL) was observed from the fabricated $\mathrm{Al} / \mathrm{GaN} / \mathrm{p}$-Si (Fig 6.9(a)) diode. The measured spectrum (Fig 6.9(b)) is centered around the $1.12 \mu \mathrm{m}$ wavelength corresponding to the Si bandgap $\left(E_{\mathrm{g}-\mathrm{Si}}=1.12 \mathrm{eV}\right)$ with a full-width-half-maximum $(\mathrm{FWHM}) \sim 96 \mathrm{meV}$ at $300 \mathrm{~K}$. The observed FWHM is in excess of $1.8 \mathrm{kT}$ [157] because of light being emitted from silicon, an indirect band gap semiconductor where interaction with phonons during the radiative recombination process leads 


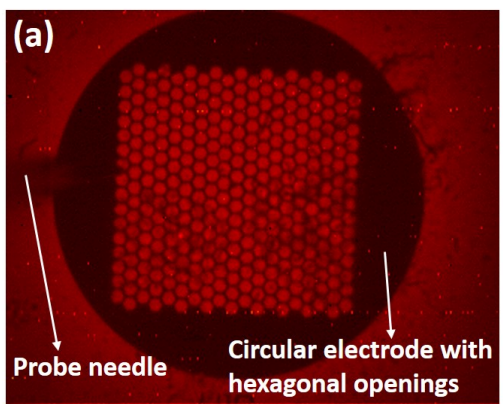

Figure 6.9: (a) Bright field IR micrograph of the fabricated GaN/p-Si diode at a constant forward current drive of $15 \mathrm{~mA}\left(\mathrm{~J}=19 \mathrm{~A} / \mathrm{cm}^{2}\right)$, (b) Optical spectrum of the emitted light from the same diode.

to the broadening of the EL-spectrum compared to that in direct band-gap semiconductors [247, 248]. Both the peak wavelength and the FWHM are in good agreement when compared with standard p-n junctions in silicon [249].

For reference sake, an Al/p-Si (with native oxide) Schottky diode [56] was also realized. From this device, faint IR emission was observed which was too weak to be detected by our spectrometer as reported before [56]. This is also in line with the detected photocurrent ( $\left.\mathrm{I}_{\mathrm{PD}}\right)$, Fig. 6.10(a), using an off-chip Si photodiode (PD) [156] which is a measure of the emission intensity corresponding to the injected diode current $\left(\mathrm{I}_{\mathrm{LED}}\right)$. For the same $\mathrm{I}_{\mathrm{LED}}$, the measured $\mathrm{I}_{\mathrm{PD}}$ and therefore emission intensity for the $\mathrm{Al} / \mathrm{GaN} / \mathrm{p}$ Si diode $\left(\mathrm{I}_{\mathrm{PD}} \sim 1 \mathrm{nA}\right)$ is $\sim 3$ orders of magnitude higher than for the $\mathrm{Al} / \mathrm{p}-\mathrm{Si}$ diode $\left(\mathrm{I}_{\mathrm{PD}} \sim 1 \mathrm{pA}\right)$. The emission intensity in the $\mathrm{GaN} / \mathrm{p}-\mathrm{Si}$ diode was also found to gradually increase with the injected power as expected from the LED behavior [157].
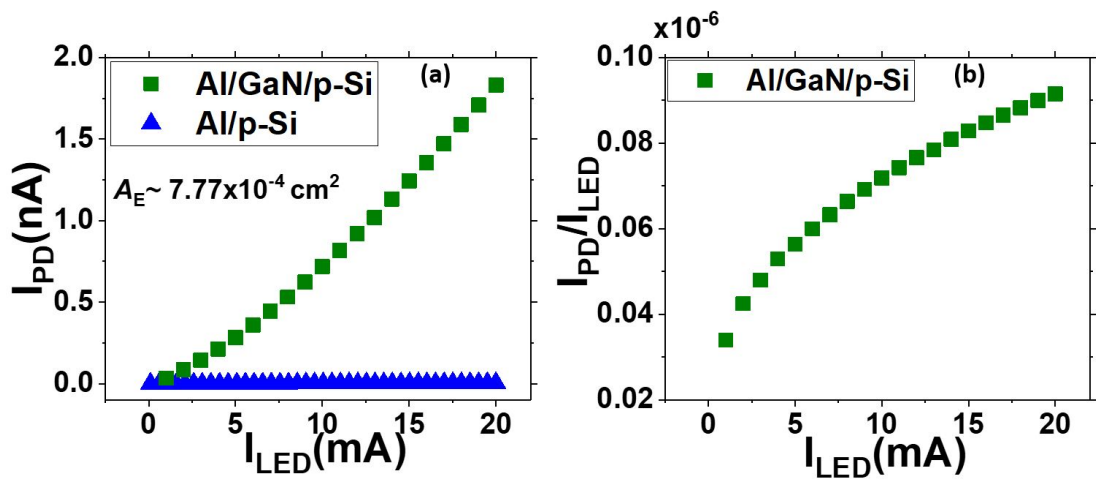

Figure 6.10: (a) Measured short-circuit current $\left(\mathrm{I}_{\mathrm{PD}}\right)$ in the photodiode (PD) versus injected forward current ( $\mathrm{I}_{\mathrm{LED}}$ ) for the $\mathrm{Al} / \mathrm{GaN} / \mathrm{p}$-Si diode and $\mathrm{Al} / \mathrm{p}$-Si diode [56]. (b) $\mathrm{I}_{\mathrm{PD}} / \mathrm{I}_{\mathrm{LED}}\left(\propto \eta_{\mathrm{LED}}\right)$ versus $\mathrm{I}_{\mathrm{LED}}$. 
Further, the $\mathrm{I}_{\mathrm{PD}} / \mathrm{I}_{\mathrm{LED}}$ ratio, is proportional to the internal quantum efficiency (IQE) of the LED. This is so because $\mathrm{I}_{\mathrm{PD}} / \mathrm{I}_{\mathrm{LED}}=\eta_{\mathrm{PD}} \cdot \eta_{\text {ext. }} \cdot \eta_{\mathrm{LED}}$, where $\eta_{\mathrm{PD} / \text { ext./LED }}$ is the IQE of detection in the PD/extraction efficiency of light/IQE of the LED, respectively [249]. Out of the three efficiencies, only $\eta_{\text {LED }}$ is a function of $I_{L E D}$. Thus, the trend in $\eta_{\text {LED }}$ versus $I_{L E D}$ is reflected in the trend in $\mathrm{I}_{\mathrm{PD}} / \mathrm{I}_{\mathrm{LED}}$ versus $\mathrm{I}_{\mathrm{LED}}$. As shown in the Fig. 6.10(b), in the low injection regime, $\eta_{\text {LED }}$ was found to be increasing for higher $\mathrm{I}_{\mathrm{LED}}$ and no droop was observed up till an injected current density $\sim 25 \mathrm{~A} / \mathrm{cm}^{2}$.

Based on our measured $I_{P D}$ (with a $\eta_{\mathrm{PD}}$ of 0.2 at $\sim 1120 \mathrm{~nm}$ free-space wavelength [156]), the external quantum efficiency of our LED (at $\mathrm{I}_{\mathrm{LED}}=10$ $\mathrm{mA}$ ) was estimated to be $3.5 \times 10^{-7}$. Further, from an estimate of the optical extraction efficiency $\left(\sim 1.5 \times 10^{-3}\right)$ of our measurement set-up [250], the lower limit of the $\eta_{\text {LED }}$ of our LED was calculated to be $\sim 2 \times 10^{-4}$ (at $\mathrm{I}_{\mathrm{LED}}=10 \mathrm{~mA}$ ) without taking into account losses at the top electrode which blocks majority of emission, allowing only light from the periphery of the hexagonal openings (Fig 6.9(a)) to be detected.

The observed infrared EL from the GaN/p-Si device is attributed to the recombination of the injected minority carriers (electrons) with the background majority carriers (holes) at the silicon side of the GaN/p-Si interface as illustrated in Fig. 6.3. Therefore the electron current component determines the light emission. Further, no ultra-violet (UV) emission originating from the GaN band-edge was observed due to high valence band offset at GaN/p-Si interface which indicates negligible hole concentration in the GaN layer. The role of the single type of charge carrier (i.e., electrons) in the conduction in GaN layer is also confirmed by the observed SCLC behavior. Higher IR emission in case of GaN/p-Si diode can be attributed to the wide band gap of the (n-type) GaN layer and resultant band discontinuity at the GaN/p-Si interface which improves its (minority) injection efficiency compared to Al/p-Si diode. Previously, enhanced emission from $\mathrm{Si}$ in the presence of native oxide has been reported [251]. However, in our case, no conclusive evidence of any possible role of the native oxide in the observed EL could be established. Both our diodes i.e. $\mathrm{Al} / \mathrm{GaN} / \mathrm{p}-\mathrm{Si}$ and $\mathrm{Al} / \mathrm{p}$-Si had a native oxide.

Fig. 6.11 which shows measured I $-\mathrm{V}$ characteristics of two different devices; the $\mathrm{Al} / \mathrm{p}-\mathrm{Si}$ and $\mathrm{Al} / \mathrm{GaN} / \mathrm{p}-\mathrm{Si}$ diode, sheds more light on the observed EL characteristics. The data indicate that for the same injected current level $\mathrm{I}_{\mathrm{LED}}$, as subjected to our EL measurements, the internal junction voltage $V_{j}$ increases for devices with a reduced current level. Moreover, for the GaN/p-Si diode the electron current is its actual current depicted in Fig. 6.11. Since it is limited by the GaN layer that component will be less than in the $\mathrm{Al} / \mathrm{p}$-Si diode. As a result, $\mathrm{V}_{\mathrm{j}-\mathrm{pSi}}<\mathrm{V}_{\mathrm{j}-\mathrm{pSiGaN}}$. Consequently, the product of the hole and electron density (i.e., $p \cdot n$ ) in Si (light emitting region in both the cases) which is exponentially dependent on $V_{j}$, also follows the same order $\left(p \cdot n_{\mathrm{pSi}}<\mathrm{p} \cdot n_{\mathrm{pSiGaN}}\right)$. This explains the enhanced emission from the GaN/Si diode compared to that of the Si-only diode, 


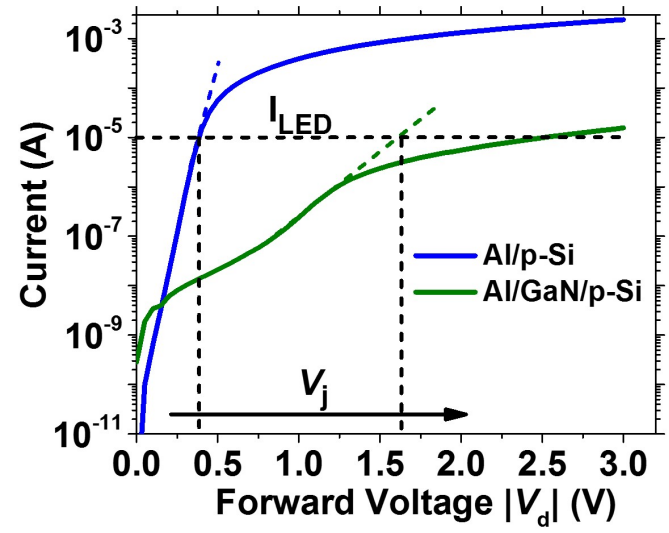

Figure 6.11: Measured I-V characteristics of $\mathrm{Al} / \mathrm{GaN} / \mathrm{p}-\mathrm{Si}$ diode and $\mathrm{Al} / \mathrm{p}-\mathrm{Si}$ diodes.

since a higher $p \cdot n$-value yields a higher radiative recombination rate (i.e., light emission) [158].

\subsection{Discussion}

From the analysis discussed before, it can be concluded that the current flow in our ALD-grown thin-film poly-GaN/Si diode is largely limited by the GaN layer ${ }^{3}$. This allowed us to estimate the basic electrical properties of the polycrystalline layer via $\mathrm{I}-\mathrm{V}$ analysis using the single shallow trap SCLC model [228]. The relatively large trap density in the unintentionally doped thin film of the wide band gap material could be the reason for the observed SCLC behavior. However, in a good heterojunction [252], for electronic applications such as bipolar transistors, the current should be limited by diffusion in the narrow band gap material.

Despite this, the optical measurements indicate that such engineered poly-GaN based heterojunctions, realized at low temperature, could be interesting for fabricating efficient $S i$ based IR emitters using a relatively simple process. Such heterojunctions can also be investigated for Si based solar cells with an improved injector efficiency [234, 253, 254]. Heterojunction diodes utilizing wide bandgap materials as an emitter are interesting for enhancing the injector efficiency, for example, of the electroluminescent diode [1] and have been reported before for different materials [251, 255259]. For instance, Favennec et al. [255] reported the improved luminescence from Er when implanted in a wide band gap materials matrix. More recently, near IR emission peaking at $826 \mathrm{~nm}$ from GaN/Si heterojunctions was also reported by Han et al. [259, 260], where unlike in our process,

\footnotetext{
${ }^{3}$ More detailed discussion on electrical properties of ALD-grown poly-GaN thin films will be followed in chapter 7 .
} 
a $500 \mathrm{~nm}$ thick $\mathrm{GaN}$ nanocrystalline film was grown at $1050{ }^{\circ} \mathrm{C}$ with a CVD process on an Si nanoporous pillar array (NPA). The difference in the observed peak EL wavelength with that obtained from our $\mathrm{GaN} / \mathrm{Si}$ devices is due to the reported higher energy bandgap $\left(\mathrm{E}_{\mathrm{g}-\mathrm{Si}-\mathrm{NPA}}=2 \mathrm{eV}\right)$ and electron affinity $\left(\chi_{\mathrm{Si}-\mathrm{NPA}}=3.6 \mathrm{eV}\right)$ of the Si NPA and therefore resulting in different band alignment and heterojunction properties. Interestingly, the conduction mechanism reported for the GaN/Si-NPA device [260] was also attributed to an SCLC process.

\subsection{Conclusions}

It was shown that heterojunction diodes can be realized at a low temperature by adopting ALD of poly-GaN on an Si wafer. The fabricated heterojunction devices showed good rectification and also infrared EL. The current transport in the forward bias is explained by SCLC in the presence of traps as possible dominant transport mechanism. Following the single trap SCLC model along with temperature dependent $J-V$ analysis, a minimum trap density of $4.6 \times 10^{17} / \mathrm{cm}^{3}$ at an energy level $0.13 \mathrm{eV}$ below the conduction band minimum was estimated in the polycrystalline film. Other electrical properties of the poly-GaN layer such as free carrier concentration, density of states in the conduction band, electronic mobility and dielectric relaxation time were also determined using the SCLC analysis. The observed IR emission from EL is attributed to band-to-band recombination in the Si near the heterojunction interface because of a high $p \cdot n$-value. The presence of a thin polycrystalline GaN significantly improves the IR emission. The introduction of such engineered heterostructures could be a way forward for making efficient optoelectronic devices such as IR emitters and solar cells realized in bulk Si. The study of such thin-film ALD GaN on $\mathrm{Si}$ is also promising for future nanoscale (opto)electronics based on this platform. 



\title{
ELECTRICAL PROPERTIES OF ALD-GROWN POLY-GAN THIN-FILMS
}

\begin{abstract}
This chapter deals with the basic electrical conduction properties of polycrystalline gallium nitride (poly-GaN) thin films. These films were fabricated by means of two different atomic-layer deposition (ALD) processes namely plasma-enhanced ALD and thermal ALD. Their electrical properties were systemically investigated and compared using electrical measurements on simple device test structures. Based on this study, potential applications of such materials are proposed and discussed.
\end{abstract}




\subsection{Introduction}

Polycrystalline (poly-) GaN is an interesting material for various thin-film device applications and also from $\mathrm{GaN}$-on-Si integration perspective as outlined previously in chapter 1 as well as in chapter 6 . It is expected that the electrical properties of poly-GaN thin-films are different from their crystalline counterparts. Therefore it is imperative to investigate the electrical properties of these films in order to predict their possible applications in semiconductor technology.

In this work, two different types of atomic-layer deposition (ALD) techniques, as developed by S. Banerjee [223] in the joint research project, were utilized to grow poly-GaN thin films. The first technique, referred to as plasma-enhanced ALD (PEALD), utilizes a plasma source to dissociate $\mathrm{NH}_{3}$ for creating reactive nitrogen radicals during an ALD cycle for the $\mathrm{GaN}$ formation. The use of a plasma source enhances the reaction rate by creation of radicals and/or ions and allows it to proceed at a relatively low temperature which otherwise would have been energetically unfavorable. The PEALD process has been utilized to realize $\mathrm{GaN} / \mathrm{p}$-Si heterjunction diodes as described in chapter 6 .

The second technique, referred to as thermal ALD, relies on pure thermal-mode for GaN formation via the surface adduct mechanism as described in more detail in [223, 261]. The $\mathrm{NH}_{3}$ precursor is then not dissociated by striking a plasma, instead the reaction proceeds by forming a so-called TMG: $\mathrm{NH}_{3}$ surface adduct. These surface adducts subsequently link together and convert into $\mathrm{GaN}$ units by releasing $\mathrm{CH}_{4}$.

In both techniques, the $\mathrm{GaN}$ film is grown at $400^{\circ} \mathrm{C}$ using trimethylgallium (TMG) and $\mathrm{NH}_{3}$ as precursors. From hereafter, the poly-GaN thin-films grown by PEALD and thermal ALD will be abbreviated as P$\mathrm{GaN}$ and T-GaN respectively. The material characterization results of P-GaN film has been briefly discussed in chapter 6 . The layer was found to be $\mathrm{N}$-rich with negligible carbon and about $7 \%$ oxygen inside the film. In comparison, $\mathrm{T}-\mathrm{GaN}$ film is $\mathrm{Ga}$ rich with an average composition estimated as $\mathrm{Ga}_{0.53} \mathrm{~N}_{0.39} \mathrm{C}_{0.01} \mathrm{O}_{0.07}$ [223]. Both films confirm to polycrystalline wurzitic structure. Table 7.1 summarizes the key features of the P-GaN and T-GaN growth techniques and their respective film qualities. Next, the basic electrical properties of both P-GaN and T-GaN are investigated and compared using simple device test structures. To obtain better insight into the conduction properties of the poly-GaN material in vertical as well as lateral direction, both T-GaN and P-GaN layers were grown on $\mathrm{Si}, \mathrm{SiO}_{2}$ and sapphire substrates.

The polycrystalline GaN thin-film ALD growth and device fabrication as reported in this chapter were mainly performed by S. Banerjee as part of the joint research project. 
Table 7.1: Comparison of key features of P-GaN and T-GaN ALD growth techniques [223].

\begin{tabular}{|l|l|l|}
\hline Key features & P-GaN & T-GaN \\
\hline Tool & $\begin{array}{l}\text { Picosun R-200 } \\
\text { remote plasma }\end{array}$ & Cluster ALD \\
\hline Precursors & TMG + $\mathrm{NH}_{3}$ & TMG + $\mathrm{NH}_{3}$ \\
\hline Growth temperature & $400^{\circ} \mathrm{C}$ & $400^{\circ} \mathrm{C}$ \\
\hline Process & $\begin{array}{l}\mathrm{NH}_{3} \text {-plasma ac- } \\
\text { tivated }\end{array}$ & $\begin{array}{l}\text { surface adduct } \\
\text { mechanism }\end{array}$ \\
\hline Composition & N-rich & Ga-rich \\
\hline H-content & low & high \\
\hline Crystallinity & polycrystalline & polycrystalline \\
\hline $\begin{array}{l}\text { Crystal structure (dom- } \\
\text { inant) }\end{array}$ & wurtzite & wurtzite \\
\hline
\end{tabular}

\subsection{Experimental details}

The P-GaN films were deposited using a commercial remote plasma R-200 Picosun reactor, while T-GaN was grown in a home-made ALD cluster system. Both films were deposited at $\sim 400^{\circ} \mathrm{C}$ using $\mathrm{TMG}$ and $\mathrm{NH}_{3}$ as precursors. In addition, the AlN film (wherever mentioned) was deposited using the same reactor without breaking the vacuum. Poly-GaN films deposited on sapphire substrates were utilized for lateral electrical measurements. For back-gated TFT realization, highly doped $\left(\sim 10^{18} \mathrm{~cm}^{-3}\right)$ p-type Si substrates (4-inch) with $\sim 50 \mathrm{~nm}$ thermally grown silicon dioxide $\left(\mathrm{SiO}_{2}\right)$ layer on top were used. For investigating the vertical current component, p-type Si(111) substrates (with native oxide) with a background doping concentration of $10^{15} \mathrm{~cm}^{-3}$ (as reported in chapter 6) were utilized.

Before the film deposition, sapphire substrates were cleaned in acetone, while $\mathrm{SiO}_{2} / \mathrm{Si}$ substrates were cleaned using $99 \%$ and $69 \%$-by-vol. $\mathrm{HNO}_{3}$ sequentially to remove organic and metallic contaminants respectively. For electrodes, Al metal (low work function) electrodes were realized on the top poly-GaN surface using a standard photolithography and wet etch process. For realizing Pd (high work function) electrodes, a lift-off process was utilized instead of wet etching. The substrate bottom was used as a back contact for vertical measurement. Surface passivation (wherever mentioned) was done using a $165 \mathrm{~nm}$ PECVD grown silicon nitride $\left(\mathrm{Si}_{3} \mathrm{~N}_{4}\right)$. Finally, unless mentioned otherwise, no heat treatment was given to any of the prepared samples after contact metalization. 


\subsection{Electrical properties of P-GaN}

\subsubsection{Vertical conduction in P-GaN}

Vertical conduction is possible in P-GaN thin-films, as evident from the results discussed in the previous chapter where a rectifying behavior was observed in the P-GaN/p-Si heterojunction diode. The charge transport was found to be largely limited by the space charge limited current (SCLC) mechanism in the P-GaN layer. Both the areal $\left(\mathrm{J}_{\mathrm{a}}\right)$ and peripheral $\left(\mathrm{J}_{\mathrm{p}}\right)$ current component in the vertical diode were studied. The root cause of the significant $\mathrm{J}_{\mathrm{p}}$ could not be established before. The high $\mathrm{J}_{\mathrm{p}}$ could be due to the presence of a surface conductive channel in the P-GaN film as analyzed further in this chapter.

\subsubsection{Lateral conduction in P-GaN}

\section{Hall measurements}

Hall measurements [262] were performed ${ }^{1}$ in order to estimate the charge carrier density and their polarity i.e. n-type or p-type, in the P-GaN film. The P-GaN/sapphire sample with a film thickness of $40 \mathrm{~nm}$ was used for this purpose. The sample was first diced into $8 \mathrm{~mm} \times 8 \mathrm{~mm}$ pieces using a laser cutting tool and then point contacts were formed at four edges of the square samples by depositing $\mathrm{Al}$ metal via a shadow mask as shown schematically in Fig. 7.1(a). The Hall measurements were performed for a varying magnetic field strength, $\mathrm{B}$, from $-9 \mathrm{~T}$ till $9 \mathrm{~T}$ at various temperatures $(100 \mathrm{~K}-300 \mathrm{~K})$. The excitation current, I, was in the range of $0.8 \mu \mathrm{A}$. The relation between the Hall voltage, $V_{\mathrm{H}}$, and sheet carrier density, $\mathrm{n}_{\mathrm{sh}}$ of the

\footnotetext{
${ }^{1}$ The measurements were performed using Quantum Design Physical Properties Measurement System (PPMS) in temperature-controlled environment and under dark conditions.
}
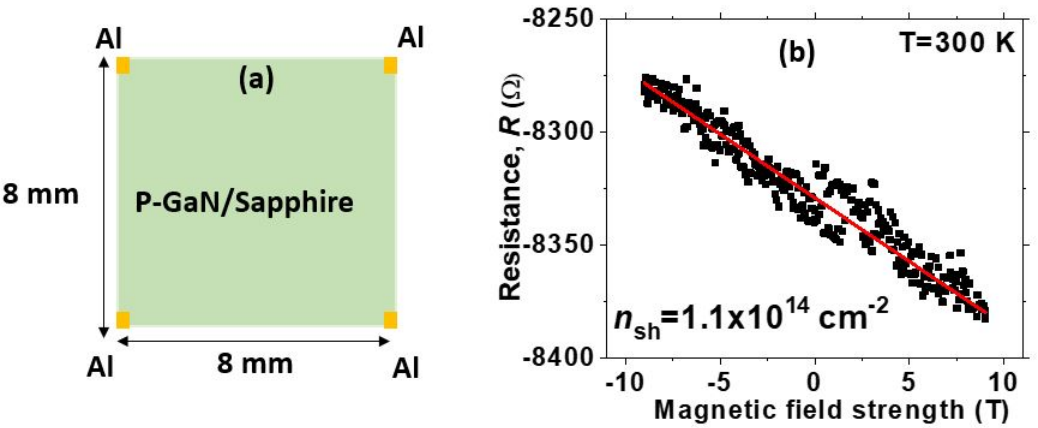

Figure 7.1: (a) Schematic top view of P-GaN (40 nm)/sapphire sample used for Hall measurements and (b) Measured resistance, $R$, for a varying magnetic field strength at $300 \mathrm{~K}$. 
film can be described as [262]:

$$
\frac{V_{H}}{I B}=\frac{R}{B}=\frac{-r}{n_{s h} q},
$$

The $r$ is the scattering factor and is assumed to be unity in our measurements [262]. Here, we consider this relation in terms of effective measured resistance, $R\left(=V_{H} / I\right)$, instead of Hall voltage, $V_{H}$, as the former is independent of the measurement current. The sheet carrier density, $n_{\mathrm{sh}}$ and carrier type can then be determined (using appropriate units) from the slope of measured resistance ( $R$ ) against the magnetic field (B) using Eq. (7.1). Other than reducing the uncertainty of relying over two data points $( \pm B)$ in conventional Hall analysis, the slope method [263] also eliminates the unwanted contribution of any constant offset voltage which appears across the diagonally opposite terminals at $\mathrm{B}=0 \mathrm{~T}$ independent of the measurement current. The finite offset voltage may originate from thermoelectric effects or asymmetry of the contact electrodes or measurement sample itself. In addition to Hall measurements, the van-der-Pauw measurements were performed at zero magnetic field for the sheet resistance $\left(\mathrm{R}_{\mathrm{sh}}\right)$ calculation.

The negative slope of the R vs B plot in Fig. 7.1(b) suggests that electrons are involved in the conduction process. The sheet carrier density, $n_{\text {sh }}$, as extracted from the slope of this curve is $\sim 10^{14} \mathrm{~cm}^{-2}$ at $300 \mathrm{~K}$. Assuming that the carriers are uniformly distributed across the film layer thickness then for the $40 \mathrm{~nm}$ film it would be equivalent to a (volume) carrier concentration of $\sim 2.5 \times 10^{19} \mathrm{~cm}^{-3}$. The sheet resistance, $R_{s h}$, obtained from resistivity measurements, is $\sim 433 \mathrm{k} \Omega / \square$ at $300 \mathrm{~K}$. For the $40 \mathrm{~nm}$ film it is equivalent to a "bulk" resistivity $\rho \sim 1.7 \Omega-\mathrm{cm}$. Using the $n_{\mathrm{sh}}$ and $\mathrm{R}_{\mathrm{sh}}$ values, the carrier Hall mobility, $\mu_{\mathrm{n}}$ is calculated to be $\sim 0.16 \mathrm{~cm}^{2} / \mathrm{V}-\mathrm{s}$ at $300 \mathrm{~K}$. The observed noise (scattered data) in the measured data can be attributed to the relatively high resistance (low carrier mobility) of the experimental sample which limits the excitation current to be below $1 \mu \mathrm{A}$.

Electrical measurements were performed for several temperatures to analyze the conduction properties as shown in Fig. 7.2. The sheet resistance (conductance) decreases (increases) with the temperature. The positive temperature coefficient of the conductance also suggests a semiconductorlike behavior of the channel and rules out the possibility of a metal like film (e.g. metallic Ga). This is confirmed by the fact that no signature of elemental Ga i.e., Ga-Ga bonds was observed in the X-ray photoelectron spectroscopy (XPS) analysis of P-GaN film as reported by S. Banerjee [223]. The sheet carrier density, $n_{\text {sh }}$, shows a decreasing trend with temperature. For a semiconductor, $n_{\text {sh }}$ should either increase or remain constant with the temperature. Therefore, considering the relatively large error $\left(\sim 7 \times 10^{13}\right.$ $\mathrm{cm}^{-2}$ ) in the $n_{\mathrm{sh}}$ extraction for lower temperatures, the small variation in measured $n_{\text {sh }}$ with temperature are ignored and it is treated as a constant $\left(\sim 10^{14} \mathrm{~cm}^{-2}\right)$ for our further analysis. However, Hall measurements should be repeated at more temperatures for confirmation. The mobility, $\mu_{n}$, on 

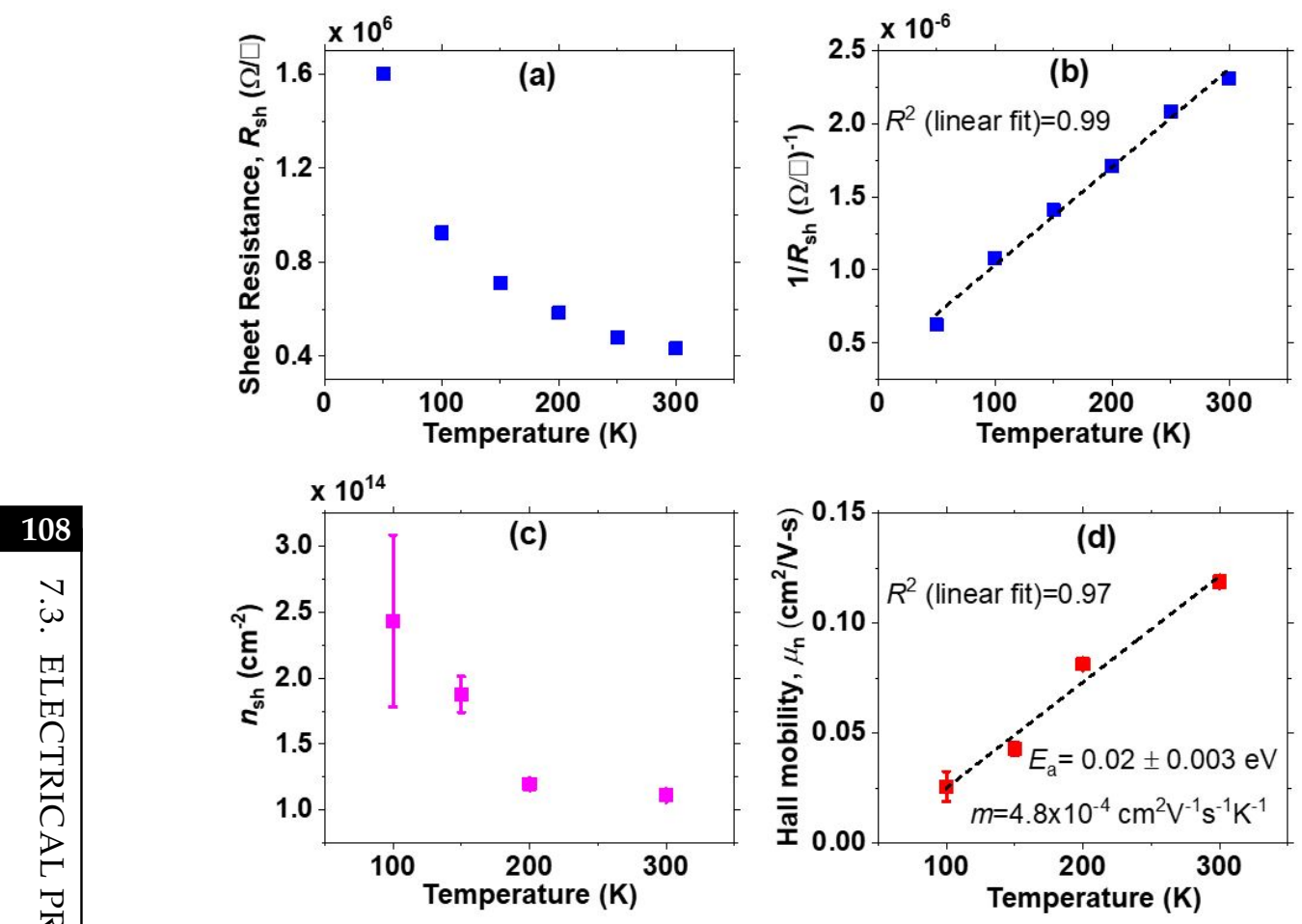

Figure 7.2: P-GaN layer properties extracted from Hall measurements at various temperatures, (a) sheet resistance, $R_{\mathrm{sh}}$, (b) sheet conductance, $\mathrm{R}_{\mathrm{sh}}^{-1}$, (c) sheet carrier density, $n_{\text {sh }}$ and (d) Hall mobility, $\mu_{n}$. The thermal activation energy, $E_{a}$, of $\mu_{n}$ estimated from its Arrhenius diagram i.e. $\ln \left(\mu_{n}\right)-\mathrm{T}^{-1}$ plot (not shown) is $\sim 0.02$ $\mathrm{eV}$.

the other hand, clearly increases almost linearly with the temperature. The trend of mobility and inverse of sheet resistance (measure of conductance) against the temperature are further reflected in the $\mathrm{I}-\mathrm{T}$ characteristics, as discussed in the next section (ref. Fig. 7.6(b)).

The extracted layer properties (at room temperature) of the P-GaN film grown on a sapphire substrate are also in good agreement with our less extensive measurements on various other $\mathrm{P}-\mathrm{GaN}$ samples both on sapphire and $\mathrm{SiO}_{2} / \mathrm{Si}$ substrates.

It is however important to note that such a standard dc Hall measurement procedure is prone to possible errors due to the polycrystalline nature of the film with a low mobility $\left(<1 \mathrm{~cm}^{2} / \mathrm{V}-\mathrm{s}\right)$ and therefore should be taken with some caution [263]. For a higher accuracy, the procedures as outlined by Werner [263] should be followed. Interestingly, a reasonably good fits to measured I - T (ref. Fig. 7.6 (b)) and I - V (ref. Fig. 7.11(b)) characteristics were obtained using the $n_{\mathrm{sh}}$ and $\mu_{\mathrm{n}}$ information obtained from Hall measurements. 


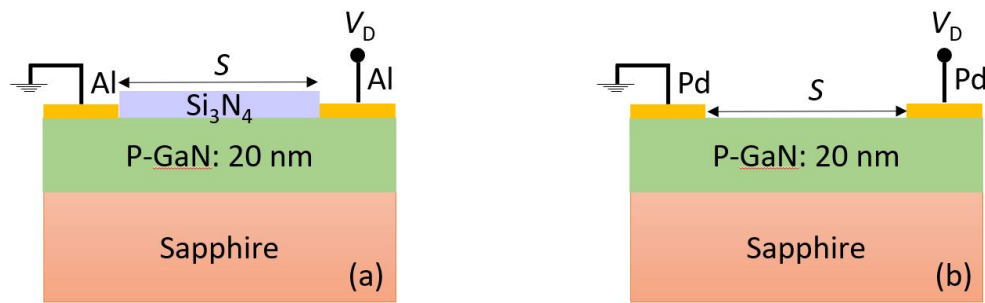

Figure 7.3: Schematic cross-sections of experimental P-GaN/sapphire devices, (a) with $\mathrm{Al}$ contacts and $\mathrm{Si}_{3} \mathrm{~N}_{4}$ surface passivation, (b) with Pd contacts and no surface passivation.

$\mathrm{I}-\mathrm{V}-(\mathrm{T})$ characteristics

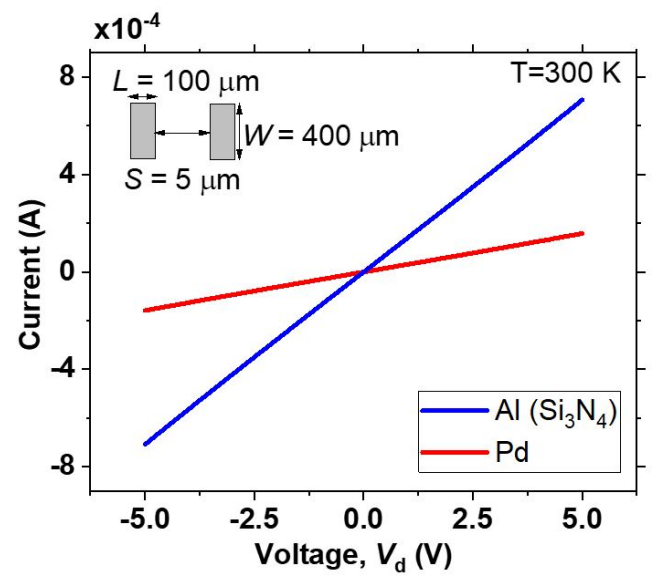

Figure 7.4: $\mathrm{I}-\mathrm{V}$ characteristics of $\mathrm{P}-\mathrm{GaN} /$ sapphire devices with $\mathrm{Al}\left(\mathrm{Si}_{3} \mathrm{~N}_{4}\right.$ passivation) and Pd contacts. Device width $W=400 \mu \mathrm{m}, \mathrm{T}=300 \mathrm{~K})$.

Fig. 7.3 shows the schematic cross-sections of the experimentally realized devices for studying lateral conduction properties of $\mathrm{P}-\mathrm{GaN}$ thin films. The P-GaN thin-film grown on sapphire substrate showed linear I - V characteristics in the lateral direction irrespective of using metal electrodes with a high $(\mathrm{Pd})$ and low $(\mathrm{Al})$ work function, as shown in Fig. 7.4. Interestingly, even a point-contact using a (tungsten) probe tip directly on the P-GaN film showed a linear behavior. The observed linearity in the I $-\mathrm{V}$ characteristics sustained even after passivating the P-GaN surface with an $\mathrm{Si}_{3} \mathrm{~N}_{4}$ layer ( $165 \mathrm{~nm}$ ) suggesting that the conduction is determined by intrinsic properties of the P-GaN film. Similar linear I - V characteristics were also observed for various other P-GaN samples grown on sapphire as well as $\mathrm{SiO}_{2} / \mathrm{Si}$ substrates with good reproducibility.

The observed conduction behavior irrespective of the metal electrode work function, surface passivation condition and substrate type, suggests the possibility of a high carrier density near the electrode which facilitates tunneling at the metal-semiconductor (MS) interface. This is confirmed 

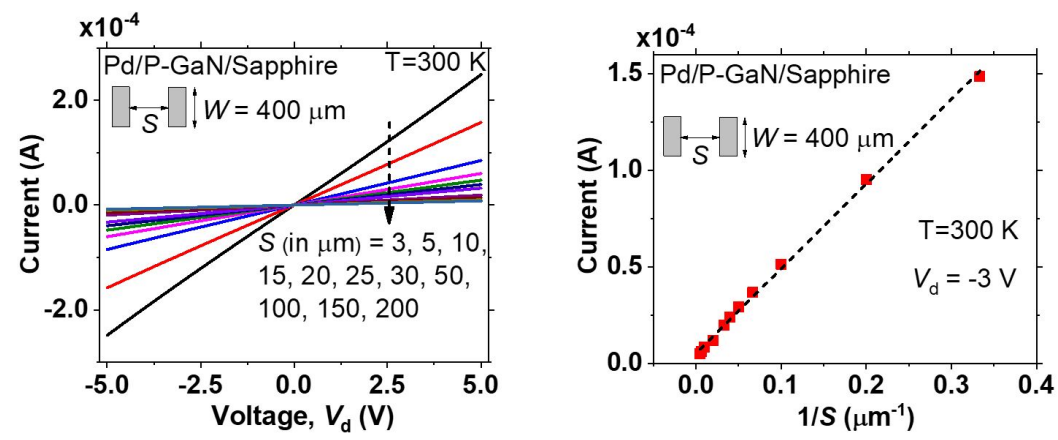

Figure 7.5: (a) I - V characteristics of $\mathrm{Pd} / \mathrm{P}-\mathrm{GaN} /$ sapphire devices for varying spacing, $S$, between the contacts. (b) Current vs inverse of contact spacing $(1 / S)$ at a fixed voltage $V_{d}=-3 \mathrm{~V}(\mathrm{~W}=400 \mu \mathrm{m}, \mathrm{T}=300 \mathrm{~K})$.

with the Hall measurements where a high sheet electron density, $n_{\mathrm{sh}} \sim 10^{14}$ $\mathrm{cm}^{-2}$, was obtained before. The channel conductivity remains unaffected even in the presence of UV light $(\lambda=255 \mathrm{~nm})$ which once again indicates the presence of a high electron density in the grown film. Moreover this type of conduction behavior was observed for various electrode spacings (2-200 $\mu \mathrm{m})$ as shown in Fig. 7.5(a), which indicates that the observed conduction behavior is not a local effect. Further, the current scales linearly with inverse of the distance between the electrodes (see Fig. 7.5(b)) which also suggest the pure resistive (ohmic) nature of the conduction channel and can simply be described as:

$$
\mathrm{I}=\mathrm{qW} \mathrm{n}_{\mathrm{sh}} \mu_{\mathrm{n}} \frac{\mathrm{V}_{\mathrm{d}}}{\mathrm{S}}
$$

where $V_{d}$ is the applied bias, $W$ is the width of the contact and $S$ is the contact spacing.

Temperature dependent I - V measurements of the P-GaN/sapphire
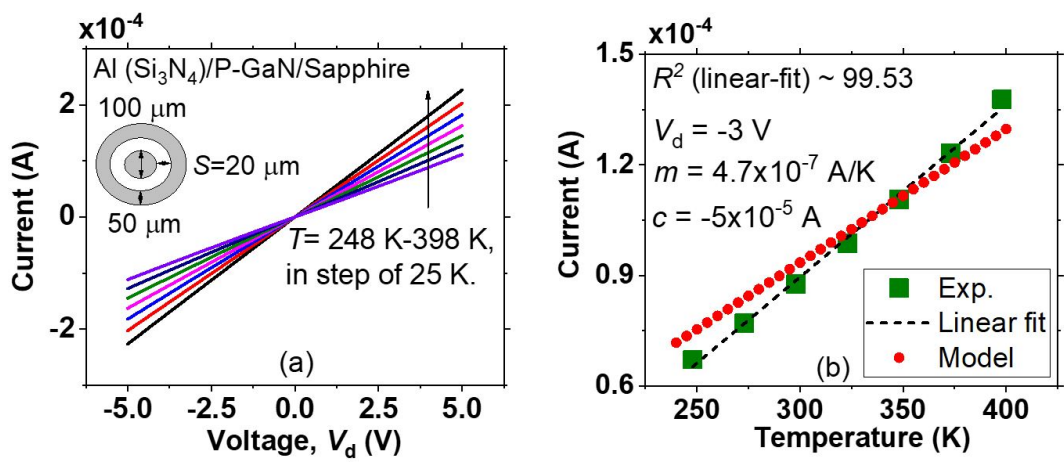

Figure 7.6: (a) Temperature dependent $\mathrm{I}-\mathrm{V}$ characteristics of $\mathrm{Al} / \mathrm{P}-\mathrm{GaN} /$ sapphire device, (b) $\mathrm{I}-\mathrm{T}$ plot at a fixed voltage $V_{d}=-3 \mathrm{~V}$. Here Eq. 7.2 is used to model the measured $\mathrm{I}-\mathrm{T}$ curve using temperature dependent mobility and carrier sheet density data obtained from the Hall analysis. 
sample were also performed, as shown in Fig. 7.6(a), where a similar linear $\mathrm{I}-\mathrm{V}$ behavior was observed for $\mathrm{T}=248 \mathrm{~K}-398 \mathrm{~K}$. The current scales linearly with the temperature as shown in Fig. 7.6(b). The observed positive temperature coefficient of the conductance reveals the semiconductor nature of the channel and rules out the possibility of a metal like film as also concluded previously from the Hall analysis. Interestingly, the observed I-T variation in Fig. 7.6(b) correlates well (both qualitatively and quantitatively) when modeled with Eq. 7.2 and using $\mu_{n}, n_{s h}$ and their temperature dependencies as obtained from the Hall measurements (refer to Fig. 7.2(c)(d)). This implies that the observed (linear) temperature dependency of the current is mainly caused by the change in the carrier mobility with the temperature. In order words, both current (conductivity) and mobility have an identical temperature dependence. Here, the $n_{\mathrm{sh}}\left(\sim 10^{14} \mathrm{~cm}^{-2}\right)$ is considered to be largely temperature independent as discussed before in respect to Fig. 7.2(c).

Note that the observed temperature dependency of $\mu_{n}$ can not be attributed to the thermal annealing effect where the film quality has possibly improved during temperature dependent measurements. The $\mu_{\mathrm{n}}$ is found to vary uniformly with temperature even down to $100 \mathrm{~K}$ where no such annealing is expected to occur.

In order to get further deeper insight into the temperature-dependent conduction property of the film, it is important to discern the dominant charge transport mechanism. Random surface potential fluctuations in the polycrystalline film could lead to carrier localization as illustrated in Fig. 7.7 [264]. Such carrier localization could result in 2-D transport properties as reported before for inversion layers in an Si-MOSFET [265]

metallic conductivity

(a)

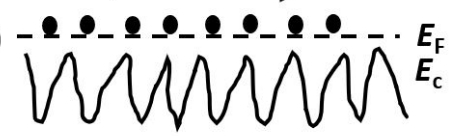

Thermally activated transport $\sigma=\sigma_{\min } \exp \left(-\left(E_{\mathrm{c}}-E_{\mathrm{F}}\right) / k T\right)$

(b)

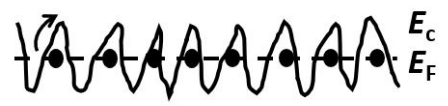

(d)

thermal activation

Variable range hopping transport

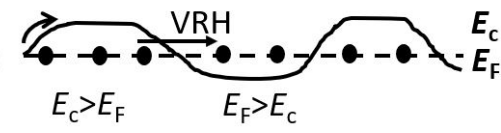

Insulating Metallic $\sigma=\sigma_{0} \exp \left(-\left(T_{0} / T\right)^{1 / 3}\right)$

(c)

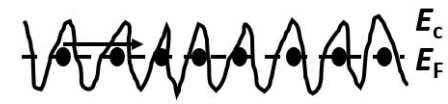

Figure 7.7: Graphical representation of random surface potential fluctuations in the conduction band of the polycrystalline material. (a)-(c) short-range fluctuations and (d) long-range fluctuations (after [264]). 

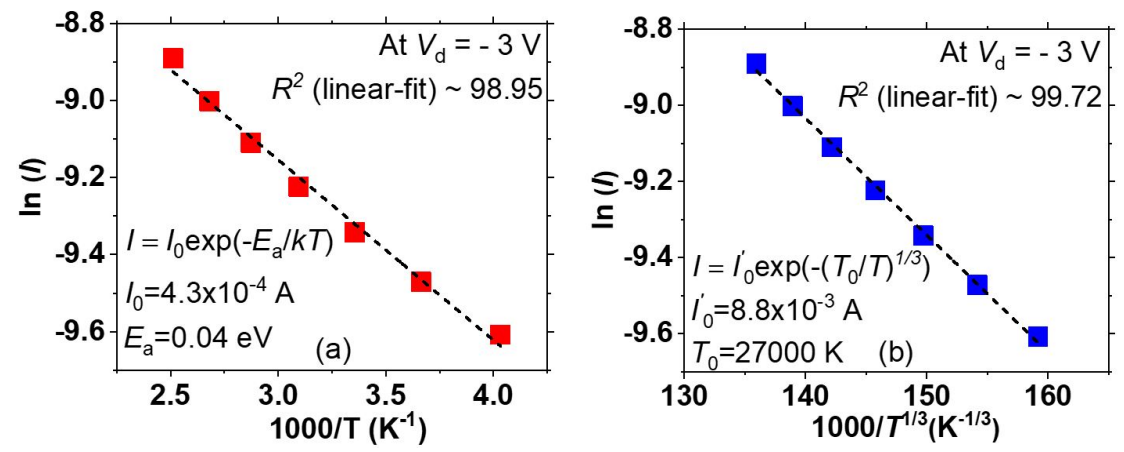

Figure 7.8: (a) $\ln (\mathrm{I})-\mathrm{T}^{-1}$ and (b) $\ln (\mathrm{I})-\mathrm{T}^{-1 / 3}$ at a fixed voltage $\mathrm{V}_{\mathrm{d}}=-3 \mathrm{~V}$ obtained from the measured $\mathrm{I}-\mathrm{T}$ dependence in Fig. 7.6(b).

and other material systems, such as hydrogen-terminated diamond [266], where transport takes place via a surface channel.

According to the ideal Anderson mobility-edge model [267], thermally activated transport takes place as a result of disorder-induced localization of carriers. For a high electron density, such that $E_{F}>E_{C}$, metallic conduction in extended states is predicted as shown in Fig. 7.7(a). While, for the case where $E_{C}>E_{F}$, at a sufficiently high temperature, charge transport proceeds via thermal excitation of electrons from a localized state to an extended state as shown in Fig. 7.7(b). In this situation, the current (hence conductivity), $I \propto \exp \left(-E_{a} / k T\right)$, where $E_{a}=\left(E_{C}-E_{F}\right)$ is the activation energy and $k$ is Boltzmann's constant. At very low temperatures, thermallyactivated tunneling between the localized states, i.e. variable range hopping $(\mathrm{VRH})$ is dominant, where $\mathrm{I} \propto \exp \left(-\left(\mathrm{T}_{0} / \mathrm{T}\right)^{1 / 3}\right)$ as illustrated in Fig. 7.7(c).

In our devices, the I $-\mathrm{T}$ characteristics show compliance with both thermal emission and VRH transport mechanisms (see Fig. 7.8) where $\ln (\mathrm{I})$ is linearly dependent on both $\mathrm{T}^{-1}$ and $\mathrm{T}^{-1 / 3}$ possibly due to the relatively small measured temperature range ( 248 K-398 K). However, for the "ideal" Anderson-Mott model, Hall effect measurements should then result in a temperature activated carrier density while the mobility should show a weak temperature dependency (from scattering mechanisms such as involving interface roughness or phonons) [266]. Therefore, our results which on the contrary show a practically temperature independent carrier density (ignoring a variation possibly due to a measurement error) and a mobility with an identical temperature dependence as that of the current (conductivity), cannot be simply explained by the "ideal" AndersonMott model based on short-range potential fluctuations, associated with microscopic inhomogeneities in the material. Similarly, the possibility of VRH transport can be ruled out since it predicts both the Hall mobility and carrier density to be thermally activated $\left(\propto \exp \left(-\left(\mathrm{T}_{0} / \mathrm{T}\right)^{1 / 3}\right)\right)$ as described in [266]. This also explains the unrealistically high characteristic hopping temperature, $\mathrm{T}_{0}$, obtained from the $\mathrm{I}-\mathrm{T}$ curve fitting (see Fig. 7.8(b)). 


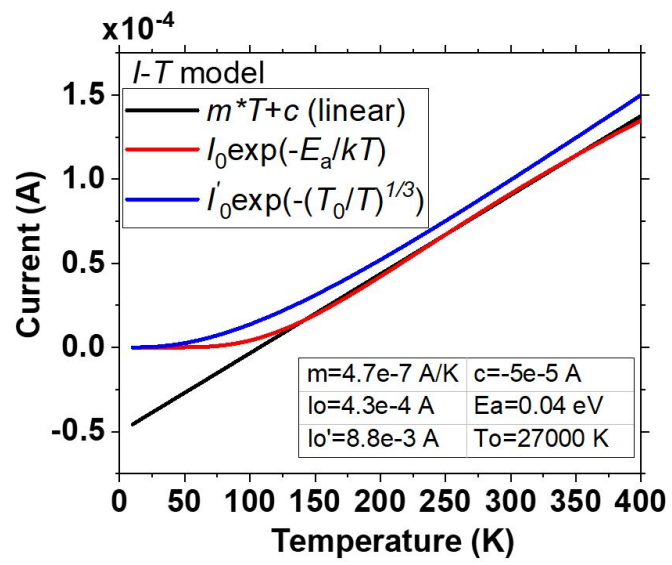

Figure 7.9: Modeled curve for measured I - T dependence (ref. Fig. 7.6) using three different temperature dependent functions i.e. linear, $\exp \left(-E_{a} / k T\right)$ and $\exp \left(-\left(T_{0} / T\right)^{1 / 3}\right)$. Experimentally extracted parameter values were used for all functions.

Similar results have been reported before ([266] and references therein) and are explained using a long-range potential fluctuations (due macroscopic inhomogeneities in the material) model and the percolation theory [268]. According to this model, "insulating" regions $\left(E_{C}>E_{F}\right)$ exist side-byside with "metallic" regions $\left(E_{F}>E_{C}\right)$, as shown graphically in Fig. 7.7(d). In this situation, increasing the carrier density (by shifting $E_{F}$ upwards), makes the "metallic" regions larger and the potential barriers separating them lower. At a certain percolation threshold, which occurs when the fraction of "insulating" area equals the fraction of "metallic" area, a continuous conduction path is established between the "metallic" regions. Above this threshold, thermally activated transport persists where the excitation of carriers over insulating barriers leads to a further reduction in the resistance of the percolation path. The channel conductivity, mobility and their temperature dependence can then be derived using the effective medium theory [268]. In this situation, as described in [266], the conductivity and Hall mobility show the same thermally activated behavior, i.e. $\exp \left(-E_{a} / k T\right)$ dependence. However, the Hall coefficient $\left(R_{H}\right)$ that determines the carrier density $\left(n=1 / q R_{H}\right)$ corresponds to that of the metallic region and therefore is temperature independent.

The long-range fluctuation model with a carrier density above the percolation threshold best describes our results where the current $\left(E_{a}=0.04\right.$ eV, see Fig. 7.8(a)) and Hall mobility $\left(E_{a}=0.02 \mathrm{eV}\right.$, see Fig. 7.2(d)) show similar thermal activation energy, $E_{a}$, while the carrier density is largely temperature independent.

Further, it should be noted that the observed linear as well as the $\exp \left(-\left(\mathrm{T}_{0} / \mathrm{T}\right)^{1 / 3}\right)$ dependence of the measured current with temperature, in addition to proposed $\exp \left(-E_{a} / k T\right)$ dependence, is purely mathematical 


\section{4}

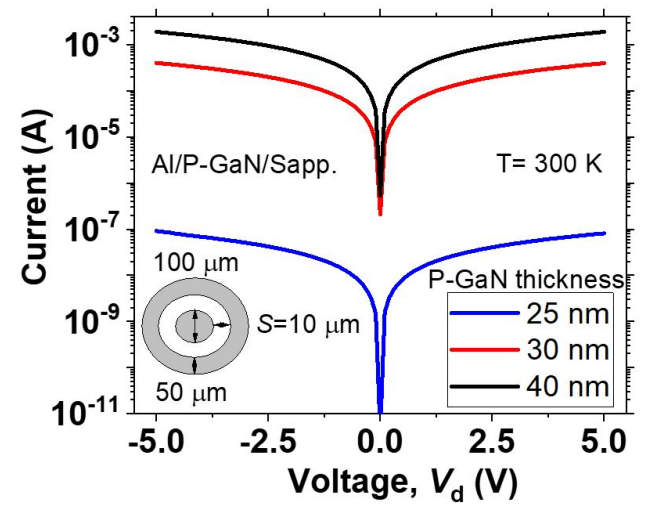

Figure 7.10: I - V characteristics of P-GaN/sapphire devices for varying P-GaN layer thicknesses $(\mathrm{T}=300 \mathrm{~K})$.

in nature. Fig. 7.9 shows that for the extracted parameters in the given measured temperature range, the three functions show practically identical $\mathrm{I}-\mathrm{T}$ relations.

\section{P-GaN layer thickness variation}

In order to understand whether the lateral conduction takes place at the surface or in bulk, electrical measurements on devices for various P-GaN layer thicknesses ${ }^{2}$ were performed, as shown in Fig. 7.10.

All three samples showed linear I - V characteristics however the current level of the $25 \mathrm{~nm}$ sample is at least three orders of magnitude less compared to that of other two ( $30 \mathrm{~nm}$ and $40 \mathrm{~nm}$ ) devices, implying that the current scales, but not linearly, with the thickness. The sheet resistance, $R_{\text {sh }}$, measured using CTLM test structures confirmed this behavior. The measured $R_{\text {sh }}$ neither remained constant with varying thickness nor it scaled linearly making it difficult to distinguish the surface contribution from that of the bulk type of conductance. It appears that the film quality (hence lateral conductance) improved with layer thicknesses. The fact that the I $-\mathrm{V}$ is linear even for a $25 \mathrm{~nm}$ thickness suggests that the electron concentration could be equally high in all three devices while their mobility improved with the thickness due to possible reduction in defect density or grain boundaries that affect the lateral charge transport.

Table 7.2 summarizes the extracted electrical properties of the P-GaN layer on various samples using Hall measurements and circular transmission line measurements (CTLM) [262]. For a nominal layer thickness in the range of 30-40 nm, $R_{\mathrm{sh}} \sim 400-500 \mathrm{k} \Omega / \square$ with specific contact resistivity, $\rho_{\mathrm{c}}$ of $\sim 10^{-3}-10^{-4} \Omega-\mathrm{cm}^{2}$. The transfer length is in the range of $1 \mu \mathrm{m}$ for all measured samples.

\footnotetext{
${ }^{2}$ ALD of poly-GaN films for this experiment were performed by B.Y. van der Wel and R.O. Apaydin.
} 
Table 7.2: Electrical properties of the P-GaN layer at $300 \mathrm{~K}$ for various samples as obtained from Hall analysis. CTLM specifically refers to the data obtained from circular transmission line measurements [262].

\begin{tabular}{|l|l|l|l|l|}
\hline Sample & $\begin{array}{l}\mathrm{n}_{\mathrm{sh}} \\
\left(\mathrm{cm}^{-2}\right)\end{array}$ & $\mathrm{R}_{\mathrm{sh}}(\mathrm{k} \Omega / \square)$ & $\mu_{\mathrm{n}}\left(\mathrm{cm}^{2} / \mathrm{V}-\mathrm{s}\right)$ & $\rho_{\mathrm{c}}\left(\Omega-\mathrm{cm}^{2}\right)$ \\
\hline $\begin{array}{l}\mathrm{Al} / \mathrm{P}- \\
\mathrm{GaN}(40 \\
\mathrm{nm}) / \mathrm{sapphire}\end{array}$ & $10^{14}$ & 433 & 0.16 & -- \\
\hline $\begin{array}{l}\mathrm{Al} / \mathrm{P}- \\
\mathrm{GaN}(20 \\
\mathrm{nm}) / \mathrm{sapphire}\end{array}$ & -- & $440(\mathrm{CTLM})$ & -- & $\begin{array}{l}3.5 \times 10^{-2} \\
(\mathrm{CTLM})\end{array}$ \\
\hline $\begin{array}{l}\mathrm{Al} / \mathrm{P}- \\
\mathrm{GaN}(35 \\
\mathrm{nm}) / \mathrm{SiO}_{2} / \mathrm{Si}\end{array}$ & $10^{14}$ & $\begin{array}{l}68, \quad 185 \\
(\mathrm{CTLM})\end{array}$ & 0.23 & $\begin{array}{l}10^{-4} \\
(\mathrm{CTLM})\end{array}$ \\
\hline $\begin{array}{l}\mathrm{Al} / \mathrm{P}-\mathrm{GaN}(30 \\
\mathrm{nm}) / \mathrm{sapphire}\end{array}$ & -- & $476(\mathrm{CTLM})$ & -- & $\begin{array}{l}6.8 \times 10^{-4} \\
(\mathrm{CTLM})\end{array}$ \\
\hline $\begin{array}{l}\mathrm{Al} / \mathrm{P}-\mathrm{GaN}(40 \\
\mathrm{nm}) / \text { sapphire }\end{array}$ & -- & $98(\mathrm{CTLM})$ & -- & $\begin{array}{l}10^{-5} \\
(\mathrm{CTLM})\end{array}$ \\
\hline
\end{tabular}

\subsubsection{Back-gated P-GaN TFT}

Back-gated P-GaN thin-film transistors (TFTs) (see Fig. 7.11(a)) were also fabricated in order to investigate the effect of field on the channel conductivity. Linear $I_{D S}-V_{D S}$ characteristics were again observed between the drain and source, similar to that observed before for the P-GaN/sapphire devices. The channel conductivity however remains unaffected by the gate voltage, $\mathrm{V}_{\mathrm{GS}}$, as shown in Fig. 7.11(b). A similar conclusion was also drawn
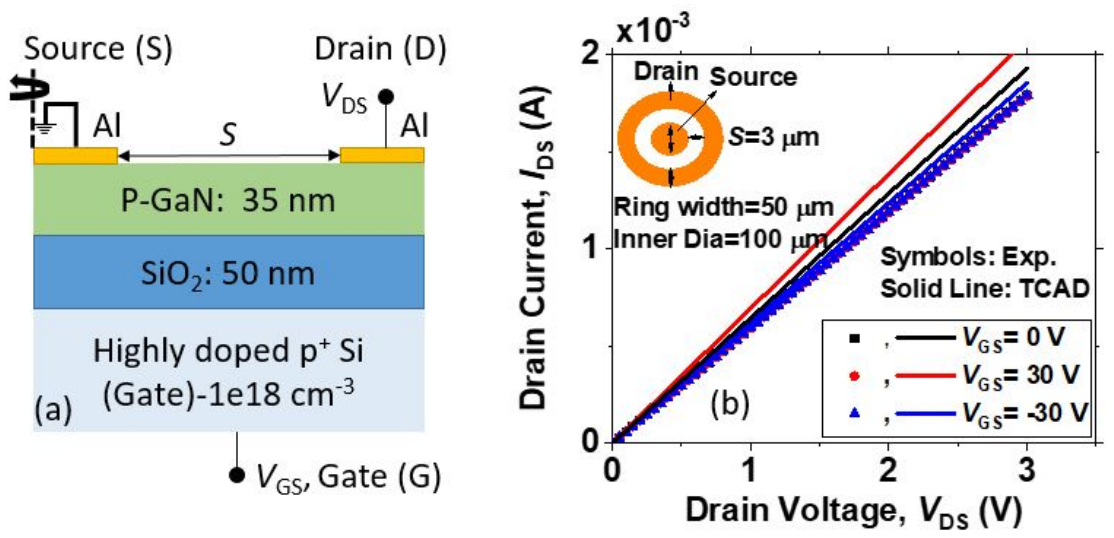

Figure 7.11: (a) Schematic cross-section of the experimental back-gated P-GaN TFT structure, (b) Measured IDS $-V_{D S}$ characteristics for a varied back-gate bias in the same device. 
from measured $I_{D S}-V_{G S}$ characteristics (not shown) where a constant $I_{D S}$ was observed when $V_{G S}$ was swept from $-30 \mathrm{~V}$ to $30 \mathrm{~V}$ at the fixed $V_{D S}=3$ $V$. Moreover, identical results were obtained for different geometries such as rings and rectangular structures and for various drain/source spacings. These observations ascertain the elevated level of carrier density in the P-GaN film which is too high to be depleted by the applied gate bias. Note that the vertical gate leakage for these measurements was in the range of $\sim 10^{-12} \mathrm{~A}$.

To further improve the understanding, TCAD simulations of the device shown in Fig. 7.11(a) were performed. To emulate the possible n-type surface accumulation channel, a high carrier sheet density, $\mathrm{n}_{\mathrm{sh}} \sim 10^{14} \mathrm{~cm}^{-2}$ was incorporated in the top $2 \mathrm{~nm}$ of the GaN film. The rest of the GaN layer was assumed to be lightly n-doped to $10^{15} \mathrm{~cm}^{-3}$. The channel mobility $\left(\mu_{\mathrm{n}}=0.23 \mathrm{~cm}^{2} / \mathrm{V}-\mathrm{s}\right)$ as obtained from the Hall analysis was used. TCAD simulation results (see Fig. 7.11(b)) were found to be in good agreement with the experiments. Moreover they did not show any gate induced field effect for such a high channel density which is in accordance with the experimental observation.

However, similar results could be obtained even if the whole GaN layer would have been uniformly doped with a carrier density $>10^{19} \mathrm{~cm}^{-3}$ instead of having a localized surface channel. Therefore, the actual spatial distribution of the charge carriers inside the P-GaN film, whether accumulated only near the surface or distributed throughout the bulk, cannot be determined from this measurement as well as the TCAD analysis.

\subsection{Electrical properties of T-GaN}

\subsubsection{Lateral (photo-) conduction in T-GaN}

For studying lateral conduction properties of the T-GaN film, three different types of T-GaN devices on sapphire were realized as schematically shown in Fig. 7.12. The first sample had only a T-GaN $(45 \mathrm{~nm})$ film directly on sapphire, the second one had a thin $\operatorname{AlN}(44 \mathrm{~nm})$ layer between the sapphire and T-GaN $(26 \mathrm{~nm})$, while the third had AlN (11 nm) on top of the T-GaN $(31 \mathrm{~nm})$ layer. This was purposefully done to study whether the AlN layer has any effect on the lateral conductance of the GaN layer.

All three devices showed no electrical conduction in the lateral direction under dark condition, see Fig. 7.13(a). Similar conduction behavior has also been observed for $\mathrm{T}-\mathrm{GaN}$ layers grown on $\mathrm{SiO}_{2}$ substrates (not shown). Thermal annealing at $400^{\circ} \mathrm{C}$ for 30 minutes in the $\mathrm{N}_{2}$ environment also did not improve the conduction properties of the T-GaN films. Even measurements at high temperature till $150^{\circ} \mathrm{C}$ did not show any electrical conduction. All T-GaN films, irrespective of the substrate or inclusion of the AIN layer, were found to be electrically insulating in the lateral direction. 

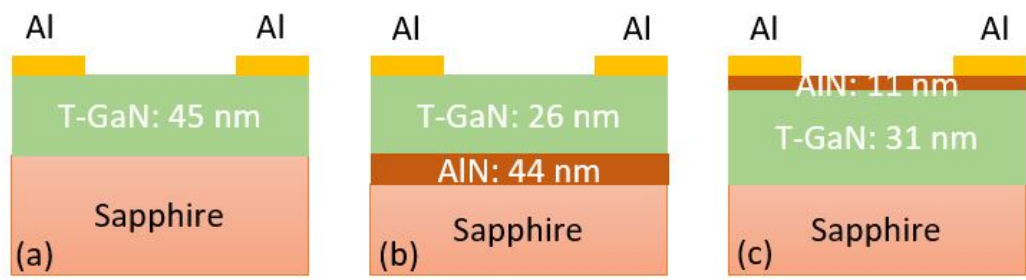

Figure 7.12: Schematic cross-sections of experimental T-GaN devices, (a) TGaN/sapphire, (b) T-GaN/AlN/sapphire, (c) AlN/T-GaN/sapphire.
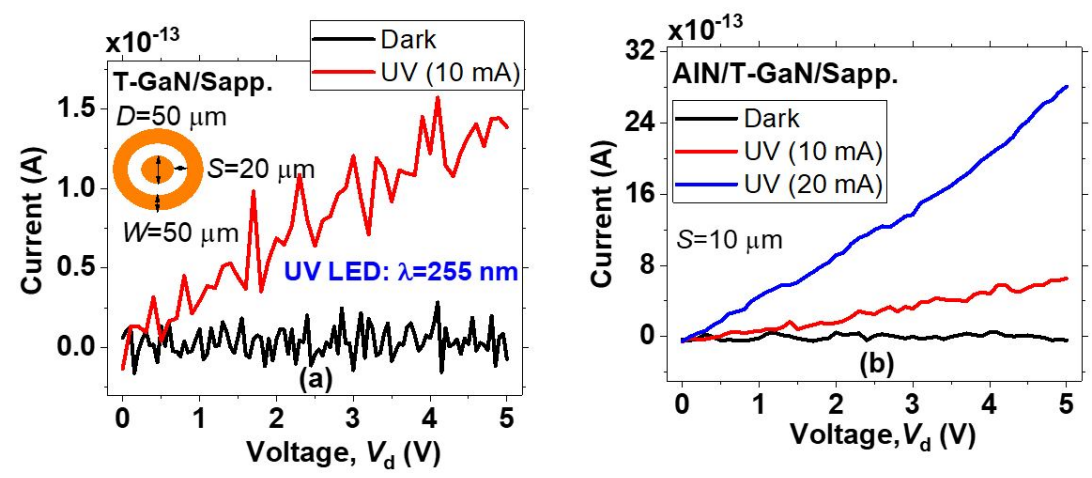

Figure 7.13: I - V characteristics of (a) T-GaN/sapphire and (b) AlN/TGaN/sapphire lateral device samples in dark and under UV illumination condition. An UV LED $(\lambda=255 \mathrm{~nm})$ with a variable optical power was used for illuminating the samples during the measurements $(T=300 \mathrm{~K})$.

The poor lateral conduction in the T-GaN films is likely to be related to its bulk property (i.e. poor carrier concentration and/or high defect density) rather than being limited by the metal-semiconductor contact. Vertical conduction has been observed through a similar Al contacted T$\mathrm{GaN}$ film on $\mathrm{Si}$ with a reverse leakage current of more than $10^{-10} \mathrm{~A}$ (section 7.4.2). The measured lateral current in the range of $10^{-13} \mathrm{~A}(\sim$ noise level of the measurement system) with no clear voltage dependent therefore cannot be explained based on the back-to-back Schottky diode characteristics of the device.

All T-GaN samples grown on sapphire however showed photo-conductive behavior in the presence of a UV LED light source of $255 \mathrm{~nm}$ wavelength (also for $280 \mathrm{~nm}$ ), as shown in Fig. 7.13(a). The optically generated electronhole pairs are responsible for the observed photo-conduction behavior. As expected, the measured photo-current also scales with the optical power (of the UV light source) and is shown for the AlN capped T-GaN sample in Fig. 7.13(b). Moreover, the photo-conduction in the AlN capped sample was three times higher in magnitude compared to that of its counterparts. AlN may be preventing the GaN surface from being oxidized in the air and thereby keeping the intrinsic properties such as the bandgap, of the 

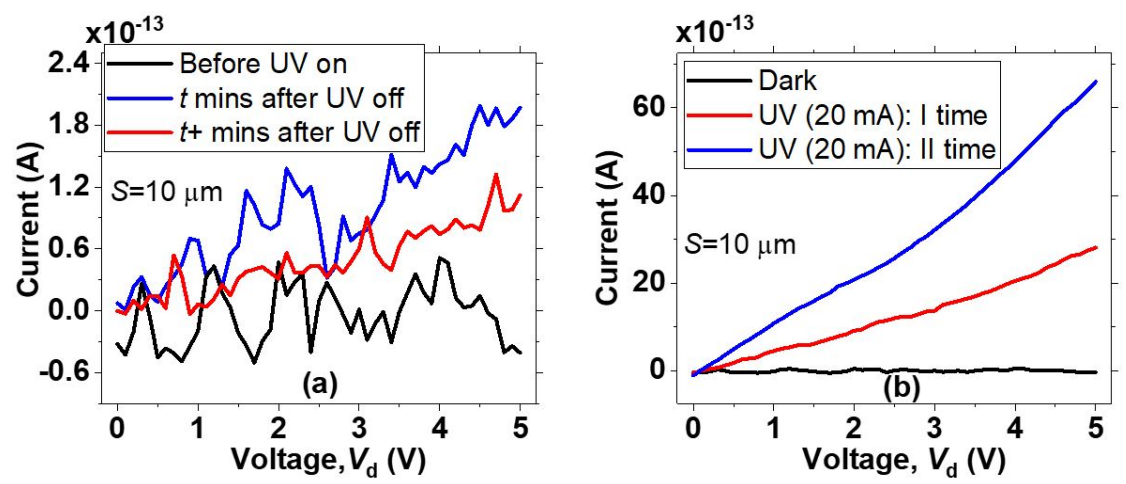

Figure 7.14: Time (history)-dependent I $-\mathrm{V}$ characteristics of the AlN/TGaN/sapphire sample, (a) at different times after switching off the UV light and (b) at different times when the UV light is on $(T=300 \mathrm{~K})$.

as-grown layer preserved. It could also be that the AlN helps in preventing reflection losses at the surface, thereby leading to more light absorption inside the GaN layer. The observed photo-conductance in the T-GaN sample also highlights that the film bulk properties, mainly its low carrier concentration, is responsible for its observed electrically insulating nature in the lateral direction under dark condition.

Further, the samples also showed the persistence photo-conductivity (PPC) effect [269] where the conductivity decays gradually with time and not immediately after switching off the exciting light source, see Fig. 7.14(a). The quick recovery to the initial unperturbed dark state is delayed by the presence of charge carriers (hence current) higher than their steady dark level. Moreover the samples also showed a higher photocurrent when excited for a consecutively second time with UV light before it was fully recovered from the first excitation (see Fig. 7.14(b)). The photo-generated electron-hole pairs remain in the sample for some time after switching off the light source and do not immediately extinct. This phenomenon is common in wide bandgap materials with a high defect density where the probability of the electron capture exceeds that of recombination [269]. This property is however undesirable for photodetector applications where a fast photo response is needed. The time to full recovery or the time between subsequent excitation, though not throughly investigated, was estimated to be in order of few minutes.

\subsubsection{Vertical conduction in T-GaN}

A T-GaN/p-Si diode (see Fig. 7.15(b)) was also realized to examine the vertical electrical conduction properties of the grown T-GaN film. Rectifying I - V characteristics were observed as shown in Fig. 7.15(a). This measurement shows that the T-GaN film, although restricting the lateral conduction, allows the current to flow vertically. The T-GaN/p-Si diode 


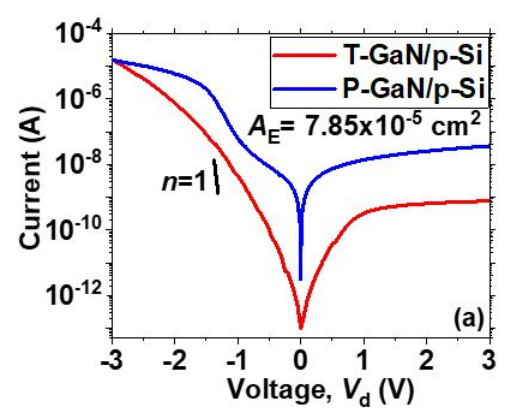

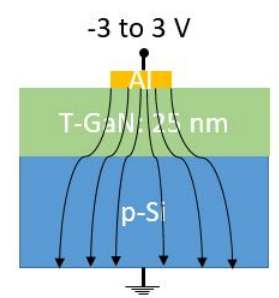

(b)

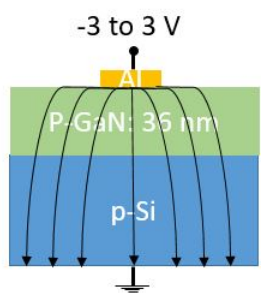

(c)

Figure 7.15: (a) Measured I - V characteristics of the T-GaN/p-Si diode compared to that of the P-GaN/p-Si diode. Schematic cross-section and illustrated current flow lines for, (b) the T-GaN/p-Si diode and (c) the P-GaN/p-Si diode.

also showed higher rectification and a lower leakage current compared to its P-GaN counterpart (refer to chapter 6). Moreover, no peripheral current component $\left(J_{p}\right)$ was observed in case of the former unlike its counterpart where a significant $\mathrm{J}_{\mathrm{p}}$ was measured. The observed lower leakage current in the T-GaN/p-Si diode can be attributed to the absence of $\mathrm{J}_{\mathrm{p}}$.

The schematic cross-section in Figs. 7.15(b)-(c) illustrate the possible current flow lines in both diodes. For the T-GaN/p-Si diode there will be hardly any current spreading from the contact due to the poor lateral conductivity of the T-GaN film. While in the P-GaN/p-Si diode, the current spreads laterally away from the contact (hence significant $\mathrm{J}_{\mathrm{p}}$ ), possibly due to the presence of a surface conductive channel, before entering into the Si substrate. More electrical measurements as discussed below will further clarify the current flow lines illustration as depicted in Figs. 7.15(b)-(c).

In order to further highlight the possible role of a surface conductive channel in the observed electrical properties of the grown P-GaN film, and its comparison to that of T-GaN film, a dedicated test structure comprising two parallel diodes was utilized. In this test structure the first vertical diode $(D-1)$ is surrounded by the second vertical diode outer ring $(D-2)$ as shown schematically in Fig. 7.16(a). The latter, when biased in parallel, acts as a guard ring for the former and thereby inhibit any surface current flow in the region between the two diodes.

For the P-GaN/p-Si device, Fig. 7.16(b) shows the measured vertical current of the $\mathrm{D}-1$ when operated alone $\left(\mathrm{I}_{1}\right)$ and in parallel $\left(\mathrm{I}_{1}^{\prime}\right)$ with $\mathrm{D}-2$. The strong reduction in the current (both forward and reverse) of $D-1$ when operated in parallel with $D-2$ suggest the presence of surface conduction channel between the two diodes which is responsible for the relatively high leakage current. Note that this is even true for a 20 $\mu \mathrm{m}$ spacing between the two diodes. Therefore, the measured difference between $\mathrm{I}_{1}$ and $\mathrm{I}_{1}^{\prime}$ for the P-GaN device can not be explained solely based on the minority current spreading phenomenon, as discussed in chapter 3 using the "2-diodes" measurements. Moreover, the current in D -2 remains 

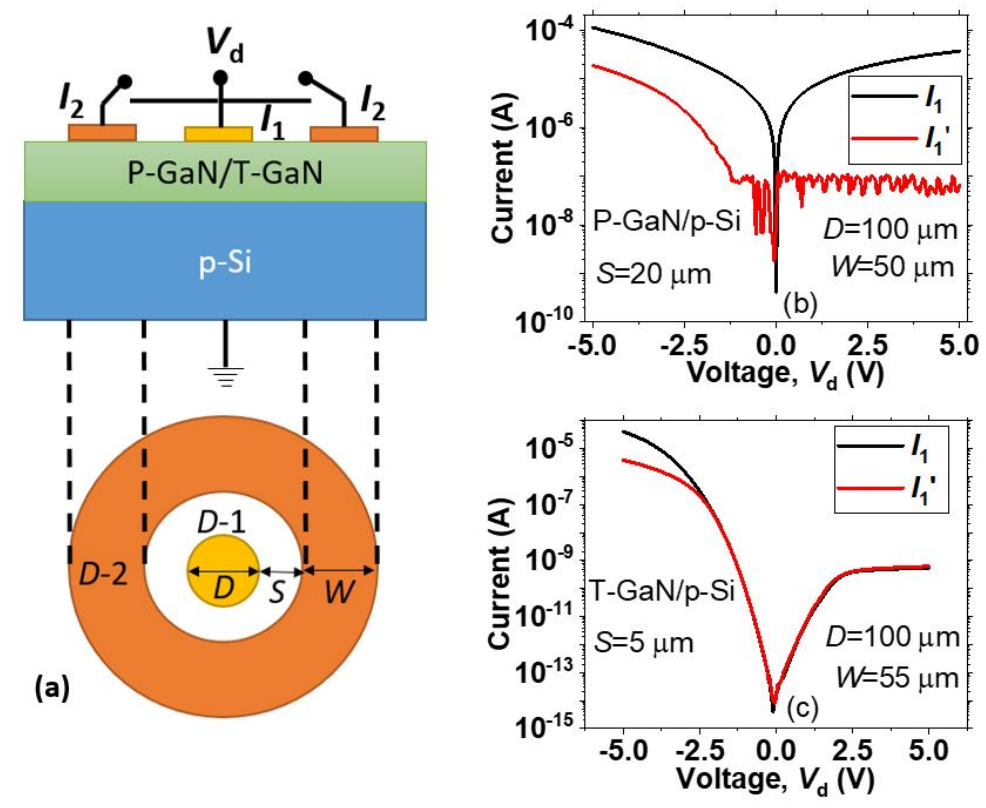

Figure 7.16: (a) Schematic device cross-section and test configuration for measuring the inner diode $(D-1)$ current, with and without connecting outer diode ring $(D-2)$ in parallel. Measured I - V characteristics for, (b) the P-GaN device and (c) the T-GaN device. $I_{1}$ and $I_{1}^{\prime}$ refers to the current through $\mathrm{D}-1$ when the $\mathrm{D}-2$ ring is disconnected and connected in parallel, respectively $(T=300 \mathrm{~K})$.

unaffected irrespective of whether $D-1$ is operating in parallel or not as it remains unguarded in either situation. Note that the poor rectification and the higher leakage current observed on this sample compared to other measurements (ref. to chapter 6) on the same sample is possibly due to its aging with time.

Conversely, no discrepancy is observed between $\mathrm{I}_{1}$ and $\mathrm{I}_{1}^{\prime}$ for the T-GaN diodes. This confirms that there is no dominating surface leakage path and no current spreading in the T-GaN film due to its poor lateral conductivity.

The lateral current transport in a T-GaN/ $\mathrm{p}^{+}-\mathrm{Si}$ structure as shown schematically in Fig. 7.17(a) is examined next. Provided that the metal contacts on top of the T-GaN film do not limit the current, it is expected to scale with the spacing, $\mathrm{S}$, between the contacts but is not influenced by the contact length, L. However the current scales with varying L (see Fig. 7.17(b)) and remains unaffected with a change in $S$ (see Fig. 7.17(c)). The measured non-linear current was limited by the vertical leakage of the heterojunction diode. These measurements indicate that the current first flows vertically through the T-GaN film before crossing the heterojunction to the reach $\mathrm{p}^{+}$-Si region. There it flows laterally (via diffusion) and then finally returns back to the other contact again via the vertical path through the T-GaN film. This current path is indicated using flow lines in Fig. 7.17(a). These results reconfirm that the T-GaN has a poor lateral conductivity and 

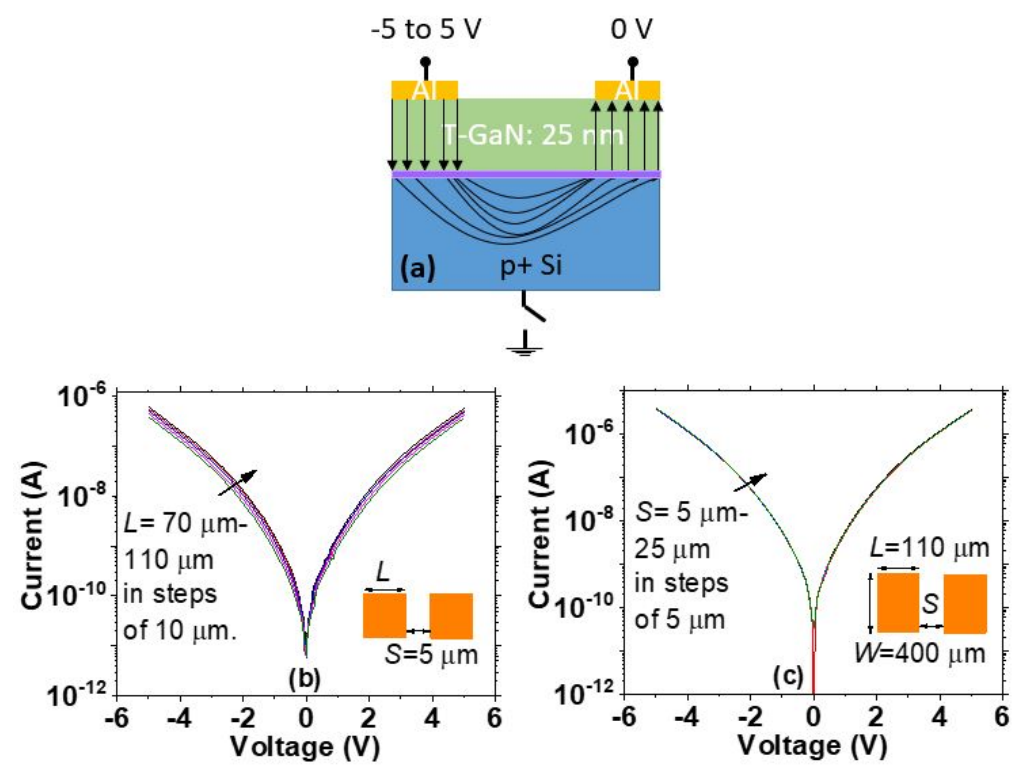

Figure 7.17: (a) Schematic cross-section of the T-GaN/p $\mathrm{p}^{+}-\mathrm{Si}$ structure. Measured $\mathrm{I}-\mathrm{V}$ characteristics of the same structure in the lateral direction, (b) with varied contact length, L and (c) with varied spacing, S, between the contact. The substrate contact was electrically isolated during these measurements.

therefore the current doesn't spread sideways when injected via the contact. Note that, instead of diffusion in bulk $\mathrm{p}^{+}-\mathrm{Si}$, the lateral current inside $\mathrm{p}^{+}-\mathrm{Si}$ could also flow via possible electron inversion layer (ref. Fig. 6.3 in chapter 6) in the $\mathrm{Si}$ at the $\mathrm{GaN} / \mathrm{p}-\mathrm{Si}$ interface.

Conversely, similar measurements on the P-GaN/p-Si structure showed that the current clearly scaled with the contact spacing, but does not change with the contact length, as expected from a lateral current flow via the PGaN film.

\subsection{Discussion}

From the elaborate analysis, it can be concluded that there is high electron density in the P-GaN layer. It is however not established whether these electrons have been distributed across the film thickness or are more concentrated near the surface, though some measurements hint more towards the latter. The observed SCLC limited charge transport in the vertical diode, as discussed in the previous chapter 6, suggests the presence of low electron density $\left(\sim 10^{15} \mathrm{~cm}^{-3}\right)$ in the bulk P-GaN layer. At the same time, the discerned SCLC mechanism also indicates good ohmic contacts (hence high electron density) where the electron injection is not contact limited in the measured voltage range. Both conditions are satisfied by the surface channel model where a high electron density is located near the interface 
promoting an ohmic contact while the electron concentration is relatively low inside the bulk of the film with high trap density. Further, the presence of surface channel could also underline the main reason for the observed high peripheral component, $\mathrm{J}_{\mathrm{p}}$, in the measured diode current.

The observed striking contrast in the lateral conductance of the two types of poly-GaN films could be related to the possible difference in their crystal structure, stoichiometry and/or impurity $(\mathrm{C}, \mathrm{H}, \mathrm{O})$ concentrations. The crystallinity of PEALD films have been known to be generally better than that of thermally-grown films [223]. P-GaN films are also found to be $\mathrm{N}$-rich while T-GaN is more Ga-rich. The observed Ga richness attributed to the accumulation of unreacted TMG-chemisorbed sites, which had actually not converted into GaN, could also possibly explain the poor lateral conductance of the T-GaN layer [223]. Moreover, the higher hydrogen content in the T-GaN layer could also be responsible for its insulating nature.

In addition, the (effective) electron concentration (unintentional doping) in the two films could also be different. The problem of unintentional doping in GaN films has been well articulated by Zhu and Oliver before [270]. It is argued that the role of native defects, such as nitrogen vacancies, (unlike originally thought by early crystal growers) is less important for possible n-type shallow donors due to their high formation energy. This prohibits their spontaneous creation during film growth. On the contrary, the impurities (mainly oxygen) are responsible for the observed n-type conductance in undoped GaN films [270]. Oxygen, can act as a shallow donor by substituting nitrogen from its site, a reaction which is also energetically favorable. Carbon impurity on the other hand is more likely to result in p-type doping as suggested by the first principal calculations. If conductive channel formation is indeed due to the substitution of $\mathrm{N}$ by $\mathrm{O}$, the origin of which is either due to surface oxidation or from the bulk oxygen content, then $\mathrm{O}$ may be substituting $\mathrm{N}$ in somewhat different manner in $\mathrm{P}-\mathrm{GaN}$ and T-GaN. This could arise from differences in their lattice structure, for example. Further study is needed to clarify this.

Previously, PolyGaN based FETs have been reported by several groups [39, 41, 271-273]. Their process often involved doped source/drain junctions, high temperature annealing or a GaN/AlN heterojunction to improve the channel conductivity. High temperature annealing $\left(\sim 800^{\circ} \mathrm{C}\right)$ may also help in improving the T-GaN film conductivity by reducing the defect density and the contact resistance [272] and therefore could be interesting to experiment in the future.

With the present knowledge of the electrical properties of the grown poly-GaN layers presented in this chapter, various potential applications of these materials can be proposed. P-GaN could be attractive for improving the efficiency of Si based IR emitters, or even solar cells without the need of any doped junction, as discussed in the chapter 6 . Further, the lateral (surface) conductive channel in the P-GaN film, if optimized (for gate 
control, channel mobility), could also be promising for FET applications again without requiring any additional doping. A similar concept has been widely exploited in hydrogen-terminated diamond for realizing surface channel FET [274].

T-GaN films can be utilized to realize self isolating vertically functioning devices owing to its poor lateral conductivity. T-GaN films could also be interesting for UV-detection applications as observed from the photoconduction measurements. However, the response time which is plagued by persistence photo-conductivity effect could be of a concern for certain applications. T-GaN could also be attractive for realizing the EHB-LED as proposed in chapter 5 using a combination of high-low gate work functions and AlN as a gate dielectric. Such a dual gate GaN/AlN stack can be easily realized by ALD without breaking the vacuum ensuring good quality interfaces. Further, the polycrystalline nature of the GaN film, though adversely affect light emission, could benefit from its possibly high thermal generation rate as required for the undoped EHB LED operation.

Finally, with regard to possible ED-based poly-GaN devices (switches, LED), P-GaN films in the present form are less suitable as they contain a high density of electron charge which is difficult to deplete or invert either via a Schottky contact or by a applied gate bias. The T-GaN film on the other hand could be interesting for ED-based vertical devices, for e.g. EHB LED as discussed above. Extreme work function contacts such as $\mathrm{MoO}_{\mathrm{x}}$, which appears to be very promising on $\mathrm{Si}$, could also be explored for realizing undoped bipolar devices in poly-GaN material. However more experiments and optimizations of poly-GaN films properties are required to evaluate their true potential for ED based device applications.

\subsection{Conclusion}

An electrical analysis of ALD grown poly-GaN layers was carried out in this chapter. It was found that P-GaN is both vertically and laterally conductive. The vertical conduction in the P-GaN layer is limited by the SCLC mechanism while linear I - V characteristics were obtained in the lateral direction. Hall measurements confirm the presence of a high electron sheet density $\left(\sim 10^{14} \mathrm{~cm}^{-2}\right)$ which was found to be practically independent of the temperature. The Hall mobility $\left(\mu_{n}\right)$ was however found to be temperature dependent with a similar thermal activation as that of conductivity. This observation was explained in the framework of the long-range potential fluctuations model and the percolation theory.

The T-GaN film on the other hand was found to be vertically conductive but laterally insulating under dark condition. The T-GaN film also showed the persistence photoconductivity effect under UV illumination suggesting a low free carrier density and a high trap density inside the layer.

Based on these observed electrical properties of the poly-GaN films various possible applications were proposed. 



\section{CONCLUSIONS AND RECOMMENDATIONS}

In this chapter, general conclusions of the work presented in this thesis are summarized and the key original contributions of this thesis are listed. Finally, recommendations and directions for possible future work are proposed.

\subsection{General conclusions}

Electrostatic doping is a potential solution to the problems connected with chemical doping in UTB devices and emerging semiconductors. With performance at par with conventionally doped counterparts, ED based devices offer additional benefits such as low temperature realization and reconfigurable functionality that could be interesting for future CMOS devices. Moreover ED offers an opportunity to realize innovative device concepts such as EHB-LEDs. Among various reported ED devices, highbarrier Schottky diodes based on extreme work function contacts are a potentially attractive solution for realizing bipolar devices (such as BJTs, LEDs) without adding any doping. Hetero-contacts using extreme work function materials such as $\mathrm{MoO}_{\mathrm{x}}$ offers a possible dopant-free, low temperature route of realizing such high-barrier diodes with bipolar conduction capabilities. Such extreme work function contacts when applied to UTB semiconductors could lead to the formation of localized n-type or $\mathrm{p}$-type regions via volume inversion which can be exploited for various applications.

Thin-film ALD grown poly-GaN on Si is also an interesting technology platform and could open up a new route towards future nanoscale (opto)electronic devices. For example, engineered poly-GaN/Si heterostructures could be a way forward for making efficient optoelectronic devices such 
as IR emitters and solar cells realized in bulk Si. Realization of functional devices based on this material would in general benefit from ED approaches developed in this work, particularly for realizing an n-type region. For p-type ED in such wide bandgap material, high work function materials such as $\mathrm{MoO}_{\mathrm{x}}$ should be explored. Moreover, such contact if optimized can also be employed to lower down the contact resistance of p-type contacts. Further, the EHB-LED concept as proposed in this work could also be utilized in poly-GaN technology platform for light emitting applications.

However, more experiments are needed to access the true merits of poly-GaN thin films and proposed ED solutions.

\subsection{Original contributions of this thesis}

The work presented in this thesis contains several original contributions:

- ED has been previously reported for various material systems and different device geometries. However this approach has never been treated holistically and in a more generalized way using a common theoretical platform. This thesis reviews, defines and discusses the ED approaches for their applicability and adaptability to different material systems and geometries using simple 1-D electrostatics.

- Minority carrier injection in conventional Schottky diodes has been discussed previously, mostly as a high injection phenomenon. This thesis revisits the physics of a high-barrier diodes from an ED perspective and differentiates the operation from conventionally Schottky devices. The modeling/simulation work shows the possibility of using high-barrier Schottky diodes with a high minority-carrier injection ratio as a possible replacement of doped junctions for various bipolar device applications such as light emitters and transistors.

- The high work function property of $\mathrm{MoO}_{\mathrm{x}}$ has previously been reported for realizing a "hole-selective contact" in Si solar cells. However its application to realize an undoped Si diode with bipolar current characteristics was discussed and demonstrated for the first time in this work. Alongside high-barrier property of such contact to $\mathrm{Si}$, the role of $\mathrm{SiO}_{\mathrm{x}}$ interlayer and resulting reduced surface recombination velocity was shown to play a key role in suppressing the electron injection from the substrate. Using such $\mathrm{MoO}_{\mathrm{x}}$ based contacts, bipolar transistor action and light emission were also demonstrated in bulk undoped Si substrates for the first time paving the way forward for undoped transistors and LEDs.

- A one to one comparison between the electrical and optical properties of high-barrier $\mathrm{Pd} / \mathrm{MoO}_{\mathrm{x}} / \mathrm{n}$-Si diodes and implanted $\mathrm{p}^{+} / \mathrm{n} \mathrm{Si}$ reference diodes (realized in the same technology) is presented for the first time in this work. 
- A light emitting device concept based on electrostatically induced electron-hole bilayer in undoped UTB channel semiconductor has been proposed and investigated using TCAD simulations for the first time.

- Polycrystalline GaN based heterojunction diodes to silicon has been realized and studied for the first time in this work. The heterojunction to poly-GaN layer improved the infrared light emission from bulk Si by almost three orders in magnitude. Further, the electrical properties of poly-GaN thin films has also been investigated and analyzed in this work for their possible device applications.

\subsection{Recommendations and future work}

- Electrical measurements on $\mathrm{MoO}_{\mathrm{x}} / \mathrm{n}$-Si diodes reported in this work showed that the electron current is relatively low compared to the hole current but the actual level of the former is still unknown. Experiments on $\mathrm{MoO}_{\mathrm{x}}$ based vertical bipolar transistor test structures in bulk $\mathrm{Si}$ will be needed for individually extracting the electron and hole components of the current. The minority carrier injection ratio, $\gamma$, can be determined from these electrical measurements which would shed more light on the bipolar conduction in such diodes.

- In this work, the proof-of-concept SBT has been demonstrated on a bulk Si substrate with unoptimized device geometry for its ease of experimental realization. However for obtaining a high gain bipolar transistor, such a concept should be tried experimentally on SOI or other UTB semiconductor technology as shown with TCAD simulations in this work.

- The surface inversion in a semiconductor (in our case $\mathrm{Si}$ ) when contacted with extreme work function materials such as $\mathrm{MoO}_{\mathrm{x}}$, was confirmed experimentally from its sheet resistance and diode characteristics. Such contacts when applied to UTB layers could lead to volume inversion. However experiments are needed to assess the true potential of such contacts. Further, the use of proposed $\mathrm{C}-\mathrm{V}$ technique [275] can be explored to obtain the signature of induced ED in UTB devices.

- In this work the EHB-LED concept is proposed and investigated via TCAD simulations. However, experimental realization of such a device is a must to assess its true potential and therefore should be the focus of future work. It will be interesting to investigate the EHB LED based on poly-(In)GaN material for UV/visible light applications. EHB formation in such polycrystalline materials may benefit from possibly high thermal generation rate while light emission in general would profit from its direct bandgap. Moreover, a dual gate stack 
(e.g. AlN/GaN/AlN) as required for EHB LED can be realized using an ALD process without actually breaking the vacuum ensuring high quality interfaces. Further, $\mathrm{MoO}_{\mathrm{x}}$ along with $\mathrm{LiF}_{\mathrm{x}}$ can be explored as high/low work function gate electrodes.

- In this work it was theoretically established that an array of small $\mathrm{nm}$-size Schottky contacts are better light emitters than a single big $\mu \mathrm{m}$-size contact. This concept could be interesting when designing light emitters based on Schottky contacts and therefore should be experimentally investigated in the future.

- Most ED ideas developed in this work have been initially investigated on $\mathrm{Si}$. Such ideas should however be ultimately investigated on (poly) GaN. The EHB-LED is one example proposed above. Other ideas such as $\mathrm{MoO}_{x}$ based (high-)barrier diodes on n-type (intrinsic) $\mathrm{GaN}$ could be interesting for light emission. A similar contact if optimized on p-type (poly-)GaN could help in forming a good ohmic contacts with a low contact resistance.

- Along with the high work function $\mathrm{MoO}_{\mathrm{x}}$ contact for p-type ED, a low work function material such as $\mathrm{Hf}, \mathrm{Cs}$ or even $\mathrm{LiF}_{\mathrm{x}}$ should be explored for n-type ED. An experimental toolbox equipped with such complimentary work function electrodes would be convenient for designing any ED based device application in different semiconductors.

- Other applications of poly-GaN thin films should also be explored. For example, the highly conductive channel in P-GaN should be investigated for FET or even power applications. In general, poly$\mathrm{GaN} / \mathrm{Al}(\mathrm{Ga}) \mathrm{N}$ heterostructures or even their superjunction should be investigated in more detail for polarization induced 2-DEGs and 2-DHGs which was not the focus of this work.

- Last but not the least, adhesion issues (on $\mathrm{GaN}$ ), temperature stability and high resistivity of extreme work function contacts such as $\mathrm{MoO}_{\mathrm{x}}$ should be addressed for reliable technology application. In addition, systematic annealing experiments which were avoided throughout this work should be the part of future work for optimized, reproducible and robust contact properties. 


\section{ELECTRIC FIELD AND CARRIER CONCENTRATION IN THE ELECTRON-HOLE BILAYER SYSTEM}

A case of electrostatically induced electron-hole bilayer (EHB) in dual gated UTB channel is considered and a closed-form analytical expressions for its formation are derived based on 1-D electrostatics. For doing so, quantum mechanical effects as reported before by Trivedi and Fossum [276] for dual gate SOI MOSFETs and more recently, Alper et al. [108] for EHB-TFET systems are neglected.

From Fig. 2.11, it can be written that

$$
\begin{aligned}
& \mathrm{V}_{\mathrm{GT}}=\mathrm{V}_{\mathrm{oxT}}+\Delta \phi_{\mathrm{mT}}+\psi_{\mathrm{sT}} \\
& \mathrm{V}_{\mathrm{GB}}=\mathrm{V}_{\mathrm{oxB}}+\Delta \phi_{\mathrm{mB}}+\psi_{\mathrm{sB}},
\end{aligned}
$$

where $V_{\mathrm{GT}}$ and $\mathrm{V}_{\mathrm{GB}}$ are top and bottom gate voltages, $\mathrm{V}_{\mathrm{oxT}}$ and $\mathrm{V}_{\mathrm{oxB}}$ are voltage drops across top and bottom oxides, $\Delta \phi_{\mathrm{mT}}$ and $\Delta \phi_{\mathrm{mB}}$ are top and bottom gate-body work function difference and $\psi_{\mathrm{sT}}$ and $\psi_{\mathrm{sB}}$ are top and bottom surface potentials. Now applying Gauss' law at both surfaces of the semiconductor body it can be written that

$$
\begin{gathered}
\mathrm{V}_{\mathrm{oxT}}=\frac{1}{\mathrm{C}_{\mathrm{oxT}}}\left(\varepsilon_{\mathrm{s}} \mathrm{E}_{\mathrm{sT}}-\mathrm{Q}_{\mathrm{fT}}-\mathrm{Q}_{\mathrm{itT}}\right) \\
\mathrm{V}_{\mathrm{oxB}}=\frac{1}{\mathrm{C}_{\mathrm{oxB}}}\left(-\varepsilon_{\mathrm{s}} \mathrm{E}_{\mathrm{sT}}-\mathrm{Q}_{\mathrm{fB}}-\mathrm{Q}_{\mathrm{itB}}\right)
\end{gathered}
$$

where $C_{\text {oxT }}$ and $C_{o x B}$ are the top and bottom areal oxide capacitances, $E_{S T}$ is the electric field in the body at the top surface, $\varepsilon_{\mathrm{s}}$ is the semiconductor

The derivations were performed by R.J.E. Hueting. 
dielectric constant, $\mathrm{Q}_{\mathrm{fT}}$ and $\mathrm{Q}_{\mathrm{fB}}$ are fixed charge densities, and $\mathrm{Q}_{\mathrm{itT}}$ and $\mathrm{Q}_{\mathrm{itB}}$ are the interface trapped charge densities at top and bottom surfaces respectively. Since full depletion is assumed, the electric field is constant inside the body:

$$
\psi_{\mathrm{sT}}-\psi_{\mathrm{sB}}=\mathrm{E}_{\mathrm{sT}} \mathrm{t}_{\mathrm{s}}=-\mathrm{E}_{\mathrm{sB}} \mathrm{t}_{\mathrm{s}}
$$

where $t_{s}$ is the thickness of the body and $E_{s B}$ is the electric field in the body at the bottom surface. Now for simplicity sake, neglecting fixed charge at both surfaces and a relatively small inversion charge, it can be written that

$$
\begin{aligned}
& \psi_{\mathrm{sT}}=V_{\mathrm{GT}}-\frac{\varepsilon_{\mathrm{s}} \mathrm{E}_{\mathrm{sT}}}{\mathrm{C}_{\mathrm{oxT}}}-\Delta \phi_{\mathrm{mT}} \\
& \psi_{\mathrm{sB}}=V_{\mathrm{GB}}+\frac{\varepsilon_{\mathrm{s}} \mathrm{E}_{\mathrm{sT}}}{\mathrm{C}_{\mathrm{oxB}}}-\Delta \phi_{\mathrm{mB}} .
\end{aligned}
$$

Subtracting Eq. (A.7) from Eq. (A.6) and replacing the term $\psi_{\mathrm{sT}}-\psi_{\mathrm{sB}}$ with $E_{s T} t_{s}$ and substituting $C_{o x T}=\varepsilon_{o x} / t_{o x T}$ and $C_{o x B}=\varepsilon_{o x} / t_{o x B}$ it can be obtained that

$$
E_{s}=\frac{\left(V_{G T}-V_{G B}\right)-\left(\phi_{m T}-\phi_{m B}\right)}{\frac{\varepsilon_{s}}{\varepsilon_{o x}}\left(t_{o x T}+t_{o x B}\right)+t_{s}} .
$$

The effect of the interface traps on the electrostatics of the EHB system can also be incorporated here. Consider a device in which $t_{\mathrm{oxB}}=t_{\mathrm{oxT}}=t_{\mathrm{ox}}$ with the same uniform interface trap charge density at both semiconductor surfaces, then there is a constant areal interface capacitance $C_{i t}$, and consequently $Q_{i t T}=C_{i t} \cdot \psi_{s T}$ and $Q_{i t B}=C_{i t} \cdot \psi_{s B}$. Then it can be obtained that

$$
E_{\mathrm{s}}=\frac{\left(V_{\mathrm{GT}}-V_{\mathrm{GB}}\right)-\left(\phi_{\mathrm{mT}}-\phi_{\mathrm{mB}}\right)}{\frac{2 \varepsilon_{\mathrm{s}}}{\varepsilon_{\mathrm{ox}}} \cdot t_{\mathrm{ox}}+\left(1+\frac{\mathrm{C}_{\mathrm{it}} \cdot \mathrm{t}_{\mathrm{ox}}}{\varepsilon_{\mathrm{ox}}}\right) \cdot \mathrm{t}_{\mathrm{s}}} .
$$

Hence for the same biasing conditions it can be concluded that the higher the $C_{i t}$, i.e. the higher trap density, the lower the $E_{s}$ and therefore the effectiveness of the EHB concept. 


\section{SOLUTION OF POISSON'S EQUATION IN THE PRESENCE OF INVERSION LAYER}

A high-barrier n-type Schottky diode where a hole inversion layer has formed at the MS interface as shown in Fig. B.1 is considered. Here $\phi(x)$ is the electron potential from the bottom of the conduction band edge $E_{c}$ with boundary conditions $\phi(0)=\phi_{\mathrm{b}}$ and $\phi(\infty)=\phi_{\mathrm{e}}$.

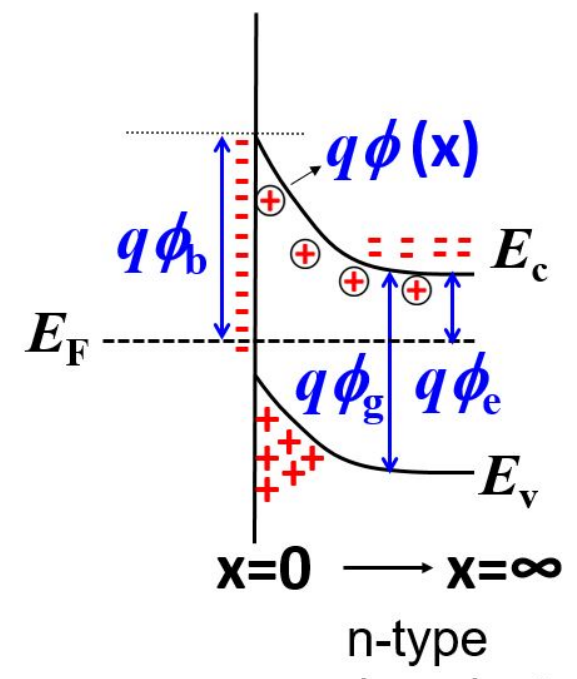

metal semiconductor

Figure B.1: Schematic energy band diagram for a high-barrier n-type Schottky diode [136]. + sign denotes a hole, - sign denotes an electron and circle with a + sign denotes a donor charge. 
By adapting the methodology as outlined in [136] and considering unequal conduction band $\left(\mathrm{N}_{\mathrm{c}}\right)$ and valence band $\left(\mathrm{N}_{\mathrm{v}}\right)$ effective density of states in this work, the electron density, $n$, at thermal equilibrium at any distance $x$ from the interface can be written as:

$$
n(x)=N_{d} \exp \left(\frac{-\left(\phi(x)-\phi_{e}\right)}{u_{T}}\right) .
$$

Similarly, assuming Boltzmann's approximation, the hole density, can be written as:

$$
p(x)=\frac{n_{i}^{2}}{n(x)}=\frac{n_{i}^{2}}{N_{d}} \exp \left(\frac{\phi(x)-\phi_{e}}{u_{T}}\right),
$$

where $n_{i}$ is the intrinsic carrier concentration, and $u_{T}$ is the thermal voltage $(=\mathrm{kT} / \mathrm{q}$, where $\mathrm{k}$ is Boltzmann's constant, $\mathrm{T}$ is the temperature and $\mathrm{q}$ is the elementary charge).

At any applied voltage, $V_{d}$ :

$$
n(x)=N_{d} \exp \left(\frac{-\left(\phi(x)-\phi_{e}-v_{d}\right)}{u_{T}}\right),
$$

and

$$
p(x)=\frac{n_{i}^{2}}{N_{d}} \exp \left(\frac{\phi(x)-\phi_{e}}{u_{T}}\right) .
$$

Note that $p(x)$ is independent of $V_{d}$ in the space charge region as it is determined by $\phi_{\mathrm{b}}$ which is constant. For such an MS junction, the 1-D Poisson's equation can be written as:

$$
\begin{aligned}
\frac{\mathrm{d}^{2} \phi}{\mathrm{d} x^{2}} & =\frac{\mathrm{q}\left[N_{d}+p(x)-n(x)\right]}{\epsilon_{\mathrm{s}}} \\
& =\frac{q}{\epsilon_{\mathrm{s}}}\left[N_{\mathrm{d}}+\frac{n_{\mathrm{i}}^{2}}{\mathrm{~N}_{\mathrm{d}}} \exp \left(\frac{\phi(x)-\phi_{\mathrm{e}}}{\mathrm{u}_{\mathrm{T}}}\right)-\mathrm{N}_{\mathrm{d}} \exp \left(\frac{-\left(\phi(x)-\phi_{\mathrm{e}}-\mathrm{V}_{\mathrm{d}}\right)}{\mathrm{u}_{\mathrm{T}}}\right)\right] \\
& =\frac{\mathrm{q} N_{\mathrm{d}}}{\epsilon_{\mathrm{s}}}\left[1+\frac{\mathrm{n}_{\mathrm{i}}^{2}}{\mathrm{~N}_{\mathrm{d}}^{2}} \exp \left(\frac{\phi(x)-\phi_{\mathrm{e}}}{\mathrm{u}_{\mathrm{T}}}\right)-\exp \left(\frac{-\left(\phi(x)-\phi_{\mathrm{e}}-\mathrm{V}_{\mathrm{d}}\right)}{\mathrm{u}_{\mathrm{T}}}\right)\right]
\end{aligned}
$$

Now, lets define

$$
\frac{\mathrm{q} N_{\mathrm{d}}}{\epsilon_{\mathrm{s}}}\left[1+\frac{\mathrm{n}_{\mathrm{i}}^{2}}{\mathrm{~N}_{\mathrm{d}}^{2}} \exp \left(\frac{\phi(x)-\phi_{\mathrm{e}}}{\mathrm{u}_{\mathrm{T}}}\right)-\exp \left(\frac{-\left(\phi(x)-\phi_{\mathrm{e}}-\mathrm{V}_{\mathrm{d}}\right)}{\mathrm{u}_{\mathrm{T}}}\right)\right]=\mathrm{F}(\phi)
$$

and

$$
\frac{\mathrm{d} \phi}{\mathrm{dx}}=\mathrm{E}(\phi)
$$

Therefore,

$$
\begin{aligned}
\frac{d E}{d x} & =F(\phi), \\
d E & =F(\phi) d x \\
& =\frac{F(\phi) d \phi}{d \phi / d x},
\end{aligned}
$$


and

$$
E d E=F(\phi) d \phi .
$$

Integrating Eq. (B.10) on both sides

$$
\int E d E=\int F(\phi) d \phi
$$

yields

$$
\frac{E^{2}}{2}+C_{1}=f(\phi)+C_{2}
$$

where $f(\phi)$ is the anti-derivative of $F(\phi)$ and $C_{1}$ and $C_{2}$ are constants of integration. Using the boundary condition that $\mathrm{d} \phi / \mathrm{dx}=\mathrm{E}=0$, at $\phi=\phi_{\mathrm{e}}+$ $\mathrm{V}_{\mathrm{d}}$, it can be obtained that

$$
C_{1}-C_{2}=f\left(\phi_{e}+V_{d}\right) .
$$

Combining Eqs. (B.7), (B.12) and (B.13) it can be obtained that

$$
\frac{1}{2}\left(\frac{d \phi}{d x}\right)^{2}=f(\phi)-f\left(\phi_{e}+V_{d}\right) .
$$

After taking the square root and integrating on both sides, Eq. (B.14) can be rearranged as

$$
\int_{0}^{x} d x=\int_{\phi_{b}}^{\phi} \frac{d \phi}{\sqrt{2\left(f(\phi)-f\left(\phi_{e}+V_{d}\right)\right)}} .
$$

Now, using Eq. (B.6)

$f(\phi)=\frac{q N_{d}}{\epsilon_{s}}\left[\phi+\frac{n_{i}^{2}}{N_{d}^{2}} u_{T} \exp \left(\frac{\phi(x)-\phi_{e}}{u_{T}}\right)+u_{T} \exp \left(\frac{-\left(\phi(x)-\phi_{e}-V_{d}\right)}{u_{T}}\right)\right]$

and

$$
f\left(\phi_{e}+V_{d}\right)=\frac{q N_{d}}{\epsilon_{s}}\left[\phi_{e}+V_{d}+\frac{n_{i}^{2}}{N_{d}^{2}} u_{T} \exp \left(\frac{V_{d}}{u_{T}}\right)+u_{T}\right] .
$$

Therefore, integrating Eq. (B.15) yields

$$
x=\int_{\phi_{\mathrm{b}}}^{\phi} \frac{\mathrm{d} \phi}{\sqrt{2\left(\mathrm{f}(\phi)-\mathrm{f}\left(\phi_{\mathrm{e}}+\mathrm{V}_{\mathrm{d}}\right)\right)}} .
$$

The shape of barrier or plot of $\phi$ as a function of distance $x$ can be obtained by numerical integration of Eq. (B.18) after inserting $f(\phi)$ from Eq. (B.16) and $f\left(\phi_{e}+V\right)$ from Eq. (B.17).

The capacitance of the diode can be obtained from calculating the change in the number of electrons in the conduction band with change in the applied bias and therefore can be expressed as follows [136]:

$$
C=q \frac{d N}{d V_{d}},
$$


where $\mathrm{N}$ represent the change in the number of electrons in the conduction band with the applied bias which is equivalent of calculating the total number of unneutralized donors in the conduction band and can be expressed as:

$$
N=\int_{0}^{\infty}\left(N_{d}-n\right) d x
$$

Now, using $n$ from Eq. (B.3)

$$
\left(N_{d}-n\right)=N_{d}\left(1-\exp \left(\frac{-\left(\phi(x)-\phi_{e}-V_{d}\right)}{u_{T}}\right)\right) \text {. }
$$

Therefore,

$$
\begin{aligned}
C & =q \frac{d N}{d V_{d}}=q N_{d} \frac{d}{d V_{d}} \int_{0}^{\infty}\left[1-\exp \left(\frac{-\left(\phi(x)-\phi_{e}-V_{d}\right)}{u_{T}}\right)\right] d x \\
& =q N_{d} \frac{d}{d V_{d}} \int_{0}^{\infty}\left[1-\exp \left(\frac{-\left(\phi(x)-\phi_{e}-V_{d}\right)}{u_{T}}\right)\right] \frac{d \phi}{d \phi / d x} \\
& =q N_{d} \frac{d}{d V_{d}} \int_{\phi_{e}+V_{d}}^{\phi_{b}} \frac{\left[1-\exp \left(\frac{-\left(\phi(x)-\phi_{e}-V_{d}\right)}{u_{T}}\right)\right]}{\sqrt{2\left(f(\phi)-f\left(\phi_{e}+V_{d}\right)\right)}} d \phi .
\end{aligned}
$$

The capacitance of the diode as a function of applied bias can therefore be numerically computed using Eq. (B.22). 


\section{DERIVATION OF CHARGE \\ CARRIERS THERMAL GENERATION \\ TIME}

In the absence of impact ionization and under dark conditions, the carrier generation will be mainly via thermal processes. The thermal generation rate can be distinguished by four processes: bulk space-charge (scr) generation $\left(\mathrm{G}_{1}\right)$, band-to-band generation $\left(\mathrm{G}_{2}\right)$, quasi-neutral bulk generation $\left(\mathrm{G}_{3}\right)$ and surface generation $\left(G_{4}\right) . G_{1}$ is characterized by the generation time $\left(\tau_{\mathrm{g}}\right)$ [262] and $\mathrm{G}_{2}$ is characterized by the radiative recombination coefficient $\left(B_{\text {rad }}\right)$ which is equally important for direct bandgap semiconductors. For the UTB based EHB concept $\mathrm{G}_{3}$ can be ignored. Further, ideal interfaces are assumed here hence surface generation $\left(\mathrm{G}_{4}\right)$ is absent.

For convenience sake, lets focus on a 1-D system with uniform concentrations. Using the continuity equation for electrons (ignoring holes) it can be written [262]:

$$
\frac{\mathrm{dn}}{\mathrm{dt}}=\mathrm{G}=\mathrm{G}_{1}+\mathrm{G}_{2}
$$

where $\mathrm{G}$ is the net generation rate due to various mechanisms.

For bulk scr generation when $\mathrm{pn}<\mathrm{n}_{\mathrm{i}}^{2}$, which is actually the inverse of $\mathrm{SRH}$ recombination, the generation rate can be expressed as [262]:

$$
\mathrm{G}_{1}=-\mathrm{R}_{\mathrm{SRH}}=\frac{\mathrm{n}_{\mathrm{i}}^{2}}{\tau_{\mathrm{p}} n_{1}+\tau_{\mathrm{n}} \mathrm{p}_{1}}=\frac{\mathrm{n}_{\mathrm{i}}}{\tau_{\mathrm{g}}}
$$

Assuming SRH lifetimes $\tau_{\mathrm{n}}=\tau_{\mathrm{p}}=\tau_{\mathrm{SRH}}$ and defect level $\left(\mathrm{E}_{\mathrm{T}}\right)$ coinciding with the intrinsic Fermi level ( $\left.E_{\mathrm{FI}}\right)$, Eq. (C.2) can be re-written as:

$$
\mathrm{G}_{1}=\frac{\mathrm{n}_{\mathrm{i}}}{2 \tau_{\mathrm{SRH}}}
$$

The derivations were performed by R.J.E. Hueting. 
The band-to-band generation can be expressed as:

$$
\mathrm{G}_{2}=\frac{\mathrm{n}}{\tau_{\mathrm{rad}}}=\mathrm{B}_{\mathrm{rad}} \mathrm{n}_{\mathrm{i}}^{2}
$$

So, substituting Eqs. (C.3)-(C.4) in Eq. (C.1), it can be written that:

$$
\frac{\mathrm{dn}}{\mathrm{dt}}=\frac{\mathrm{n}_{\mathrm{i}}}{2 \tau_{\mathrm{SRH}}}+\mathrm{B}_{\mathrm{rad}} \mathrm{n}_{\mathrm{i}}^{2} .
$$

Now, the number of electrons, $n_{0}$, generated in the total time $t_{\text {th }}$ can be determined as:

$$
n_{0}=\int_{0}^{t_{\text {th }}} G d t=\int_{0}^{t_{\text {th }}}\left(\frac{n_{i}}{2 \tau_{S R H}}+B_{\text {rad }} n_{i}^{2}\right) d t .
$$

In principle the generation components do not have a strong time dependence, and consequently:

$$
t_{\text {th }}=\frac{n_{0}}{\left(\frac{n_{i}}{2 \tau_{\text {SRH }}}+B_{\text {rad }} n_{i}^{2}\right)} .
$$

From the above simplified expression, it can be concluded that the generation time $t_{\text {th }}$ rises exponentially with the bandgap through $n_{i}$ and therefore narrow (and direct) bandgap materials are more effective for the thermally generated EHB concept. 


\section{APPENDIX}

\section{INVERSION LAYER IN HIGH-BARRIER PD/ $\mathrm{MOO}_{\chi} / \mathrm{N}-\mathrm{SI}$ DIODES}

The systematic electrical investigation of $\mathrm{Pd}$ capped $\mathrm{MoO}_{x}$ contacts on $\mathrm{Si}$, both n-type and p-type, using dedicated ring-shaped test structures has been reported before in [169]. On those test structures, inversion layer went undetected as current choose to flow via top Pd layer instead of inversion layer. In order to satisfactorily establish the presence of the hole inversion layer at the $\mathrm{MoO}_{x} / \mathrm{n}$-Si interface and to measure its sheet resistance, insulating capping layer is more beneficial instead of conductive layer. For this purpose, another sample with similar test structures was prepared in which conductive Pd capping layer was substituted with a thin insulating boron (B) layer of $\sim 10 \mathrm{~nm}$ following the same (in-situ $\mathrm{B} / \mathrm{MoO}_{x}$ e-beam deposition) procedure as described before [169]. For boron, 99.5 $\%$ pure material from Kurt J. Lesker in the form of cylindrical ingots was utilized. No thermal annealing was performed on any of the prepared samples.

A series of electrical measurements were performed on boron capped $\mathrm{MoO}_{x}$ test structures to check whether there is lateral conduction via the inversion layer. In this case, contact to the inversion layer is made via doped regions after scratching away the few $\mathrm{nm}$ of boron layer from top (see inset of Fig. D.1). Unlike on Pd capped samples, clear downscaling of measured resistance was observed with decreasing ring width confirming the lateral conduction via inversion layer.

The inversion layer sheet resistance was extracted using differential measurements on ring structures to exclude the contact resistance using

\footnotetext{
This appendix is supplementary to the work presented at the $32^{\text {nd }}$ IEEE International Conference on Microelectronic Test Structures (ICMTS), 2019 [169]. The device fabrication for this work was performed by D.T. Shivakumar.
} 
the methodology as described in $[277,278]$. The sheet resistance, $R_{\text {sh }}$, can be estimated as [277, 278]:

$$
R_{s h}=\frac{R_{i j}}{\alpha_{i j}}=\frac{R_{m j}-R_{m i}}{\alpha_{i j}},
$$

where $R_{\mathrm{ij}}$ is the differential resistance between two ring structures of indices $i / j$ with different ring width, $L_{i / j} . R_{m i / j}$ is the measured resistance across the ring and $\alpha_{i j}$ is the coefficient associated between ring pair $i-j$ and can be expressed as:

$$
\alpha_{i j}=\frac{1}{2 \pi} \ln \left(\frac{\left(r_{g}-\frac{1}{2} L_{i}\right) \cdot\left(r_{g}+\frac{1}{2} L_{j}\right)}{\left(r_{g}+\frac{1}{2} L_{i}\right) \cdot\left(r_{g}-\frac{1}{2} L_{j}\right)}\right),
$$

where $r_{g}$ is the ring radius which is fixed and equal to $164 \mu \mathrm{m}$. Using, Eqs. D.1-D.2, the sheet resistance of inversion layer is extracted as shown in Fig. D.1. From measurements across different ring pairs (shown for only 6 pairs), $R_{m i / j}$ in the range of $60-100 \mathrm{k} \Omega / \square$ was extracted. Similar measurements on $\mathrm{p}$-substrate (with $\mathrm{n}^{+}$doped regions) did not showed any conductance which further confirms that the measured resistance is indeed associated with the hole inversion layer. Moreover, the sheet resistance of as deposited boron layer is around $1 \mathrm{M} \Omega / \square$ and therefore doesn't contribute to the measured sheet resistance.

Table D.1 summarizes the results of electrical measurements from [169] and their comparison to values reported in literature. The investigation of the conductance along the Si surface on $\mathrm{B} / \mathrm{MoO}_{x} / \mathrm{n}-\mathrm{Si}$ test structures did reveal the presence of a hole inversion layer as has been reported in the literature albeit with relatively high sheet resistance in the range of $60-100 \mathrm{k} \Omega / \square$. The extracted sheet resistance of inversion layer in the

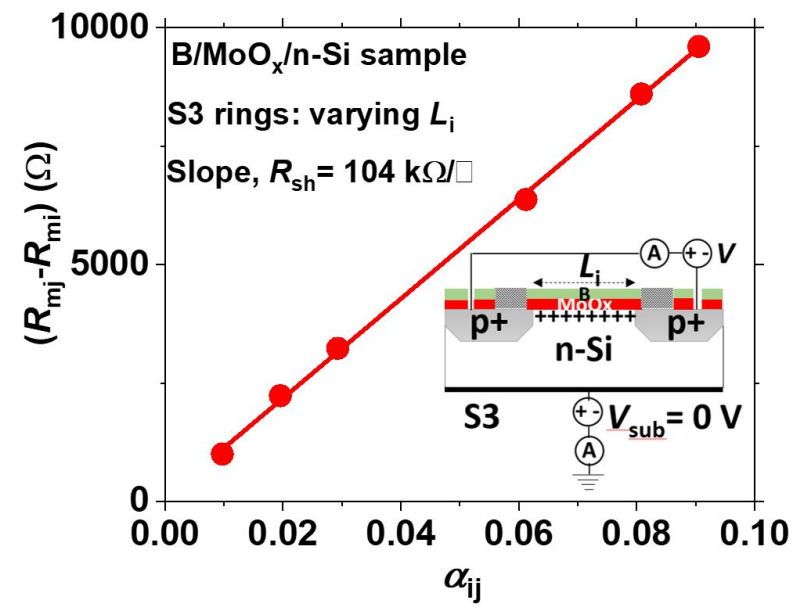

Figure D.1: Extraction of inversion layer sheet resistance using differential resistance measurements of lateral S3 ring structures with various ring widths, $\mathrm{L}_{\mathrm{i}}$. 
Table D.1: Electrical properties of the $\mathrm{Pd} / \mathrm{MoO}_{x}$ stack on silicon as extracted from the measurements on ring-shaped test structures [169] and compared to literature values. Inversion layer sheet resistance reported here is extracted from the measurement on $\mathrm{B} / \mathrm{MoO}_{x} / \mathrm{n}$-Si test structures instead.

\begin{tabular}{|l|l|l|l|l|}
\hline & $\begin{array}{l}\text { contact } \\
\text { resistivity, } \\
\mathbf{P d} / \mathbf{M o O}_{x} \\
\text { to p-Si, } \\
\rho_{\mathrm{c}}\left(\Omega-\mathbf{c m}^{2}\right)\end{array}$ & $\begin{array}{l}\text { resistivity, } \\
\mathbf{M o O}_{x} \rho_{\text {MoOx }} \\
(\Omega-\mathrm{cm})\end{array}$ & $\begin{array}{l}\text { sheet re- } \\
\text { sistance, } \\
\text { inversion } \\
\text { layer } \mathrm{R}_{\text {sh-inv }} \\
(\mathbf{k} \Omega / \square)\end{array}$ & $\begin{array}{l}\text { sheet resis- } \\
\text { tance, p } \\
\text { implant }\end{array}$ \\
$\begin{array}{l}\mathrm{R}_{\text {sh-p+ }} \\
(\Omega / \square)\end{array}$ \\
\hline $\begin{array}{l}\text { our work } \\
{[169]}\end{array}$ & $1.2 \times 10^{-3}$ & $1.5 \times 10^{4}$ & $60-100$ & 25 \\
\hline $\begin{array}{l}\text { literature } \\
\text { values }\end{array}$ & $\begin{array}{l}1.0 \times 10^{-3} \\
{[174]}\end{array}$ & $5 \times 10^{4}[174]$ & $12[174]$ & $25[278]$ \\
\hline
\end{tabular}

range of $60-100 \mathrm{k} \Omega / \square$ is in contrast to the reported sheet resistance value of $\sim 12 \mathrm{k} \Omega / \square$ in [174]. High sheet resistance value indicates that either the $\mathrm{MoO}_{x} / \mathrm{Si}$ bonding structure that supposedly is responsible for the inversion of the n-Si interface only occurs in electrically separated islands, or if the interface has high defect density that seriously deteriorates the carrier mobility. Both explanations are consistent with the fact that the hole injection from the MoOx into the $\mathrm{Si}$ is similar to that measured on fullyimplanted $\mathrm{p}^{+}$-n diodes. Further, the role of the thermal annealing step, which has not been explored in our work due to the known temperature instability of $\mathrm{MoO}_{x}$ [193-195], could also be interesting for a future study. Finally, though $\mathrm{MoO}_{x}$ readily forms an ohmic contact to $\mathrm{p}$-Si without any thermal annealing step, the contact resistivity of $\mathrm{Pd} / \mathrm{MoO}_{x} / \mathrm{Si}$ stack as also reported before [174] is too high for any device application. The contact resistivity, $\rho_{\mathrm{c}}=1.2 \mathrm{~m} \Omega-\mathrm{cm}^{2}$, was measured on the p-Si interface which could be an order of magnitude higher on the n-Si interface [174] making it suitable only for large area devices such as solar cells. 



\section{BIBLIOGRAPHY}

[1] S. M. Sze and K. K. Ng, Physics of semiconductor devices, 3rd ed. John wiley \& sons, 2007.

[2] E. C. Jones and E. Ishida, "Shallow junction doping technologies for ULSI," Materials Science and Engineering R Reports, vol. 24, no. 1, pp. 1 - 80, Oct. 1998.

[3] P. S. Peercy, "The drive to miniaturization," Nature, vol. 406, no. 6799, pp. 1023-1026, 2000.

[4] X. Tang, V. K. De, and J. D. Meindl, “Intrinsic MOSFET parameter fluctuations due to random dopant placement," IEEE Transactions on Very Large Scale Integration (VLSI) Systems, vol. 5, no. 4, pp. 369-376, Dec 1997.

[5] A. Asenov, "Random dopant induced threshold voltage lowering and fluctuations in sub-0.1 um MOSFET's: A 3-D "atomistic" simulation study," IEEE Transactions on Electron Devices, vol. 45, no. 12, pp. 2505-2513, Dec 1998.

[6] B. Cheng, S. Roy, and A. Asenov, "The impact of random doping effects on CMOS SRAM cell," in SolidState Circuits Conference, 2004. ESSCIRC 2004. Proceeding of the 30th European, Sept 2004, pp. 219-222.

[7] C. Thelander, P. Agarwal, S. Brongersma, J. Eymery, L.-F. Feiner, A. Forchel, M. Scheffler, W. Riess, B. Ohlsson, U. Gösele, and L. Samuelson, "Nanowire-based one-dimensional electronics," Materials today, vol. 9, no. 10, pp. 28-35, 2006.

[8] J. Wallentin and M. T. Borgström, “Doping of semiconductor nanowires," Journal of Materials Research, vol. 26, no. 17, pp. 2142-2156, 2011.

[9] W. Walukiewicz, "Intrinsic limitations to the doping of wide-gap semiconductors," Physica B Condensed Matter, vol. 302-303, pp. 123 - 134, Jan. 2001.

[10] P. Kozodoy, H. Xing, S. P. DenBaars, U. K. Mishra, A. Saxler, R. Perrin, S. Elhamri, and W. C. Mitchel, “Heavy doping effects in Mg-doped GaN," Journal of Applied Physics, vol. 87, no. 4, pp. 1832-1835, Feb. 2000.

[11] V. Heera, D. Panknin, and W. Skorupa, "p-Type doping of SiC by high dose Al implantation-problems and progress," Applied Surface Science, vol. 184, no. 1-4, pp. 307 -316, Dec. 2001.

[12] Ü. Özgür, Y. I. Alivov, C. Liu, A. Teke, M. Reshchikov, S. Doğan, V. Avrutin, S.-J. Cho, and H. Morkoç, "A comprehensive review of ZnO materials and devices," Journal of Applied Physics, vol. 98, no. 4, p. 11, 2005.

[13] P. Ayala, R. Arenal, M. Rummeli, A. Rubio, and T. Pichler, "The doping of carbon nanotubes with nitrogen and their potential applications," Carbon, vol. 48, no. 3, pp. 575 - 586, Mar. 2010.

[14] G. Fiori, F. Bonaccorso, G. Iannaccone, T. Palacios, D. Neumaier, A. Seabaugh, S. K. Banerjee, and L. Colombo, "Electronics based on two-dimensional materials," Nature Nanotechnology, vol. 9, no. 10, pp. 768-779, Oct. 2014.

[15] D. Sarkar, X. Xie, W. Liu, W. Cao, J. Kang, Y. Gong, S. Kraemer, P. M. Ajayan, and K. Banerjee, “A subthermionic tunnel field-effect transistor with an atomically thin channel," Nature, vol. 526, no. 7571, pp. 91-95, 2015.

[16] S. Datta, H. Liu, and V. Narayanan, "Tunnel FET technology: A reliability perspective," Microelectronics Reliability, vol. 54, no. 5, pp. 861-874, 2014.

[17] R. Antonov and A. Johnson, "Subband population in a single-wall carbon nanotube diode," Physical Review Letters, vol. 83, no. 16, p. 3274, 1999.

[18] S. Strite and H. Morkoç, "GaN, AlN, and InN: a review," Journal of Vacuum Science E Technology B Microelectronics and Nanometer Structures Processing, Measurement, and Phenomena, vol. 10, no. 4, pp. 1237-1266, 1992.

[19] L. F. Eastman and U. K. Mishra, “The toughest transistor yet [GaN transistors]," IEEE spectrum, vol. 39, no. 5, pp. 28-33, 2002.

[20] H. Morkoç, Handbook of nitride semiconductors and devices, Materials Properties, Physics and Growth. John Wiley \& Sons, 2009, vol. 1.

[21] D. Zhu, D. Wallis, and C. Humphreys, "Prospects of III-nitride optoelectronics grown on Si," Reports on Progress in Physics, vol. 76, no. 10, p. 106501, 2013. 
[22] M. Ishida, T. Ueda, T. Tanaka, and D. Ueda, "GaN on Si technologies for power switching devices," IEEE Transactions on Electron Devices, vol. 60, no. 10, pp. 3053-3059, 2013.

[23] A. Dadgar, "Sixteen years GaN on Si," Physica Status Solidi (b), vol. 252, no. 5, pp. 1063-1068, 2015.

[24] S. Jain, M. Willander, J. Narayan, and R. V. Overstraeten, “III-nitrides: Growth, characterization, and properties," Journal of Applied Physics, vol. 87, no. 3, pp. 965-1006, 2000.

[25] P. Stumm and D. Drabold, “Can amorphous GaN serve as a useful electronic material?" Physical Review Letters, vol. 79, no. 4, p. 677, 1997.

[26] H. Hovel and J. Cuomo, "Electrical and optical properties of rf-sputtered GaN and InN," Applied Physics Letters, vol. 20, no. 2, pp. 71-73, 1972.

[27] J. Vesely, M. Shatzkes, and P. Burkhardt, "Space-charge-limited current flow in gallium nitride thin films," Physical Review B, vol. 10, no. 2, p. 582, 1974.

[28] S. Kobayashi, S. Nonomura, T. Ohmori, K. Abe, S. Hirata, T. Uno, T. Gotoh, and S. Nitta, “Optical and electrical properties of amorphous and microcrystalline $\mathrm{GaN}$ films and their application to transparent TFT," Applied Surface Science, vol. 113, pp. 480-484, 1997.

[29] S. Kobayashi, S. Nonomura, K. Ushikoshi, K. Abe, M. Nishio, H. Furukawa, T. Gotoh, and S. Nitta "Optical and electrical properties of nano-crystalline GaN thin films and their application for thin-film transistor," Journal of Crystal Growth, vol. 189, pp. 749-752, 1998.

[30] S. Yagi, S. Suzuki, and T. Iwanaga, "Hydrogenated polycrystalline GaN surface light-emitting devices on transparent conductive glass," Japanese Journal of Applied Physics, vol. 40, no. 12B, p. L1349, 2001.

[31] H. Tampo, T. Yamanaka, K. Yamada, K. Ohnishi, M. Hashimoto, and H. Asahi, "Field emission from polycrystalline GaN grown on Mo substrate," Japanese Journal of Applied Physics, vol. 41, no. 8B, p. L907, 2002.

[32] S. Hasegawa, S. Nishida, T. Yamashita, and H. Asahi, "Polycrystalline GaN for light emitter and field electron emitter applications," Thin Solid Films, vol. 487, no. 1-2, pp. 260-267, 2005.

[33] T. Yamashita, S. Hasegawa, S. Nishida, M. Ishimaru, Y. Hirotsu, and H. Asahi, "Electron field emission from $\mathrm{GaN}$ nanorod films grown on $\mathrm{Si}$ substrates with native silicon oxides," Applied Physics Letters, vol. 86, no. 8, p. 082109, 2005.

[34] V. Christie, S. Liem, R. Reeves, V. Kennedy, A. Markwitz, and S. Durbin, “Characterisation of polycrystalline gallium nitride grown by plasma-assisted evaporation," Current Applied Physics, vol. 4, no. 2-4, pp. 225-228, 2004.

[35] D. Ghosh, S. Hussain, B. Ghosh, R. Bhar, and A. Pal, "Stress and grain boundary properties of GaN films prepared by pulsed laser deposition technique," ISRN Materials Science, vol. 2014, 2014.

[36] N. Biyikli and A. Haider, "Atomic layer deposition: an enabling technology for the growth of functional nanoscale semiconductors," Semiconductor Science and Technology, vol. 32, no. 9, p. 093002, 2017.

[37] C. Ozgit-Akgun, E. Goldenberg, A. K. Okyay, and N. Biyikli, “Hollow cathode plasma-assisted atomic layer deposition of crystalline $\mathrm{AlN}, \mathrm{GaN}$ and $\mathrm{Al} \times \mathrm{Ga} 1-\mathrm{x} \mathrm{N}$ thin films at low temperatures," Journal of Materials Chemistry C, vol. 2, no. 12, pp. 2123-2136, 2014.

[38] N. Karam, T. Parodos, P. Colter, D. McNulty, W. Rowland, J. Schetzina, N. El-Masry, and S. M. Bedair, "Growth of device quality GaN at $550 \mathrm{C}$ by atomic layer epitaxy," Applied Physics Letters, vol. 67, no. 1, pp. 94-96, 1995.

[39] S. Bolat, C. Ozgit-Akgun, B. Tekcan, N. Biyikli, and A. Okyay, "Low temperature thin film transistors with hollow cathode plasma-assisted atomic layer deposition based GaN channels," Applied Physics Letters, vol. 104, no. 24, p. 243505, 2014.

[40] S. Bolat, B. Tekcan, C. Ozgit-Akgun, N. Biyikli, and A. K. Okyay, "Electronic and optical device applications of hollow cathode plasma assisted atomic layer deposition based gan thin films," Journal of Vacuum Science \& Technology A: Vacuum, Surfaces, and Films, vol. 33, no. 1, p. 01A143, 2015.

[41] S. Bolat, Z. Sisman, and A. Okyay, "Demonstration of flexible thin film transistors with GaN channels," Applied Physics Letters, vol. 109, no. 23, p. 233504, 2016.

[42] G. Gupta, S. Banerjee, S. Dutta, A. A. Aarnink, J. Schmitz, A. Y. Kovalgin, and R. J. E. Hueting, “Charge carrier transport and electroluminescence in atomic layer deposited poly-GaN/c-Si heterojunction diodes," Journal of Applied Physics, vol. 124, no. 8, p. 084503, 2018.

[43] O. Ambacher, B. Foutz, J. Smart, J. R. Shealy, N. G. Weimann, K. Chu, M. Murphy, A. J. Sierakowski, W. J. Schaff, L. F. Eastman, R. Dimitrov, A. Mitchell, and M. Stutzmann, “Two dimensional electron gases induced by spontaneous and piezoelectric polarization in undoped and doped AlGaN/GaN heterostructures," Journal of Applied Physics, vol. 87, no. 1, pp. 334-344, 2000.

[44] D. Jena, J. Simon, A. K. Wang, Y. Cao, K. Goodman, J. Verma, S. Ganguly, G. Li, K. Karda, V. Protasenko et al., "Polarization-engineering in group III-nitride heterostructures: New opportunities for device design," Physica Status Solidi (a), vol. 208, no. 7, pp. 1511-1516, 2011.

[45] W. H. Brattain and J. Bardeen, "Nature of the forward current in Germanium point contacts," Phys. Rev., vol. 74, pp. 231-232, Jul. 1948. 
[46] G. Gupta, B. Rajasekharan, and R. J. E. Hueting, "Electrostatic Doping in Semiconductor Devices," IEEE Transactions on Electron Devices, vol. 64, no. 8, pp. 3044-3055, Aug. 2017.

[47] F. Riederer, T. Grap, S. Fischer, M. R. Mueller, D. Yamaoka, B. Sun, C. Gupta, K. T. Kallis, and J. Knoch, "Alternatives for Doping in Nanoscale Field-Effect Transistors," Physica Status Solidi (a), vol. 215, no. 7, p. 1700969, 2018.

[48] S. Cristoloveanu, K. H. Lee, H. Park, and M. S. Parihar, "The concept of electrostatic doping and related devices," Solid-State Electronics, vol. 155, pp. 32-43, 2019.

[49] K. T. Chern, L. J. Guido, T. A. Ciarkowski, N. P. Allen, O. A. Laboutin, R. E. Welser, and V. C. Elarde, "GaInN/GaN-Ni/Au transparent conducting oxide Schottky barrier solar cells," in 2014 IEEE 40th Photovoltaic Specialist Conference (PVSC), 2014, pp. 1139-1142.

[50] K. T. Chern, N. P. Allen, T. A. Ciarkowski, O. A. Laboutin, R. E. Welser, and L. J. Guido, “GaInN/GaN solar cells made without p-type material using oxidized Ni/Au Schottky electrodes," Materials Science in Semiconductor Processing, vol. 55, pp. 2-6, 2016.

[51] H.-B. Lee, H.-I. Cho, H.-S. An, Y.-H. Bae, M.-B. Lee, J.-H. Lee, and S.-H. Hahm, “A normally off GaN nMOSFET with Schottky-barrier source and drain on a Si-auto-doped p-GaN/Si," IEEE Electron Device Letters, vol. 27, no. 2, pp. 81-83, 2006.

[52] S. A. Loan, S. Verma, and A. M. Alamoud, "High-performance charge plasma based normally OFF GaN MOSFET," Electronics Letters, vol. 52, no. 8, pp. 656-658, 2016.

[53] X. Liu, C. Shan, H. Zhu, B. Li, M. Jiang, S. Yu, and D. Shen, “Ultraviolet lasers realized via electrostatic doping method," Scientific Reports, vol. 5, p. 13641, 2015.

[54] W. Bradley, "The surface-barrier transistor: Part I-Principles of the surface-barrier transistor," Proceedings of the IRE, vol. 41, no. 12, pp. 1702-1706, 1953.

[55] M. Green and J. Shewchun, "Minority carrier effects upon the small signal and steady-state properties of Schottky diodes," Solid-State Electronics, vol. 16, no. 10, pp. 1141-1150, 1973.

[56] G. Gupta, S. Dutta, S. Banerjee, and R. J. E. Hueting, "Minority Carrier Injection in High-Barrier SiSchottky Diodes," IEEE Transactions on Electron Devices, vol. 65, no. 4, pp. 1276-1282, Apr. 2018.

[57] G. Gupta, F. Mema, and R. J. E. Hueting, “Electron-hole bilayer light-emitting device: concept and operation," Solid-State Electronics, vol. 168, p. 107726, June 2020.

[58] M. Lepselter and S. Sze, "SB-IGFET: An insulated-gate field-effect transistor using Schottky barrier contacts for source and drain," in Proc. IEEE, vol. 56, no. 8, Aug 1968, pp. 1400-1402.

[59] J. M. Larson and J. P. Snyder, "Overview and status of metal S/D Schottky-barrier MOSFET technology," IEEE Transactions on Electron Devices, vol. 53, no. 5, pp. 1048-1058, May 2006.

[60] R. J. E. Hueting, B. Rajasekharan, C. Salm, and J. Schmitz, “The charge plasma p-n diode," IEEE Electron Device Letters, vol. 29, no. 12, pp. 1367-1369, Dec 2008.

[61] B. Rajasekharan, R. J. E. Hueting, C. Salm, T. van Hemert, R. A. M. Wolters, and J. Schmitz, “Fabrication and characterization of the charge-plasma diode," IEEE Electron Device Letters, vol. 31, no. 6, pp. 528530, June 2010.

[62] M. J. Kumar and K. Nadda, "Bipolar charge-plasma transistor: A novel three terminal device," IEEE Transactions on Electron Devices, vol. 59, no. 4, pp. 962-967, April 2012.

[63] M. J. Kumar and S. Janardhanan, "Doping-less tunnel field effect transistor: Design and investigation," IEEE Transactions on Electron Devices, vol. 60, no. 10, pp. 3285-3290, Oct 2013.

[64] M. D. Marchi, D. Sacchetto, S. Frache, J. Zhang, P. E. Gaillardon, Y. Leblebici, and G. D. Micheli, "Polarity control in double-gate, gate-all-around vertically stacked silicon nanowire FETs," in Proceedings of the International Electron Devices Meeting (IEDM 2012), Dec 2012, pp. 8.4.1-8.4.4.

[65] A. Heinzig, S. Slesazeck, F. Kreupl, T. Mikolajick, and W. M. Weber, “Reconfigurable silicon nanowire transistors," Nano Letters, vol. 12, no. 1, pp. 119-124, 2012.

[66] F. Wessely, T. Krauss, and U. Schwalke, “CMOS without doping: Multi-gate silicon-nanowire fieldeffect-transistors," Solid-State Electronics, vol. 70, pp. 33-38, 2012

[67] K. Lee, M. Bawedin, M. Parihar, H.-J. Park, and S. Cristoloveanu, “The Virtual Diode with Electrostatic Doping," ECS Transactions, vol. 77, no. 5, pp. 191-196, 2017.

[68] S. Cristoloveanu, K. H. Lee, and M. Bawedin, "A reconfigurable silicon-on-insulator diode with tunable electrostatic doping," Journal of Applied Physics, vol. 122, no. 8, p. 084502, 2017.

[69] K. H. Lee and S. Cristoloveanu, "Esaki Diode in Undoped Silicon Film," IEEE Electron Device Letters, vol. 40, no. 9, pp. 1346-1349, 2019.

[70] K. S. Novoselov, A. K. Geim, S. V. Morozov, D. Jiang, Y. Zhang, S. V. Dubonos, I. V. Grigorieva, and A. A. Firsov, "Electric field effect in atomically thin carbon films," Science, vol. 306, no. 5696, pp. 666669, 2004.

[71] J. R. Williams, L. DiCarlo, and C. M. Marcus, “Quantum hall effect in a gate-controlled p-n junction of graphene," Science, vol. 317, no. 5838, pp. 638-641, 2007. 
[72] G. Giovannetti, P. A. Khomyakov, G. Brocks, V. M. Karpan, J. van den Brink, and P. J. Kelly, “Doping graphene with metal contacts," Physical Review Letters, vol. 101, p. 026803, Jul 2008.

[73] J. F. Tian, L. A. Jauregui, G. Lopez, H. Cao, and Y. P. Chen, “Ambipolar graphene field effect transistors by local metal side gates," Applied Physics Letters, vol. 96, no. 26, 2010.

[74] J. U. Lee, P. P. Gipp, and C. M. Heller, “Carbon nanotube p-n junction diodes," Applied Physics Letters, vol. 85, no. 1, pp. 145-147, 2004.

[75] S. Heinze, J. Tersoff, R. Martel, V. Derycke, J. Appenzeller, and P. Avouris, “Carbon nanotubes as Schottky barrier transistors," Physical Review Letters, vol. 89, p. 106801, Aug 2002.

[76] J. Appenzeller, J. Knoch, V. Derycke, R. Martel, S. Wind, and P. Avouris, "Field-modulated carrier transport in carbon nanotube transistors," Physical Review Letters, vol. 89, p. 126801, Aug 2002.

[77] H. Ilatikhameneh, G. Klimeck, J. Appenzeller, and R. Rahman, "Scaling theory of electrically doped 2D transistors," IEEE Electron Device Letters, vol. 36, no. 7, pp. 726-728, July 2015.

[78] H. Ilatikhameneh, T. A. Ameen, G. Klimeck, J. Appenzeller, and R. Rahman, “Dielectric engineered tunnel field-effect transistor," IEEE Electron Device Letters, vol. 36, no. 10, pp. 1097-1100, Oct 2015.

[79] L. Lattanzio, L. D. Michielis, and A. M. Ionescu, "The electron-hole bilayer tunnel FET," Solid-State Electronics, vol. 74, pp. $85-90,2012$.

[80] _ _Electron-hole bilayer tunnel FET for steep subthreshold swing and improved ON current," in Proc. ESSDERC, Sept 2011, pp. 259-262.

[81] A. Revelant, A. Villalon, Y. Wu, A. Zaslavsky, C. L. Royer, H. Iwai, and S. Cristoloveanu, “Electronhole bilayer TFET: Experiments and comments," IEEE Transactions on Electron Devices, vol. 61, no. 8, pp. 2674-2681, Aug 2014.

[82] G. Gupta, F. Mema, and R. J. E. Hueting, "InAs Electron-Hole Bilayer LED," in Joint International EUROSOI Workshop and International Conference on Ultimate Integration on Silicon (EUROSOI-ULIS). IEEE, 2019.

[83] E. H. Rhoderick and R. H. Williams, Metal-Semiconductor Contacts. Clarendon Press Oxford, 1988, vol. 129.

[84] Y.Nishi, "Insulated gate field effect transistor and its manufacturing method," Japanese Patent 587527 , Patent 587 527, 1970.

[85] S.-M. Koo, M. D. Edelstein, Q. Li, C. A. Richter, and E. M. Vogel, "Silicon nanowires as enhancementmode Schottky barrier field-effect transistors," Nanotechnology, vol. 16, no. 9, p. 1482, 2005.

[86] J. Zhang, M. De Marchi, P.-E. Gaillardon, and G. De Micheli, “A Schottky-barrier silicon FinFET with $6.0 \mathrm{mv} / \mathrm{dec}$ subthreshold slope over 5 decades of current," in Proceedings of the International Electron Devices Meeting (IEDM 2014), 2014.

[87] Y.-M. Lin, J. Appenzeller, J. Knoch, and P. Avouris, “High-performance carbon nanotube field-effect transistor with tunable polarities," IEEE Transactions on Nanotechnology, vol. 4, no. 5, pp. 481-489, Sept 2005.

[88] F. Wessely, T. Krauss, and U. Schwalke, "Virtually dopant-free CMOS: Midgap schottky-barrier nanowire field-effect-transistors for high temperature applications," Solid-State Electronics, vol. 74, pp. $91-96,2012$.

[89] J. A. Misewich, R. Martel, P. Avouris, J. C. Tsang, S. Heinze, and J. Tersoff, “Electrically induced optical emission from a carbon nanotube FET," Science, vol. 300, no. 5620, pp. 783-786, 2003.

[90] W. A. Anderson and A. E. Delahoy, "Schottky barrier diodes for solar energy conversion," Proceedings of the IEEE, vol. 60, no. 11, pp. 1457-1458, Nov 1972.

[91] L. Zhang, Y. Jia, S. Wang, Z. Li, C. Ji, J. Wei, H. Zhu, K. Wang, D. Wu, E. Shi et al., “Carbon nanotube and CdSe nanobelt Schottky junction solar cells," Nano letters, vol. 10, no. 9, pp. 3583-3589, 2010.

[92] Y. Ye, Y. Dai, L. Dai, Z. Shi, N. Liu, F. Wang, L. Fu, R. Peng, X. Wen, Z. Chen et al., "High-performance single CdS nanowire (nanobelt) Schottky junction solar cells with Au/graphene Schottky electrodes," ACS applied materials $\mathcal{E}$ interfaces, vol. 2, no. 12, pp. 3406-3410, 2010.

[93] M. Shanmugam, C. A. Durcan, and B. Yu, "Layered semiconductor molybdenum disulfide nanomembrane based Schottky-barrier solar cells," Nanoscale, vol. 4, pp. 7399-7405, 2012.

[94] E. Demoulin and F. Van De Wiele, "Inversion layer at the interface of Schottky diodes," Solid-State Electronics, vol. 17, no. 8, pp. 825-833, 1974.

[95] K. Nadda and M. J. Kumar, "Vertical bipolar charge plasma transistor with buried metal layer," Scientific Reports, vol. 5, p. 7860, Jan. 2015.

[96] M. J. Kumar and S. Sharma, "GaAs tunnel diode with electrostatically doped n-region: Proposal and analysis," IEEE Transactions on Electron Devices, vol. 62, no. 10, pp. 3445-3448, Oct. 2015.

[97] J. Colinge, “Multi-gate SOI MOSFETs," Microelectronic Engineering, vol. 84, no. 9, pp. 2071-2076, 2007.

[98] Sentaurus TCAD, Version 1-2016.03 ed., Synopsys Inc., Mountain View, CA, USA, 2016.

[99] Y. Taur, X. Liang, W. Wang, and H. Lu, "A continuous, analytic drain-current model for DG MOSFETs," 
IEEE Electron Device Letters, vol. 25, no. 2, pp. 107-109, Feb 2004

[100] T. van Hemert, R. J. E. Hueting, B. Rajasekharan, C. Salm, and J. Schmitz, “On the modelling and optimisation of a novel Schottky based silicon rectifier," in 2010 Proceedings of the European Solid State Device Research Conference. IEEE, 2010, pp. 460-463.

[101] M. Bokdam, P. A. Khomyakov, G. Brocks, Z. Zhong, and P. J. Kelly, “Electrostatic doping of graphene through ultrathin hexagonal boron nitride films," Nano Letters, vol. 11, no. 11, pp. 4631-4635, 2011, pMID: 21936569.

[102] J. Knoch, Z. Chen, and J. Appenzeller, "Properties of metal-graphene contacts," IEEE Transactions on Nanotechnology, vol. 11, no. 3, pp. 513-519, 2012.

[103] I. Meric, M. Y. Han, A. F. Young, B. Ozyilmaz, P. Kim, and K. L. Shepard, “Current saturation in zerobandgap, top-gated graphene field-effect transistors," Nature nanotechnology, vol. 3, no. 11, pp. 654-659, 2008.

[104] J. Appenzeller, Y.-M. Lin, J. Knoch, and P. Avouris, "Band-to-band tunneling in carbon nanotube fieldeffect transistors," Physical review letters, vol. 93, no. 19, p. 196805, 2004.

[105] M. Prunnila and J. Ahopelto, "Two sub-band conductivity of Si quantum well," Physica E: Lowdimensional Systems and Nanostructures, vol. 32, no. 1-2, pp. 281-284, 2006

[106] M. Prunnila, S. J. Laakso, J. M. Kivioja, and J. Ahopelto, “Electrons and holes in Si quantum well: A room-temperature transport and drag resistance study," Applied Physics Letters, vol. 93, no. 11, 2008.

[107] H.-K. Lim and J. G. Fossum, "Threshold voltage of thin-film silicon-on-insulator (SOI) MOSFET's," IEEE Transactions on Electron Devices, vol. 30, no. 10, pp. 1244-1251, Oct 1983.

[108] C. Alper, L. Lattanzio, L. D. Michielis, P. Palestri, L. Selmi, and A. M. Ionescu, “Quantum mechanical study of the germanium electron-hole bilayer tunnel FET," IEEE Transactions on Electron Devices, vol. 60, no. 9, pp. 2754-2760, Sept 2013.

[109] M. Ancona and H. Tiersten, "Macroscopic physics of the silicon inversion layer," Physical Review B, vol. 35 , no. 15 , p. $7959,1987$.

[110] S. Cristoloveanu, J. Wan, and A. Zaslavsky, "A Review of sharp-switching devices for ultra-low power applications," IEEE Journal of the Electron Devices Society, vol. 4, no. 5, pp. 215-226, Sept 2016.

[111] K. Nadda and M. J. Kumar, "Schottky collector bipolar transistor without impurity doped emitter and base: Design and performance," IEEE Transactions on Electron Devices, vol. 60, no. 9, pp. 2956-2959, Sept 2013.

[112] C. Sahu, A. Ganguly, and J. Singh, "Design and performance projection of symmetric bipolar chargeplasma transistor on SOI," Electronics Letters, vol. 50, no. 20, pp. 1461-1463, Sept 2014.

[113] S. Singh and P. N. Kondekar, “Dopingless super-steep impact ionisation MOS (dopingless-IMOS) based on workfunction engineering," Electronics Letters, vol. 50, no. 12, pp. 888-889, June 2014.

[114] C. Sahu and J. Singh, "Charge-plasma based process variation immune junctionless transistor," IEEE Electron Device Letters, vol. 35, no. 3, pp. 411-413, March 2014.

[115] M. J. Kumar, M. Maheedhar, and P. P. Varma, "Junctionless biristor: A bistable resistor without chemically doped p-n junctions," IEEE Journal of the Electron Devices Society, vol. 3, no. 4, pp. 311-315, July 2015.

[116] K. Pradhan, P. Sahu, D. Singh, L. Artola, and S. Mohapatra, "Reliability analysis of charge plasma based double material gate oxide (DMGO) sige-on-insulator (SGOI) MOSFET," Superlattices and Microstructures, vol. 85, pp. 149-155, 2015.

[117] C. Shan, Y. Wang, and M. T. Bao, "A charge-plasma-based transistor with induced graded channel for enhanced analog performance," IEEE Transactions on Electron Devices, vol. 63, no. 6, pp. 2275-2281, June 2016.

[118] A. Pan and C. O. Chui, “Gate-induced source tunneling FET (GISTFET)," IEEE Transactions on Electron Devices, vol. 62, no. 8, pp. 2390-2395, Aug 2015.

[119] J. A. Seamons, D. R. Tibbetts, J. L. Reno, and M. P. Lilly, “Undoped electron-hole bilayers in a GaAs/AlGaAs double quantum well," Applied Physics Letters, vol. 90, no. 5, 2007.

[120] K. Nadda and M. J. Kumar, “Thin-film bipolar transistors on recrystallized polycrystalline silicon without impurity doped junctions: Proposal and investigation," Journal of Display Technology, vol. 10, no. 7, pp. 590-594, Jul 2014.

[121] C. C. Hsu, H. P. Chen, and W. C. Ting, “Correlation between carrier concentration distribution, I-V and C-V characteristics of a-InGaZnO TFTs," Journal of Display Technology, vol. 12, no. 4, pp. 328-337, April 2016.

[122] H. Liu, A. T. Neal, and P. D. Ye, “Channel length scaling of MoS2 MOSFETs," ACS Nano, vol. 6, no. 10, pp. 8563-8569, 2012, pMID: 22957650.

[123] S. Chuang, C. Battaglia, A. Azcatl, S. McDonnell, J. S. Kang, X. Yin, M. Tosun, R. Kapadia, H. Fang, R. M. Wallace et al., "MoS2 p-type transistors and diodes enabled by high work function MoOx contacts," Nano Letters, vol. 14, no. 3, pp. 1337-1342, 2014. 
[124] H. Ilatikhameneh, Y. Tan, B. Novakovic, G. Klimeck, R. Rahman, and J. Appenzeller, "Tunnel fieldeffect transistors in 2-D transition metal dichalcogenide materials," IEEE Journal on Exploratory SolidState Computational Devices and Circuits, vol. 1, pp. 12-18, 2015.

[125] H. B. Michaelson, "The work function of the elements and its periodicity," Journal of Applied Physics, vol. 48, no. 11, pp. 4729-4733, 1977.

[126] C. Battaglia, X. Yin, M. Zheng, I. D. Sharp, T. Chen, S. McDonnell, A. Azcatl, C. Carraro, B. Ma, R. Maboudian et al., "Hole selective MoOx contact for silicon solar cells," Nano Letters, vol. 14, no. 2, pp. 967-971, 2014.

[127] N. Mott, "Note on the contact between a metal and an insulator or semi-conductor," in Mathematical Proceedings of the Cambridge Philosophical Society, vol. 34, no. 04. Cambridge Univ Press, 1938, pp. 568-572.

[128] R. T. Tung, "The physics and chemistry of the Schottky barrier height," Applied Physics Reviews, vol. 1, no. 1, 2014.

[129] R. Lieten, V. Afanas'ev, N. Thoan, S. Degroote, W. Walukiewicz, and G. Borghs, "Mechanisms of Schottky barrier control on n-type germanium using Ge3N4 interlayers," Journal of The Electrochemical Society, vol. 158, no. 4, pp. H358-H362, 2011.

[130] K. H. Kao and L. Y. Chen, “A Dopingless FET with Metal-Insulator-Semiconductor Contacts," IEEE Electron Device Letters, vol. 38, no. 1, pp. 5-8, Jan. 2017.

[131] Y. Liu, J. Guo, E. Zhu, L. Liao, S.-J. Lee, M. Ding, I. Shakir, V. Gambin, Y. Huang, and X. Duan, “Approaching the Schottky-Mott limit in van der Waals metal-semiconductor junctions," Nature, vol. 557, no. 7707, p. $696,2018$.

[132] H. F. Dadgour, K. Endo, V. K. De, and K. Banerjee, “Grain-orientation induced work function variation in nanoscale metal-gate transistors-Part I: Modeling, analysis, and experimental validation," IEEE Transactions on Electron Devices, vol. 57, no. 10, pp. 2504-2514, Oct 2010.

[133] _ _ "Grain-orientation induced work function variation in nanoscale metal-gate transistors-Part II: Implications for process, device, and circuit design," IEEE Transactions on Electron Devices, vol. 57, no. 10 , pp. 2515-2525, Oct 2010

[134] T. Matsukawa, Y. Liu, K. Endo, J. Tsukada, H. Yamauchi, Y. Ishikawa, S. O'uchi, W. Mizubayashi, H. Ota, S. Migita et al., "Influence of work function variation of metal gates on fluctuation of subthreshold drain current for fin field-effect transistors with undoped channels," Japanese Journal of Applied Physics, vol. 53, no. 4S, p. 04EC11, 2014.

[135] E. Rhoderick, "Comments on the conduction mechanism in Schottky diodes," Journal of Physics D: Applied Physics, vol. 5, no. 10, p. 1920, Oct. 1972.

[136] R. Schwarz and J. Walsh, "Part V-The Properties of Metal to Semiconductor Contacts," Proceedings of the IRE, vol. 41, no. 12, pp. 1715-1720, 1953.

[137] D. Scharfetter, "Minority carrier injection and charge storage in epitaxial Schottky barrier diodes," Solid-State Electronics, vol. 8, no. 3, pp. 299-311, 1965.

[138] A. Yu and E. Snow, "Minority carrier injection of metal-silicon contacts," Solid-State Electronics, vol. 12, no. 3, pp. 155-160, 1969.

[139] M. Alavi, D. K. Reinhard, and C. Yu, "Minority-carrier injection in Pt-Si Schottky-barrier diodes at high current densities," IEEE Transactions on Electron Devices, vol. 34, no. 5, pp. 1134-1140, 1987.

[140] T. T. Mnatsakanov, M. E. Levinshtein, A. G. Tandoev, S. N. Yurkov, and J. W. Palmour, "Minority carrier injection and current-voltage characteristics of Schottky diodes at high injection level," SolidState Electronics, vol. 121, pp. 41-46, 2016.

[141] C. Crowell and S. Sze, "Current transport in metal-semiconductor barriers," Solid-State Electronics, vol. 9, no. 11-12, pp. 1035-1048, Nov./Dec/ 1966.

[142] B. Elfsten and P. Tove, "Calculation of charge distributions and minority-carrier injection ratio for highbarrier Schottky diodes," Solid-State Electronics, vol. 28, no. 7, pp. 721-727, 1985.

[143] Y. Amemiya and Y. Mizushima, "Bipolar-mode Schottky contact and applications to high-speed diodes," IEEE Transactions on Electron Devices, vol. 31, no. 1, pp. 35-42, 1984.

[144] K. Nill, A. Calawa, T. Harman, and J. Walpole, "Laser emission from metal-semiconductor barriers on PbTe and Pb0.8Sn0.2Te," Applied Physics Letters, vol. 16, no. 10, pp. 375-377, 1970.

[145] D. B. M. Klaassen, "A unified mobility model for device simulation-I. Model equations and concentration dependence," Solid-State Electronics, vol. 35, no. 7, pp. 953-959, Jul. 1992.

[146] B. J. Baliga, "Analysis of a high-voltage merged p-i-n/Schottky (MPS) rectifier," IEEE Electron Device Letters, vol. 8, no. 9, pp. 407-409, Sep. 1987.

[147] H. Gummel and H. Poon, "An integral charge control model of bipolar transistors," Bell System Technical Journal, vol. 49, no. 5, pp. 827-852, May/Jun. 1970.

[148] M. Wittmer, "Current transport in high-barrier IrSi/Si Schottky diodes," Physical Review B, vol. 42, pp. 5249-5259, Sep. 1990. 
[149] _ "Conduction mechanism in PtSi/Si Schottky diodes," Physical Review B, vol. 43, no. 5, p. 4385, Feb. 1991.

[150] J. Werner, A. Levi, R. Tung, M. Anzlowar, and M. Pinto, “Origin of the excess capacitance at intimate Schottky contacts," Physical Review Letters, vol. 60, no. 1, p. 53, 1988.

[151] D. Connelly, C. Faulkner, D. Grupp, and J. Harris, "A new route to zero-barrier metal source/drain MOSFETs," IEEE Transactions on Nanotechnology, vol. 3, no. 1, pp. 98-104, Mar. 2004.

[152] J. Andrews and M. Lepselter, "Reverse current-voltage characteristics of metal-silicide Schottky diodes," Solid-State Electronics, vol. 13, no. 7, pp. 1011-1023, Jul. 1970.

[153] P. McCafferty, A. Sellai, P. Dawson, and H. Elabd, “Barrier characteristics of PtSi/p-Si Schottky diodes as determined from I-V-T measurements," Solid-State Electronics, vol. 39, no. 4, pp. 583-592, Apr. 1996.

[154] L. Qi, G. Lorito, and L. K. Nanver, "Lateral-transistor test structures for evaluating the effectiveness of surface doping techniques," IEEE Transactions on Semiconductor Manufacturing, vol. 25, no. 4, pp. 581-588, Nov. 2012.

[155] X. Liu and L. K. Nanver, "Comparing current flows in ultrashallow pn-/Schottky-like diodes with 2diode test method," in Microelectronic Test Structures (ICMTS), 2016 International Conference on. IEEE, 2016, pp. 190-195.

[156] K. Mok, L. Qi, A. Vlooswijk, and L. K. Nanver, "Self-aligned two-layer metallization with low series resistance for litho-less contacting of large-area photodiodes," Solid-State Electronics, vol. 111, pp. 210 217,2015 .

[157] E. F. Schubert, T. Gessmann, and J. K. Kim, Light Emitting Diodes. Wiley Online Library, Jul. 2005.

[158] V. Puliyankot and R. J. E. Hueting, “One-dimensional physical model to predict the internal quantum efficiency of Si-based LEDs," IEEE Transactions on Electron Devices, vol. 59, no. 1, pp. 26-34, Jan. 2012.

[159] D. A. B. Miller, "Device requirements for optical interconnects to silicon chips," Proceedings of the IEEE, vol. 97, no. 7, pp. 1166-1185, Jul. 2009.

[160] Y. Wu, T. Hasan, X. Li, P. Xu, Y. Wang, X. Shen, X. Liu, and Q. Yang, "High efficiency single Ag nanowire/p-GaN substrate Schottky junction-based ultraviolet light emitting diodes," Applied Physics Letters, vol. 106, no. 5, p. 051108, 2015.

[161] J. Meyer, S. Hamwi, M. Kröger, W. Kowalsky, T. Riedl, and A. Kahn, “Transition metal oxides for organic electronics: energetics, device physics and applications," Advanced Materials, vol. 24, no. 40, pp. 5408-5427, 2012.

[162] L. G. Gerling, S. Mahato, A. Morales-Vilches, G. Masmitja, P. Ortega, C. Voz, R. Alcubilla, and J. Puigdollers, "Transition metal oxides as hole-selective contacts in silicon heterojunctions solar cells," Solar Energy Materials and Solar Cells, vol. 145, pp. 109-115, 2016.

[163] J. Bullock, P. Zheng, Q. Jeangros, M. Tosun, M. Hettick, C. M. Sutter-Fella, Y. Wan, T. Allen, D. Yan, D. Macdonald et al., "Lithium Fluoride Based Electron Contacts for High Efficiency n-Type Crystalline Silicon Solar Cells," Advanced Energy Materials, vol. 6, no. 14, p. 1600241, 2016.

[164] C. Waldauf, M. Morana, P. Denk, P. Schilinsky, K. Coakley, S. Choulis, and C. Brabec, "Highly efficient inverted organic photovoltaics using solution based titanium oxide as electron selective contact," Applied Physics Letters, vol. 89, no. 23, p. 233517, 2006.

[165] X. Yang, P. Zheng, Q. Bi, and K. Weber, "Silicon heterojunction solar cells with electron selective TiOx contact," Solar Energy Materials and Solar Cells, vol. 150, pp. 32-38, 2016.

[166] M. Green, F. King, and J. Shewchun, "Minority carrier MIS tunnel diodes and their application to electron-and photo-voltaic energy conversion-I. Theory," Solid-State Electronics, vol. 17, no. 6, pp. 551561,1974

[167] J. Shewchun, M. Green, and F. King, "Minority carrier MIS tunnel diodes and their application to electron-and photo-voltaic energy conversion-II. Experiment," Solid-State Electronics, vol. 17, no. 6, pp. 563-572, 1974.

[168] D. Chi, R. Lee, S. Chua, S. Lee, S. Ashok, and D.-L. Kwong, “Current-voltage characteristics of Schottky barriers with barrier heights larger than the semiconductor band gap: The case of $\mathrm{Ni} \mathrm{Ge/n-(001)} \mathrm{Ge}$ contact," Journal of Applied Physics, vol. 97, no. 11, p. 113706, 2005.

[169] G. Gupta, S. D. Thammaiah, R. J. E. Hueting, and L. K. Nanver, "In search of a hole inversion layer in $\mathrm{Pd} / \mathrm{MoOx} / \mathrm{Si}$ diodes through I-V characterization using dedicated ring-shaped test structures," in 2019 IEEE 32nd International Conference on Microelectronic Test Structures (ICMTS), 2019, pp. 12-17.

[170] M. A. Juntunen, J. Heinonen, H. S. Laine, V. Vähänissi, P. Repo, A. Vaskuri, and H. Savin, “N-type induced junction black silicon photodiode for UV detection," in Integrated Photonics: Materials, Devices, and Applications IV, vol. 10249. International Society for Optics and Photonics, 2017, p. 102490I.

[171] T. Knežević, X. Liu, E. Hardeveld, T. Suligoj, and L. K. Nanver, “Limits on Thinning of Boron Layers With/Without Metal Contacting in PureB Si (Photo) Diodes," IEEE Electron Device Letters, vol. 40, no. 6 , pp. 858-861, 2019.

[172] L. K. Nanver, L. Qi, V. Mohammadi, K. Mok, W. B. de Boer, N. Golshani, A. Sammak, T. L. Scholtes, 
A. Gottwald, U. Kroth et al., "Robust UV/VUV/EUV PureB photodiode detector technology with high CMOS compatibility," IEEE Journal of Selected Topics in Quantum Electronics, vol. 20, no. 6, pp. 306-316, 2014.

[173] C. Battaglia, S. M. De Nicolas, S. De Wolf, X. Yin, M. Zheng, C. Ballif, and A. Javey, "Silicon heterojunction solar cell with passivated hole selective MoOx contact," Applied Physics Letters, vol. 104, no. 11, p. 113902, 2014.

[174] J. Bullock, A. Cuevas, T. Allen, and C. Battaglia, “Molybdenum oxide MoOx: A versatile hole contact for silicon solar cells," Applied Physics Letters, vol. 105, no. 23, p. 232109, 2014.

[175] M. Gao, D. Chen, B. Han, W. Song, M. Zhou, X. Song, F. Xu, L. Zhao, Y. Li, and Z. Ma, “Bifunctional Hybrid a-SiOx(Mo) Layer for Hole-Selective and Interface Passivation of Highly Efficient MoOx/a$\mathrm{SiOx}(\mathrm{Mo}) / \mathrm{n}-\mathrm{Si}$ Heterojunction Photovoltaic Device," ACS Applied Materials E Interfaces, vol. 10, no. 32, pp. $27454-27464,2018$.

[176] R. T. Tung, "Chemical bonding and Fermi level pinning at metal-semiconductor interfaces," Physical Review Letters, vol. 84, no. 26, p. 6078, 2000.

[177] T. Sun, R. Wang, R. Liu, C. Wu, Y. Zhong, Y. Liu, Y. Wang, Y. Han, Z. Xia, Y. Zou et al., “Investigation of MoOx/n-Si strong inversion layer interfaces via dopant-free heterocontact," Physica Status Solidi (RRL)-Rapid Research Letters, vol. 11, no. 7, p. 1700107, 2017.

[178] F. Balestra, S. Cristoloveanu, M. Benachir, J. Brini, and T. Elewa, “Double-gate silicon-on-insulator transistor with volume inversion: A new device with greatly enhanced performance," IEEE Electron Device Letters, vol. 8, no. 9, pp. 410-412, 1987.

[179] V. Mohammadi, S. Ramesh, and L. Nanver, "Thickness evaluation of deposited PureB layers in micro/millimeter-sized windows to Si," in International Conference on Microelectronic Test Structures (ICMTS). IEEE, 2014, pp. 194-199.

[180] L. G. Gerling, C. Voz, R. Alcubilla, and J. Puigdollers, “Origin of passivation in hole-selective transition metal oxides for crystalline silicon heterojunction solar cells," Journal of Materials Research, vol. 32, no. 2, pp. 260-268, 2017.

[181] A. Stephens, A. Aberle, and M. Green, "Surface recombination velocity measurements at the siliconsilicon dioxide interface by microwave-detected photoconductance decay," Journal of Applied Physics, vol. 76, no. 1, pp. 363-370, 1994.

[182] C. Messmer, M. Bivour, J. Schön, S. W. Glunz, and M. Hermle, “Numerical simulation of silicon heterojunction solar cells featuring metal oxides as carrier-selective contacts," IEEE Journal of Photovoltaics, vol. 8, no. 2, pp. 456-464, 2018.

[183] M. A. Green, "The capacitance of large barrier Schottky diodes," Solid State Electronics, vol. 19, pp. 421-422, 1976.

[184] H. Gummel and D. Scharfetter, "Depletion-Layer Capacitance of p+ n Step Junctions," Journal of Applied Physics, vol. 38, no. 5, pp. 2148-2153, 1967.

[185] T. Kamioka, Y. Hayashi, Y. Isogai, K. Nakamura, and Y. Ohshita, "Effects of annealing temperature on workfunction of $\mathrm{MoOx}$ at $\mathrm{MoOx} / \mathrm{SiO} 2$ interface and process-induced damage in indium tin oxide/MoOx/SiOx/Si stack," Japanese Journal of Applied Physics, vol. 57, no. 7, p. 076501, 2018.

[186] J. Fossum and M. Shibib, "A minority-carrier transport model for polysilicon contacts to silicon bipolar devices, including solar cells," in 1980 International Electron Devices Meeting. IEEE, 1980, pp. 280-283.

[187] L. Qi and L. K. Nanver, "Conductance along the interface formed by 400 deg C pure boron deposition on silicon," IEEE Electron Device Letters, vol. 36, no. 2, pp. 102-104, 2015.

[188] A. Boogaard, A. Y. Kovalgin, and R. Wolters, “Negative Charge in Plasma Oxidized SiO2 Layers," ECS Transactions, vol. 35, no. 4, p. 259, 2011

[189] G. Dingemans and W. Kessels, "Status and prospects of Al2O3-based surface passivation schemes for silicon solar cells," Journal of Vacuum Science E Technology A: Vacuum, Surfaces, and Films, vol. 30, no. 4, p. 040802, 2012.

[190] R. García-Hernansanz, E. Garcia-Hemme, D. Montero, J. Olea, A. del Prado, I. Martil, C. Voz, L. Gerling, J. Puigdollers, and R. Alcubilla, "Transport mechanisms in silicon heterojunction solar cells with molybdenum oxide as a hole transport layer," Solar Energy Materials and Solar Cells, vol. 185, pp. 61-65, 2018.

[191] Y. Liu, G. Cen, G. Wang, J. Huang, S. Zhou, J. Zheng, Y. Fu, C. Zhao, and W. Mai, “High performance $\mathrm{MoO} 3-\mathrm{x} / \mathrm{Si}$ heterojunction photodetectors with nanoporous pyramid $\mathrm{Si}$ arrays for visible light communication application," Journal of Materials Chemistry C, vol. 7, no. 4, pp. 917-925, 2019.

[192] Y. Lan, C. Hu, H. Deng, L. Sun, S. Yuan, J. Wang, X. Yu, X. Yang, Y. Zhou, H. Song et al., “MoOx/Au Schottky-Gated Field-Effect Transistors and Their Fast Inverters," Advanced Electronic Materials, p. 1900086, 2019.

[193] I. Irfan and Y. Gao, "Effects of exposure and air annealing on MoOx thin films," Journal of Photonics for Energy, vol. 2, no. 1, p. 021213, 2012. 
[194] I. Irfan, A. James Turinske, Z. Bao, and Y. Gao, "Work function recovery of air exposed molybdenum oxide thin films," Applied Physics Letters, vol. 101, no. 9, p. 093305, 2012.

[195] S. Essig, J. Dréon, E. Rucavado, M. Mews, T. Koida, M. Boccard, J. Werner, J. Geissbühler, P. Löper, M. Morales-Masis et al., "Toward Annealing-Stable Molybdenum-Oxide-Based Hole-Selective Contacts For Silicon Photovoltaics," Solar RRL, vol. 2, no. 4, p. 1700227, 2018.

[196] G. Gregory, M. Wilson, H. Ali, and K. O. Davis, “Thermally Stable Molybdenum Oxide Hole-Selective Contacts Deposited using Spatial Atomic Layer Deposition," in 2018 IEEE 7th World Conference on Photovoltaic Energy Conversion (WCPEC), 2018, pp. 2006-2009.

[197] V. Travkin, A. Y. Luk'yanov, M. Drozdov, E. Vopilkin, P. Yunin, and G. Pakhomov, “Ultrathin metallic interlayers in vacuum deposited $\mathrm{MoOx} /$ metal/MoOx electrodes for organic solar cells," Applied Surface Science, vol. 390, pp. 703-709, 2016.

[198] G. Roelkens, L. Liu, D. Liang, R. Jones, A. Fang, B. Koch, and J. Bowers, "III-V/silicon photonics for on-chip and intra-chip optical interconnects," Laser \& Photonics Reviews, vol. 4, no. 6, pp. 751-779, 2010.

[199] D. Thomson, A. Zilkie, J. E. Bowers, T. Komljenovic, G. T. Reed, L. Vivien, D. Marris-Morini, E. Cassan, L. Virot, J.-M. Fédéli et al., "Roadmap on silicon photonics," Journal of Optics, vol. 18, no. 7, p. 073003, 2016.

[200] G. Roelkens, A. Abassi, P. Cardile, U. Dave, A. De Groote, Y. De Koninck, S. Dhoore, X. Fu, A. Gassenq, N. Hattasan et al., "III-V-on-silicon photonic devices for optical communication and sensing," in Photonics, vol. 2, no. 3. Multidisciplinary Digital Publishing Institute, 2015, pp. 969-1004.

[201] T. Hoang, P. LeMinh, J. Holleman, and J. Schmitz, "Strong efficiency improvement of SOI-LEDs through carrier confinement," IEEE Electron Device Letters, vol. 28, no. 5, pp. 383-385, 2007.

[202] Z. Zhu, H. Zhu, M. Xu, J. Zhong, C. Zhao, D. Chen, and T. Ye, “A novel fin electron-hole bilayer tunnel field-effect transistor," IEEE Transactions on Nanotechnology, vol. 13, no. 6, pp. 1133-1137, Nov 2014

[203] J. Padilla, C. Medina-Bailon, C. Alper, F. Gamiz, and A. Ionescu, “Confinement-induced InAs/GaSb heterojunction electron-hole bilayer tunneling field-effect transistor," Applied Physics Letters, vol. 112, no. 18, p. 182101, 2018.

[204] N. Rodriguez, F. Gamiz, and S. Cristoloveanu, "A-RAM memory cell: Concept and operation," IEEE Electron Device Letters, vol. 31, no. 9, pp. 972-974, 2010.

[205] S. Krishnamurthy and M. Berding, "Full-band-structure calculation of Shockley-Read-Hall recombination rates in InAs," Journal of Applied Physics, vol. 90, no. 2, pp. 848-851, 2001.

[206] N. Li, E. S. Harmon, J. Hyland, D. B. Salzman, T. Ma, Y. Xuan, and P. Ye, “Properties of InAs metaloxide-semiconductor structures with atomic-layer-deposited A12O3 Dielectric," Applied Physics Letters, vol. 92, no. 14, p. 143507, 2008.

[207] Y.-C. Wu, E. Y. Chang, Y.-C. Lin, C.-C. Kei, M. K. Hudait, M. Radosavljevic et al., "Study of the inversion behaviors of Al2O3/InxGa1-xAs metal-oxide-semiconductor capacitors with different In contents," Solid-State Electronics, vol. 54, no. 1, pp. 37-41, 2010.

[208] H. Ko, K. Takei, R. Kapadia, S. Chuang, H. Fang, P. W. Leu et al., “Ultrathin compound semiconductor on insulator layers for high-performance nanoscale transistors," Nature, vol. 468, no. 7321, p. 286, 2010.

[209] K. Takei, S. Chuang, H. Fang, R. Kapadia, C.-H. Liu, J. Nah et al., “Benchmarking the performance of ultrathin body InAs-on-insulator transistors as a function of body thickness," Applied Physics Letters, vol. 99, no. 10, p. 103507, 2011.

[210] N. D. Arora, J. R. Hauser, and D. J. Roulston, "Electron and hole mobilities in silicon as a function of concentration and temperature," IEEE Transactions on Electron Devices, vol. 29, no. 2, pp. 292-295, 1982

[211] C. Canali, G. Majni, R. Minder, and G. Ottaviani, "Electron and hole drift velocity measurements in silicon and their empirical relation to electric field and temperature," IEEE Transactions on Electron Devices, vol. 22, no. 11, pp. 1045-1047, 1975.

[212] M. Ancona and G. Iafrate, "Quantum correction to the equation of state of an electron gas in a semiconductor," Physical Review B, vol. 39, no. 13, p. 9536, 1989

[213] S. Eminente, S. Cristoloveanu, R. Clerc, A. Ohata, and G. Ghibaudo, “Ultra-thin fully-depleted SOI MOSFETs: Special charge properties and coupling effects," Solid-State Electronics, vol. 51, no. 2, pp. 239-244, 2007.

[214] C. Navarro, M. Bawedin, F. Andrieu, B. Sagnes, F. Martinez, and S. Cristoloveanu, "Supercoupling effect in short-channel ultrathin fully depleted silicon-on-insulator transistors," Journal of Applied Physics, vol. 118, no. 18, p. 184504, 2015.

[215] D. Wheeler, A. Seabaugh, L. Froberg, C. Thelander, and L.-E. Wernersson, "Electrical properties of HfO2/InAs MOS capacitors," in Proc. Semiconductor Device Research Symposium. IEEE, 2007, pp. 1-2.

[216] S. Karpov, "ABC-model for interpretation of internal quantum efficiency and its droop in III-nitride LEDs: a review," Optical and Quantum Electronics, vol. 47, no. 6, pp. 1293-1303, 2015.

[217] J. M. Rabaey, A. P. Chandrakasan, and B. Nikolic, Digital integrated circuits. Prentice hall Englewood Cliffs, 2002, vol. 2. 
[218] B. Matveev, M. Aidaraliev, G. Gavrilov, N. Zotova, S. Karandashov, G. Sotnikova, N. Stus, G. Talalakin, N. Il'inskaya, and S. Aleksandrov, "Room temperature InAs photodiode-InGaAs LED pairs for methane detection in the mid-IR," Sensors and Actuators B: Chemical, vol. 51, no. 1-3, pp. 233-237, 1998.

[219] A. Krier, Mid-infrared semiconductor optoelectronics. Springer, 2007, vol. 118.

[220] L. Olsson, C. Andersson, M. Håkansson, J. Kanski, L. Ilver, and U. O. Karlsson, “Charge accumulation at InAs surfaces," Physical Review Letters, vol. 76, no. 19, p. 3626, 1996.

[221] W. Gerlach, H. Schlangenotto, and H. Maeder, "On the radiative recombination rate in silicon," Physica Status Solidi (a), vol. 13, no. 1, pp. 277-283, 1972.

[222] V. Puliyankot, G. Piccolo, R. J. E. Hueting, and J. Schmitz, “Toward GHz Switching in SOI Light Emitting Diodes," IEEE Transactions on Electron Devices, no. 99, pp. 1-8, 2018.

[223] S. Banerjee, "From radical-enhanced to pure thermal ALD of gallium and aluminum nitrides," Ph.D. dissertation, University of Twente, 2019.

[224] G. Yu, G. Wang, H. Ishikawa, M. Umeno, T. Soga, T. Egawa, J. Watanabe, and T. Jimbo, “Optical properties of wurtzite structure $\mathrm{GaN}$ on sapphire around fundamental absorption edge $(0.78-4.77 \mathrm{eV})$ by spectroscopic ellipsometry and the optical transmission method," Applied Physics Letters, vol. 70, no. 24, pp. 3209-3211, 1997.

[225] V. Bermudez, "Study of oxygen chemisorption on the GaN (0001)- $(1 \times 1)$ surface," Journal of Applied Physics, vol. 80, no. 2, pp. 1190-1200, 1996.

[226] C. Wu and A. Kahn, "Investigation of the chemistry and electronic properties of metal/gallium nitride interfaces," Journal of Vacuum Science \& Technology B: Microelectronics and Nanometer Structures Processing, Measurement, and Phenomena, vol. 16, no. 4, pp. 2218-2223, 1998.

[227] A. Rose, "Space-charge-limited currents in solids," Physical Review, vol. 97, no. 6, p. 1538, 1955.

[228] M. A. Lampert and P. Mark, “Current injection in solids," Academic Press Inc., New York, p. 3, 1970.

[229] X. Shen, D. Zhao, Z. Liu, Z. Hu, H. Yang, and J. Liang, “Space-charge-limited currents in GaN Schottky diodes," Solid-State Electronics, vol. 49, no. 5, pp. 847-852, 2005.

[230] H. Hall, M. Awaah, and K. Das, "Deep-level dominated rectifying contacts for n-type GaN films," Physics Status Solidi (a), vol. 201, no. 3, pp. 522-528, 2004.

[231] A. A. Talin, F. Léonard, B. Swartzentruber, X. Wang, and S. D. Hersee, “Unusually strong space-chargelimited current in thin wires," Physical Review Letters, vol. 101, no. 7, p. 076802, 2008.

[232] M. Kumar, T. N. Bhat, M. K. Rajpalke, B. Roul, N. Sinha, A. Kalghatgi, and S. Krupanidhi, “Negative differential capacitance in n-GaN/p-Si heterojunctions," Solid State Communications, vol. 151, no. 5, pp. 356-359, 2011.

[233] M. Kumar, B. Roul, T. N. Bhat, M. K. Rajpalke, A. Kalghatgi, and S. Krupanidhi, “Carrier-transport studies of III-nitride/Si3N4/Si isotype heterojunctions," Physica Status Solidi (a), vol. 209, no. 5, pp. 994-997, 2012.

[234] K. Saron, M. Hashim, N. Naderi, and N. K. Allam, “Interface properties determined the performance of thermally grown GaN/Si heterojunction solar cells," Solar Energy, vol. 98, pp. 485-491, 2013.

[235] R. Ghosh and D. Basak, "Electrical and ultraviolet photoresponse properties of quasialigned $\mathrm{ZnO}$ nanowires/p-Si heterojunction," Applied Physics Letters, vol. 90, no. 24, p. 243106, 2007.

[236] J. Ye, S. Gu, S. Zhu, W. Liu, S. Liu, R. Zhang, Y. Shi, and Y. Zheng, “Electroluminescent and transport mechanisms of n-ZnO/ p-Si heterojunctions," Applied Physics Letters, vol. 88, no. 18, p. 182112, 2006.

[237] Y. I. Alivov, J. Van Nostrand, D. C. Look, M. Chukichev, and B. Ataev, “Observation of $430 \mathrm{~nm}$ electroluminescence from $\mathrm{ZnO} / \mathrm{GaN}$ heterojunction light-emitting diodes," Applied Physics Letters, vol. 83, no. 14, pp. 2943-2945, 2003.

[238] F.-C. Chiu, H.-W. Chou, and J. Y.-m. Lee, “Electrical conduction mechanisms of metal/La2O3/Si structure," Journal of Applied Physics, vol. 97, no. 10, p. 103503, 2005.

[239] M. E. Levinshtein, S. L. Rumyantsev, and M. S. Shur, Properties of Advanced Semiconductor Materials: GaN, AIN, InN, BN, SiC, SiGe. John Wiley \& Sons, 2001.

[240] G. Roberts and F. W. Schmidlin, "Study of localized levels in semi-insulators by combined measurements of thermally activated ohmic and space-charge-limited conduction," Physical Review, vol. 180, no. 3, p. 785, 1969.

[241] J. Fedison, T. Chow, H. Lu, and I. Bhat, "Electrical characteristics of magnesium-doped gallium nitride junction diodes," Applied Physics Letters, vol. 72, no. 22, pp. 2841-2843, 1998.

[242] E. Suzuki, D. K. Schroder, and Y. Hayashi, "Carrier conduction in ultrathin nitrided oxide films," Journal of Applied Physics, vol. 60, no. 10, pp. 3616-3621, 1986.

[243] M. P. Houng, Y. H. Wang, and W. J. Chang, “Current transport mechanism in trapped oxides: A generalized trap-assisted tunneling model," Journal of Applied Physics, vol. 86, no. 3, pp. 1488-1491, 1999.

[244] O. Blank, H. Reisinger, R. Stengl, M. Gutsche, F. Wiest, V. Capodieci, J. Schulze, and I. Eisele, “A model 
for multistep trap-assisted tunneling in thin high-k dielectrics," Journal of Applied Physics, vol. 97, no. 4, p. $044107,2005$.

[245] O. Mitrofanov and M. Manfra, "Poole-Frenkel electron emission from the traps in AlGaN/GaN transistors," Journal of Applied Physics, vol. 95, no. 11, pp. 6414-6419, 2004.

[246] E. Arslan, S. Bütün, and E. Ozbay, "Leakage current by Frenkel-Poole emission in Ni/Au Schottky contacts on Al 0.83 In 0.17 N/AlN/GaN heterostructures," Applied Physics Letters, vol. 94, no. 14, p. 142106, 2009.

[247] A. Fiory and N. Ravindra, "Light emission from silicon: Some perspectives and applications," Journal of Electronic Materials, vol. 32, no. 10, pp. 1043-1051, 2003

[248] T.-H. Cheng, Y. Chu-Su, C.-S. Liu, and C.-W. Lin, "Phonon-assisted transient electroluminescence in Si," Applied Physics Letters, vol. 104, no. 26, p. 261102, 2014.

[249] S. Dutta, V. Agarwal, R. J. E. Hueting, J. Schmitz, and A.-J. Annema, “Monolithic optical link in siliconon-insulator CMOS technology," Optics Express, vol. 25, no. 5, pp. 5440-5456, 2017.

[250] S. Dutta, G. J. Wienk, R. J. E. Hueting, J. Schmitz, and A.-J. Annema, “Optical Power Efficiency Versus Breakdown Voltage of Avalanche-Mode Silicon LEDs in CMOS," IEEE Electron Device Letters, vol. 38, no. 7, pp. 898-901, 2017.

[251] G. Qin, Y. Huang, J. Lin, L. Zhang, B. Zong, and B. Zhang, “Electroluminescence from Au/native oxide/p-Si and its correlation to that from $\mathrm{Au}$ /porous $\mathrm{Si}$," Solid State Communications, vol. 94, no. 8, pp. 607-612, 1995.

[252] H. Kroemer, "Two integral relations pertaining to the electron transport through a bipolar transistor with a nonuniform energy gap in the base region," Solid-State Electronics, vol. 28, no. 11, pp. 1101-1103, 1985.

[253] J. Wu, W. Walukiewicz, K. Yu, W. Shan, J. Ager Iii, E. Haller, H. Lu, W. J. Schaff, W. Metzger, and S. Kurtz, "Superior radiation resistance of In 1-x Ga x N alloys: full-solar-spectrum photovoltaic material system," Journal of Applied Physics, vol. 94, no. 10, pp. 6477-6482, 2003.

[254] Y. Tang, Z. Chen, H. Song, C. Lee, H. Cong, H. Cheng, W. Zhang, I. Bello, and S. Lee, “Vertically aligned p-type single-crystalline GaN nanorod arrays on n-type $\mathrm{Si}$ for heterojunction photovoltaic cells," Nano Letters, vol. 8, no. 12, pp. 4191-4195, 2008.

[255] P. Favennec, H. L'haridon, M. Salvi, D. Moutonnet, and Y. Le Guillou, “Luminescence of erbium implanted in various semiconductors: IV, III-V and II-VI materials," Electronics Letters, vol. 25, no. 11, pp 718-719, 1989.

[256] M. A. Lourenço, M. A. Hughes, K. T. Lai, I. M. Sofi, W. Ludurczak, L. Wong, R. M. Gwilliam, and K. P. Homewood, "Silicon-Modified Rare-Earth Transitions-A New Route to Near-and Mid-IR Photonics," Advanced Functional Materials, vol. 26, no. 12, pp. 1986-1994, 2016.

[257] A. Steckl, M. Garter, R. Birkhahn, and J. Scofield, “Green electroluminescence from Er-doped GaN Schottky barrier diodes," Applied Physics Letters, vol. 73, no. 17, pp. 2450-2452, 1998.

[258] J. B. You, X. W. Zhang, S. G. Zhang, J. X. Wang, Z. G. Yin, H. R. Tan, W. J. Zhang, P. K. Chu, B. Cui, A. M. Wowchak, A. M. Dabiran, and P. P. Chow, "Improved electroluminescence from n-ZnO/AlN/p-GaN heterojunction light-emitting diodes," Applied Physics Letters, vol. 96, no. 20, p. 201102, 2010.

[259] C. B. Han, C. He, and X. J. Li, "Near-Infrared Light Emission from a GaN/Si Nanoheterostructure Array," Advanced Materials, vol. 23, no. 41, pp. 4811-4814, 2011.

[260] C. B. Han, C. He, X. B. Meng, Y. R. Wan, Y. T. Tian, Y. J. Zhang, and X. J. Li, “Effect of annealing treatment on electroluminescence from GaN/Si nanoheterostructure array," Optics Express, vol. 20, no. 5, pp. 5636-5643, 2012.

[261] S. Banerjee, A. A. Aarnink, D. J. Gravesteijn, and A. Y. Kovalgin, "Thermal Atomic Layer Deposition of Polycrystalline Gallium Nitride," The Journal of Physical Chemistry C, vol. 123, no. 37, pp. 23 214-23 225, 2019.

[262] D. K. Schroder, Semiconductor material and device characterization. John Wiley \& Sons, 2006.

[263] F. Werner, "Hall measurements on low-mobility thin films," Journal of Applied Physics, vol. 122, no. 13, p. 135306, 2017.

[264] T. Ando, A. B. Fowler, and F. Stern, "Electronic properties of two-dimensional systems," Reviews of Modern Physics, vol. 54, no. 2, p. 437, 1982.

[265] A. Hartstein and A. Fowler, "High temperature 'variable range hopping' conductivity in silicon inversion layers," Journal of Physics C: Solid State Physics, vol. 8, no. 11, p. L249, 1975.

[266] J. Garrido, T. Heimbeck, and M. Stutzmann, “Temperature-dependent transport properties of hydrogen-induced diamond surface conductive channels," Physical Review B, vol. 71, no. 24, p. 245310, 2005.

[267] N. F. Mott, M. Pepper, S. Pollitt, R. Wallis, and C. Adkins, "The anderson transition," Proceedings of the Royal Society of London. A. Mathematical and Physical Sciences, vol. 345, no. 1641, pp. 169-205, 1975.

[268] E. Arnold, "Conduction mechanisms in bandtails at the Si-SiO2 interface," Surface Science, vol. 58, no. 1, 
pp. 60-70, 1976.

[269] M. Salis, A. Anedda, F. Quarati, A. Blue, and W. Cunningham, "Photocurrent in epitaxial GaN," Journal of Applied Physics, vol. 97, no. 3, p. 033709, 2005.

[270] T. Zhu and R. A. Oliver, “Unintentional doping in GaN," Physical Chemistry Chemical Physics, vol. 14, no. 27, pp. 9558-9573, 2012.

[271] R. Chen, W. Zhou, and H. Sing Kwok, “Top-gate thin-film transistors based on GaN channel layer," Applied Physics Letters, vol. 100, no. 2, p. 022111, 2012.

[272] R. Chen, W. Zhou, M. Zhang, and H. S. Kwok, "Top-gate GaN thin-film transistors based on AlN/GaN heterostructures," IEEE Electron Device Letters, vol. 33, no. 9, pp. 1282-1284, 2012.

[273] _ _Bottom-gate thin-film transistors based on GaN active channel layer," IEEE Electron Device Letters, vol. 34, no. 4, pp. 517-519, 2013.

[274] H. Umezawa, H. Taniuchi, T. Arima, M. Tachiki, and H. Kawarada, "Potential applications of surface channel diamond field-effect transistors," Diamond and Related Materials, vol. 10, no. 9-10, pp. 17431748, 2001

[275] G. Gupta and R. J. E. Hueting, “Characteristic C-V profile as a signature for electrostatic doping in FDSOI," in 2020 Joint International EUROSOI Workshop and International Conference on Ultimate Integration on Silicon (EUROSOI-ULIS). IEEE, 2020, (accepted).

[276] V. P. Trivedi and J. G. Fossum, "Quantum-mechanical effects on the threshold voltage of undoped double-gate MOSFETs," IEEE Electron Device Letters, vol. 26, no. 8, pp. 579-582, Aug 2005.

[277] S. Evseev, L. Nanver, and S. Milosavljevic, "Ring-gate MOSFET test structures for measuring surfacecharge-layer sheet resistance on high-resistivity-silicon substrates," in 2006 IEEE International Conference on Microelectronic Test Structures, 2006, pp. 3-8.

[278] L. Nanver, X. Liu, and T. Knezevic, "Test structures without metal contacts for DC measurement of 2Dmaterials deposited on silicon," in 2018 IEEE International Conference on Microelectronic Test Structures (ICMTS), 2018, pp. 69-74. 


\section{LIST OF PUBLICATIONS}

\section{Journal articles}

1. G. Gupta, D.T. Shivakumar, L.K. Nanver and R.J.E. Hueting, "Investigation of $\mathrm{Pd} / \mathrm{MoO}_{\mathrm{x}} / \mathrm{n}$-Si diodes for bipolar transistor and light-emitting device applications", (submitted to Journal of Applied Physics)

2. G. Gupta, F. Mema and R.J.E. Hueting, "Electron-hole bilayer light-emitting device: Concept and operation", Solid-State Electronics, vol. 168, 107726, June, 2020.

3. G. Gupta, S. Banerjee, S. Dutta, A.A.I. Aarnink, J. Schmitz, A.Y. Kovalgin and R.J.E. Hueting, "Charge carrier transport and electroluminescence in atomic layer deposited poly-GaN/c-Si heterojunction diodes", Journal of Applied Physics, vol. 124, no. 8, August, 2018.

4. G. Gupta, S. Dutta, S. Banerjee and R.J.E. Hueting, "Minority carrier injection in high-barrier Si Schottky diodes", IEEE Transactions on Electron Devices, vol. 65, no. 4, April, 2018.

5. G. Gupta, B. Rajasekharan and R.J.E. Hueting, "Electrostatic doping in semiconductor devices", IEEE Transactions on Electron Devices, vol. 64, no. 8, August, 2017.

\section{Conference proceedings}

1. G. Gupta and R.J.E. Hueting, "Characteristic $C-V$ profile as a signature for electrostatic doping in FD-SOI", Joint International EUROSOI Workshop and International Conference on Ultimate Integration on Silicon (EUROSOIULIS), 2020, Caen, Normandy, France (accepted).

2. G. Gupta, F. Mema and R.J.E. Hueting, "InAs Electron-Hole Bilayer LED", Joint International EUROSOI Workshop and International Conference on Ultimate Integration on Silicon (EUROSOI-ULIS), 2019, Grenoble, France.

3. G. Gupta, D.T. Shivakumar, R.J.E. Hueting and L.K. Nanver, "In search of a hole inversion layer in $\mathrm{Pd} / \mathrm{MoOx} / \mathrm{Si}$ diodes through $\mathrm{I}-\mathrm{V}$ characterization using dedicated ring-shaped test structures", International Conference on Microelectronic Test Structures (ICMTS), 2019, Kita Kyushu, Japan.

\section{Book chapter}

1. R.J.E. Hueting and G. Gupta, "Electrostatic Doping and Devices", Springer Handbook of Semiconductor Devices (under-revision). 


\section{Others}

1. G. Gupta, F. Mema and R.J.E. Hueting, "InAs Electron-Hole Bilayer LED", oral presentation at SAFE 2019 Workshop, Delft.

2. G. Gupta and R.J.E. Hueting, "Polycrystalline GaN: Material and Devices", oral presentation at the MESA+ Symposium 2018, Enschede.

3. G. Gupta and R.J.E. Hueting, "Electrostatic Doping in Dimensionally-Scaled Semiconductor Devices", poster presentation at the SAFE 2018 Workshop, Enschede. (Best poster award)

4. G. Gupta and R.J.E. Hueting, "Electrostatic Doping in Semiconductor Devices", poster presentation at the MESA+ Symposium 2017, Enschede. 


\section{ACKNOWLEDGEMENTS}

Finally, it's time to extend my gratitude and say "thank-you" from the bottom of my heart to all those wonderful people who helped me in shaping my PhD journey in the last four years and without whose support this thesis would not have existed as of today.

I would like to start by thanking my $\mathrm{PhD}$ committee members for taking their time out reading this thesis and for their valuable input. My very special thanks to my daily supervisor, Dr. Ray Hueting who guided me at every single step of this journey both at professional and personnel level. Thanks Ray for all your time and attention you showered on me during these four years. There has not been a single day except weekends (ok including weekends) when I have not bothered you with mail, presentation, paper, thesis etc and you have always reverted back with your lighting fast response. I can say this without an iota of doubt that you were the best supervisor I could ever ask for. You pretty much define the very existence of this thesis, so thank you so much. I would also like to sincerely thank Prof. Lis Nanver, first and foremost for accepting to be my promotor. I believe its a lifelong privilege for me to call myself your student. Thanks for all your guidance and support throughout this work without which it would have been difficult to explore, in particular, the experimental side of this work. Under your supervision, this work has been a very fulfilling experience for me where I learned and unlearned several aspects of the device physics and technology. You gave me the opportunity to experience the thrill of electrical measurements. Your love and passion for the subject is infectious and is something that I will carry with me in my journey ahead. So thank you for everything.

My sincere gratitude to Prof. Jurriaan Schmitz for all your technical as well as administrative support throughout my term and for being my promotor. Thanks for all your valuable time spent in critically reading and reviewing all my manuscripts and for giving much needed direction and focus to all my papers. Without your strict internal review process which I do admit gave me some hard time, I couldn't have experienced the early success in the external journal reviews. At this juncture, I would also like to thank Dr. Alexey Kovalgin for bringing this exciting polyGaN project to Twente and selecting me for the PhD position. Thanks for giving me all the freedom to explore and experience different research directions in this project and also for all your inputs and feedback during our meetings. Also my sincere thanks to other IDS group members: Dirk, Cora and Rob for always being approachable for any help or discussion and for all your interesting feedback on my work during our group meetings.

Thanks Sanders for all your initial help in the measurement lab, TCAD and Cadence set-up which gave me a smooth start in my work, also for proof-reading my first paper and specially for introducing me to the staff badminton activity. Those lunch time sessions really helped me in relieving my work stress and made me more productive. Thanks Tom for being there and for making me feel that 
you will always be there to help when needed. Specially thanks for your help in dicing the difficult sapphire samples without which I couldn't have done my Hall measurements. Thanks Gerard and Henk from ICD group for all your help in measurement lab and also for your support in TCAD tools. Thanks Remke for actually starting this whole story, from introducing me to the vacancy, arranging interviews and for working out all the nitty-gritty of the process as to make my transition from India to NL and my stay thereafter smooth and hassle-free. Also thanks Marthe and Susan for all your timely help and support in administration matters during last two years of my PhD.

I would also like to thank my fellow PhD colleagues for all their help in work as well as personnel matters and for making this journey overall fun-filled and exciting learning experience. Thanks Satadal, for being there all the time (even after moving to Delft), for co-authoring the papers, for proof-reading my work, for all our stimulating discussions and your critical feedback and for all your help in TCAD, experimental as well as personnel matters for which I can't thank you enough. I cherished our (not-so) random friday evening experiments in measurement lab which actually helped me realizing that research is indeed fun (sometime!). Thanks Sourish for being my other-half in the poly-GaN project, I think we were a good team and our project was a success. Thanks for all those samples you prepared for me and for discussion on and off the topic. Also thanks for all those bike trips I joined with you in my first year and for showing me the beautiful country side. Thanks Shiva, my friend and project partner in other half of my work. Without your help and collaboration my electrostatic doping ideas would not have come out of TCAD. Thanks for believing in my ideas and for all your experimental help and for all those innumerable samples you made for me. Together we have witnessed the ups and down of this work. Also thanks for all those interesting discussions we had on Indian politics and global affairs time to time.

Thanks Thiomir for your all help in TCAD and measurements in particular and device physics in general. I benefited a lot from your device experience, all those small tricks and codes you provided proved to be a great help and time saver for me. After Satadal, you took over as a person whom I can rely on and bother on day-to-day basis (ok hourly!) for any small or big issues (un)related to work. Thanks Maurits for being there, for being my dutch guide time to time and for your help in day-to-day matters. I missed showing you India around during your trip but may be next time (but avoid peak Indian summer time). Thanks Arnoud for being a wonderful officemate, in-house chemist and of-course a trusted friend. Thanks for all your support and help in my research work as well as on general issues related to dutch life. I cherished our fun moments during group outings and Christmas dinners. Thanks Oguzhan and Xingyu for being there, without you guys being around, office would not have been that fun and also thanks for your help in various matters time to time. Thanks Svetlana for all help during my initial days in office, for showing me around and getting me accustomed to the new environment. Also thanks to Jaihui and Mengdi for that matter. Special thanks to all those colleagues who have spent their valuable $\mathrm{PhD}$ time in preparing samples for me: Bernhard, Oguzhan and Xingyu, believe me it was a great help in my research and I can't acknowledge you enough. Thanks Kees for being a nice and friendly colleague around, our Japan trip is a very special part of my PhD journey, thanks for making it fun and memorable. Thanks Ran for adding the much needed light-hearted first-year PhD charm to our over-boarded final year 
PhD room. Also thanks for all those delicious cakes you prepared for us. Thanks Kevin for your help in AFM measurements. Thanks Max for being around and for your all help and support in the measurement lab. Special thanks to people outside IDS group, Yigitcan (NE) and Yura (IMS) for all your technical help and support in doing Hall measurements on my samples. Thanks to my other Indian colleagues around: Vijay, Anoop and Harijot with whom I shared some light moments in and around office corridors. Also thanks to MESA+ Batavierenrace team for providing me the wonderful opportunity to be part of this event for two consecutive years which I throughly enjoyed. Running around the beautiful country side with my "fiets" (bike) partner at odd hours in the morning was indeed an unforgettable experience. Finally, special thanks to Maurits and Ran for being my paranymphs, it means a lot to me.

It is also important here to acknowledge my friends outside work for making me realize that there is indeed a life beyond PhD research to live, enjoy and cherish. Thanks to all my friends in Calslaan (Nikhil, Ajinkya, Amit, Arshad), snuifstraat (Nishant, Jagadesh), badminton friends (Giri, Anwar), family friends (Vishal, Bina, Atasi and Anoop) with whom I shared some memorable and fun moments. The story here cannot end without mentioning and thanking Sahil for starting this journey together, being there in my ups and downs, for all outings, parties, memorable trips and for adding special "punjabi-tadka" in everything we did together.

I would also like to pay my gratitude to people who actually inspired and motivated to take this journey. Thanks to Prof. Rao (IIT-Bombay), Prof. Ghosh (IISc Bangalore) and Dr. Usikov (USA) for all their help and support in undertaking this journey. Thanks Kamal and Avichal for pushing me out of my comfort zone and taking up the PhD challenge. Thanks Nidhi, Anzar, Rao and Sandeep for being so supportive and helpful whenever needed.

Now it's time to thank to my lovely family: first and foremost to my late father who define my existence, who taught me to work hard and excel in every corner of life, whose innumerable sacrifices made the person I am today. I could not thank enough to my mother for her unconditional love and support always in whatever I do and for giving me the freedom and encouragement to chase my dreams. Thanks to my other family members: my sister, my father- and mother-in-law, Rajendra mama, Nihit, Avish and Kratika for being such a wonderful and supportive family thoughout my journey. Special thanks to my cute little niece Mishu (my bundle of joy!) whose photos and videos makes me smile all the time and help me to regain my lighter and saner side even at difficult times. Last but not the least, a very special thanks to the person who stood with me throughout this journey, who witnessed my highs and lows, supported me, listened to me patiently, laughed on my poor jokes and with whom I everyday celebrate the event called life, my lovely wife Akshita. Thanks Akshita for all your love and support, patience and understanding you showered on me throughout this journey. Thanks for bearing me, I am sorry for not being your good dance student (so far). Without you being around, this journey would not have been so memorable and fulfilling. And yes, I have not forgotten, we will go to Santorini !!

Finally, my very special thanks to Enschede and to dutch people in general who left a very positive influence on me during my stay here and for being a wonderful host. Thanks for making me (as a foreigner) feel welcomed with your open, friendly and helping attitude everywhere and for bearing me with my poor dutch skills. With this, at last, I rest my case here and look forward to my journey ahead !! 